\title{
Exercises in Developing Bebop Scale Language for Collegiate Jazz Students
}

Lars Swanson

West Virginia University, Iwswanson@mix.wvu.edu

Follow this and additional works at: https://researchrepository.wvu.edu/etd

Part of the Music Theory Commons, and the Other Music Commons

\section{Recommended Citation}

Swanson, Lars, "Exercises in Developing Bebop Scale Language for Collegiate Jazz Students" (2020). Graduate Theses, Dissertations, and Problem Reports. 7594.

https://researchrepository.wvu.edu/etd/7594

This Problem/Project Report is protected by copyright and/or related rights. It has been brought to you by the The Research Repository @WVU with permission from the rights-holder(s). You are free to use this Problem/Project Report in any way that is permitted by the copyright and related rights legislation that applies to your use. For other uses you must obtain permission from the rights-holder(s) directly, unless additional rights are indicated by a Creative Commons license in the record and/ or on the work itself. This Problem/Project Report has been accepted for inclusion in WVU Graduate Theses, Dissertations, and Problem Reports collection by an authorized administrator of The Research Repository @ WVU. For more information, please contact researchrepository@mail.wvu.edu. 
Graduate Theses, Dissertations, and Problem Reports

2020

Exercises in Developing Bebop Scale Language for Collegiate Jazz Students

LARS SWANSON

Follow this and additional works at: https://researchrepository.wvu.edu/etd

Part of the Music Theory Commons, and the Other Music Commons 
Exercises in Developing Bebop Scale Language

\author{
Lars Swanson \\ Project submitted \\ to the College of Creative Arts \\ at West Virginia University \\ in partial fulfillment of the requirements for the degree of \\ Master of Music in \\ Jazz Pedagogy.
}

Jared Sims, PhD, Chair

Travis Stimeling, $\mathrm{PhD}$

Andrew Kohn, PhD

Department of Music

Morgantown, West Virginia

2020

Keywords: jazz pedagogy, jazz etudes, bebop scales, college jazz program Copyright 2018 Lars Swanson 


\section{ABSTRACT \\ Exercises in Developing Bebop Scale Language}

\section{Lars Swanson}

In this document, major, dominant and minor bebop scales are introduced and engaged through a series of etudes for $\mathrm{C}$ instruments, Alto Sax, Tenor Sax, Trumpet, Trombone and Bass. The methodology used is derived from a series of private lessons in which bebop scales were introduced and then engaged over chord progressions. Backing tracks are utilized and linked. 
Methods.............................................1

Etudes...............................................5

Link to play along recordings.....................................5

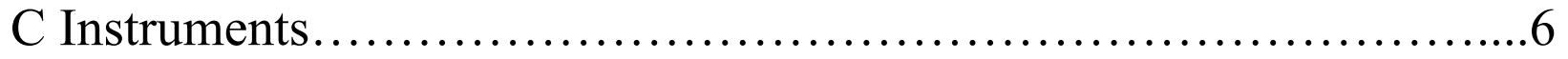

Alto Sax.....................................................27

Trumpet...................................................... 53

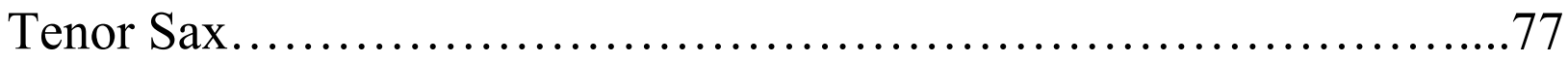

Trombone.......................................................101

Bass ....................................................... 113 
A clear lacking skill set through the majority of the jazz department at WVU is the ability to engage fundamental eighth-note language. Often, beginning jazz students have trouble engaging any combination of phrasing, rhythm, harmony. While many of these issues can be solved by listening, many students lack guidance or discipline to deeply listen to jazz music as often as they should to develop a sound concept on their instrument. Also, often students are enrolled in a combo setting and not enrolled in some kind of improvisation lesson or class. No method can make up for the years of deep listening it takes to develop a mature sound concept as a jazz improviser, especially in the pressure of a weekly hour group lesson. However, etudes and method books can help partially bridge the gaps that would keep a beginning jazz student from achieving informed musical phrasing due to lack of a mature sound concept.

The methods presented in this text are influenced by a series of private lessons given to jazz students at WVU. Through a series of 5-8 lessons, students learned and reviewed by ear the three bebop scales presented in the book. Once these scales were solid, students engaged scalar patterns starting on different scale degrees over the chord progressions of standard jazz tunes. This was done in quarter notes and eighth notes in order to help with rhythm reinforcement, especially for bassists. This method book streamlines that process by working through scales one at a time and then applying these scales directly over chord progressions through a series of etudes.

Scale Exercises 1, 2, and 3 are transcriptions of the aforementioned exercises used with students in private lessons at the beginning of the semester in Fall of 2019. Starting with playing the scale in quarter notes developed a fundamental rhythmic sense for bassists, and in private 
lessons the quarter note section was often vamped until the scale could successfully be played. The student would then slowly transition to 8th note lines, stop playing, and then play the exercise as transcribed in the text, alternating between quarter and eighth notes. Given the focus of the book to transition students to playing fluent eighth note language, the transition to eighth notes is immediate rather than gradual in scale exercises.

The minor bebop scale is the fifth mode of the dominant bebop scale. This scale was included because it illustrates a fundamental motion from predominant to dominant chord, the ii-V progression, and is illustrated thoroughly in this manner in the book.

The first two Etudes in the book, aptly named "Etude 1" and "Etude 2," are derived from the major bebop scale. The melody and chords were originally composed in order to maximize the specific major chord language, as there are not many jazz tunes with static, varied parallel major chords. The second set of etudes, "Etude 3" and "Etude 4" apply the dominant bebop scale in different phrases. The first exercise is a contrafact of the Wayne Shorter tune "Adam's Apple." The second is a dominant-heavy reharmonization of the chord progression to the tune "Scrapple from the Apple." The final etude, "Etude 5" heavily uses the minor bebop scale over the chords to the Ellington tune "Satin Doll."

Exercises are organized differently for different purposes. The first three etudes begin with a scalar exercise section, then present a chorus of notated jazz phrases. The scalar section are often marked "simile" to reinforce phrasing as an aural activity; however, solo sections are heavily notated with phrase markings and articulations. Some of the etudes contain heavily notated scale pattern sections because the patterns are heavily varied rhythmically. 
Different instruments feature different notation in these method books. There are no obvious swing articulation markings in the bass book besides accents because of the inherent nature of bass articulation. The parts transposed for different horns feature more heavy articulations and more space to breathe in scale exercises.

The accompanimental playalong files feature myself on bass, Michael Jones on keyboard and David Hanson on drums. The session was engineered by WVU student Chris Kuskey and we recorded live in the jazz office in the CAC.

The rationale for using bebop scales is rooted in the history of jazz music, taken from added chromatic notes used by bebop musicians. Reportedly first named "bebop scales" by David Baker, these major scales and mixolydian modes use added chromatic notes which place chord tones on the strong beats in four.

Contrafacts have been a standard practice in the history of jazz, and this standard practice has pervaded pedagogical material. Many method books, notably the Greg Fishman Jazz Saxophone Etudes, utilize contrafacts in their compositional material. David Baker's How To Learn Tunes utilizes wittily named contrafacts to explain different methods of tune learning.

These books are meant for beginning jazz students at a beginning collegiate level of technical aptitude on their instrument. Because of this, some of the material is challenging to sight read. Compared to other method books, such as the Greg Fishman etudes or Jim Snidero Jazz Conception books, this method book contains much more meticulous articulations. This higher density of articulation is most readily illustrated in the Jazz Conception for Saxophone series by Lennie Niehaus. 
A play along CD or download is standard practice for most jazz etude books. Recording at multiple tempos was influenced by a vision to distribute the play along for the book: produce an app that had real instruments recorded with the flexibility of an app such as iRealB in tempo and key signature. However, when options were explored this was beyond the financial scope of the project. Multiple tempi help increase the playability and flexibility of these etudes for different skill levels and instruments. The piano and bass are panned separately from each other, a technique used in the Jamey Aebersold play along series. This increases the flexibility of the recordings to be used by bass players, piano players, and horn players. To get rid of bass or piano one can simply remove one headphone or unplug one speaker.

This method book focuses on fundamental issues of harmony, rhythm and phrasing. In the future, an expansion or another book could explain the method of engaging scales specifically over chord progressions using fill-in-the-blank examples or etudes with thorough justification, and by giving suggestions of tunes to apply these scales and patterns over.

The idea of the application that inspired the recording methods of the play along tracks will not be abandoned. However, the financial scope of that project would necessitate a broader audience than people that use this book only. 
Access play along recordings HERE ->

https://drive.google.com/drive/folders/1x7qCtGAp4s5J4II0j7iJ3414fAGyFeTU?usp=sharing 


\section{SCALE EXERCISE 1 - MAJOR BEBOP SCALES}

TRACK $1-100 \mathrm{BPM}$

TRACK 2 - $120 \mathrm{BPM}$

CMAj7

TRACK 3 - 140 BPM

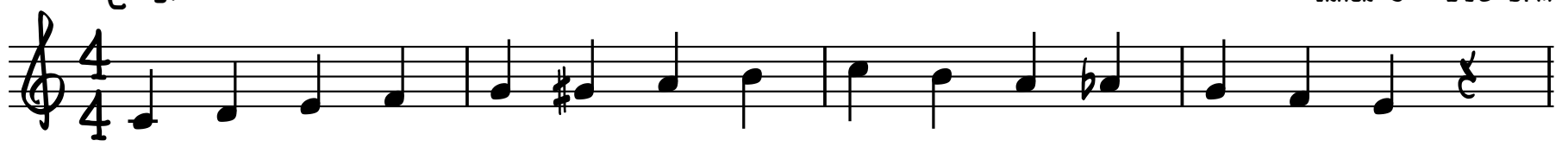

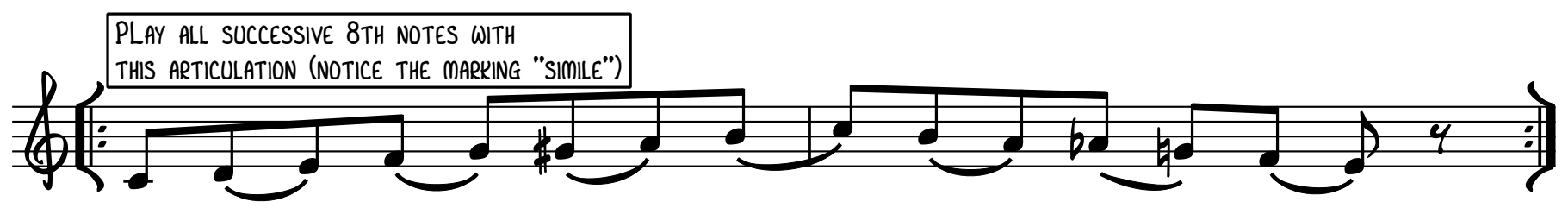

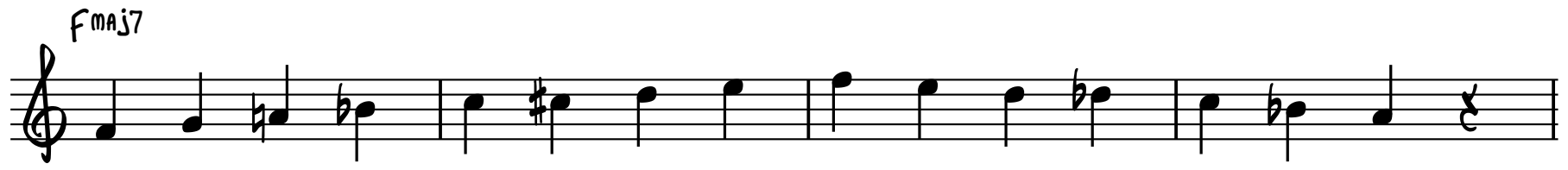

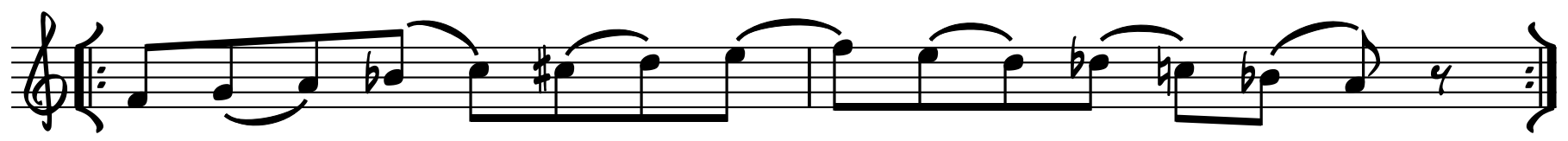

Bbmaj7

$f^{\text {SIMILE }}$

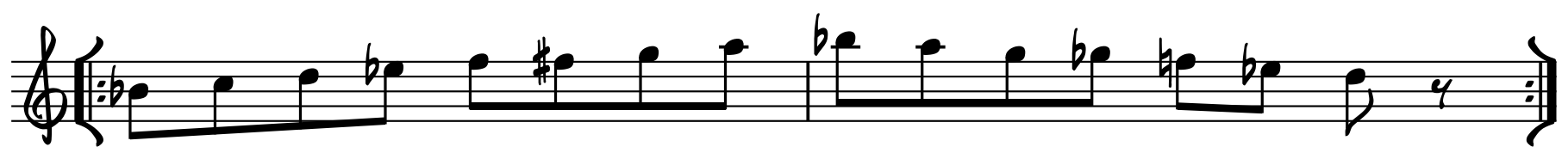

$\int_{b 0}^{E b^{\text {maj7 }}}$

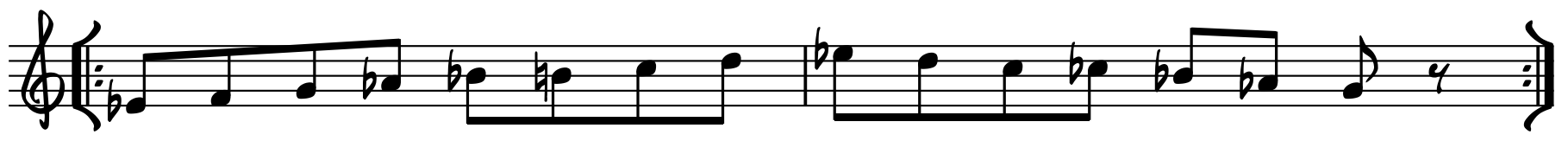

$\int_{b}^{A b^{\text {Aj }} 7}$ 


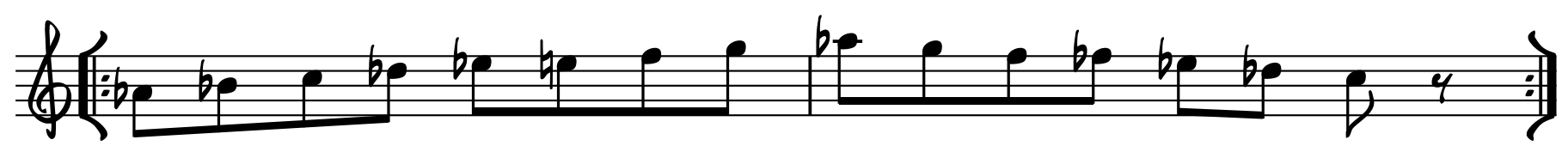
Domisi

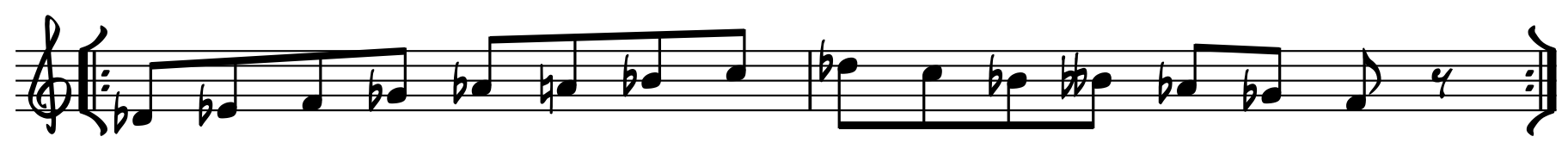
fसmiz 轨(

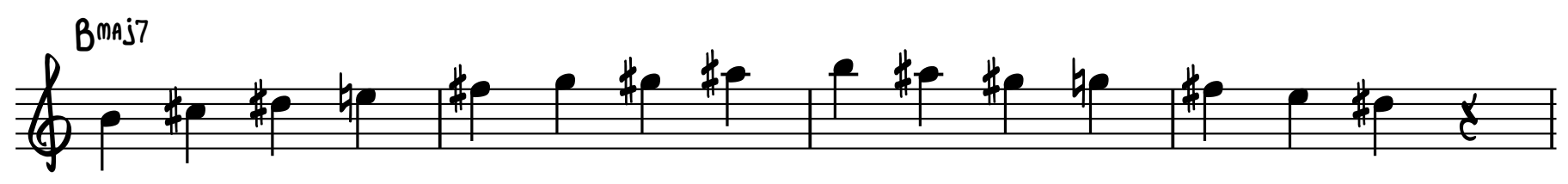

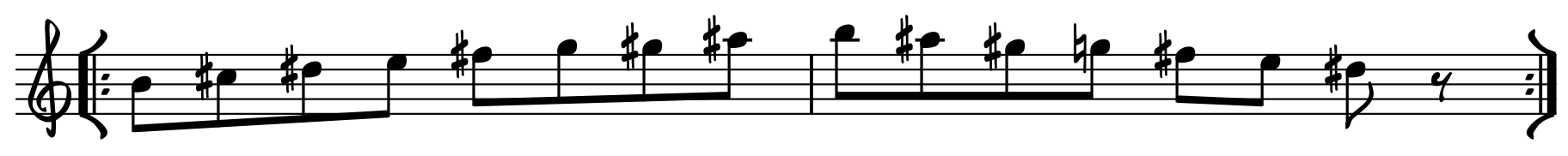
Emancto

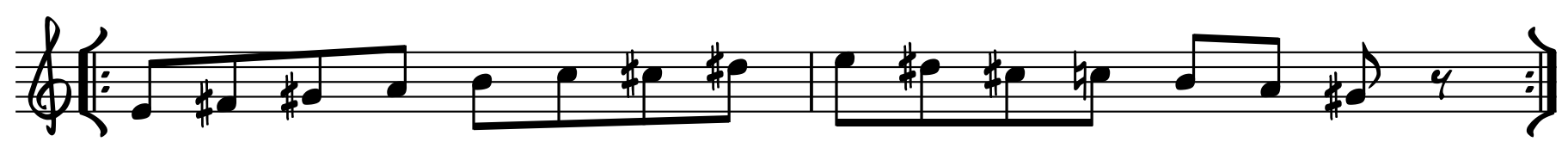
$e^{\text {Amis7 }}$ 

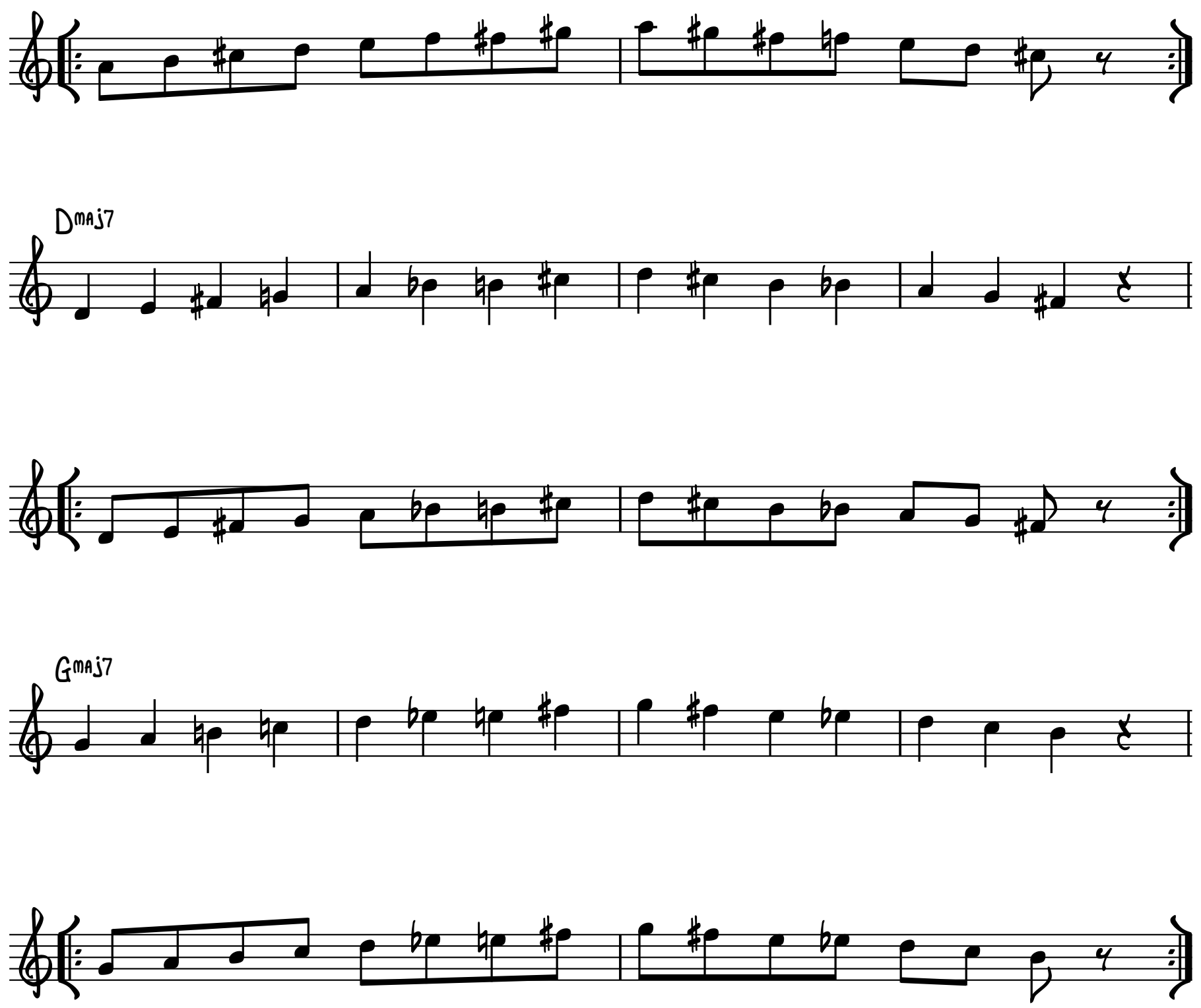
ONCE YOU HAVE MASTERED THIS AT THE FASTEST TEMPO, TRY TO
PRACTICE THESE SCALES STARTING ON THE 3RD, 5TH AND 6TH SCALE DEGREES.

Cmaj7

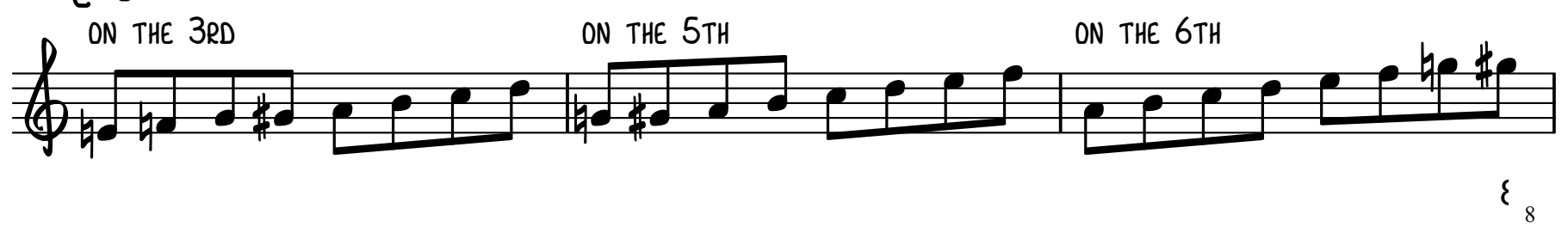




\section{SCALE EXERCISE 2 - DOMINANT BEBOP SCALES}

TRACK 10 - 100 BPM TRACK 11 - 120 BPM

C7 TRACK 12 - 140 BPM
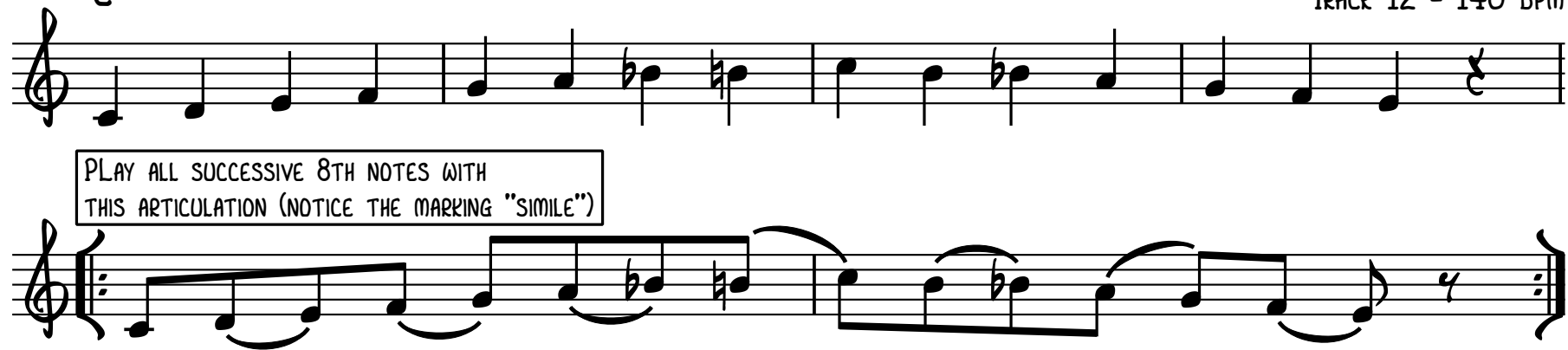

F7

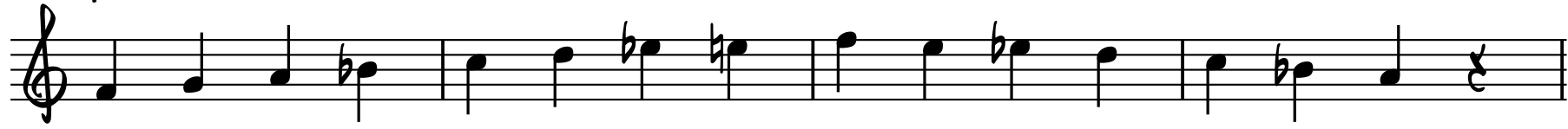

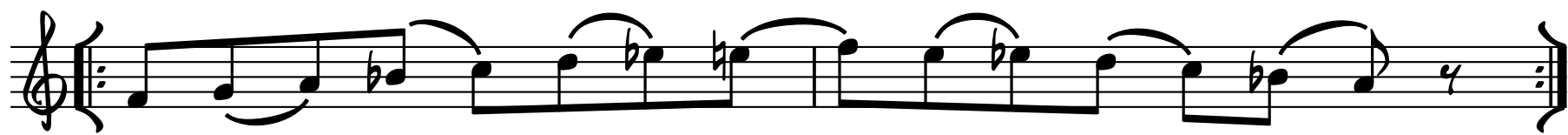

Bb7

$\oiint^{\text {SIMILE }}$

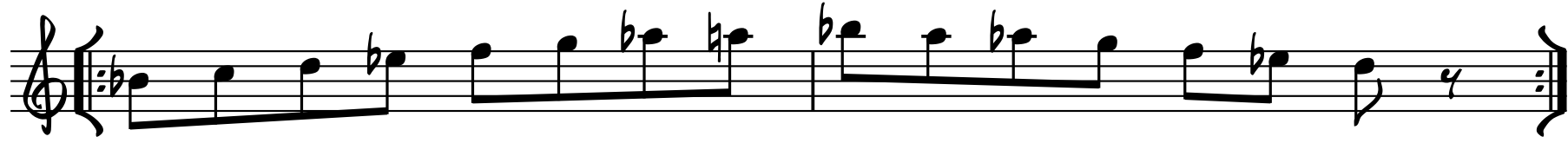

$\oiint_{b a}^{E b 7}$

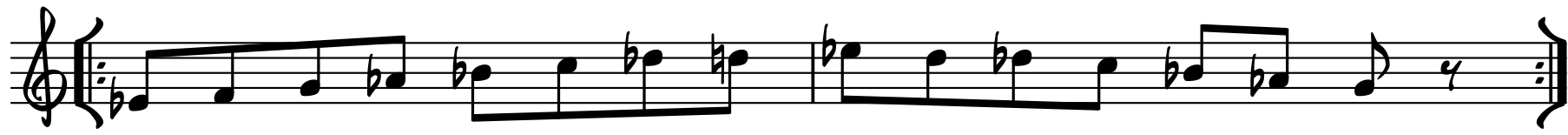

$\int_{b}{ }^{A b 7}$

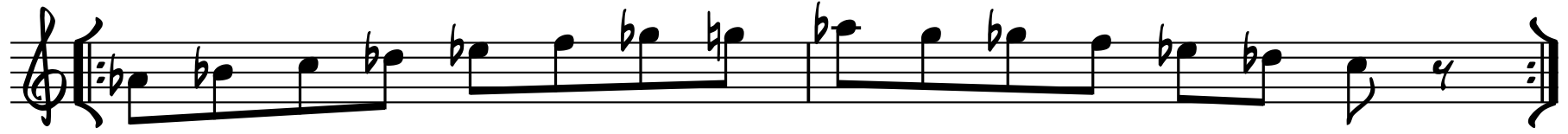




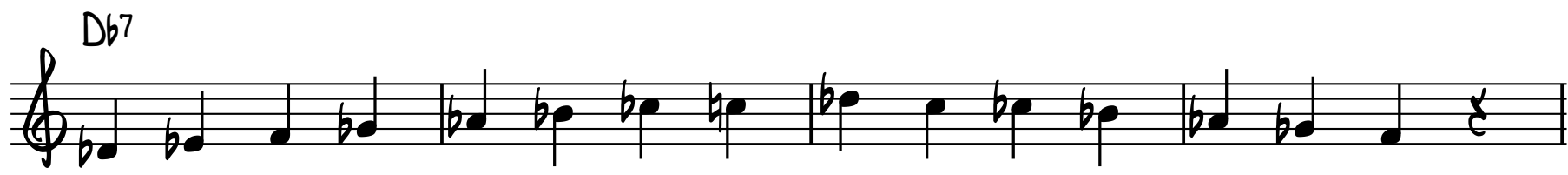

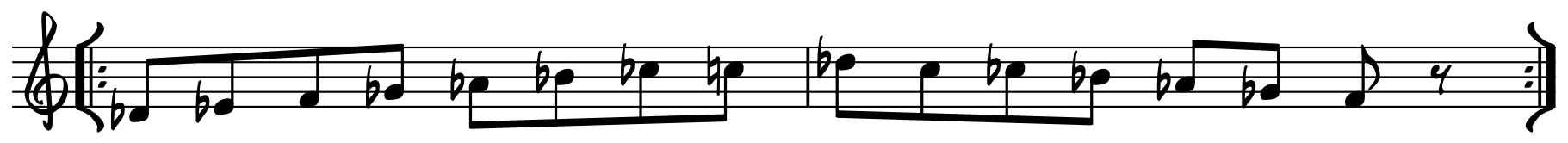

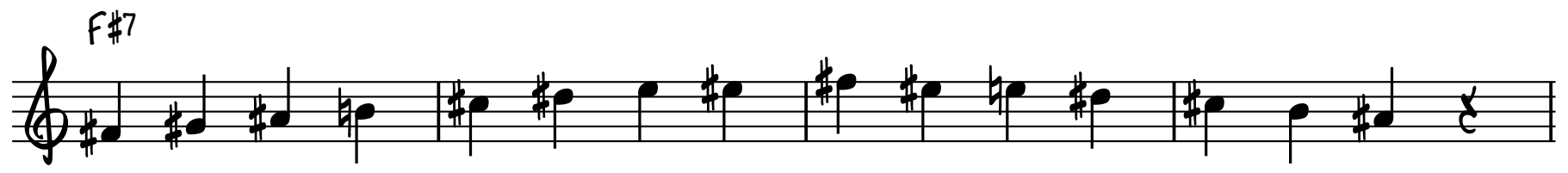

舟( $b^{B 7}$

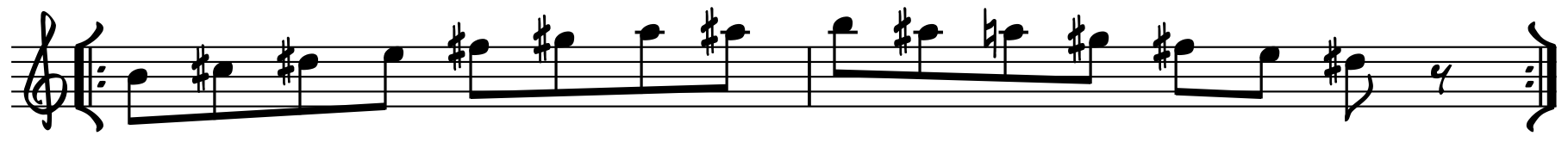

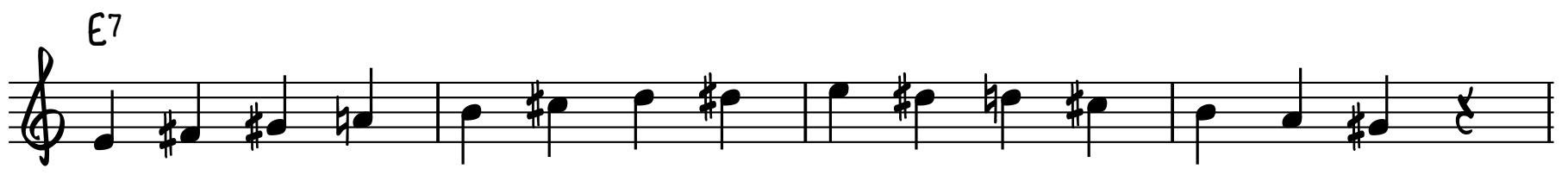

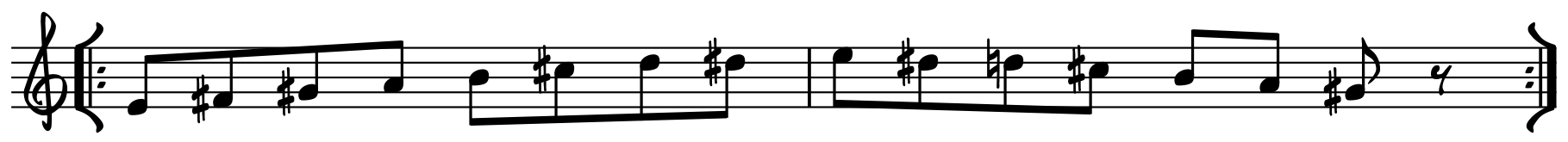
$b^{A 7} \cdot \ldots$

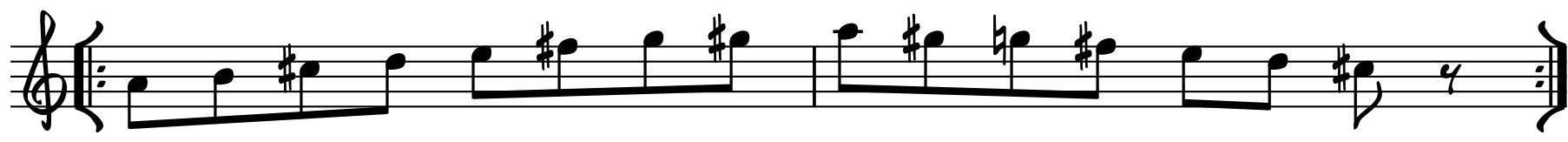



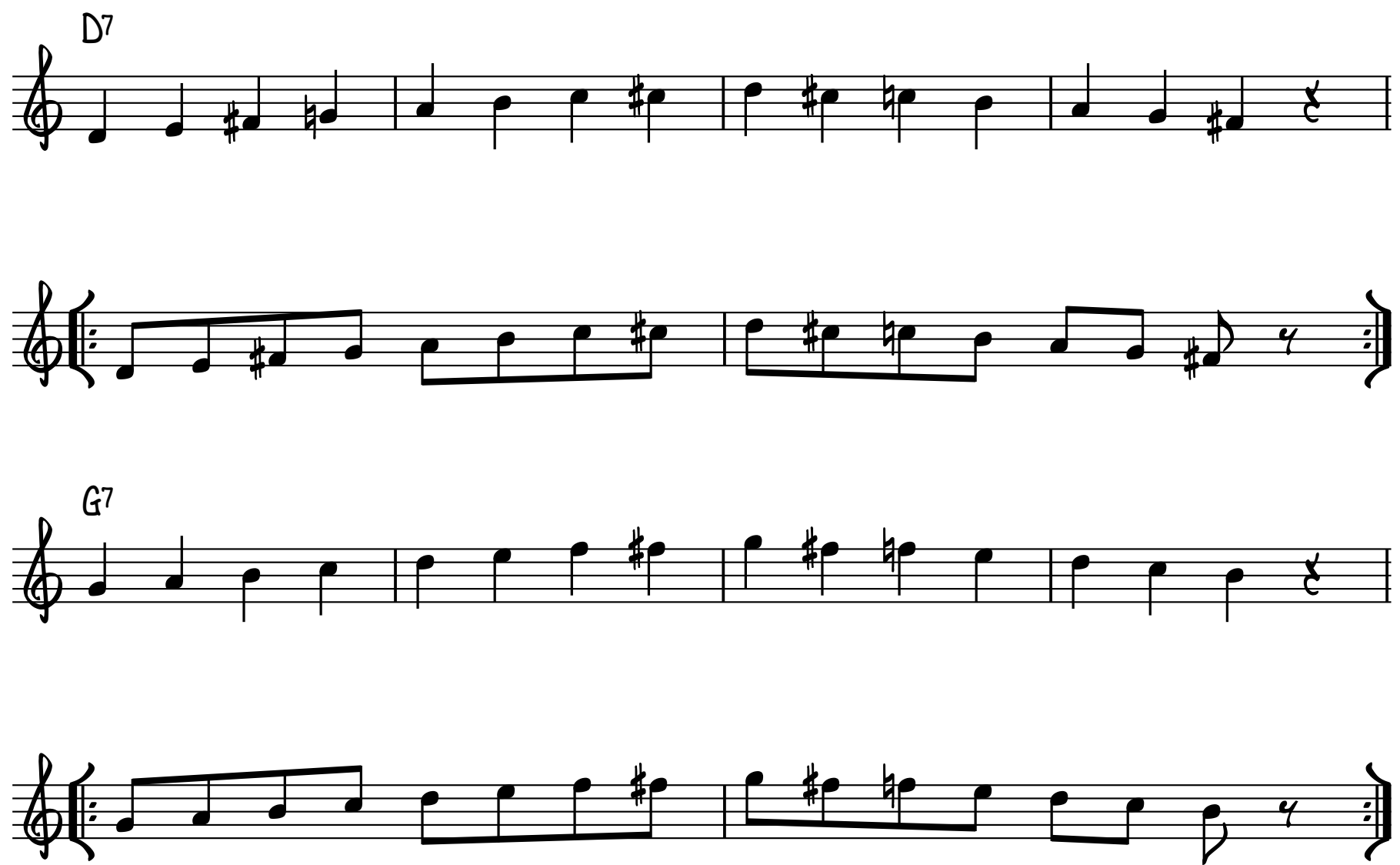

ONCE YOU HAVE MASTERED THIS AT THE FASTEST TEMPO, TRY TO PRACTICE THESE SCALES STARTING ON THE 3RD, 5TH AND FLAT 7TH SCALE DEGREES.

C7

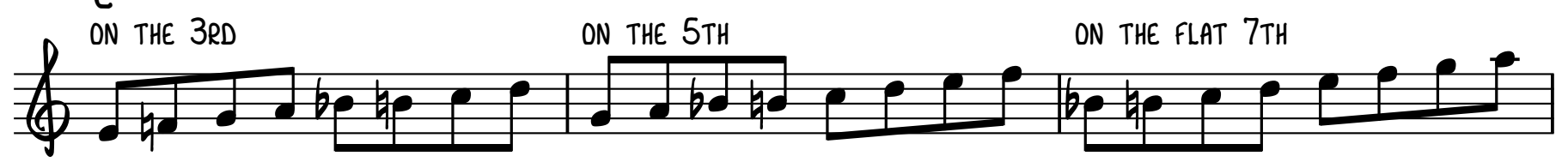




\section{SCALE EXERCISE 3 - MINOR BEBOP SCALES}

TRACK 19 - 112 BPM

TRACK 20 - 132 BPM

TRACK 21 - 152 BPM

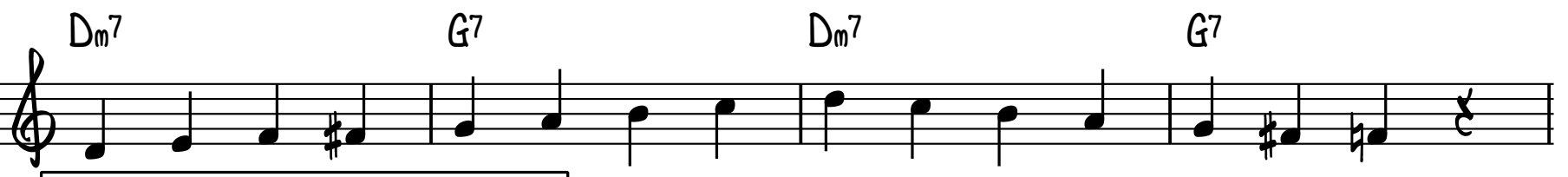

PLAY ALL SUCCESSIVE 8TH NOTES WITH

THIS ARTICULATION (NOTICE THE MAREING "SIMILE")
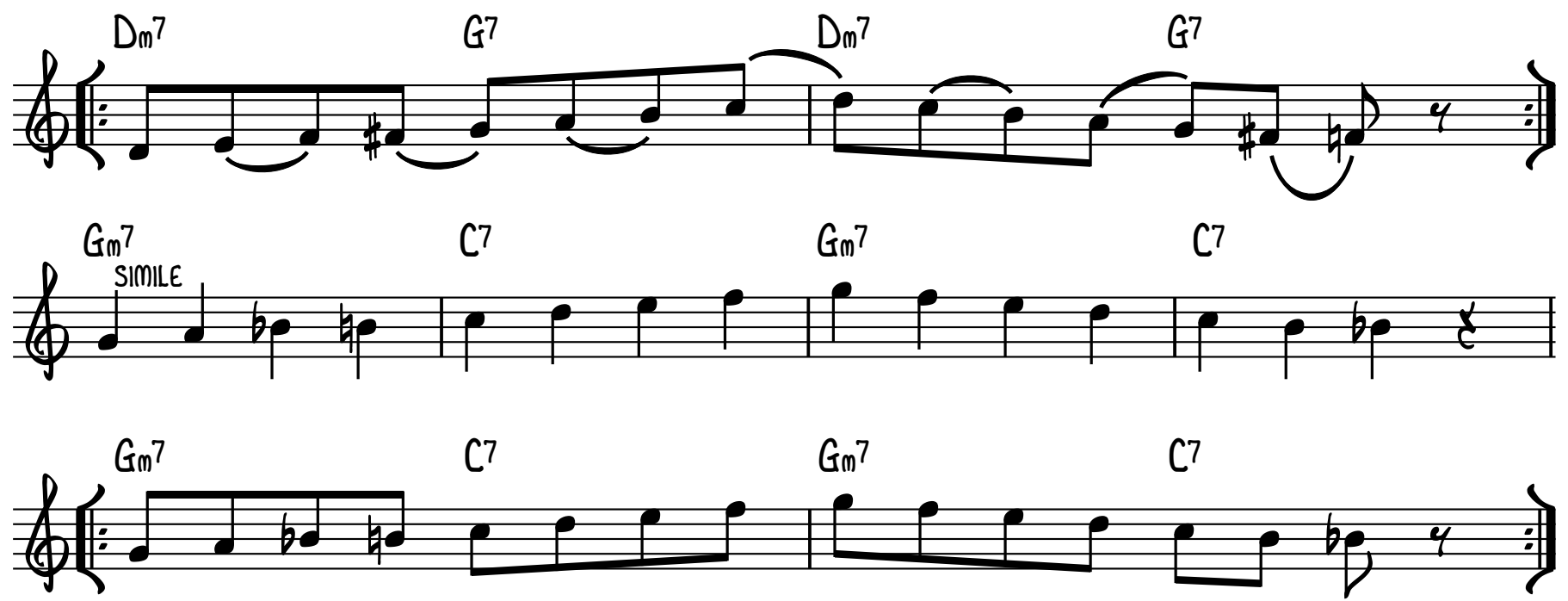

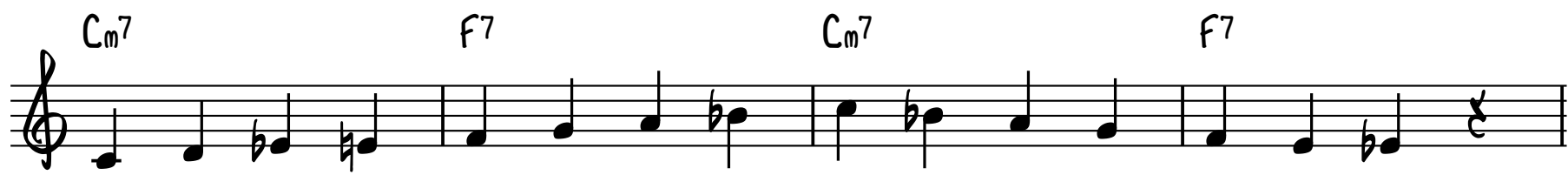

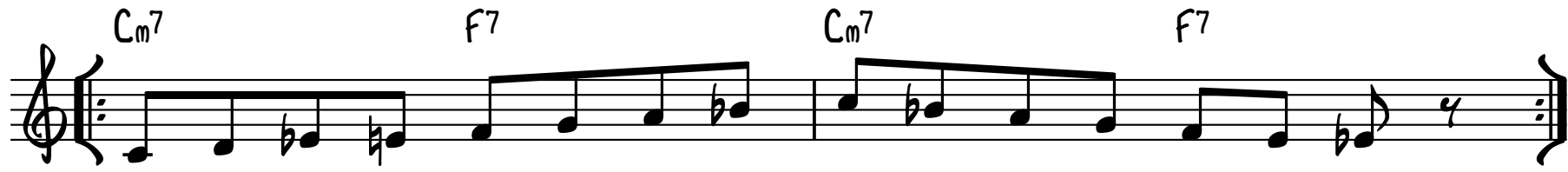

$\left.\right|^{F_{m}{ }^{2}}$

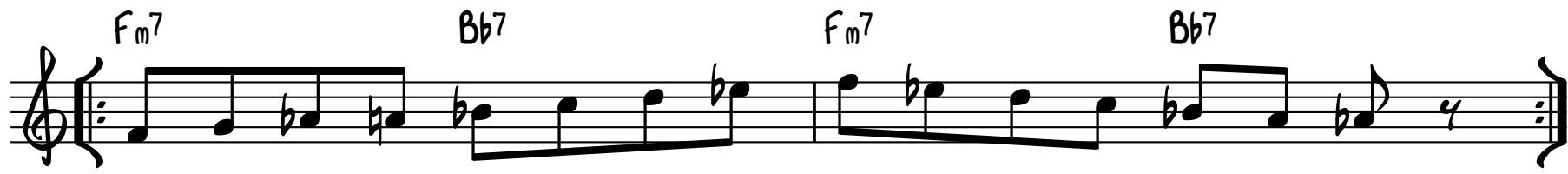

$\left.\right|^{B b b^{7}}$ 


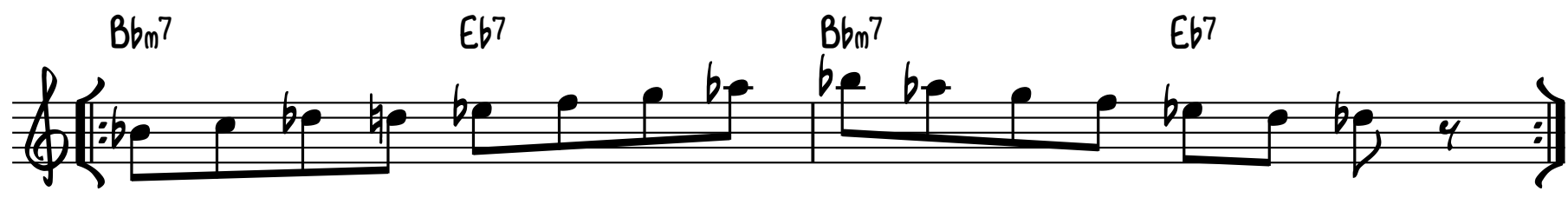

${ }^{E b_{n} 7}$

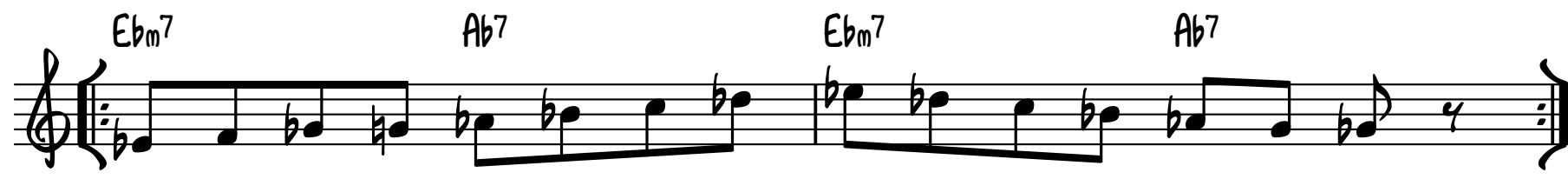

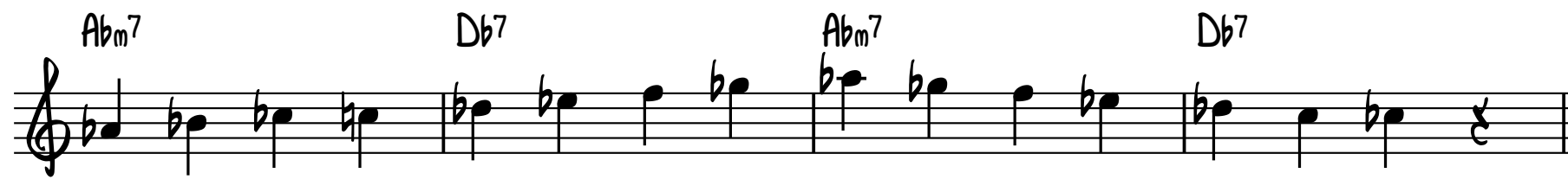

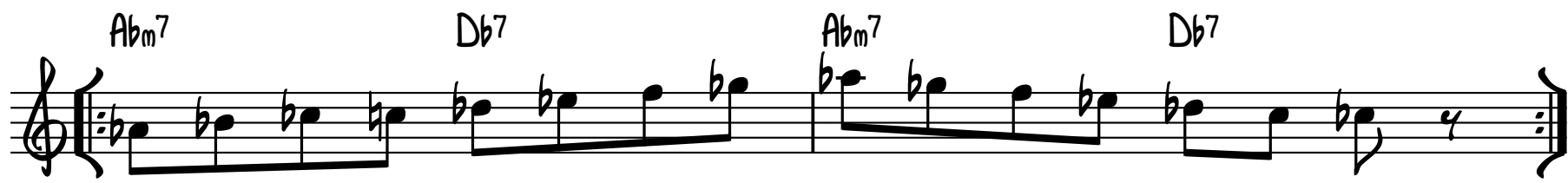

$\begin{array}{llll}D b m^{7} & G b 7 & D b m & G b 7\end{array}$

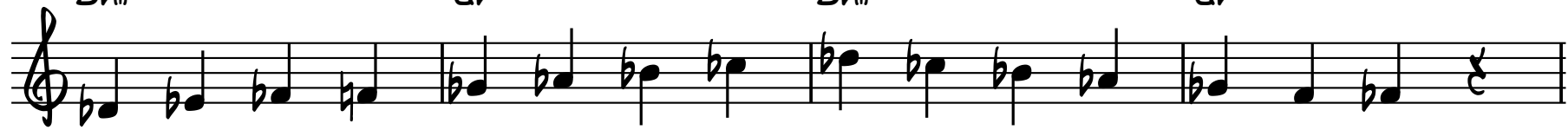
$D \mathrm{~m}^{7}$
$G 67$
$D b_{m} 7$
$G 67$

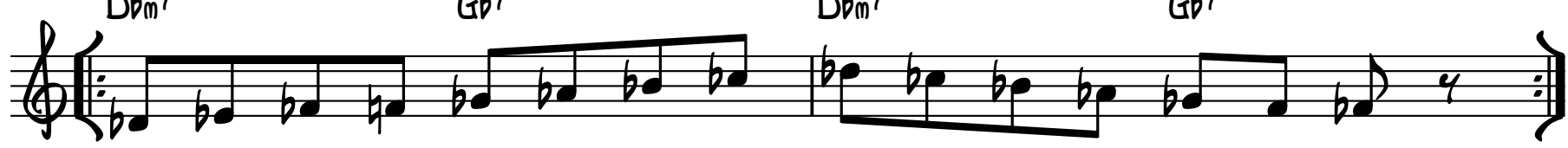

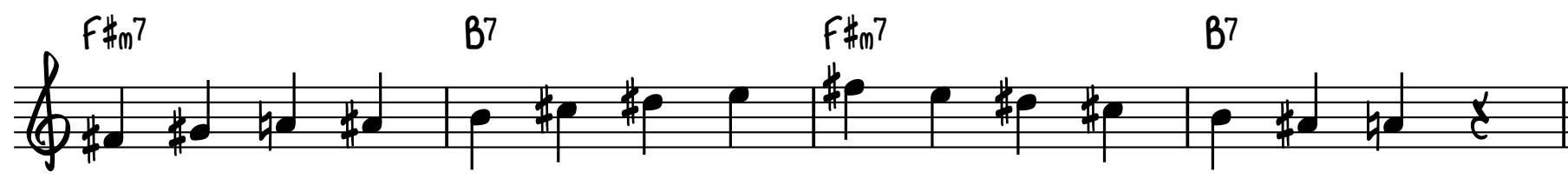

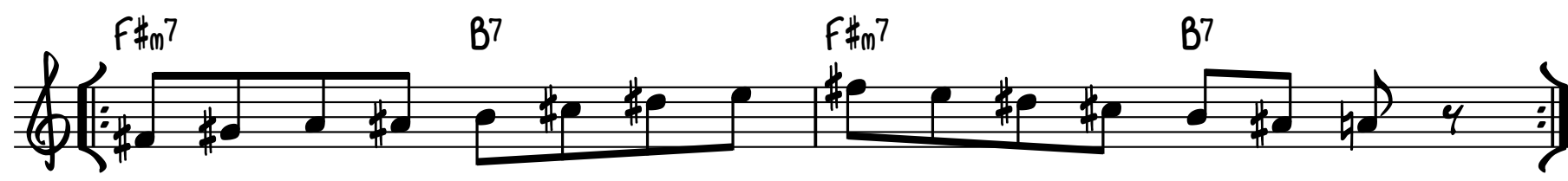

$b^{B_{07}^{7}}$ 

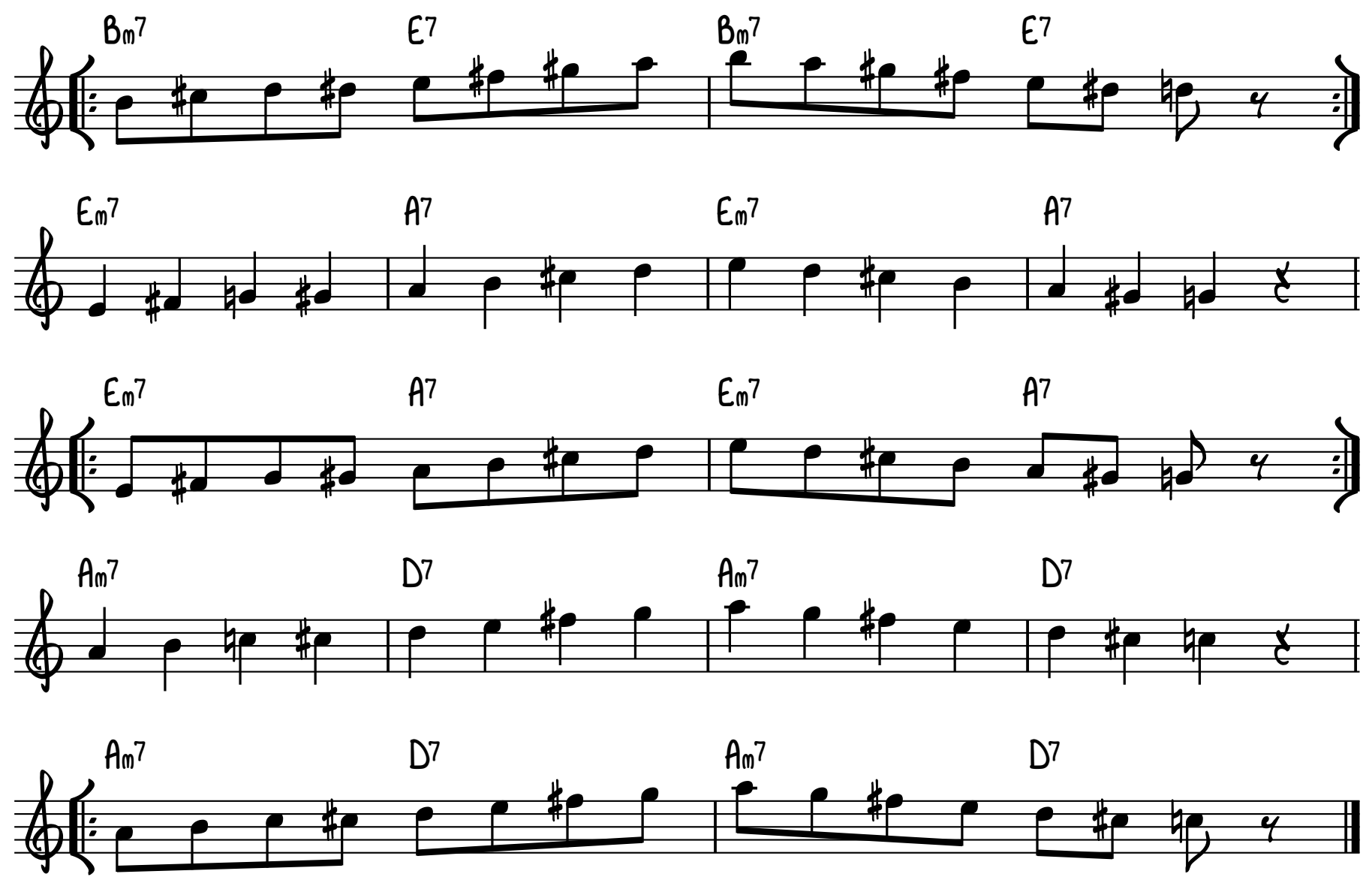

THIS IS THE 5TH MODE OF THE DOMINANT BEBOP SCALE. FOR FURTHER exploration, ReFER to the expansion In SCALE EXERCise 2. 


\section{ONE BAR TWO FIVE}

SWING

TRACK 22 - 100 BPM

TRACK 23 - 120 BPM

Gm7 C7 Fmaj7 $\quad F_{m}^{7} \quad$ Bb7 EbMAj7 TRACK 24-140 BPM

$\# 4$ (4)

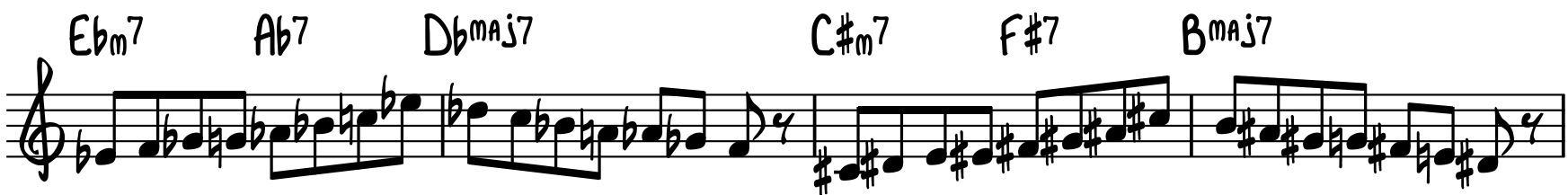

$\mathrm{B}^{\mathrm{Bm}^{7}} \mathrm{E}^{\mathrm{Amaj7}} \mathrm{Am}^{\mathrm{m}} \mathrm{D}^{7}$

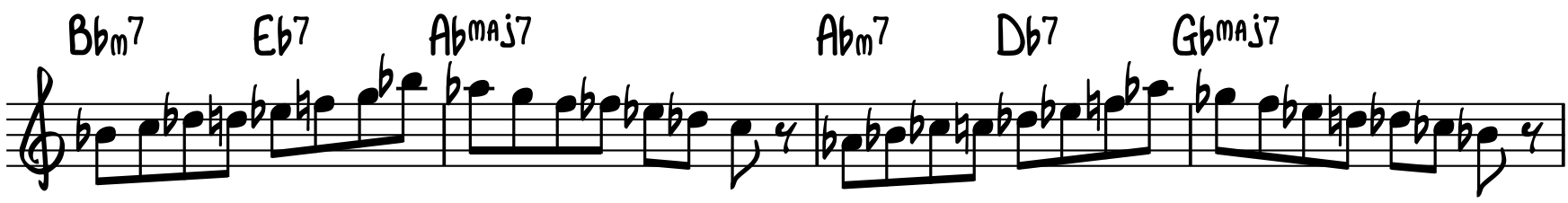

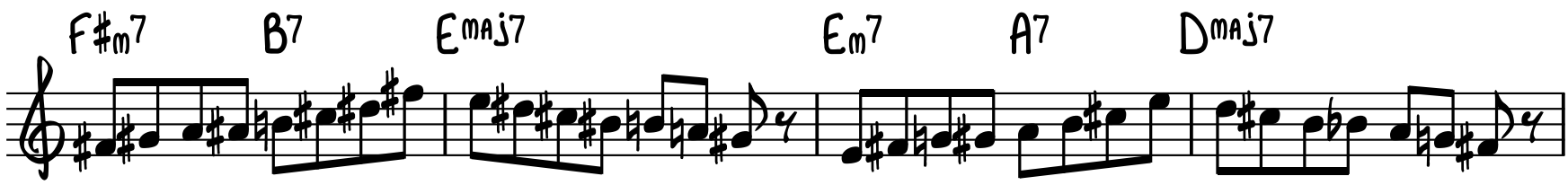

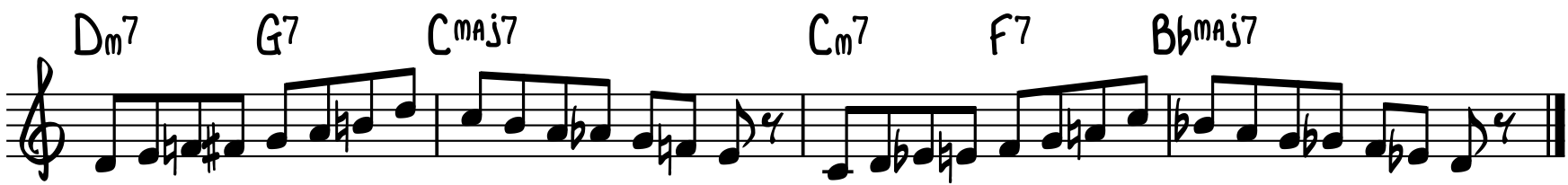




\section{TWO BAR II-V}

$\overbrace{}^{\mathrm{Am}^{7}} \mathrm{D}^{7}$

$f^{\mathrm{Gm} 7}{ }^{C 7}$

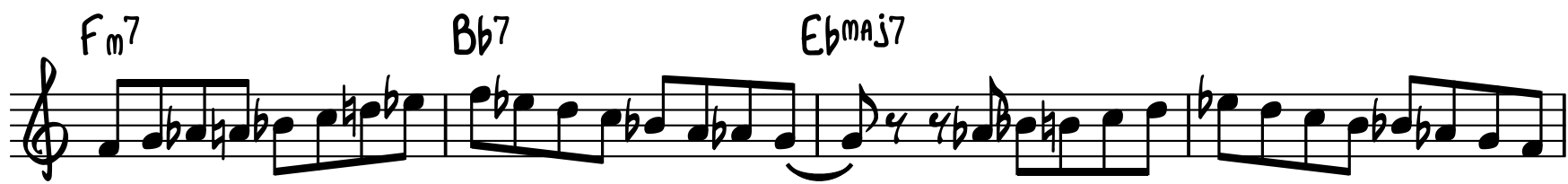

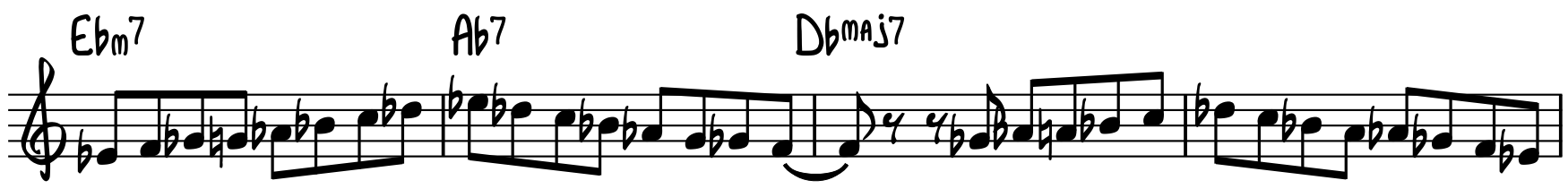

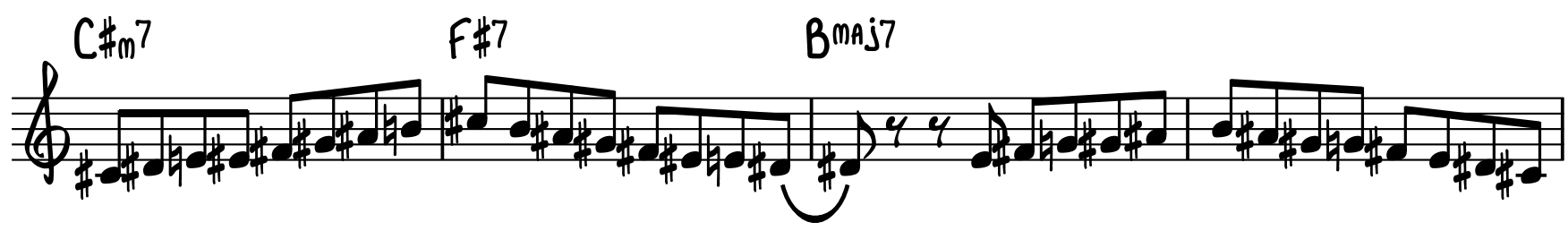

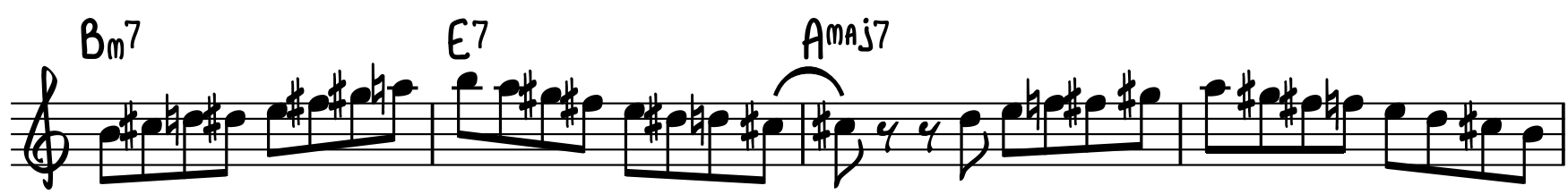

$\mathrm{Cm}_{m} \quad \mathrm{F7} \quad$ Bbmaj7

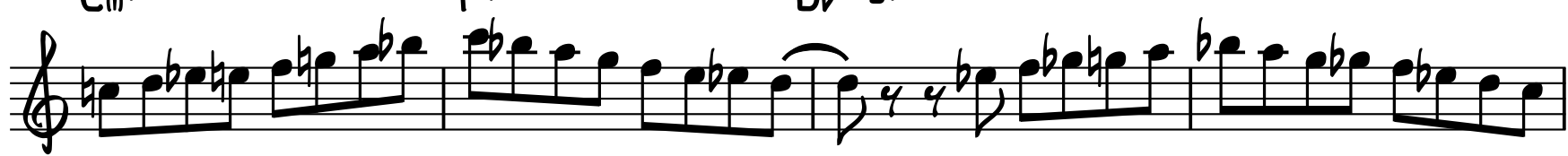




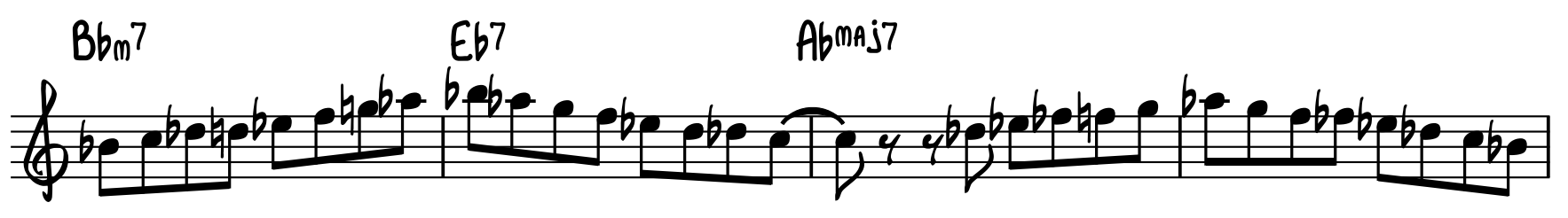

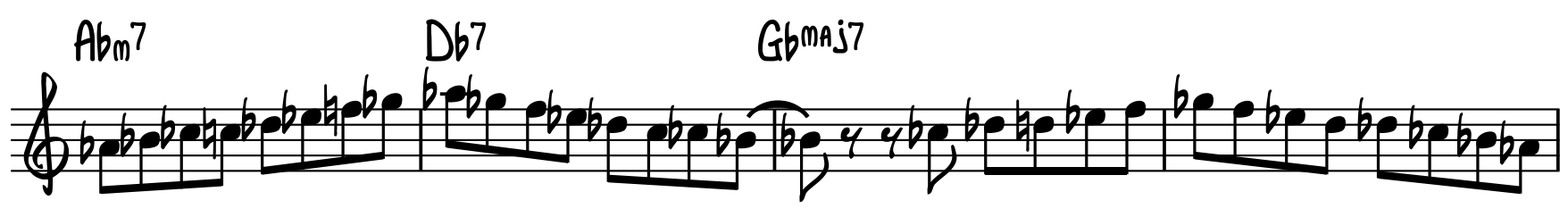

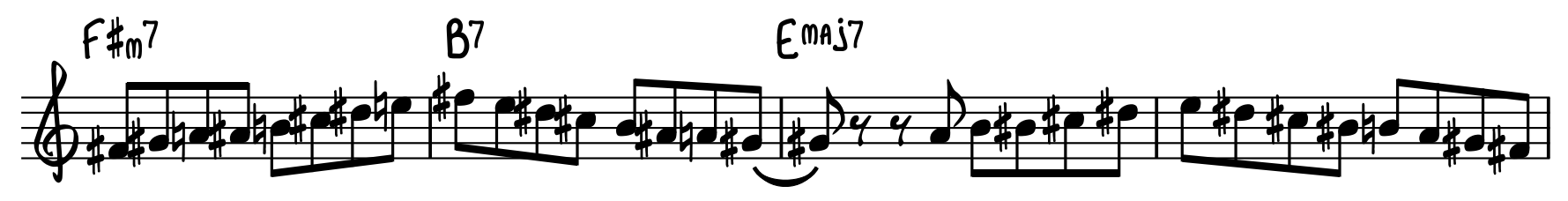

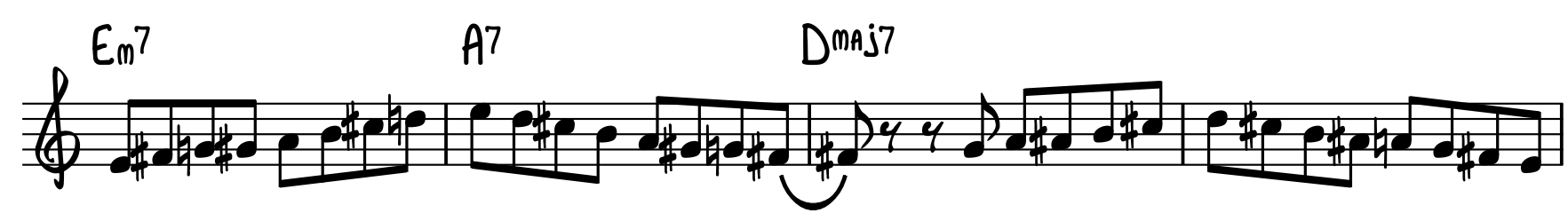

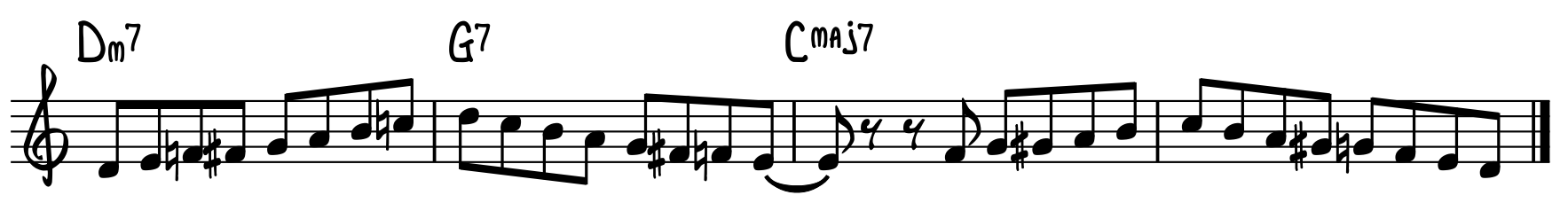


I TO IV MODULATION THROUGH III

TRACK $28-92 \mathrm{BPM}$
TRACK $29-112 \mathrm{BPM}$

TRACK 30 - 132 BPM

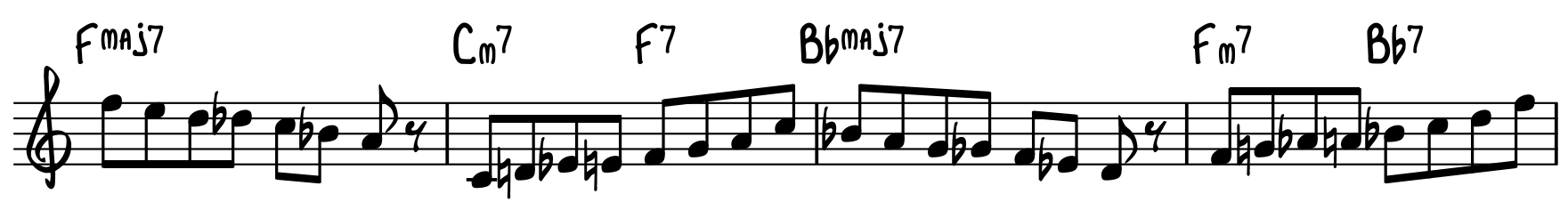

Ebmaj7 Abm $E b 7 \quad A b$ maj $\quad b^{2} \quad A b 7$

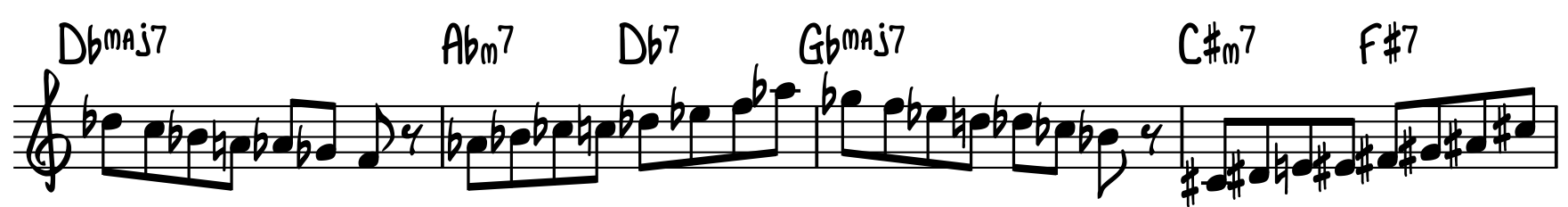

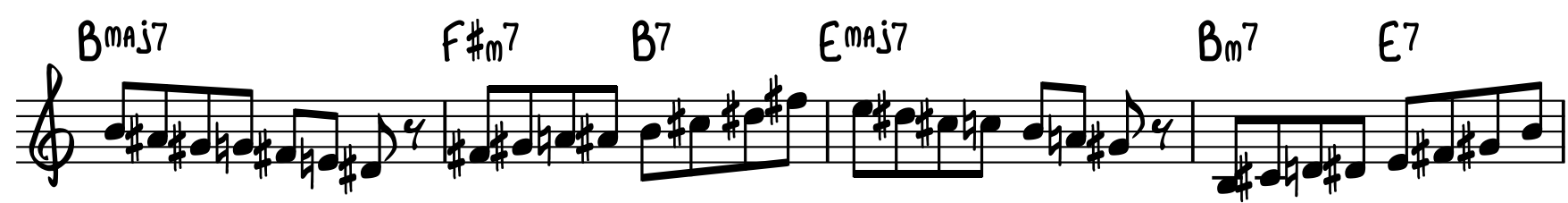

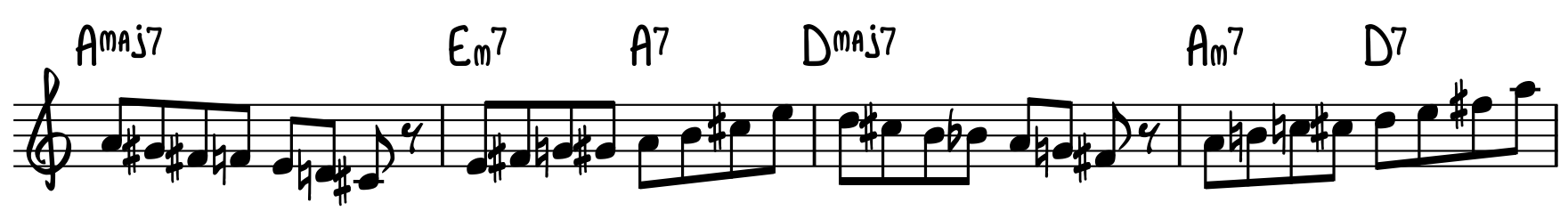

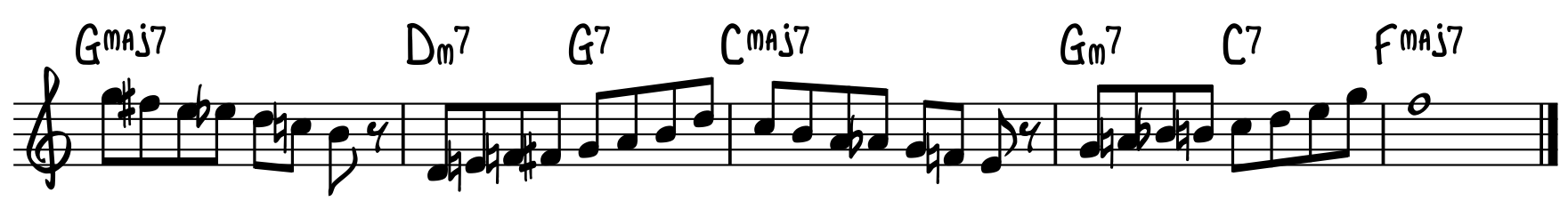


C INSTRUMENTS

\section{ETUDE 1 - MAJOR BEBOPOP}

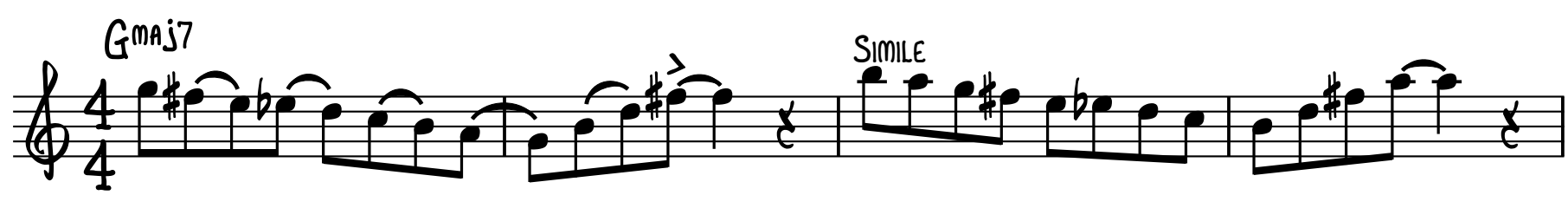

5 Fmaj7

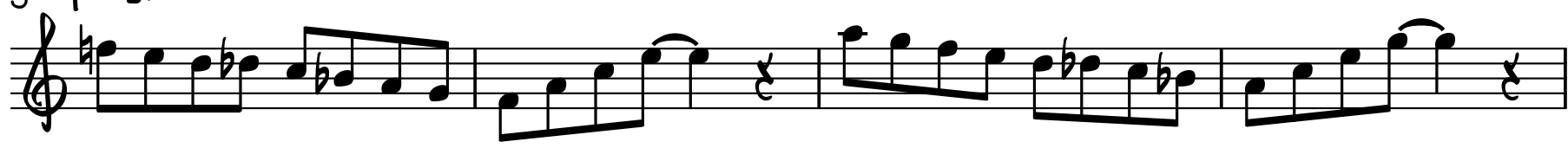

Ebmaj7

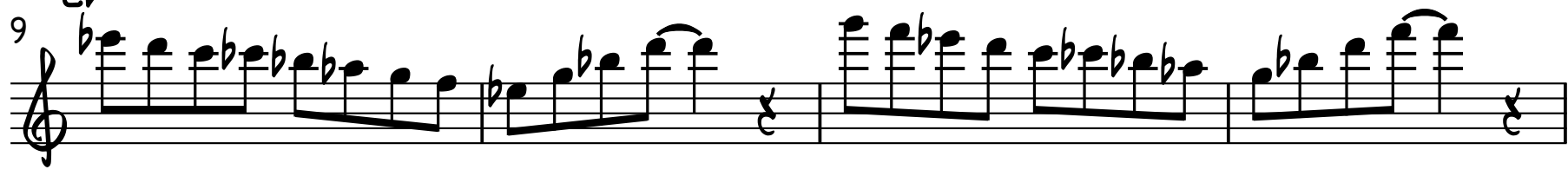

${ }_{13}^{\text {Fmaj7 }}$

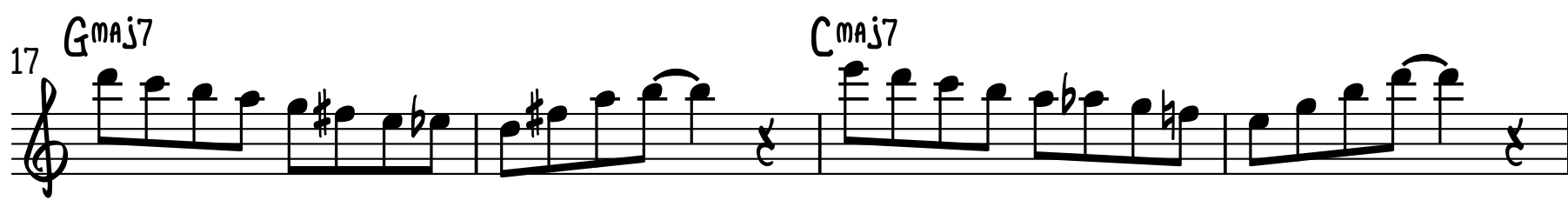

${ }_{21}$ Fmaj7 Bbmaj7

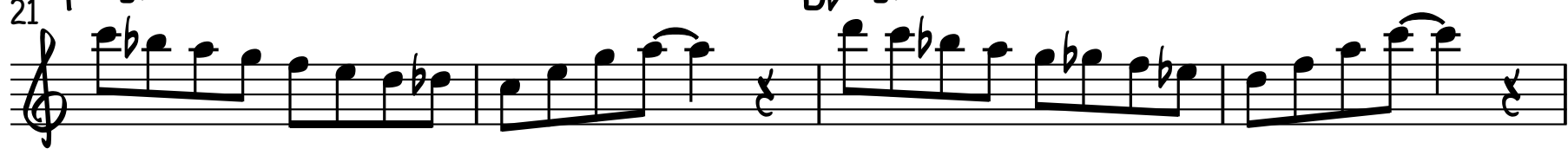

25 Ebmaj7

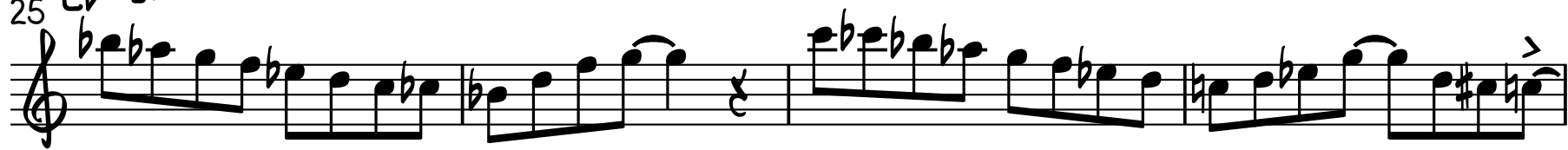

29 Fmaj7

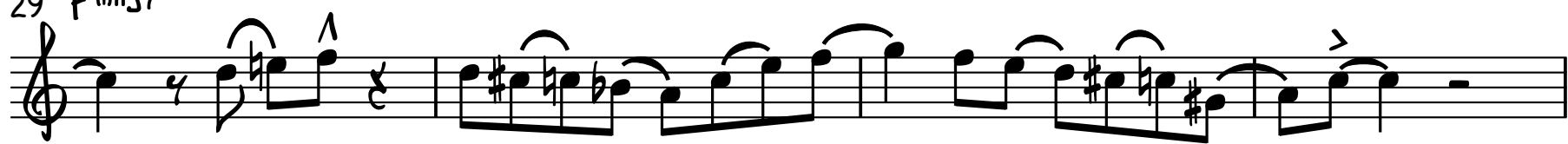


2

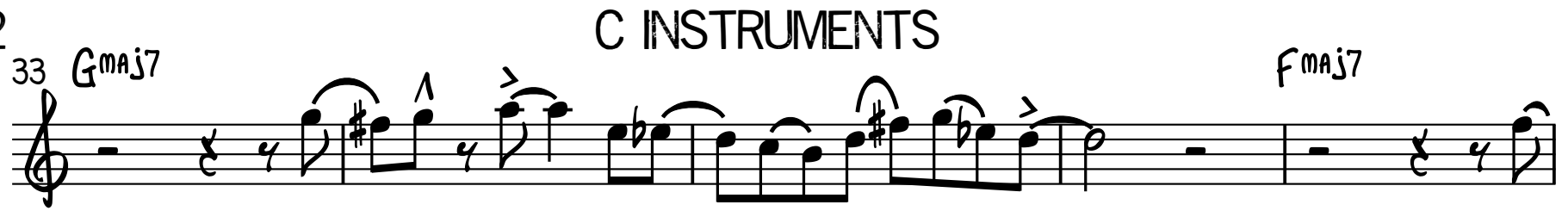

(4)

(4)

(46)

C1: Caj7

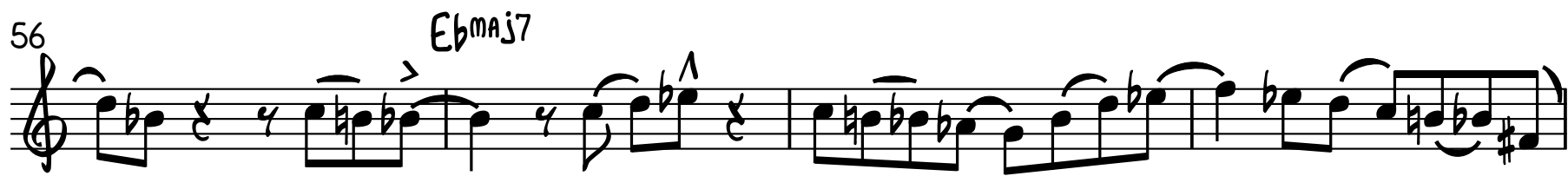

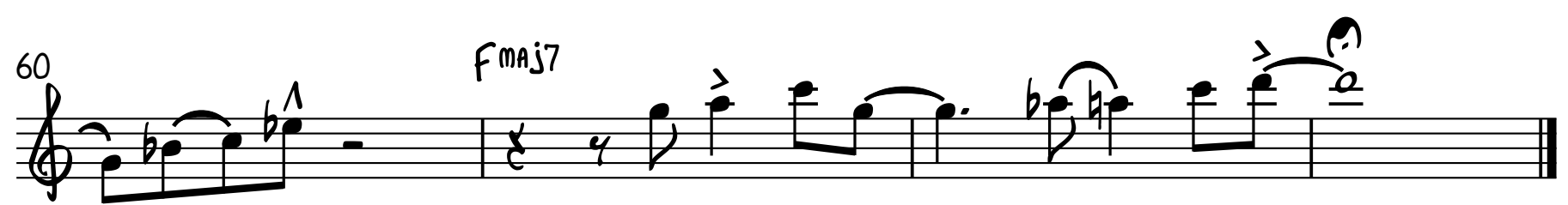


ETUDE 2 - MAJOR BEBOP
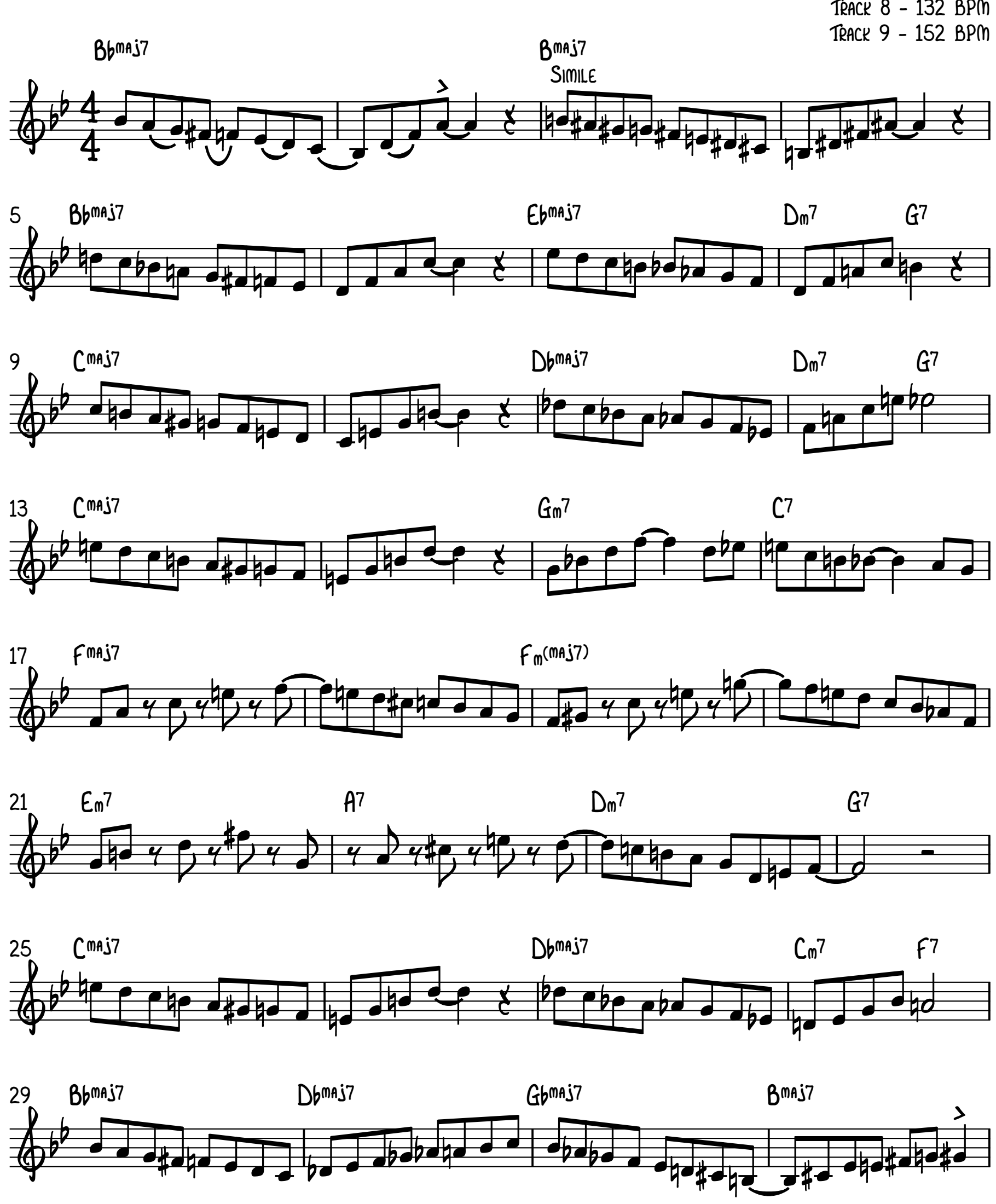
2

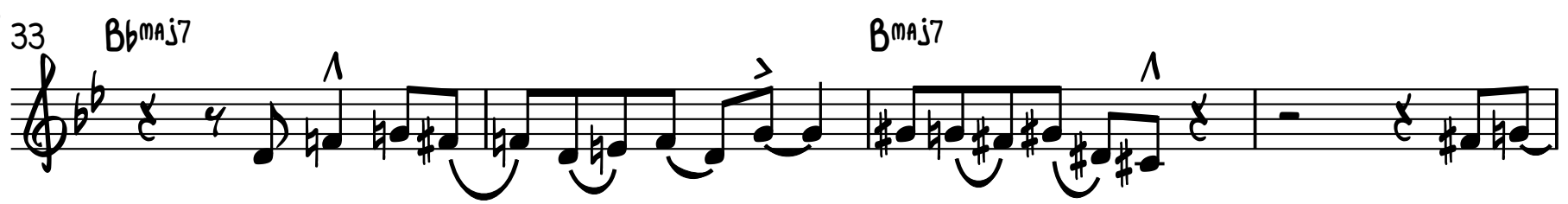

${ }^{37}{ }^{\text {Bmmi7 }}$

$42 \rightarrow D_{6 m a j 7} \quad D_{m} \quad \underset{\Lambda}{G} \quad$ cmaj7

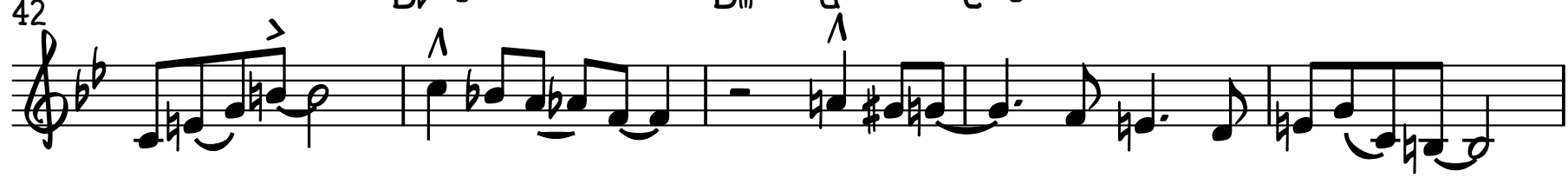

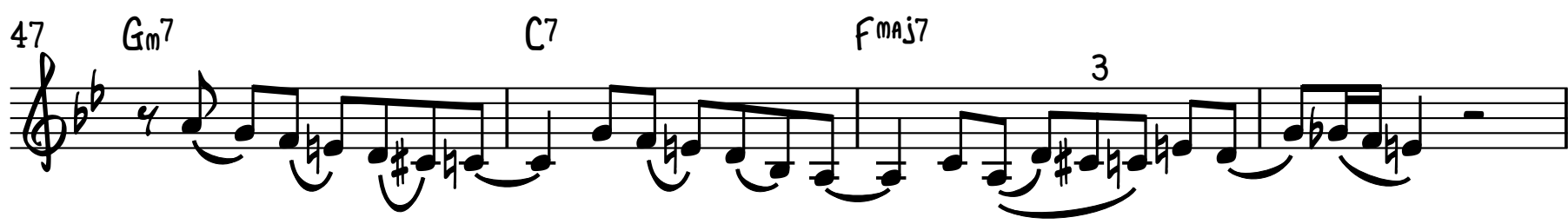

${ }^{51} \mathrm{Fm}^{\text {(mij) }}$

${ }^{55} \mathrm{Dm}^{7}$

${ }^{59}{ }^{\mathrm{Dm} \text { mij7 }}$

62 Dbmaj7 G G maj7 $\quad$ Bmaj7 $\quad B b^{\text {maj7 }}$

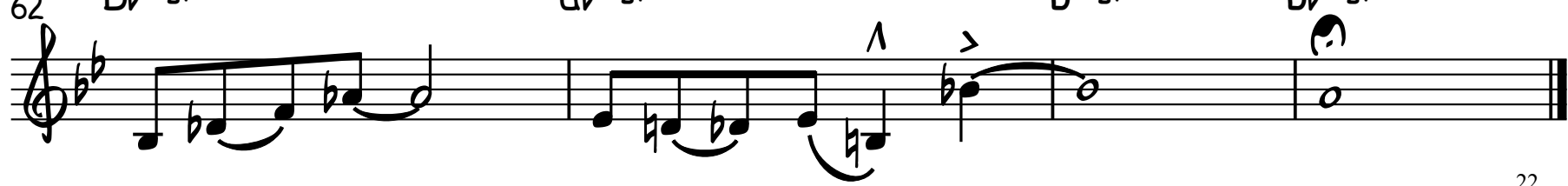




\section{ETUDE 3 - DOMINANT BEBOP}

BOOGALOO:

TRACK $13-132$ BPM TRACK $14-152$ BPM TRACK 15 - 172 BPM
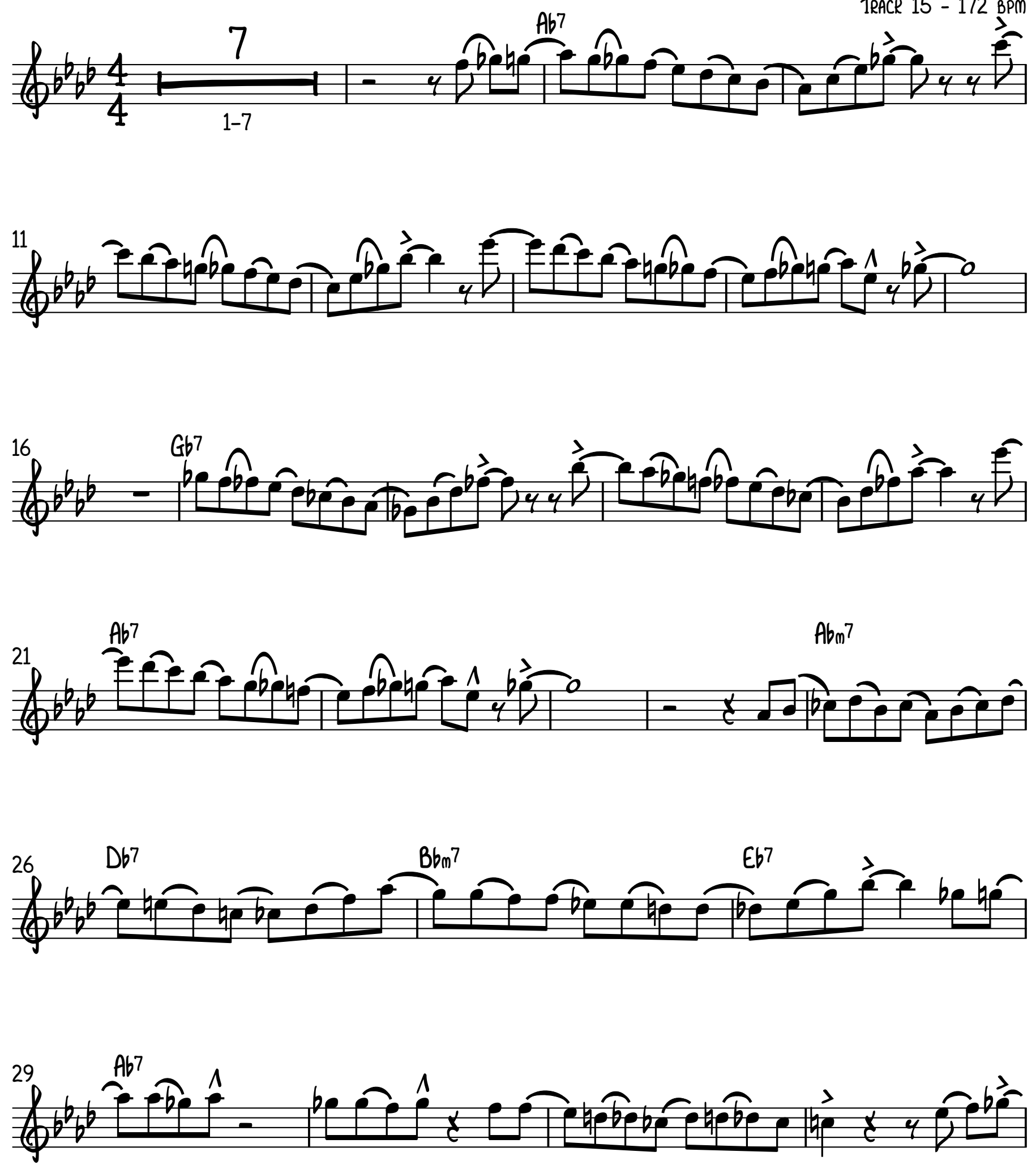
2

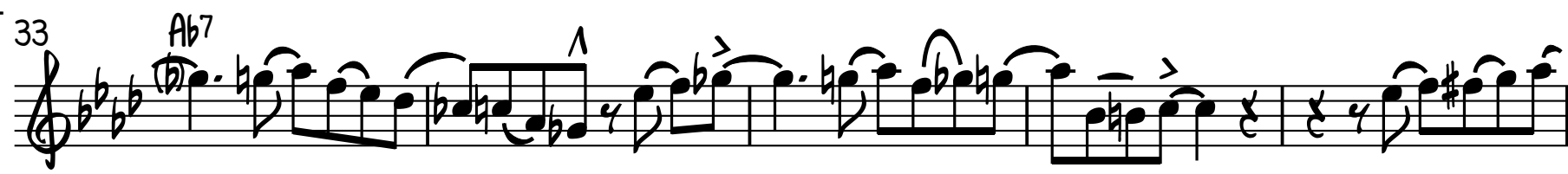

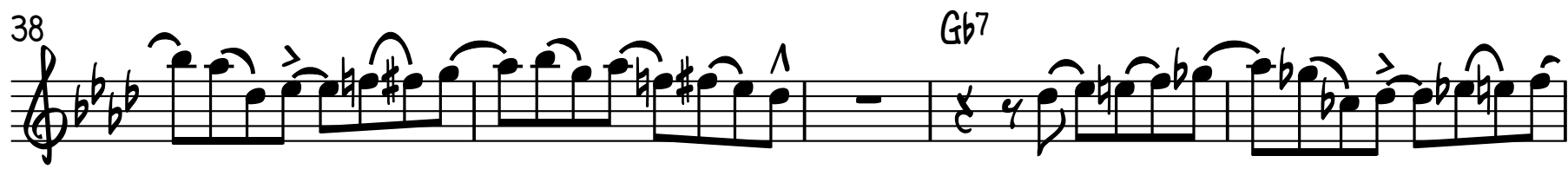

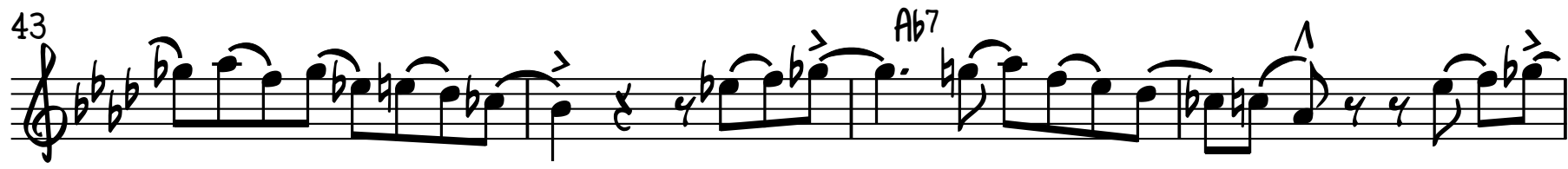

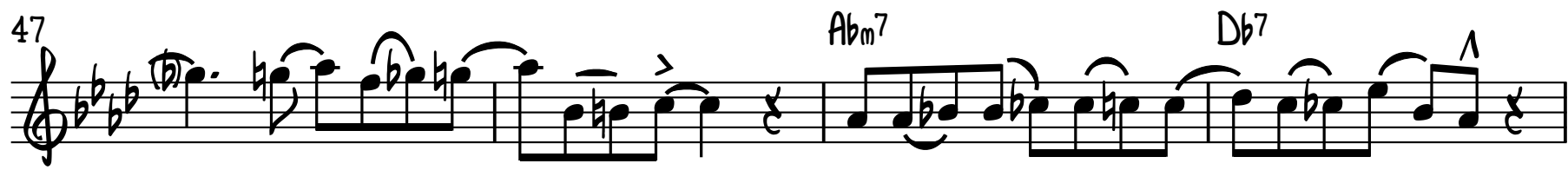

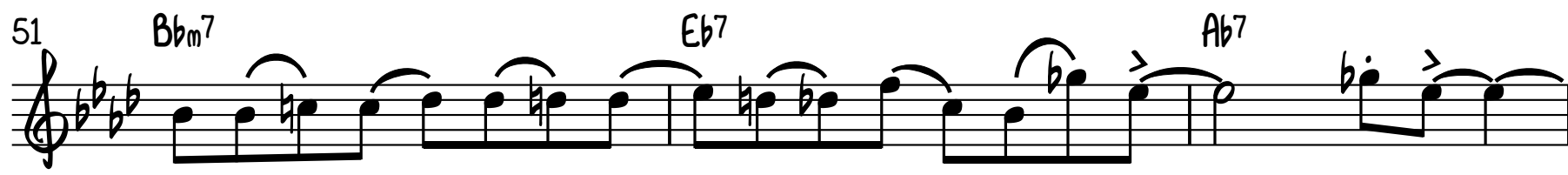

$\left.\right|_{\frac{b}{b}} ^{54}$ 


\section{ETUDE 4 - DOMINANT BEBOP}

SWING

TRACK 16 - 112 BPM

$4_{4}^{4}$

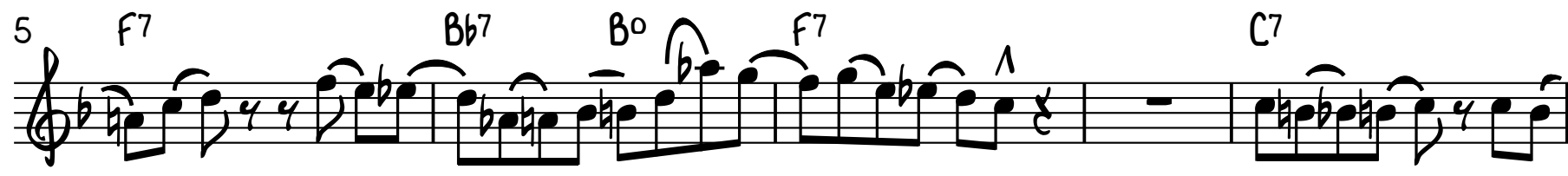

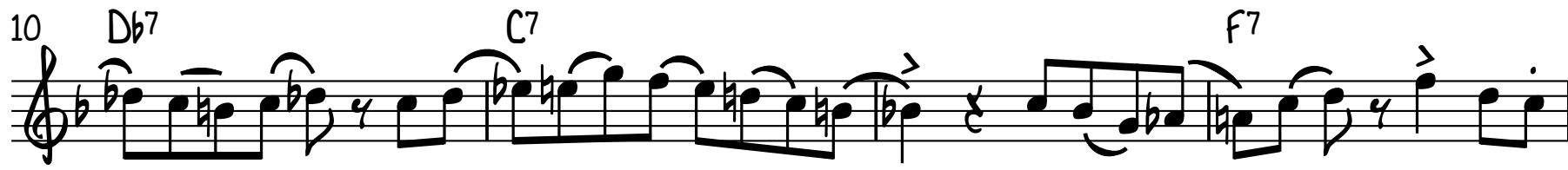

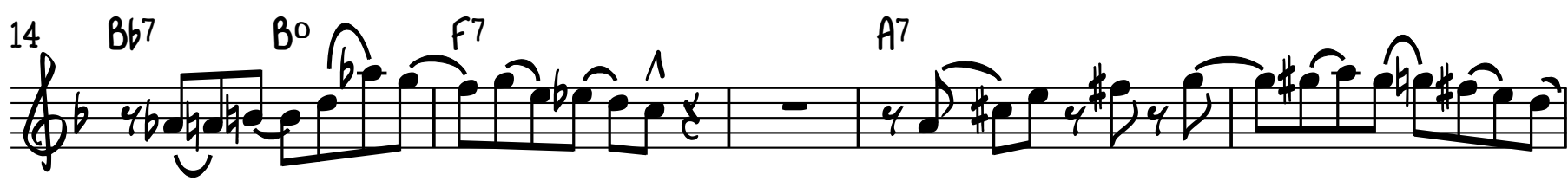

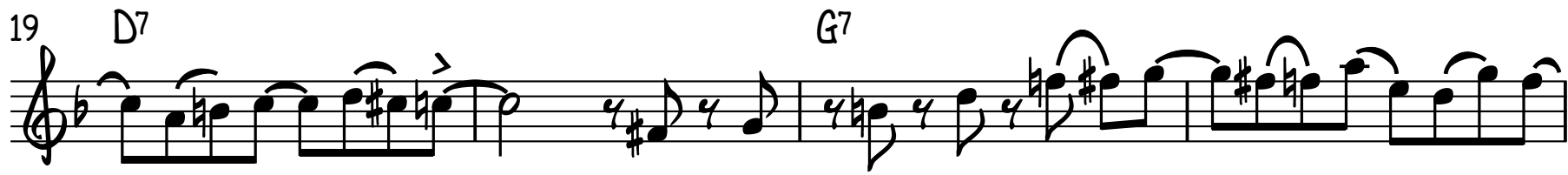

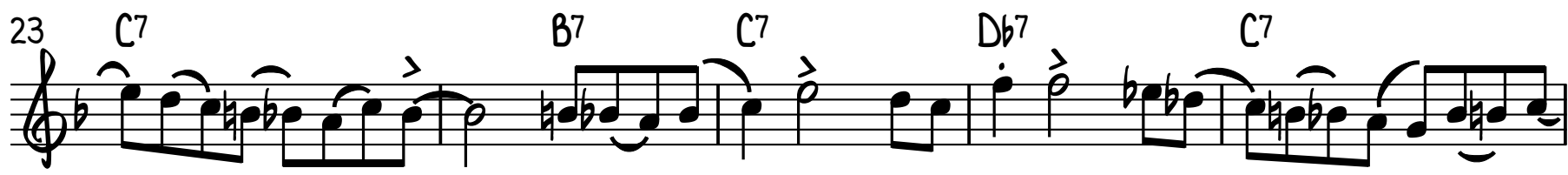

(63\% 
2

23

(7)

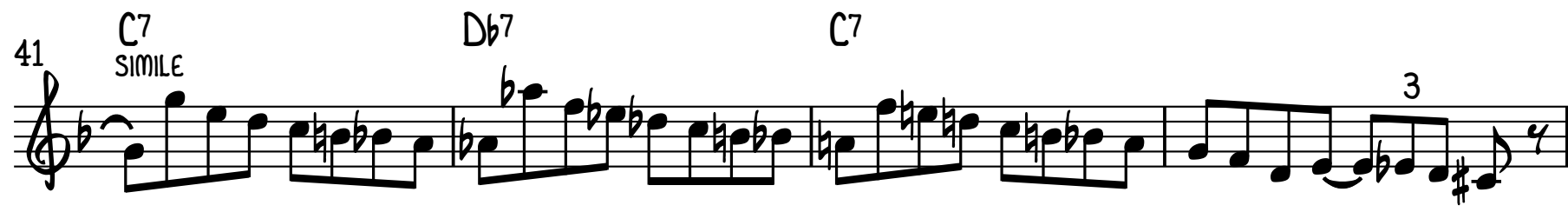

(4)

19.

(5)

(5)

(0) 
C INSTRUMENTS

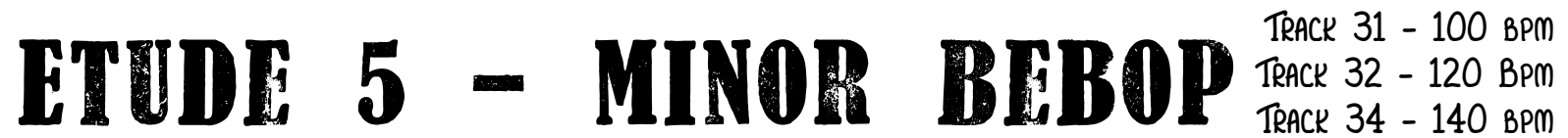

$4_{4}^{4}$

(Am

$D_{m}{ }^{G 7} D_{m}{ }^{7}$

$13{ }_{1}^{A m 7}$

(17)

(4)

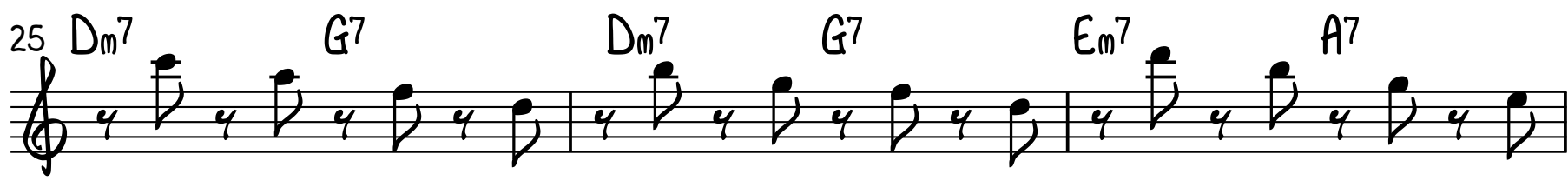

$\begin{array}{lllllll}E_{m} 7 & A 7 & A_{m} 7 & D^{7} & A^{7} b_{m} & D b 7 & \text { Cmaj7 }\end{array}$

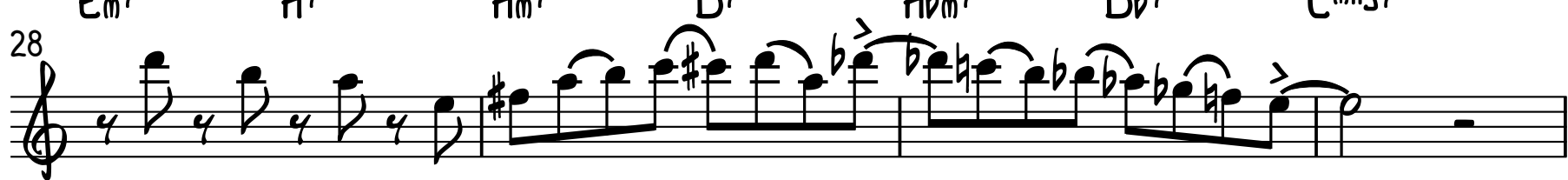


C INSTRUMENTS

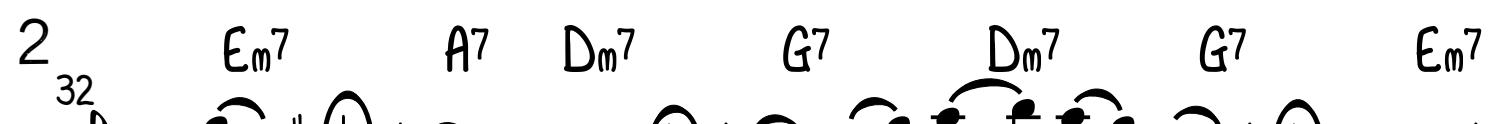

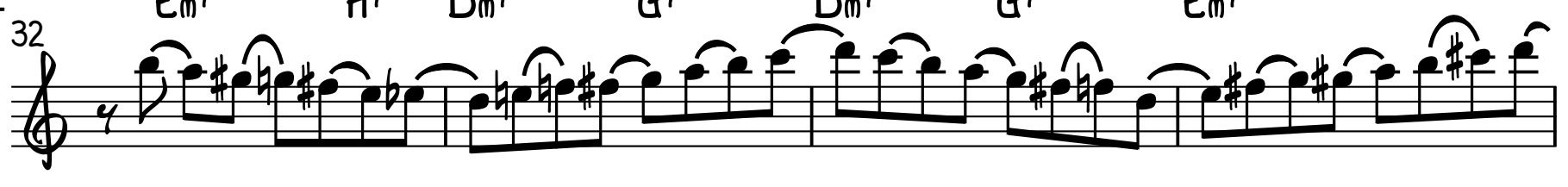

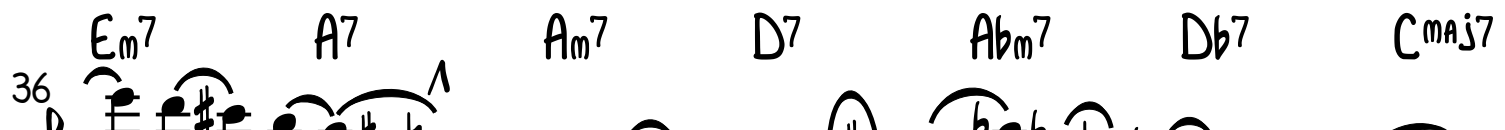
夷 $\begin{array}{llllllll}E_{m}{ }^{7} & A^{7} & D_{m}{ }^{7} & G^{7} & D_{m}{ }^{7} & G^{7} & E_{m}{ }^{7} & A^{7}\end{array}$ 每 $\begin{array}{lllllll}E m^{7} & A^{7} & A_{m}{ }^{7} & D^{7} & A b m^{7} & D_{67} & C^{\text {maj7 }}\end{array}$

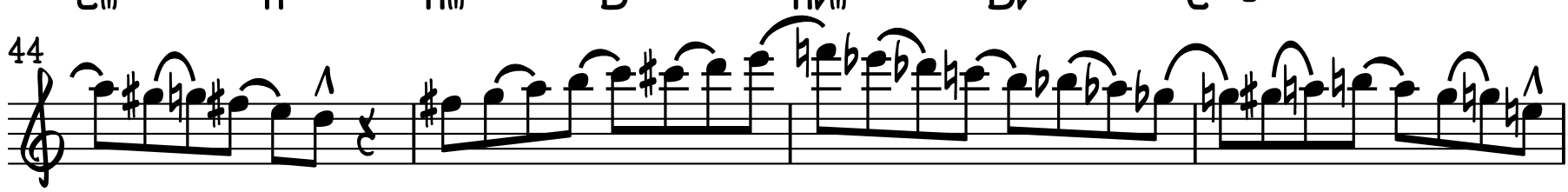
${ }^{48}$ 52. $\quad A_{0}=A^{7} \quad D^{7} \quad A m^{7} \quad D^{7} \quad G^{7}$

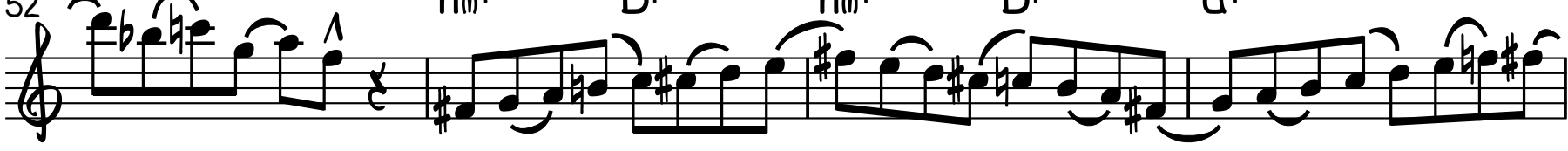
${ }^{56} \mathrm{Dm}^{7} \mathrm{G}^{7} \mathrm{Dm}^{7} \mathrm{G}^{7} \mathrm{Em}^{7} \mathrm{~A}^{7}$ $60 \stackrel{E_{m}{ }^{7}}{A 7} \quad A^{7} \quad D^{7}$

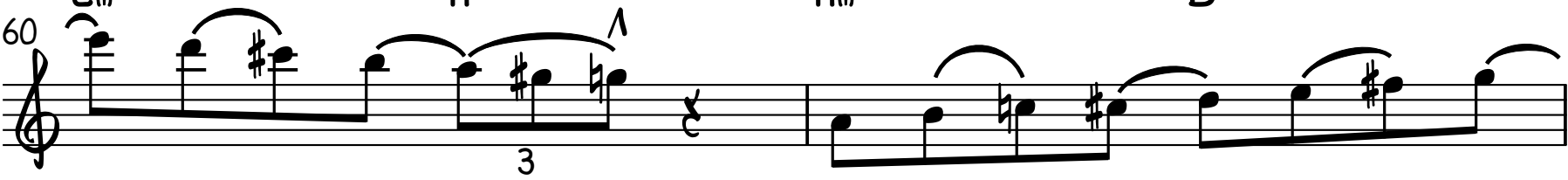
${ }_{4}^{62} \mathrm{Abm}^{7}$ 
Exercises in Developing Bebop Scale Language

for Collegiate Jazz Students

\author{
Lars Swanson \\ West Virginia University
}

\author{
Alto Sax Edition
}




\section{SCALE EXERCISE 1 - MAJOR BEBOP SCALES}

TRACK 1 - 100 BPM

TRACK 2 - $120 \mathrm{BPM}$

Amaj7

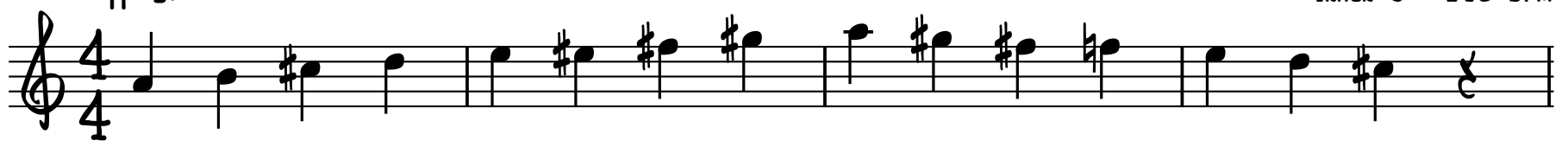

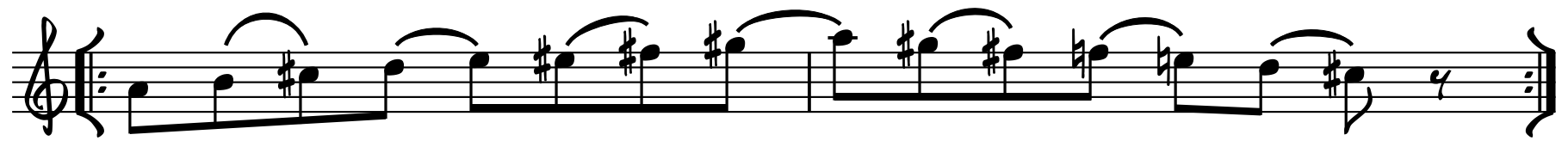

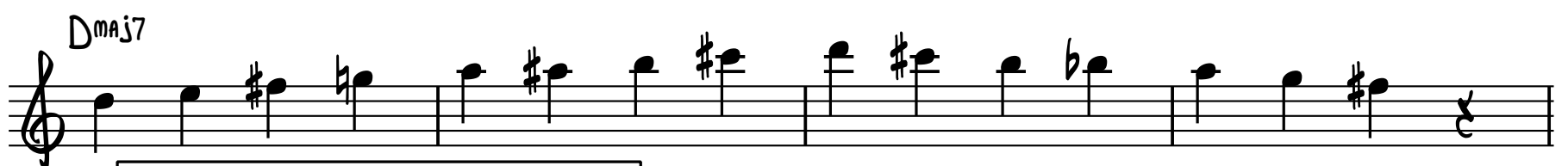
PLAY ALL SUCCESSIVE 8TH NOTES WITH
THIS ARTICULATION (NOTICE THE MARRING "SIMILE")

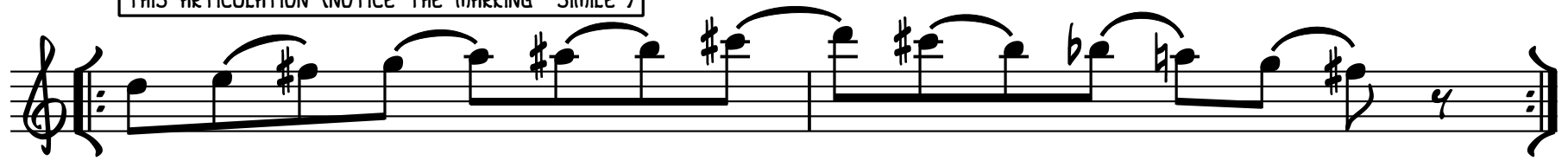

Gmaj7

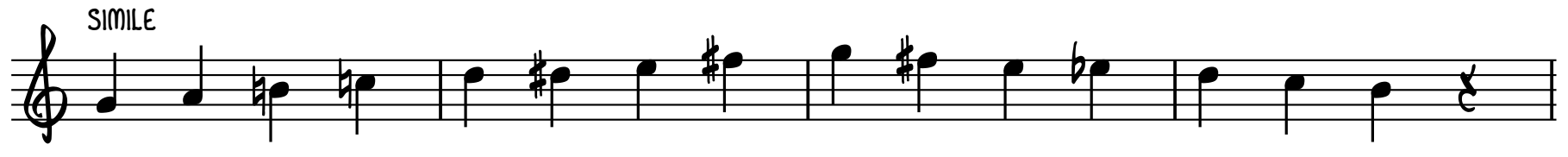

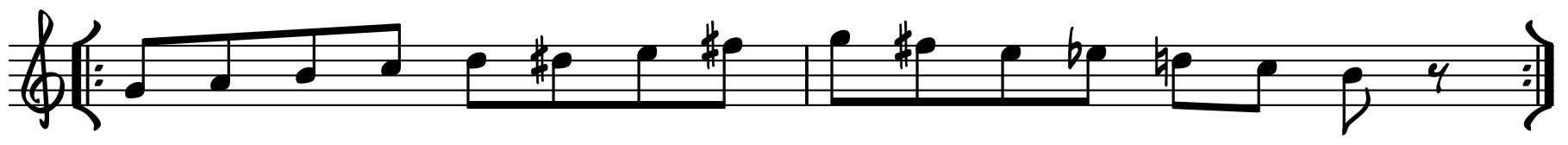

$\oiint^{\text {CMAj7 }}$

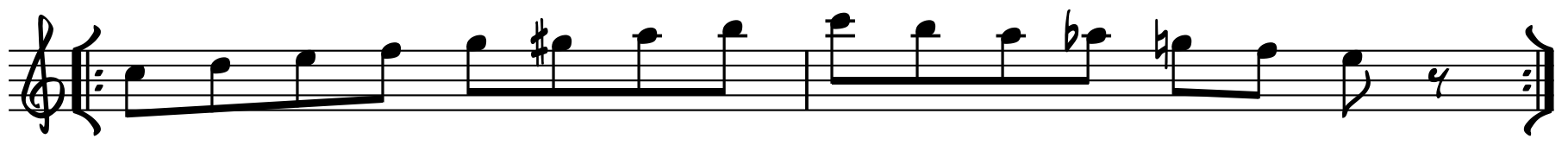

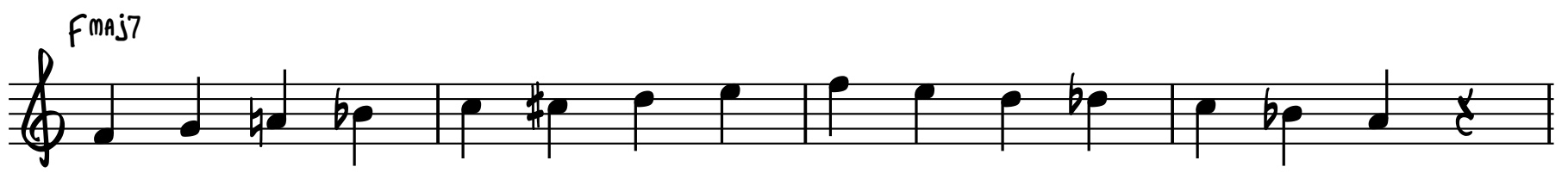




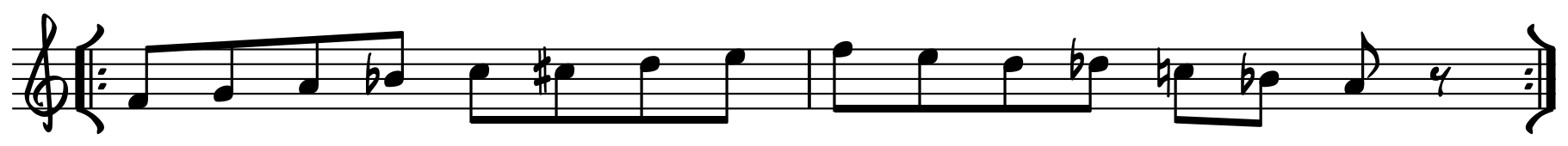

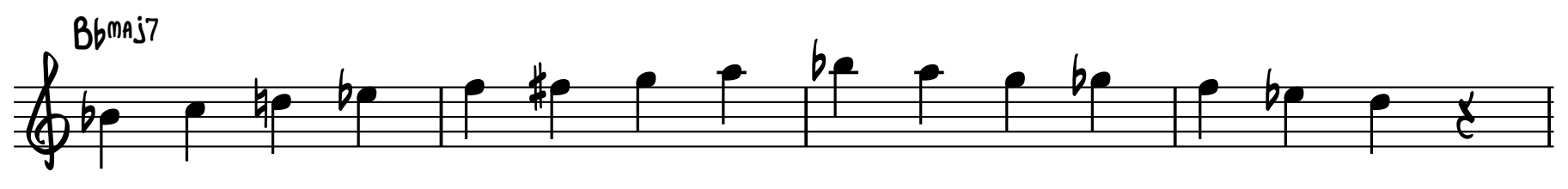

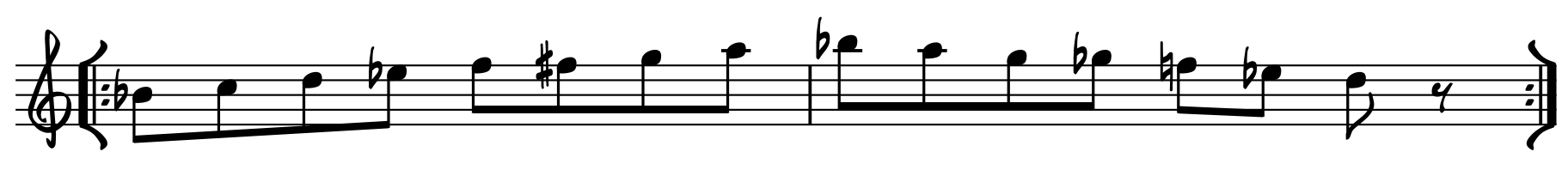
Emonis

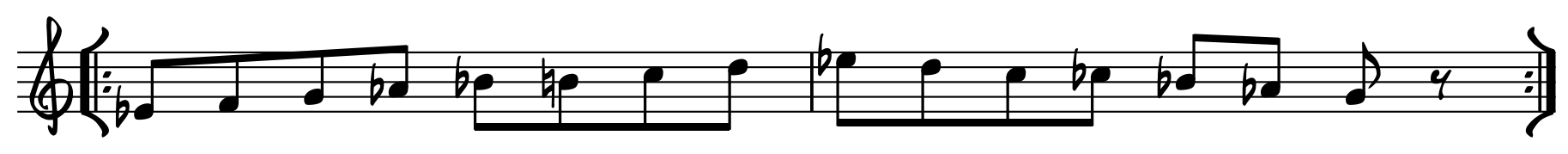

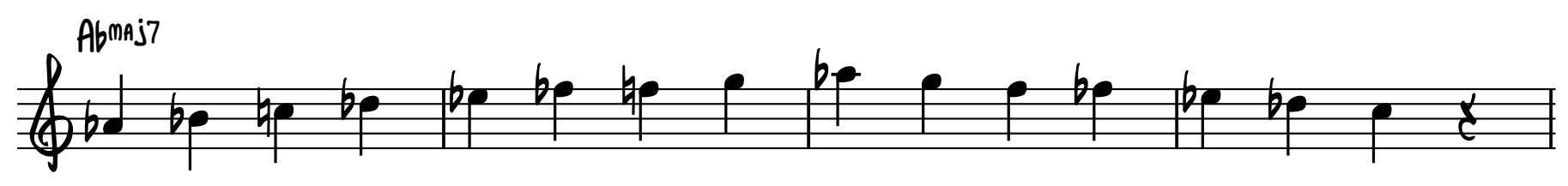

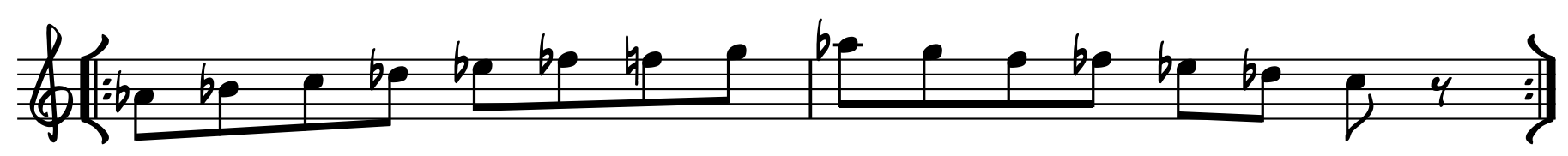

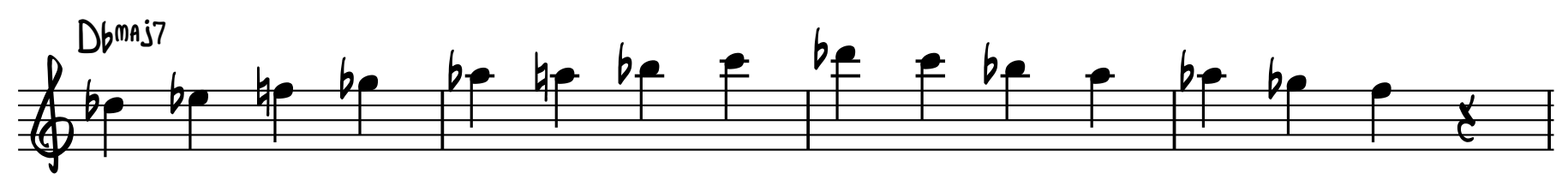

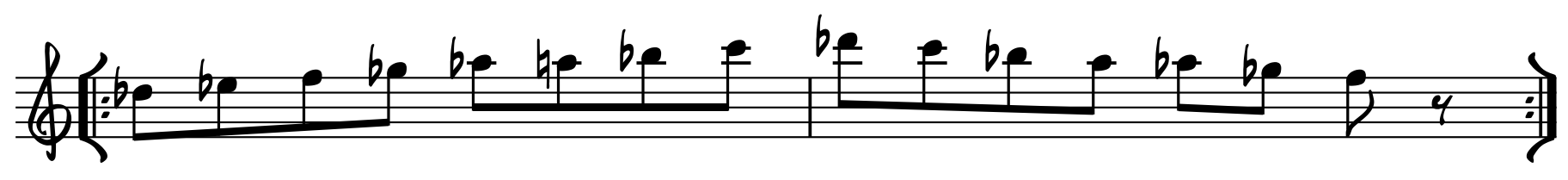

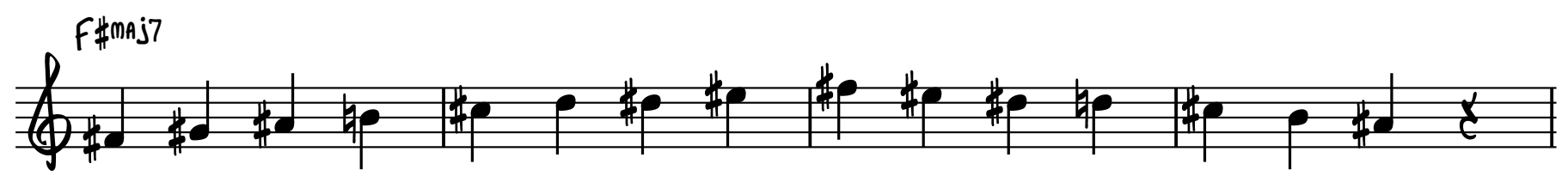



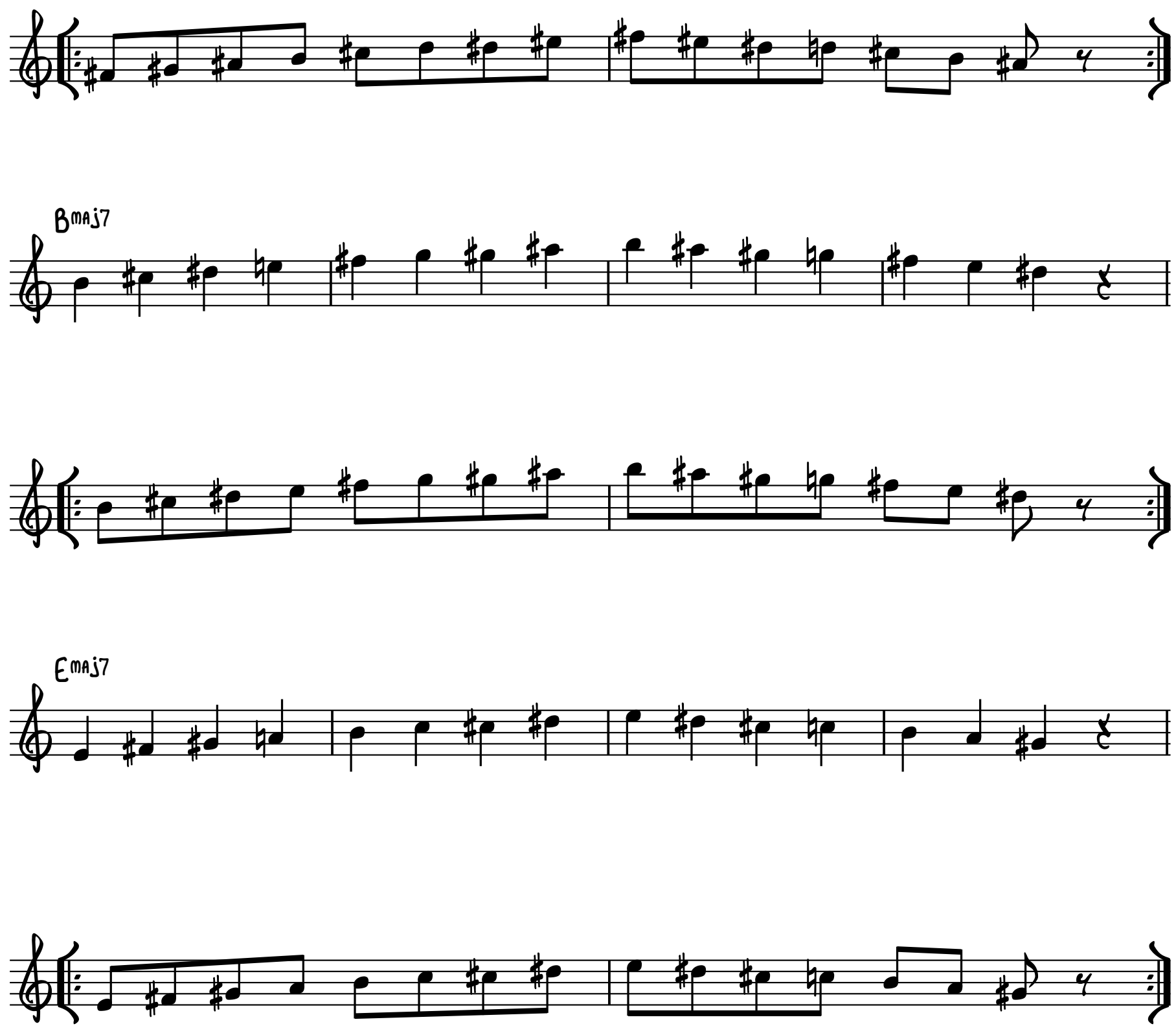

ONCE YOU HAVE MASTERED THIS AT THE FASTEST TEMPO, TRY TO PRACTICE THESE SCALES STARTING ON THE 3RD, 5TH AND 6TH SCALE DEGREES.

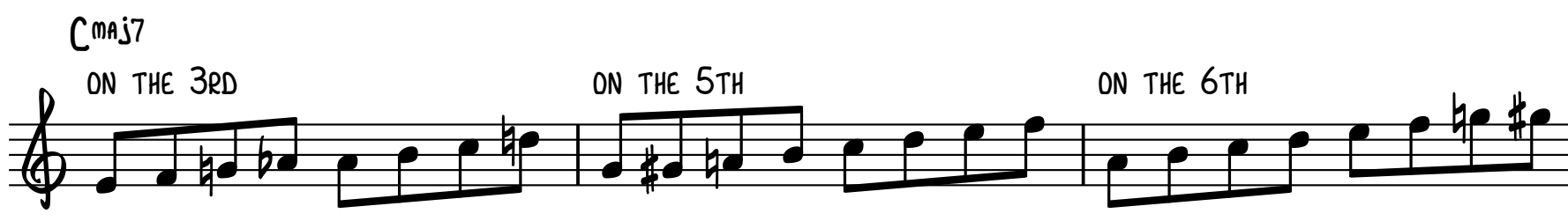

32 


\section{SCALE EXERCISE 2 - DOMINANT BEBOP SCALES}

TRACK 10 - 100 BPM TRACK $11-120$ BPM

$\overbrace{}^{A^{7}}$

PLAY ALL SUCCESSIVE 8TH NOTES WITH

thiS ARTICULATION (NOTICE THE MARRING "SIMLLE")

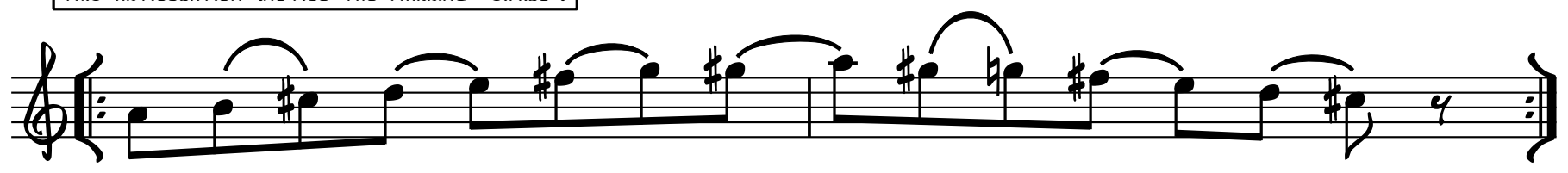

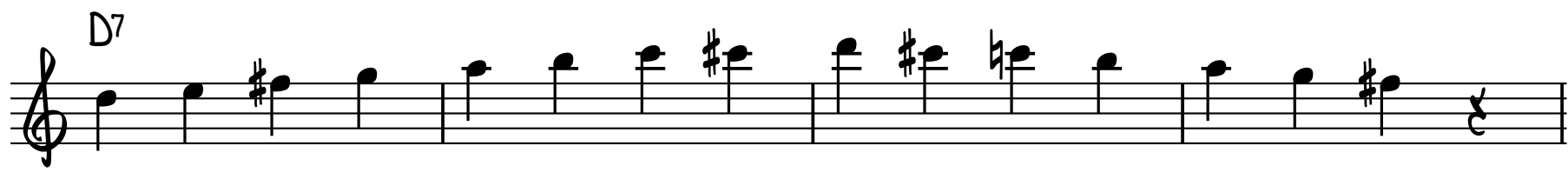

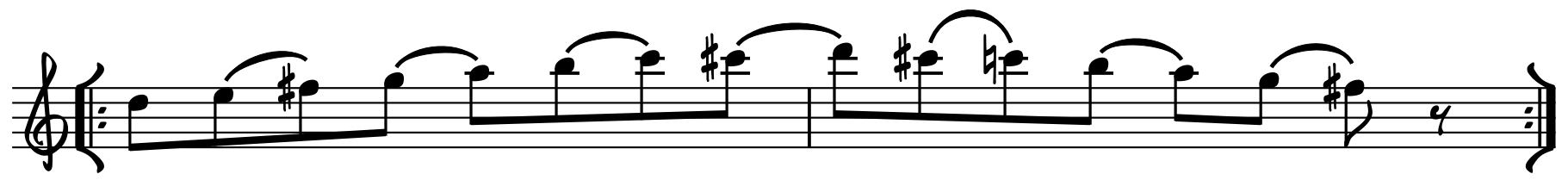

G7

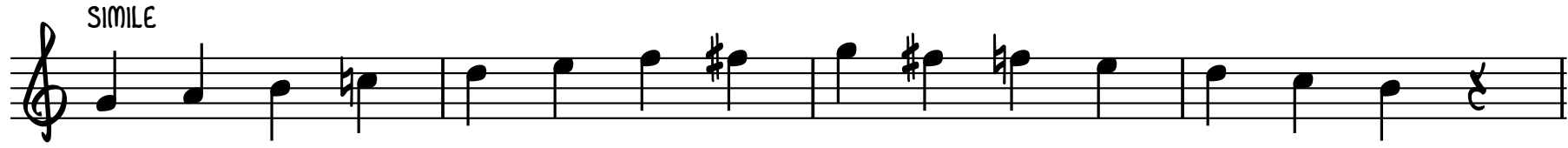

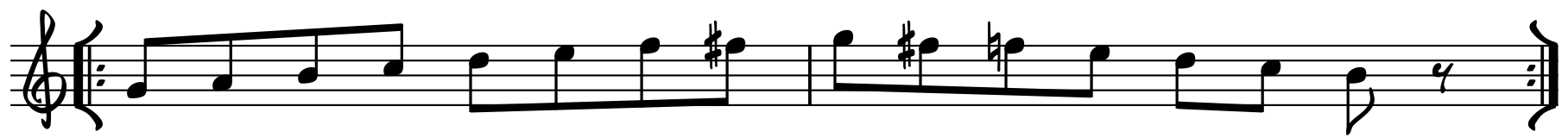

C7

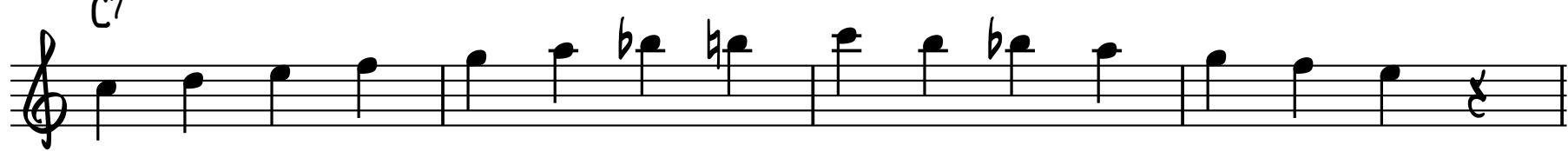

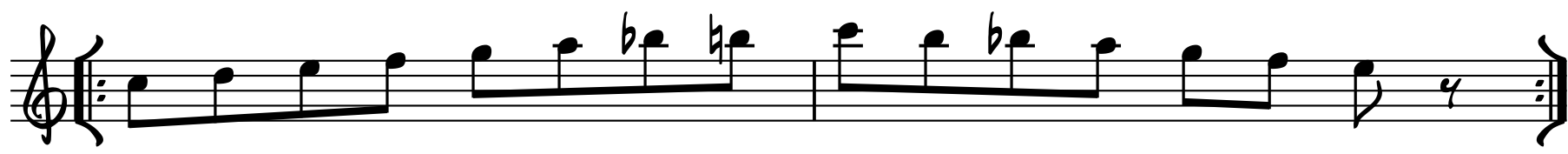

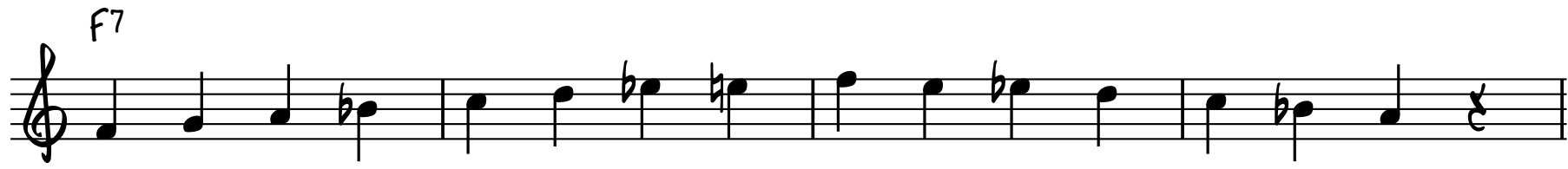




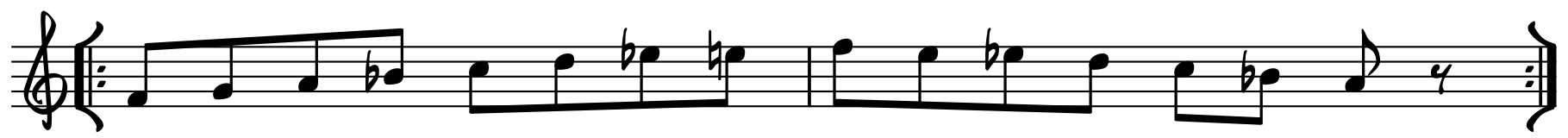

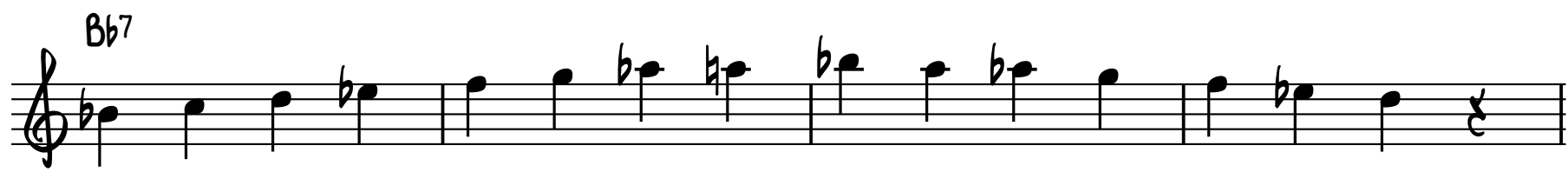

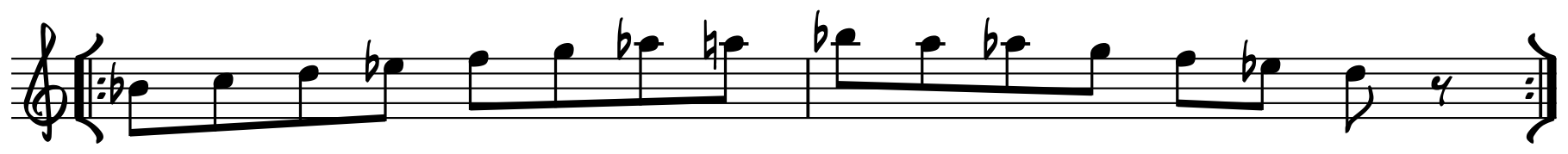

6.

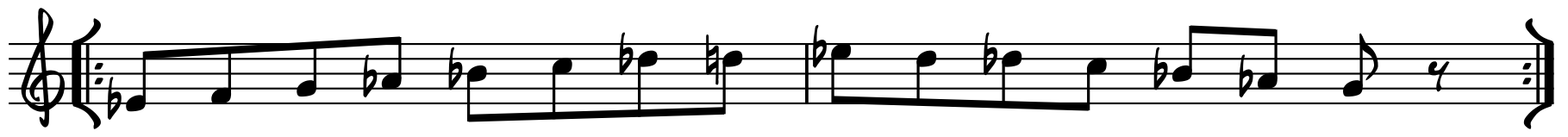

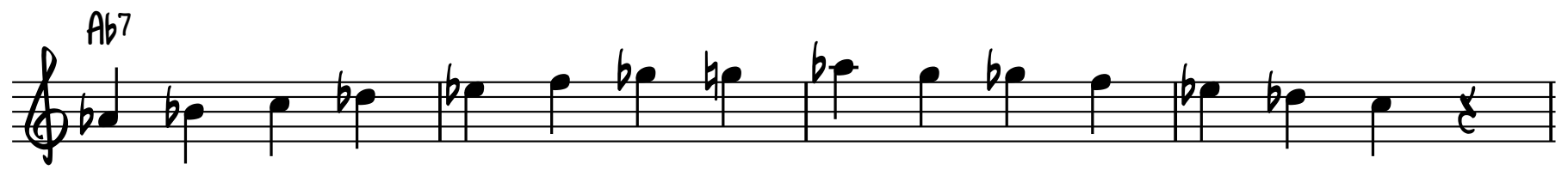

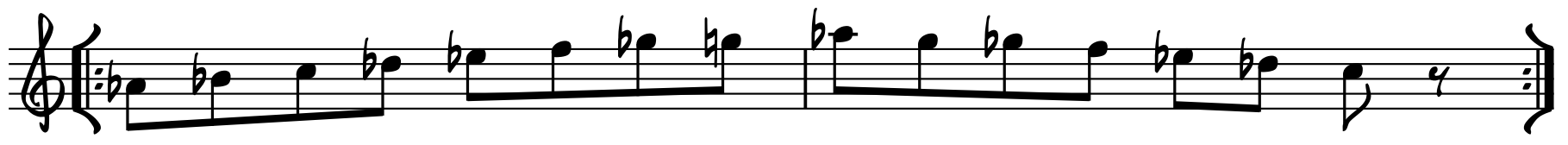

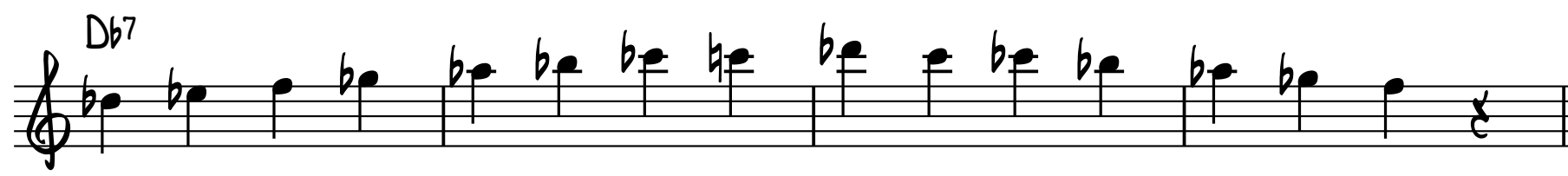

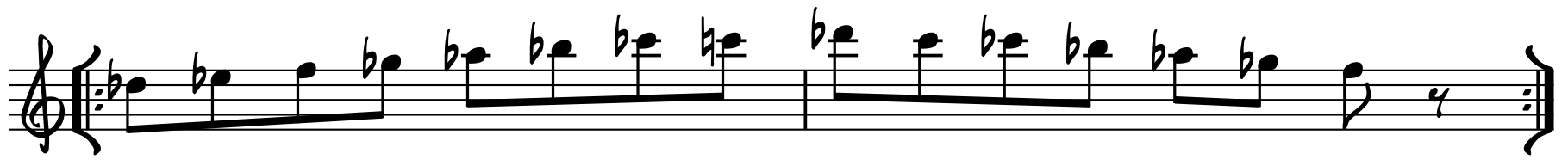

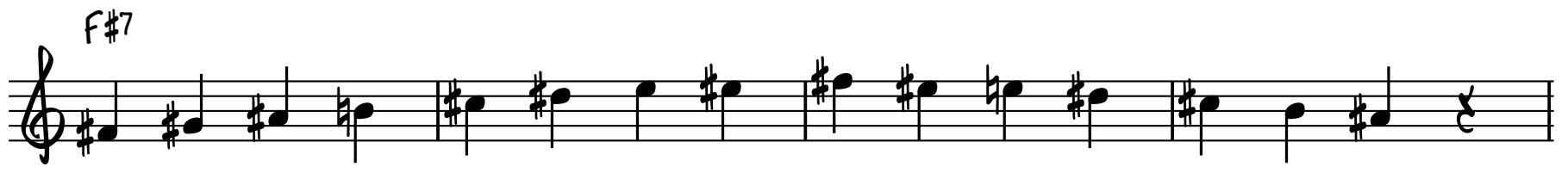



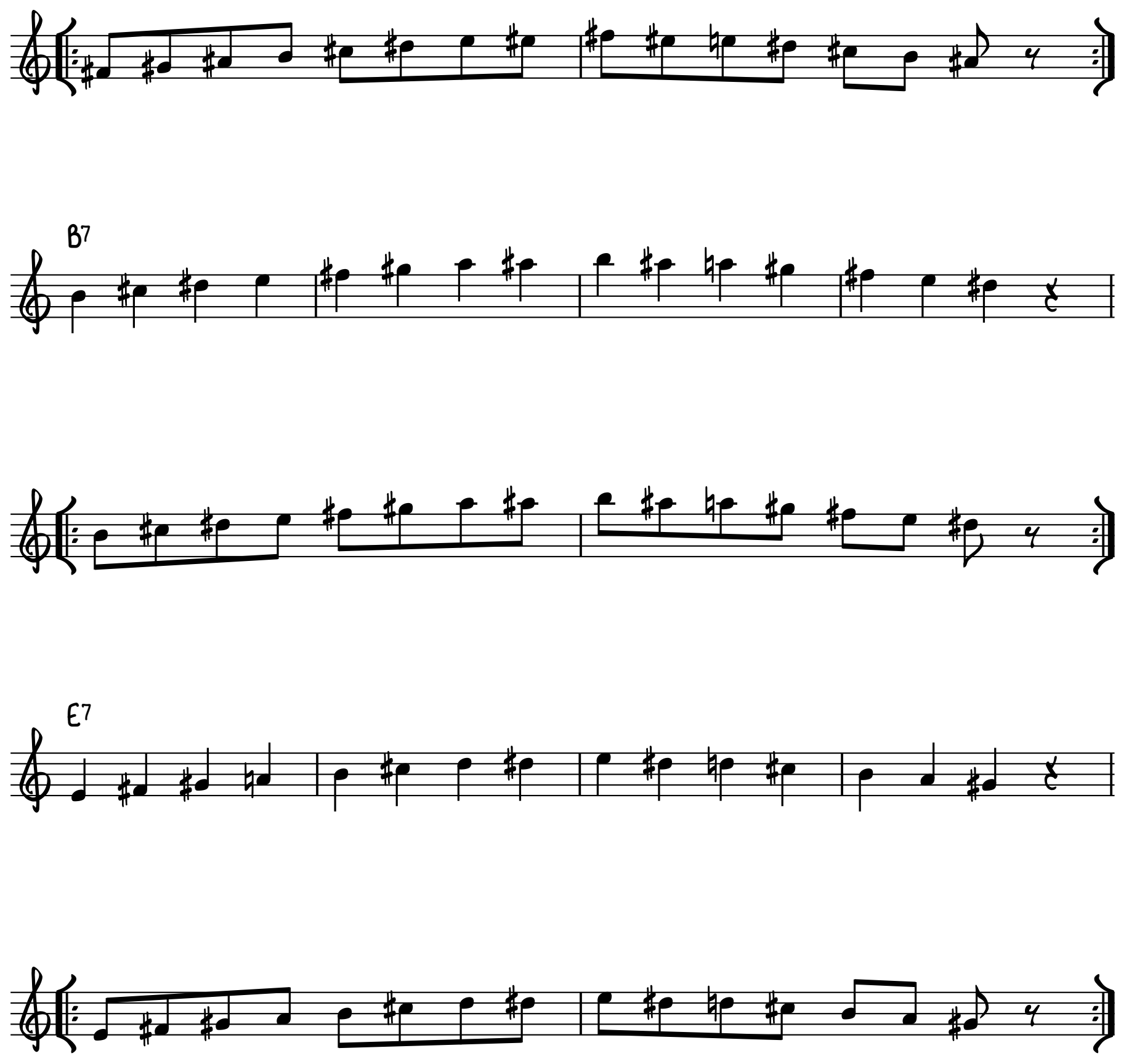

ONCE YOU HAVE MASTERED THIS AT THE FASTEST TEMPO, TRY TO PRACTICE THESE SCALES STARTING ON THE 3RD, 5TH AND FLAT TH SCALE DEGREES.

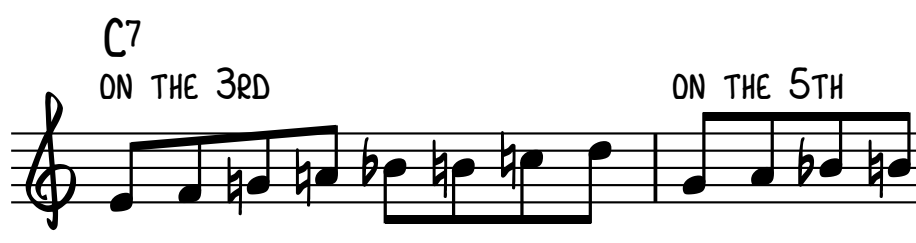

ON THE TH

35 


\section{SCALE EXERCISL 3 - MINOR BEBOP SCALES}

TRACK $19-112$ BPM TRACK $20-132 \mathrm{BPM}$ TRACK $21-152$ BPM

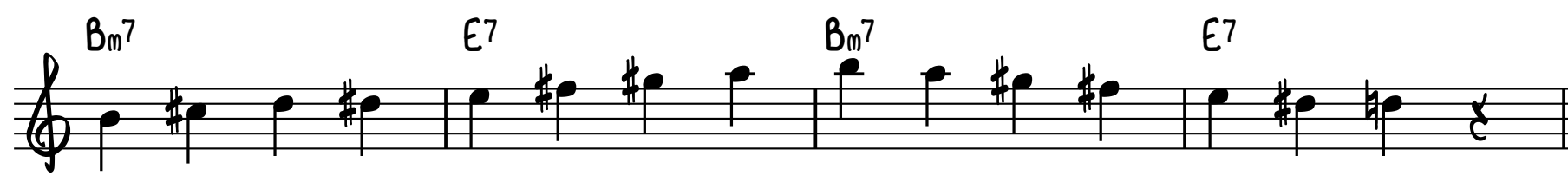

\footnotetext{
PLAY ALL SUCCESSIVE 8TH NOTES WITH

this articulation (nOTICE the marking "simile")
}
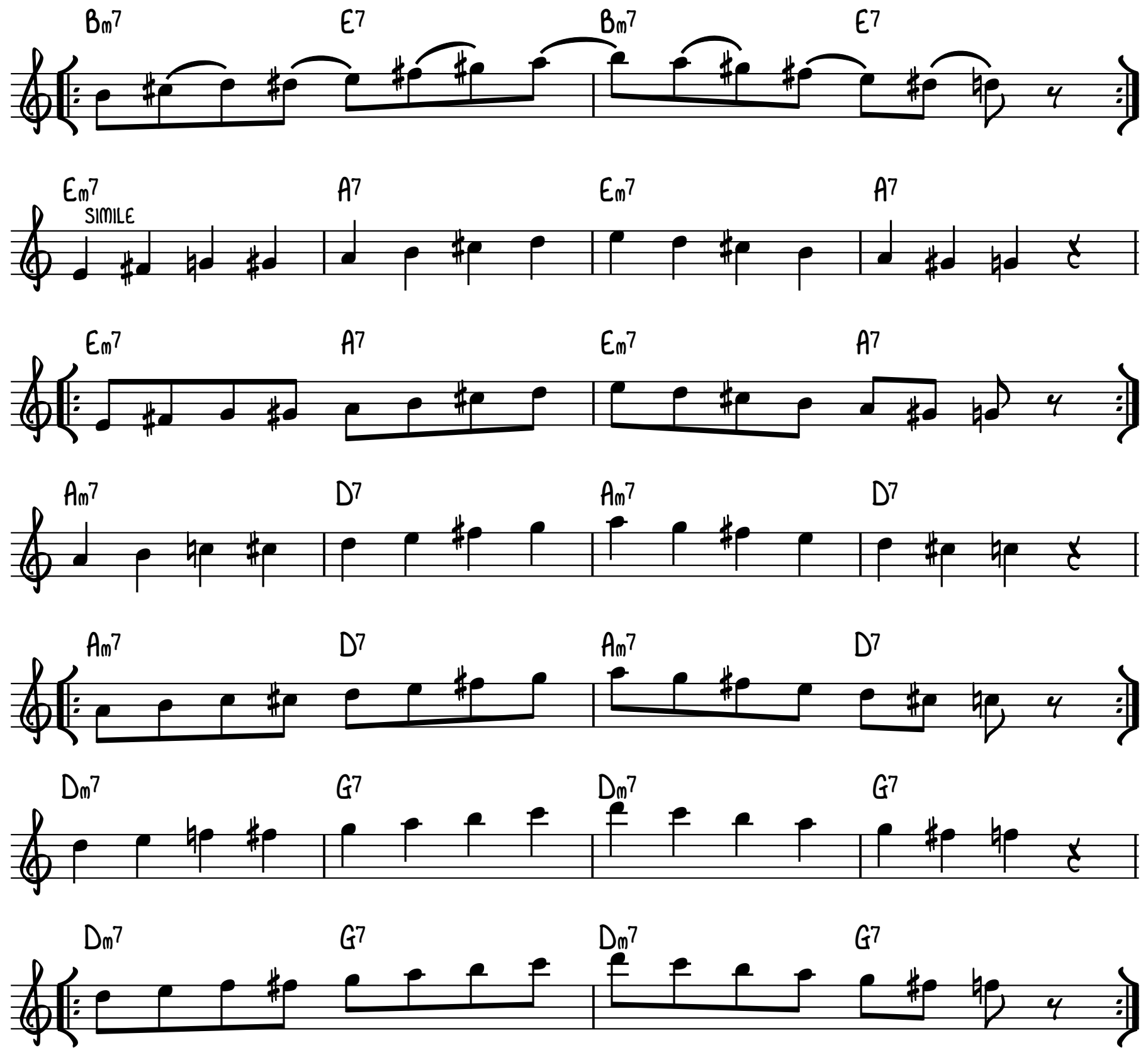
$\mathrm{G}^{\mathrm{Gm} 7}$

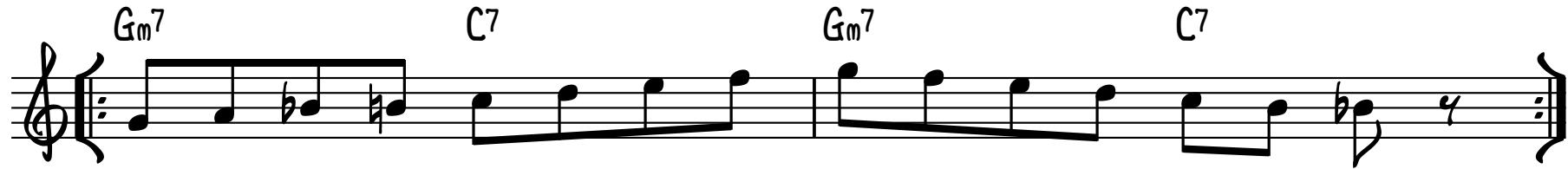

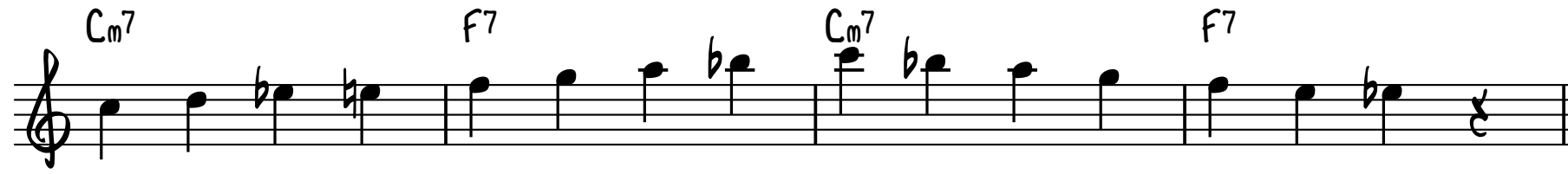

C... be

C.

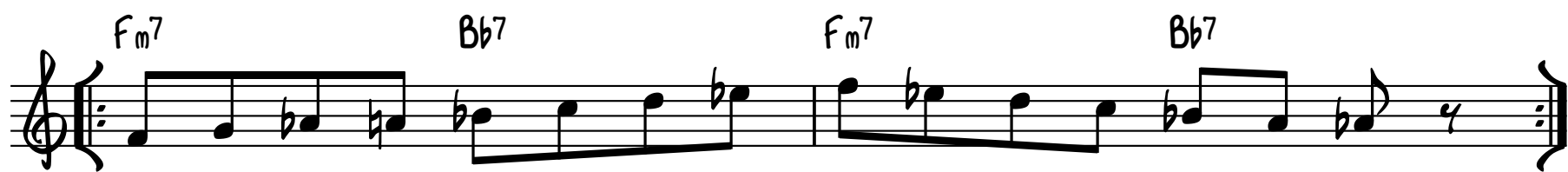
$\mathrm{Bbm}^{7}$
Eb7
$\mathrm{Bbm}^{7}$
Eb7

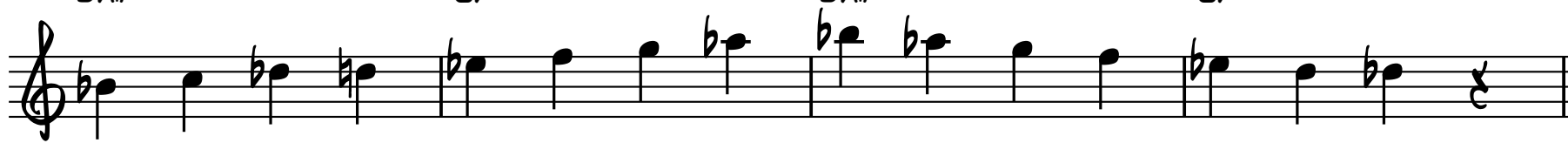

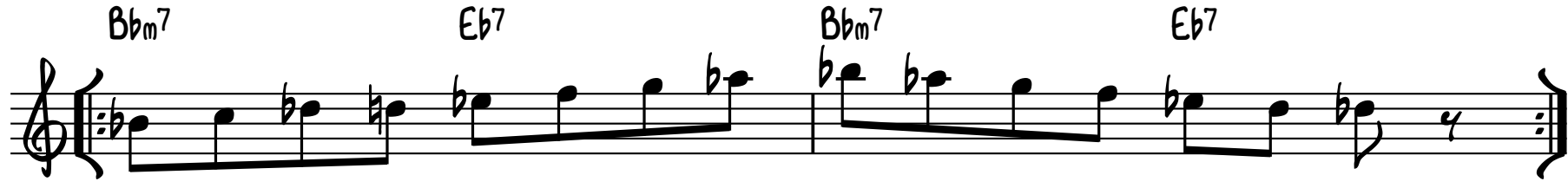

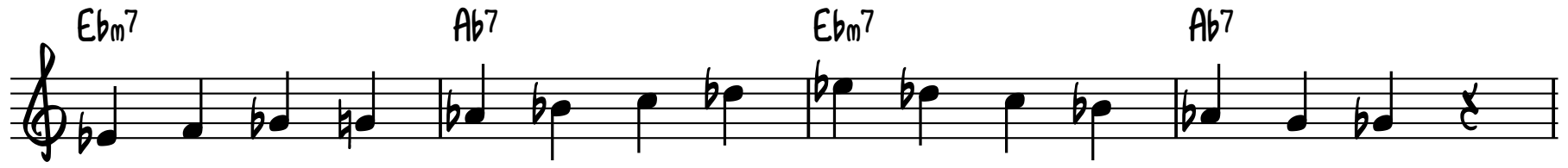

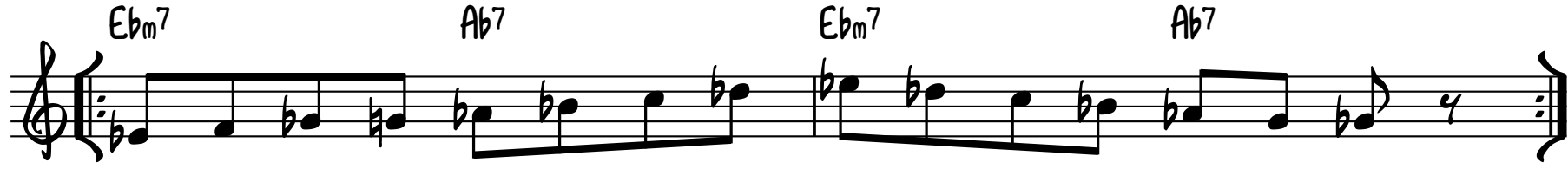


$f_{0}^{\mathrm{Abm} \mathrm{m}^{7}}$
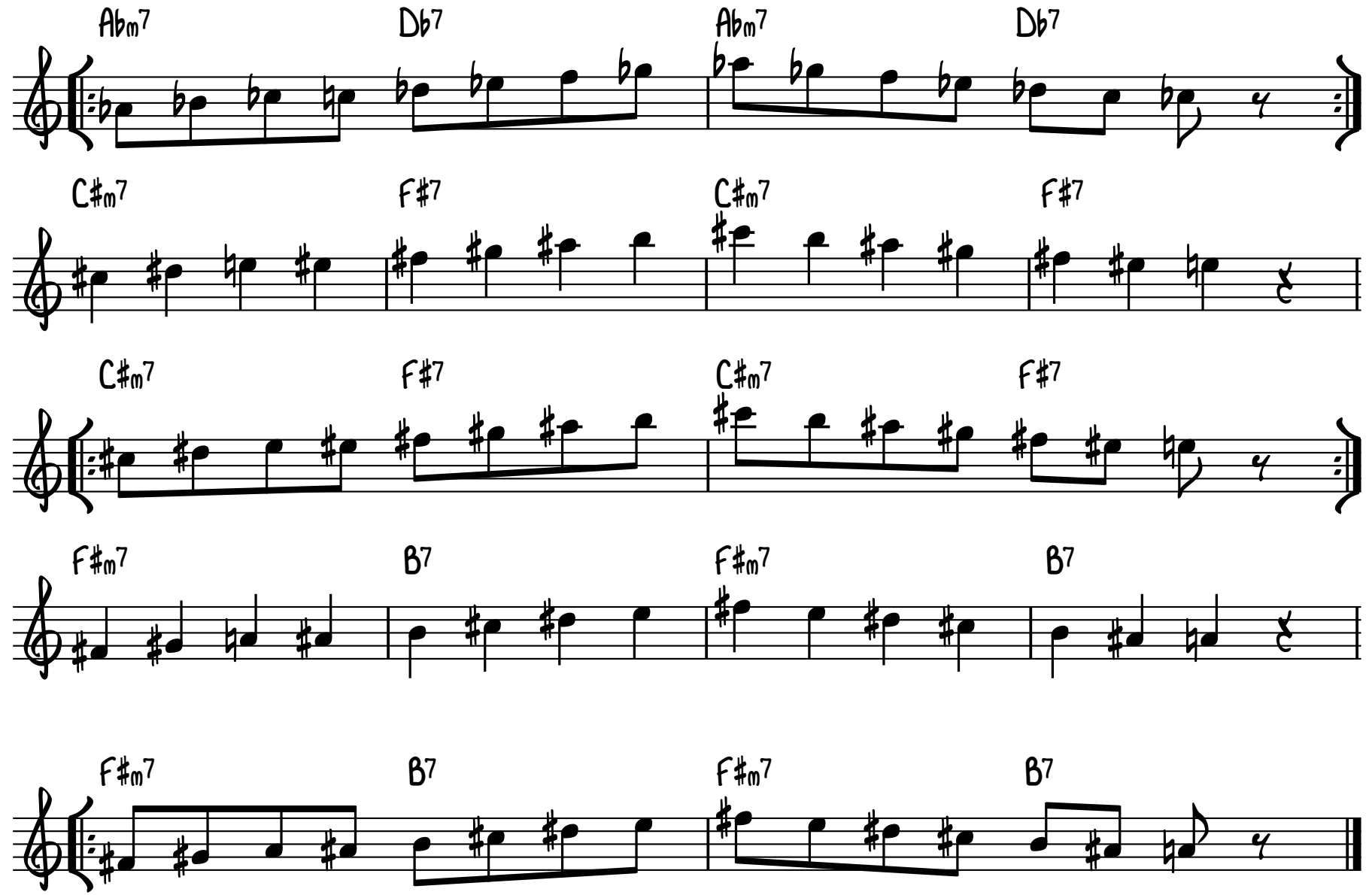

THIS IS THE 5TH MODE OF THE DOMINANT BEBOP SCALE. FOR FURTHER EXPLORATION, REFER tO THE EXPANSION IN SCALE EXERCISE 2. 


\section{ONE BAR TWO FIVE}

SWING

TRACK 22 - 100 BPM

TRACK 23 - 120 BPM

Gm7 C7 Fmaj7 $\quad F_{m}^{7} \quad$ Bb7 EbMAj7 TRACK 24-140 BPM

$\# 4$ (4)

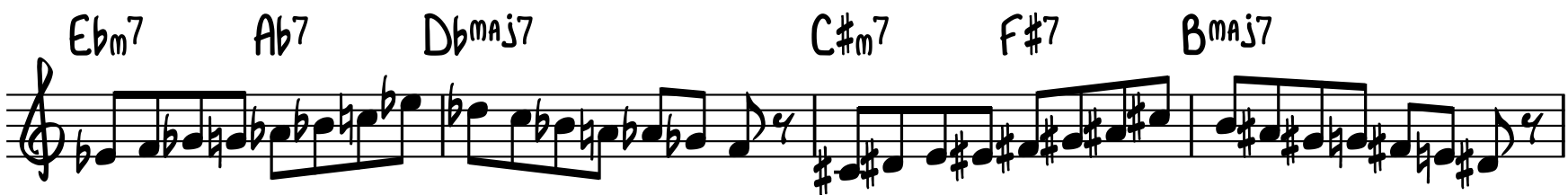

$\mathrm{B}^{\mathrm{Bm}^{7}} \mathrm{E}^{\mathrm{Amaj7}} \mathrm{Am}^{\mathrm{m}} \mathrm{D}^{7}$

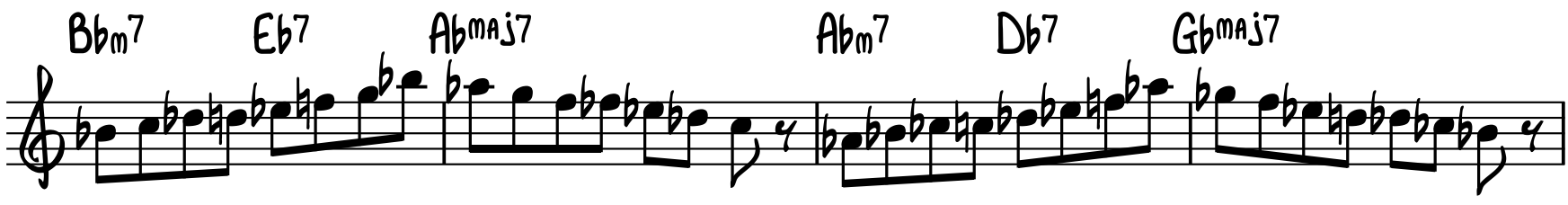

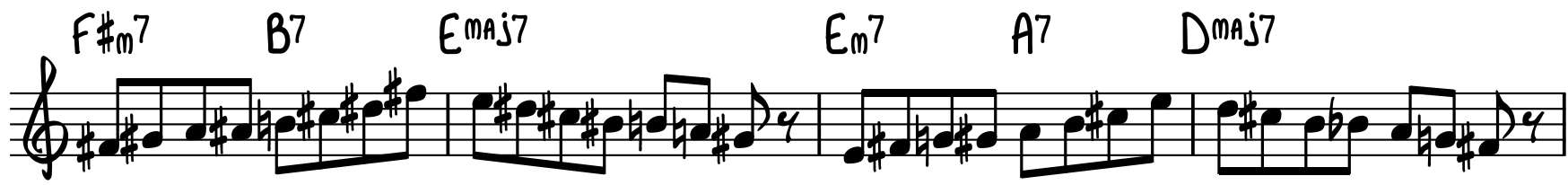

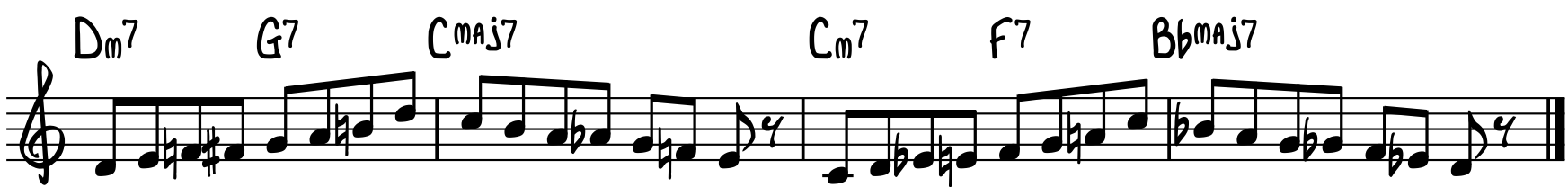




\section{TWO BAR II-V}

$\overbrace{}^{\mathrm{Am}^{7}} \mathrm{D}^{7}$

$f^{\mathrm{Gm} 7}{ }^{C 7}$

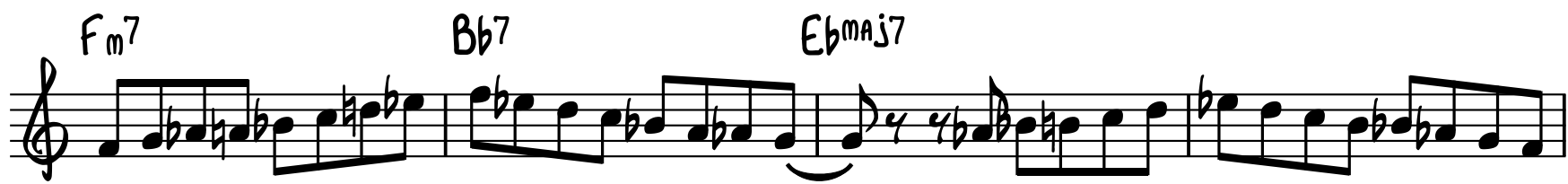

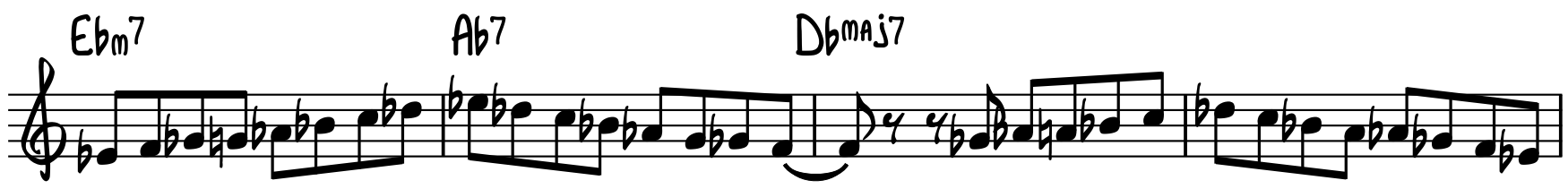

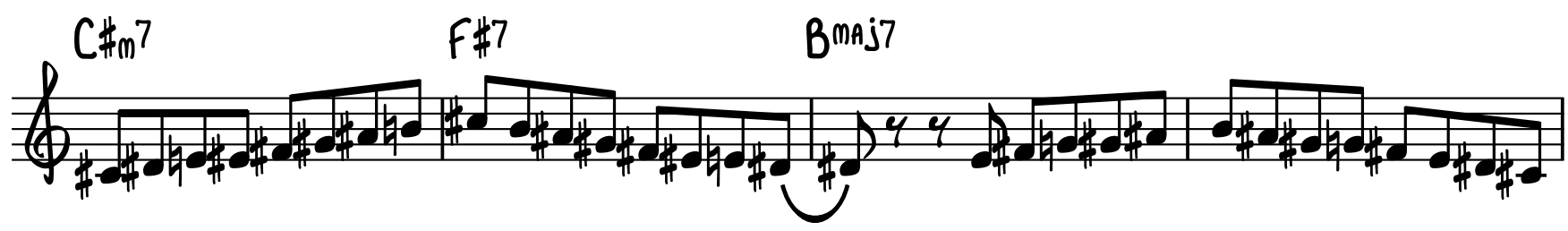

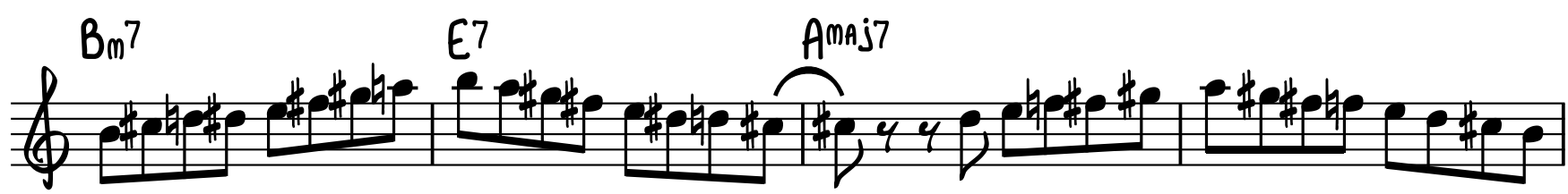

$\mathrm{Cm}_{m} \quad \mathrm{F7} \quad$ Bbmaj7

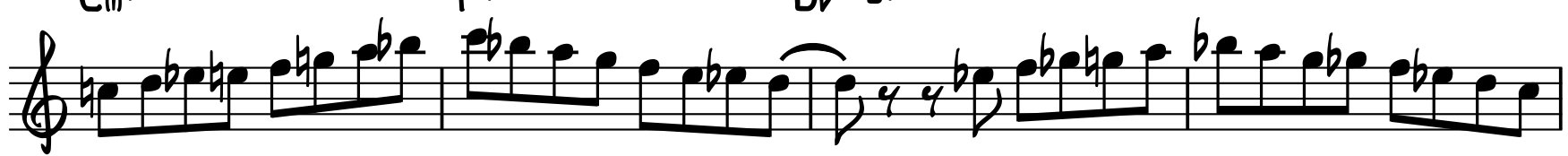




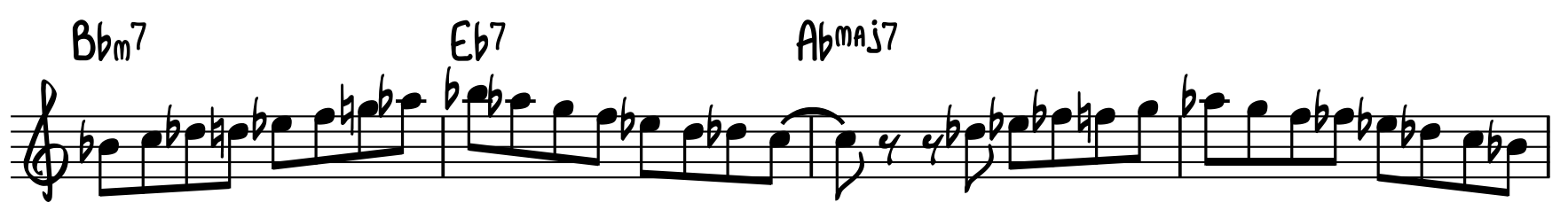

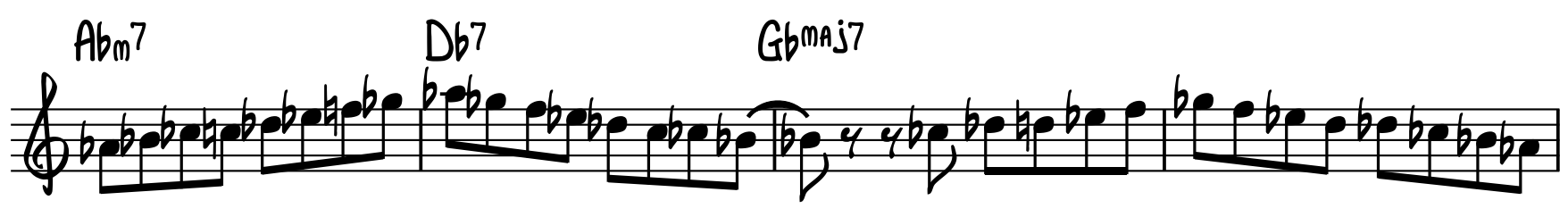

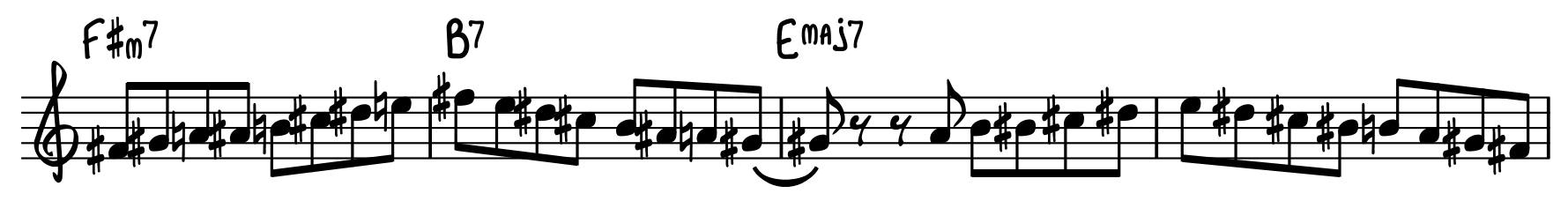

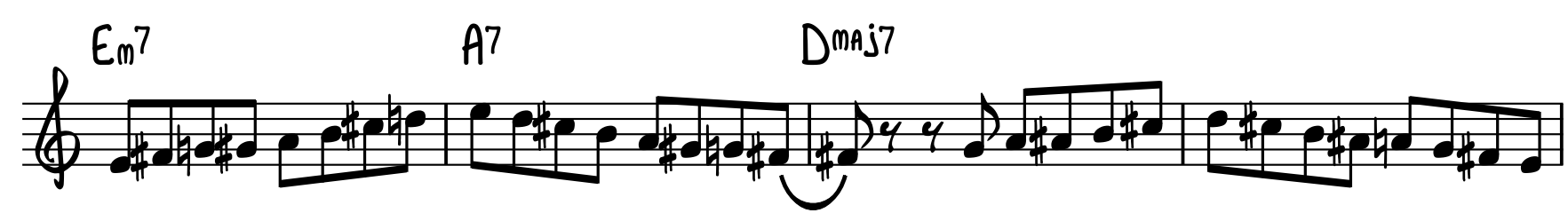

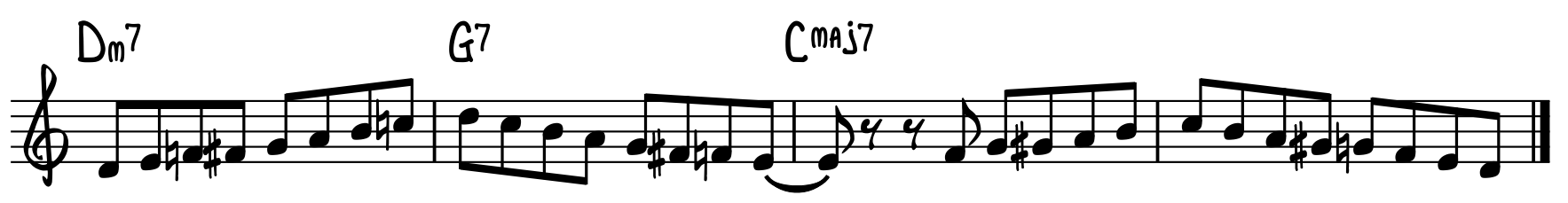


I TO IV MODULATION THROUGH III

TRACK $28-92 \mathrm{BPM}$
TRACK $29-112 \mathrm{BPM}$

TRACK 30 - 132 BPM

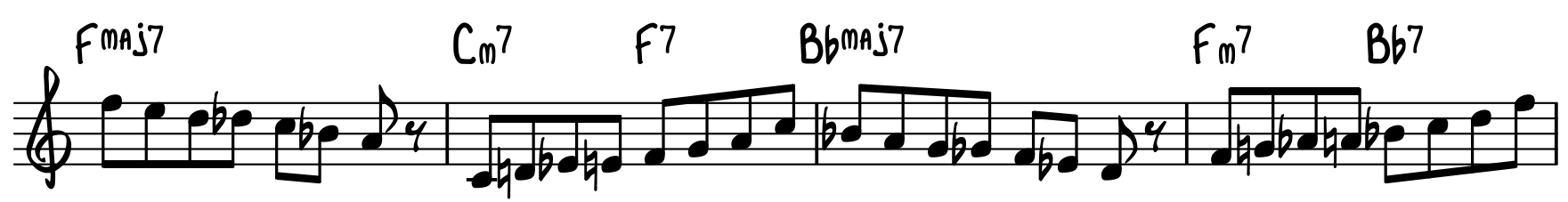

Ebmaj7 Abm $E b 7 \quad A b$ maj $\quad b^{2} \quad A b 7$

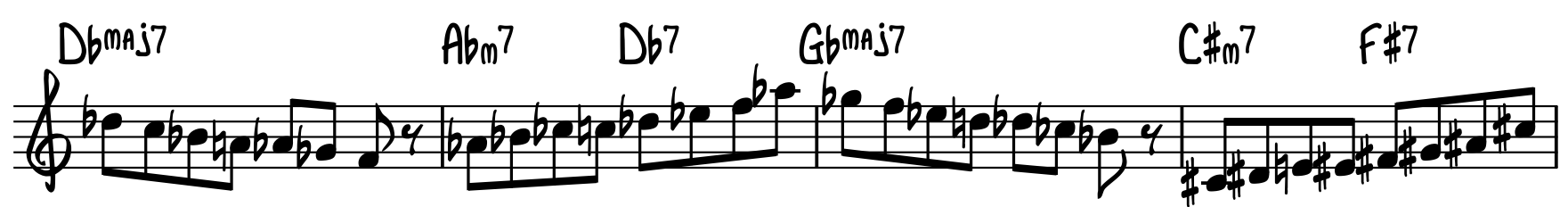

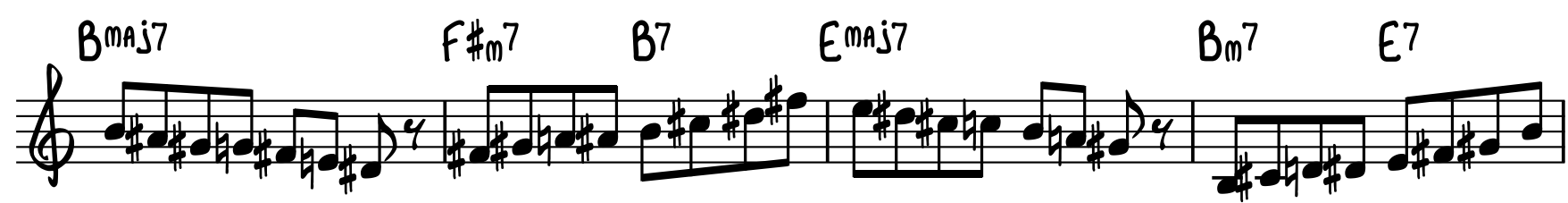

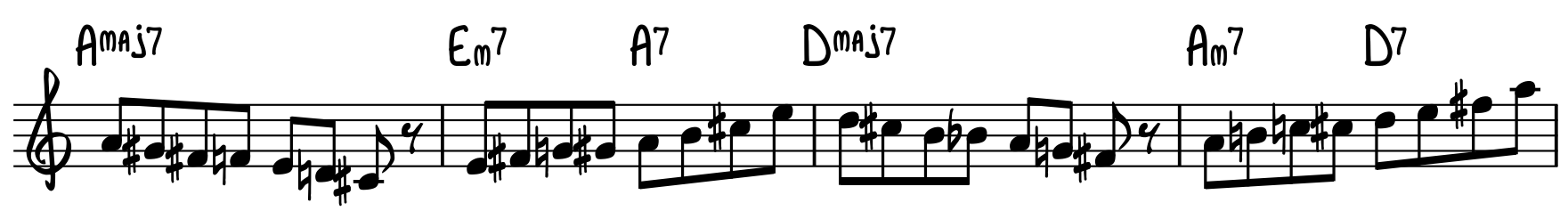

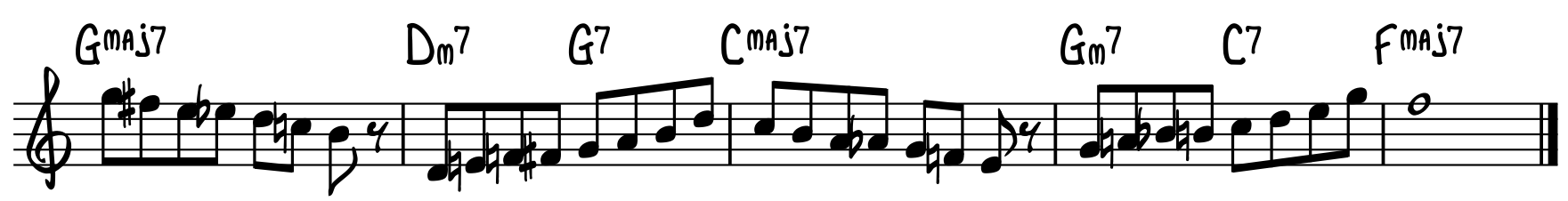


(4) SIMILE

5 D

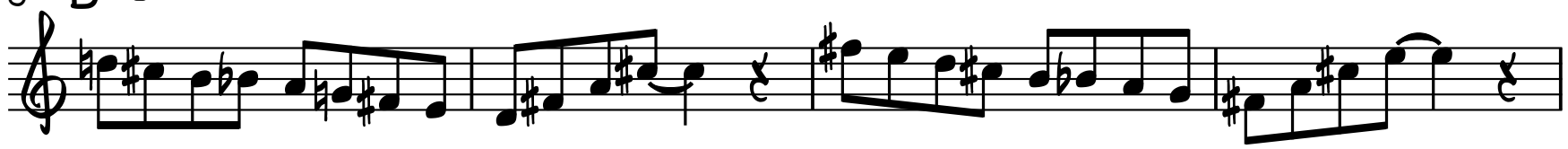

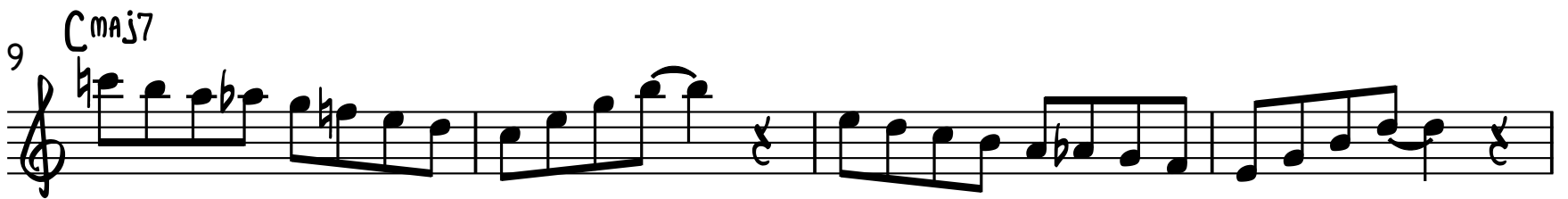

13 Dmaj7

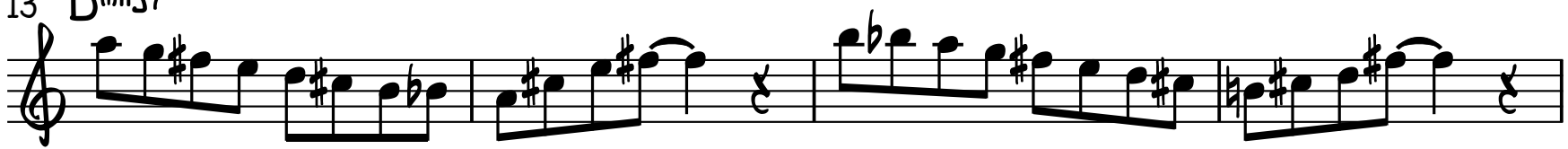

${ }_{17}^{\text {Emaj7 }}$

21 D'maj7 $\quad G^{\text {maj7 }}$

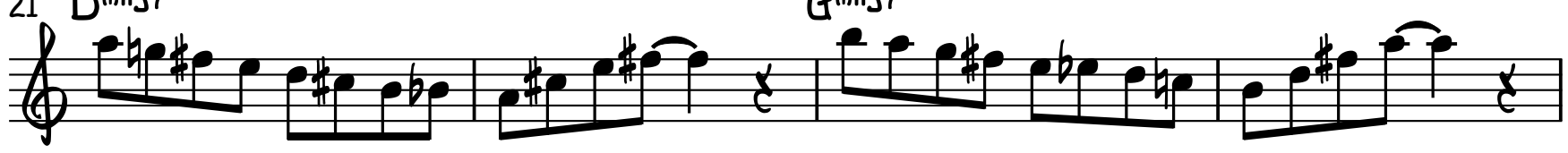

25 Cmaj7

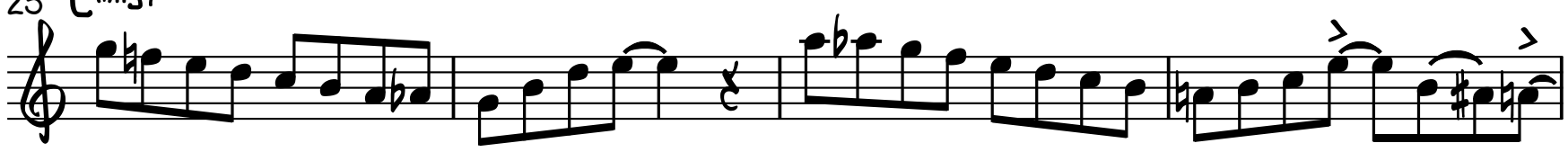

29 D maj7

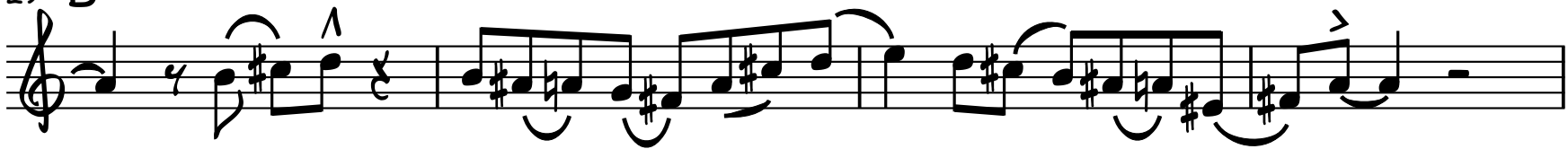


ALTO SAXOPHONE

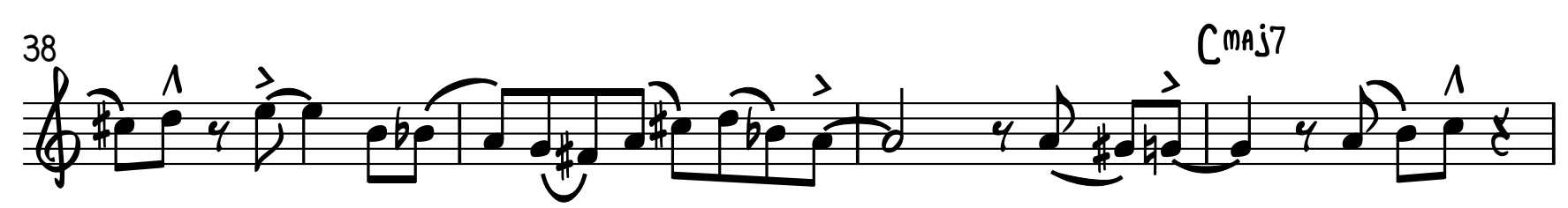

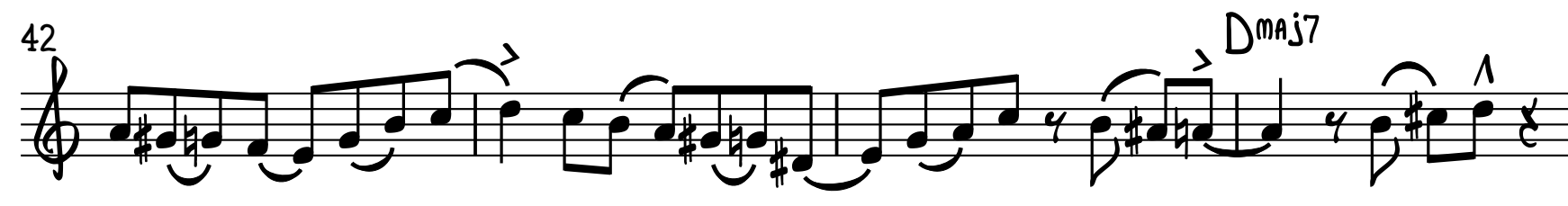

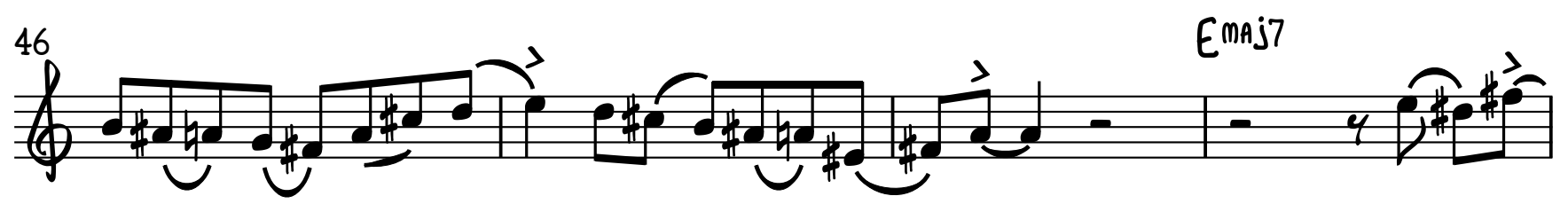

10

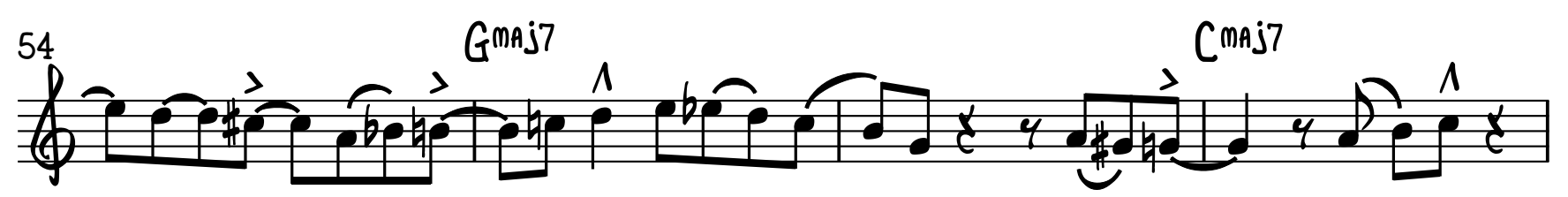

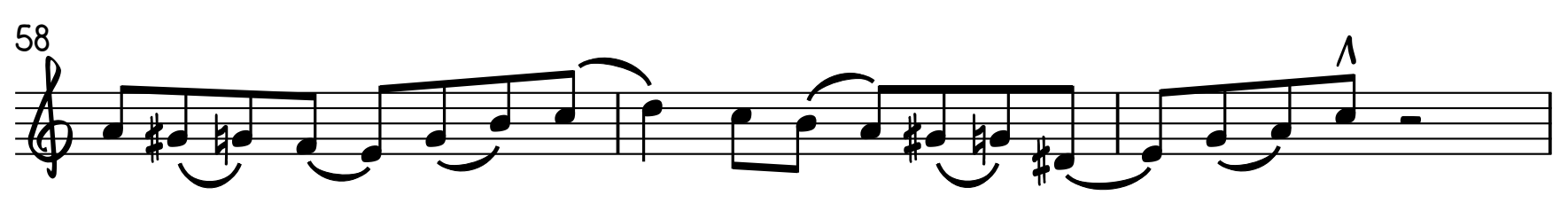

(1) क्या 


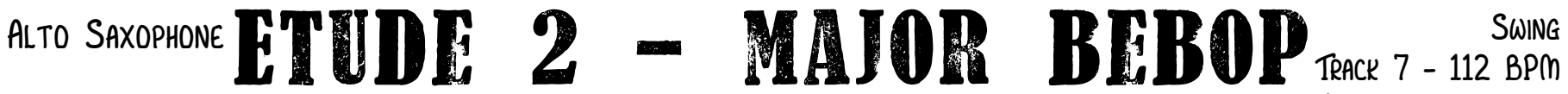

TRACK 8 - 132 BPM

TRACK 9 - 152 BPM
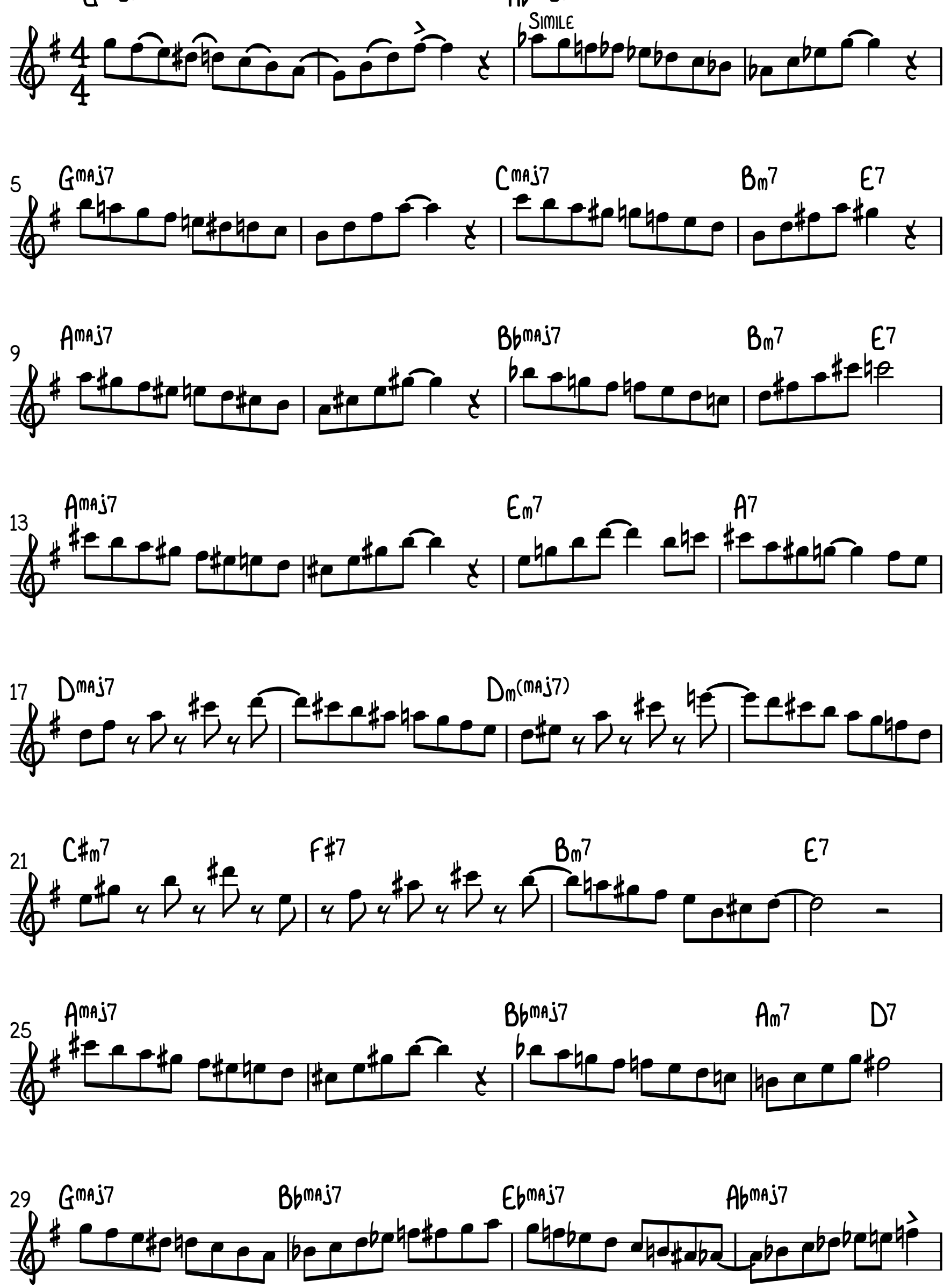

45 
2

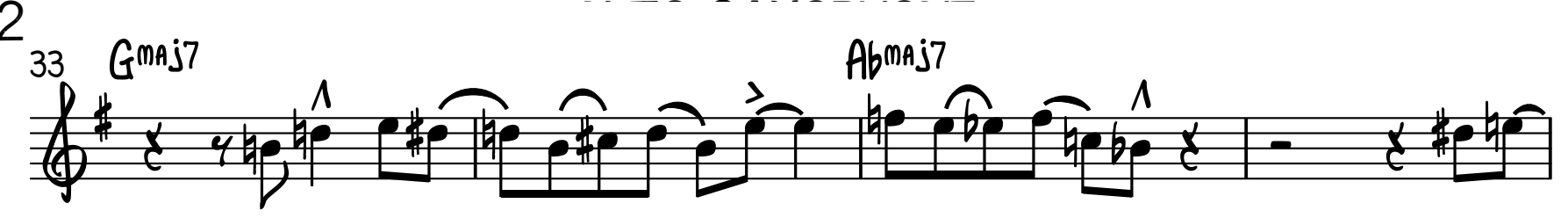

37 Gmij7
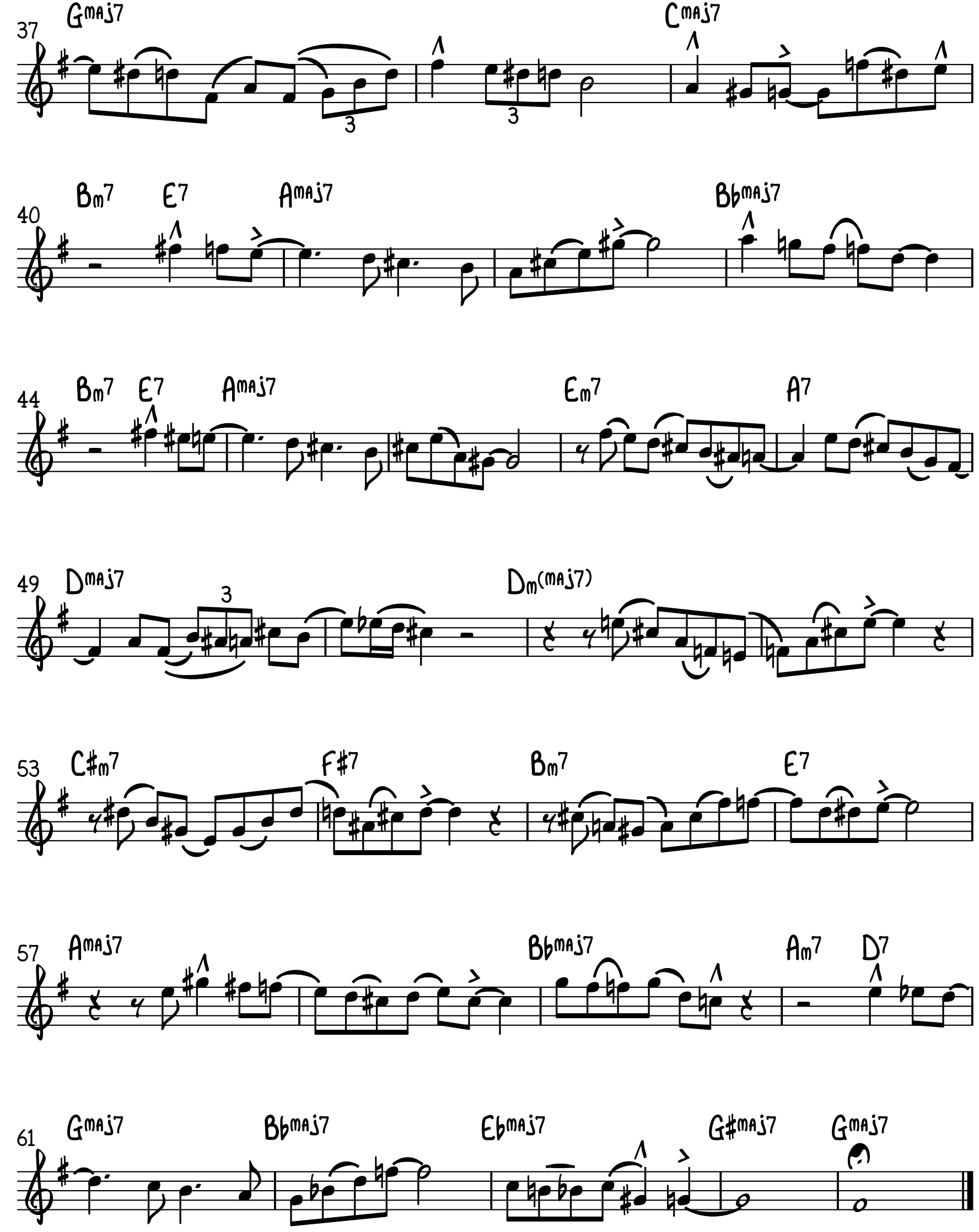
Ero swome

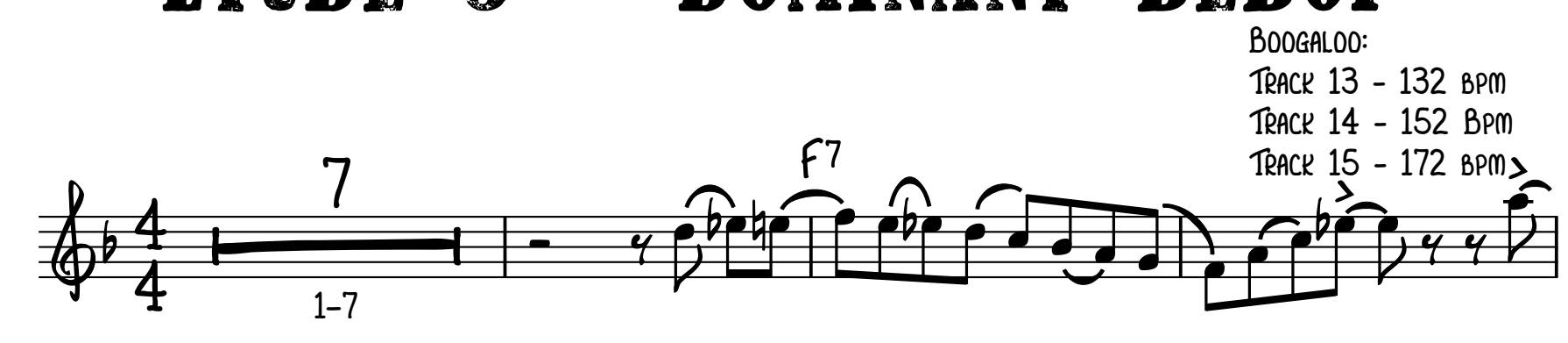

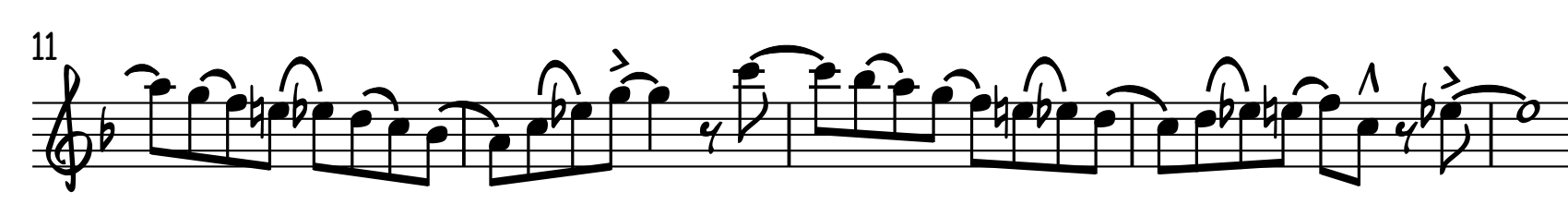

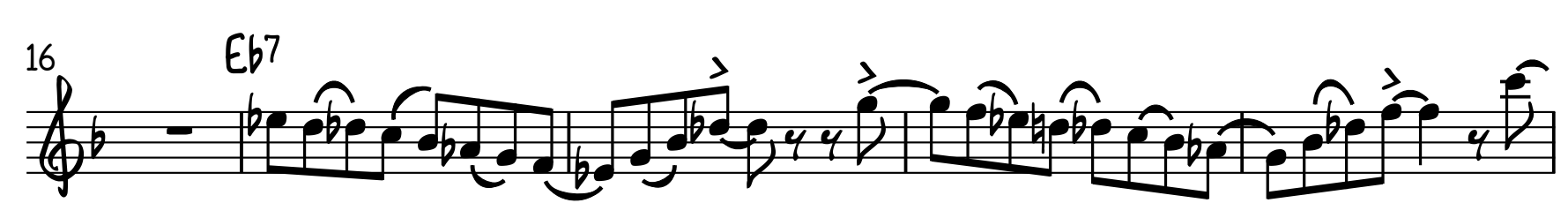

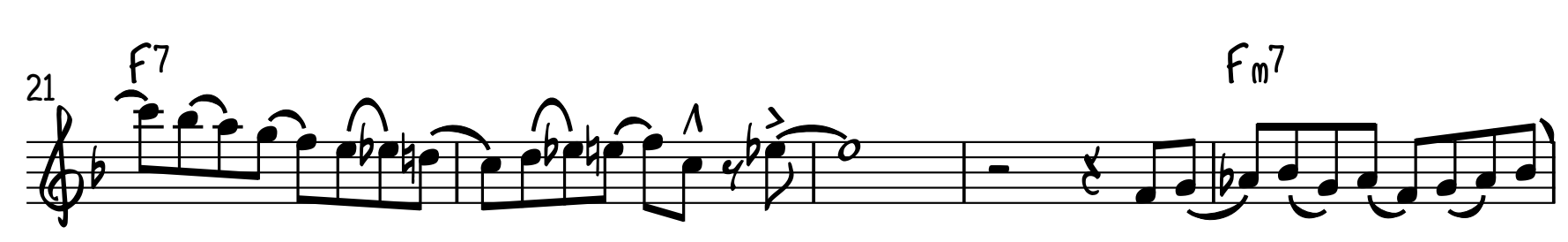

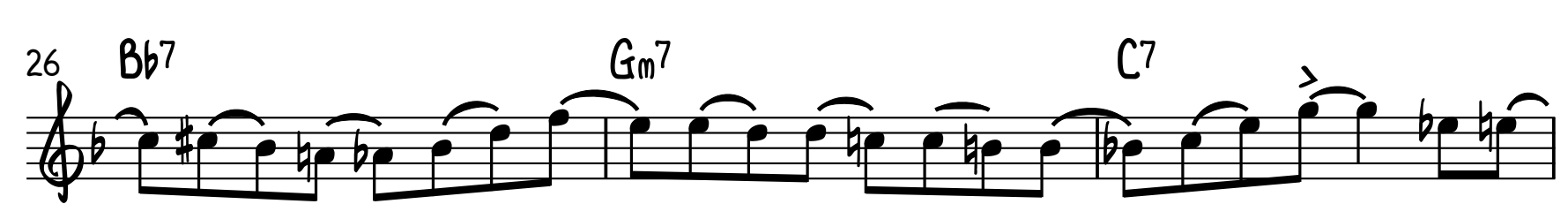

(29) 
2

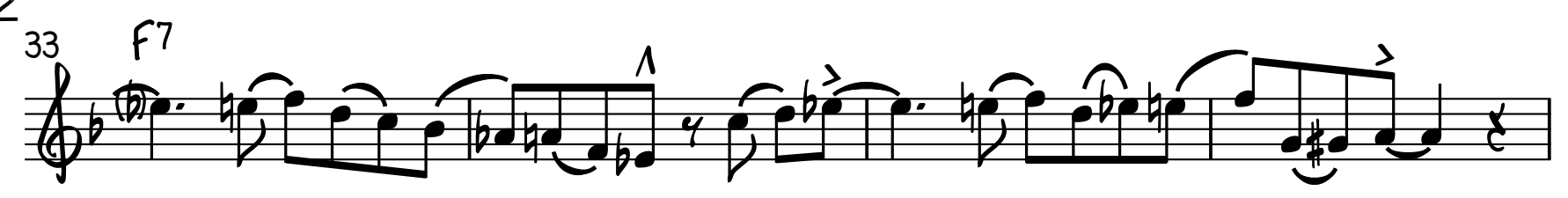

(4t)

$\int^{42}$

$\mathrm{H}^{46}$

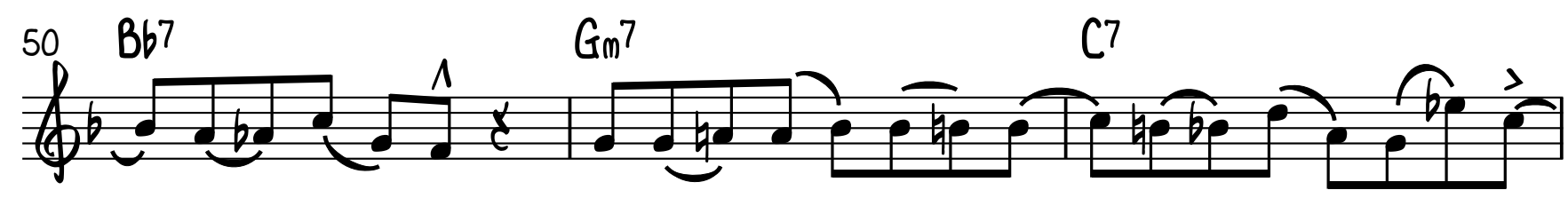

$f^{b-5}{ }^{67}$ 
Alto SAXophone

ETUDE 4 - DOMINANT BEBOP

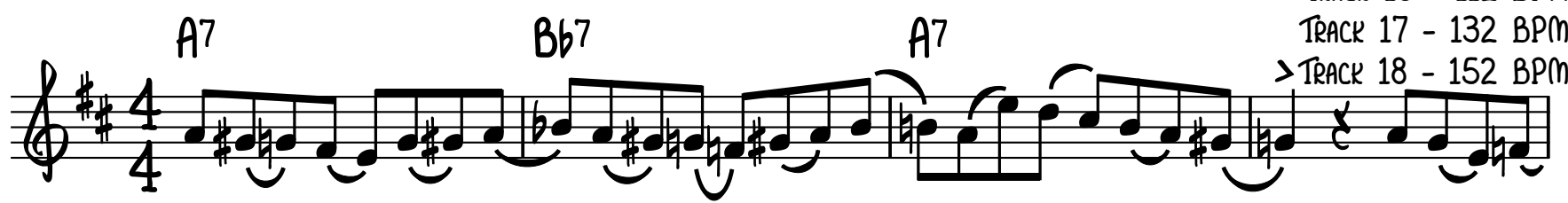

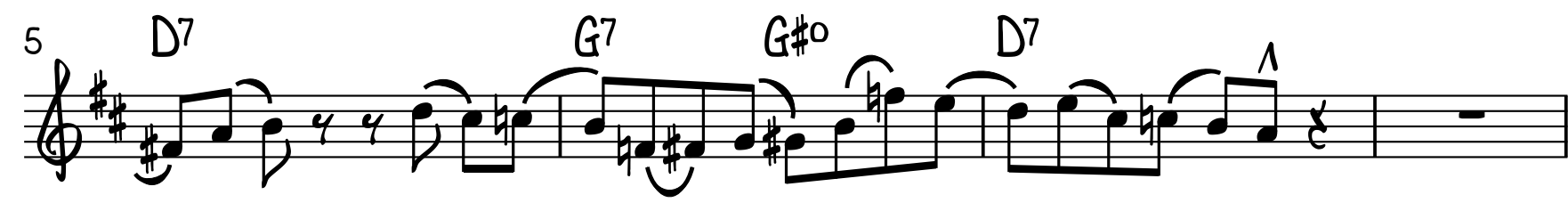

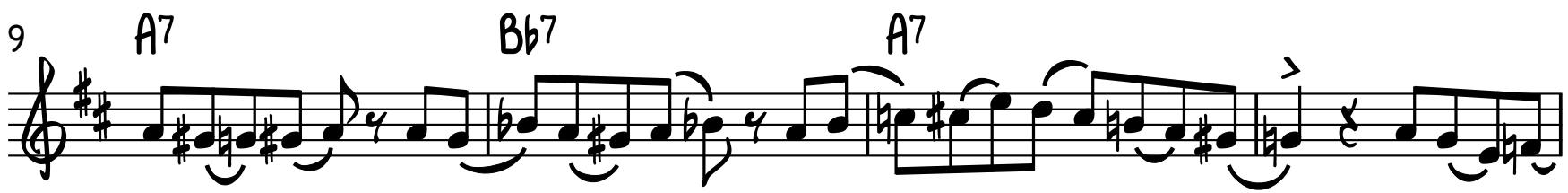

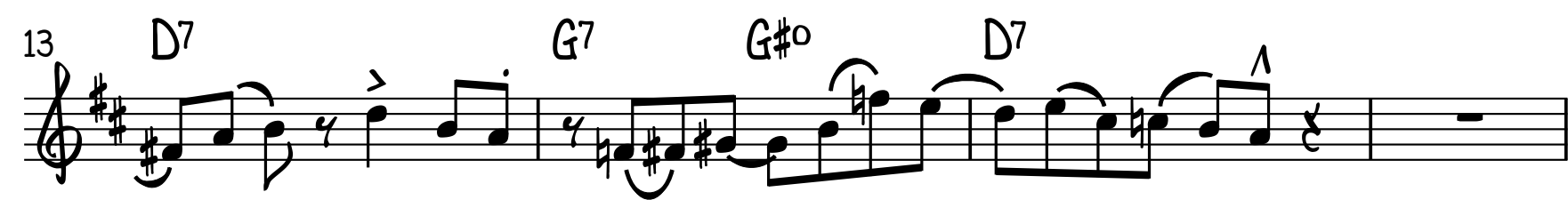

17 4 (17)

21. E7

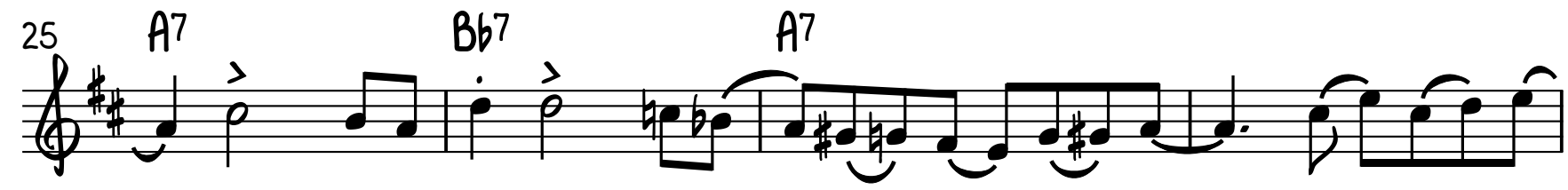

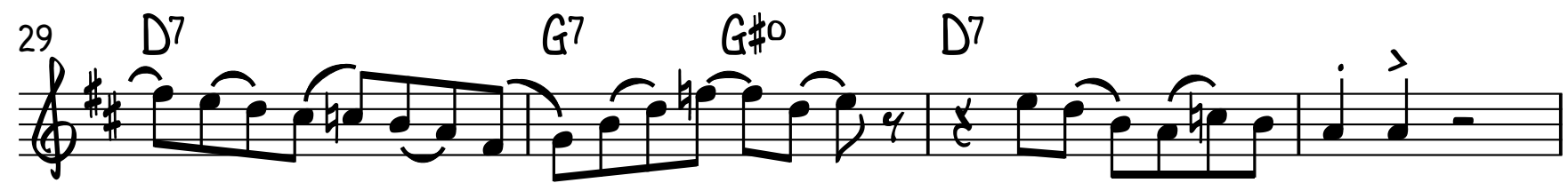


2

ALTO SAXOPHONE

337
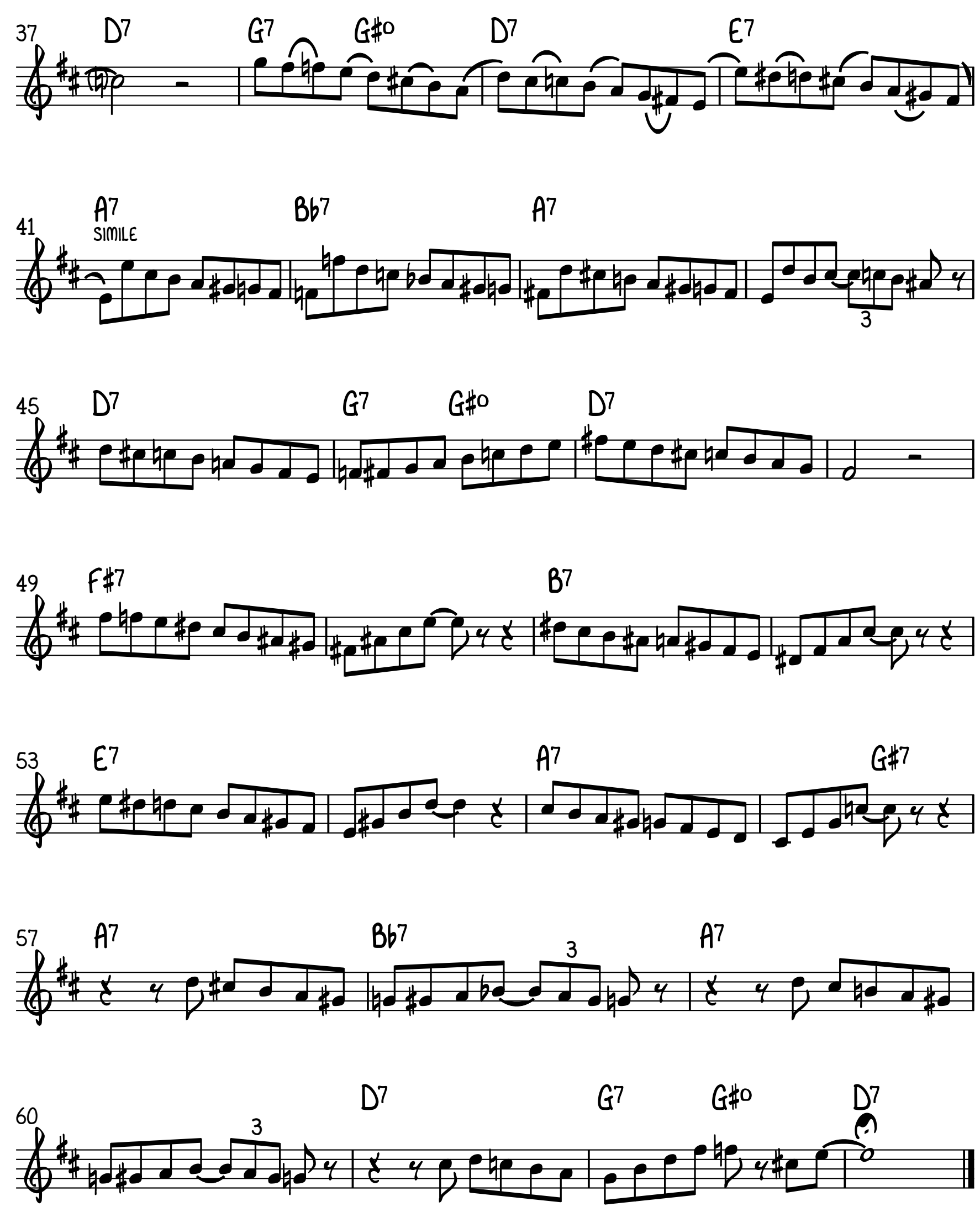
ALTO SAXOPHONE

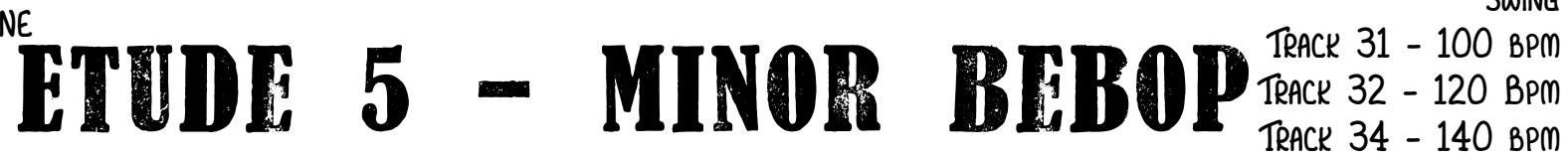
管

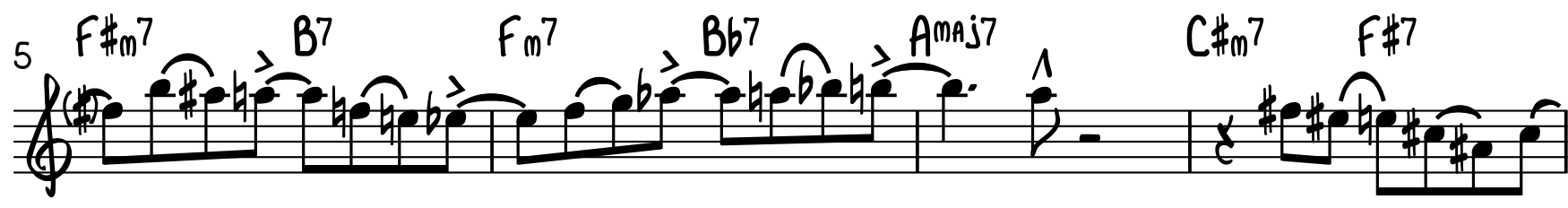

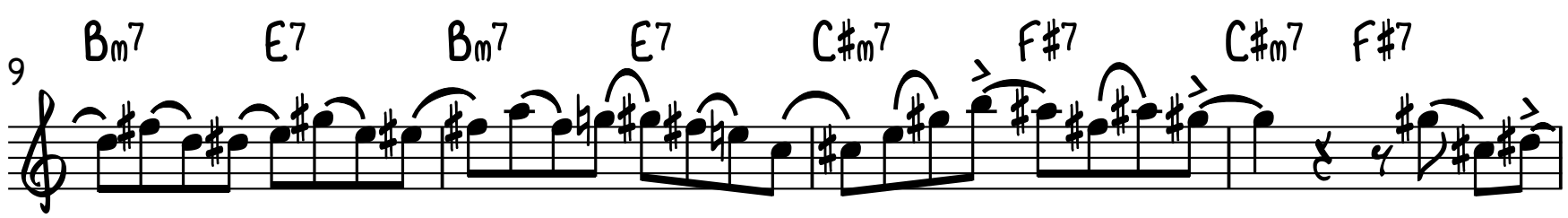
13: Fant

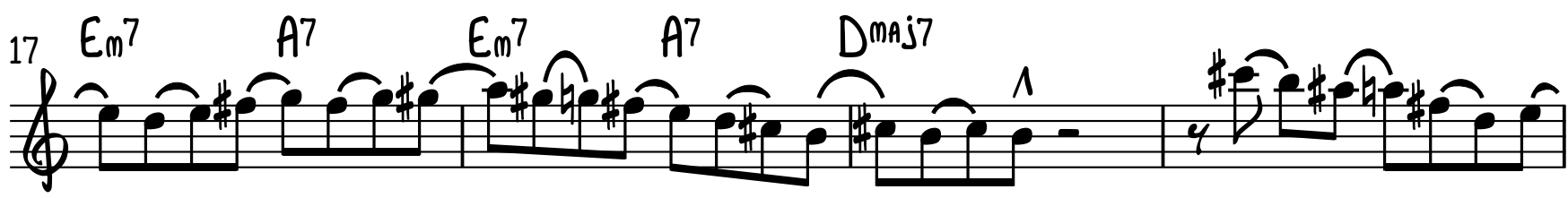

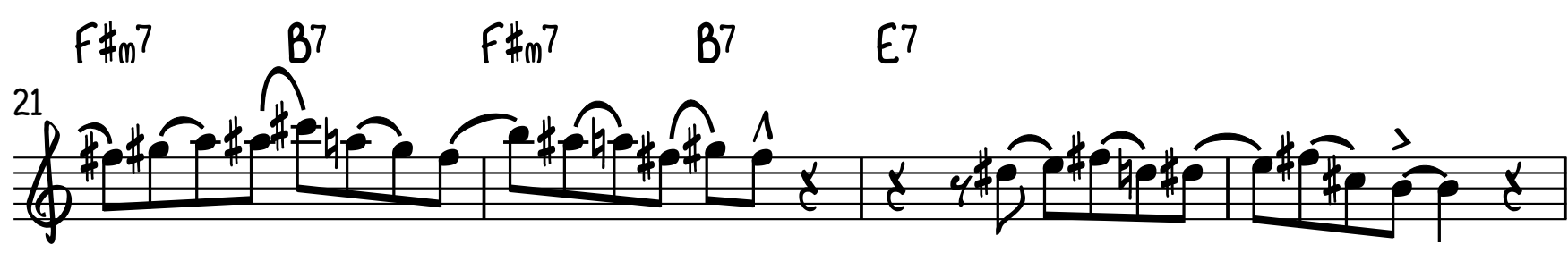

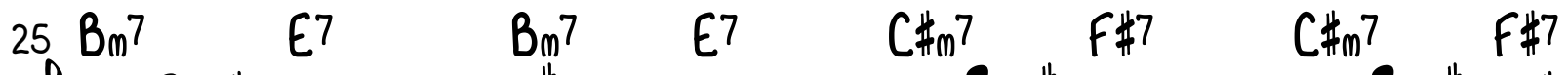

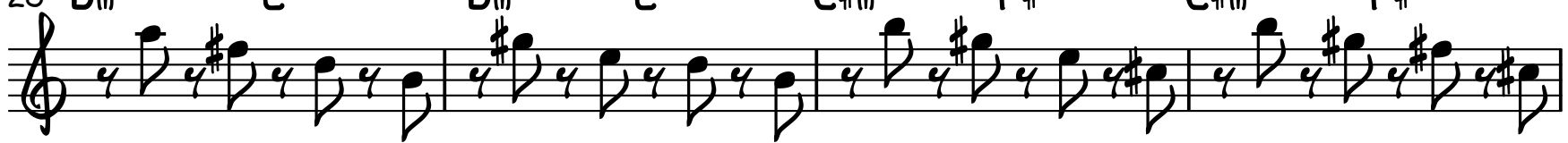

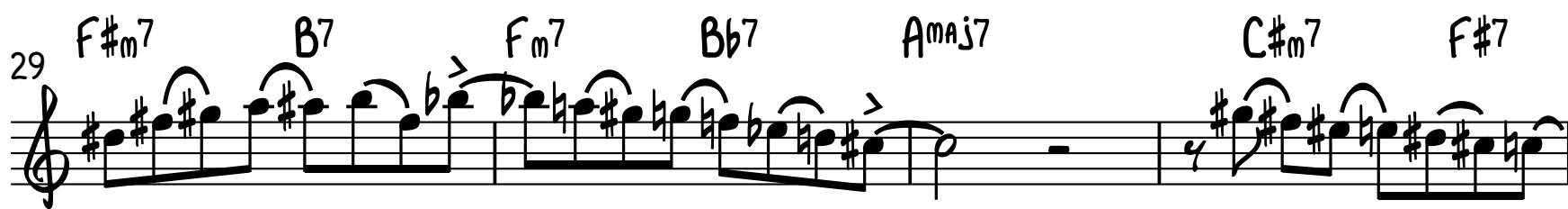


ALTO SAXOPHONE

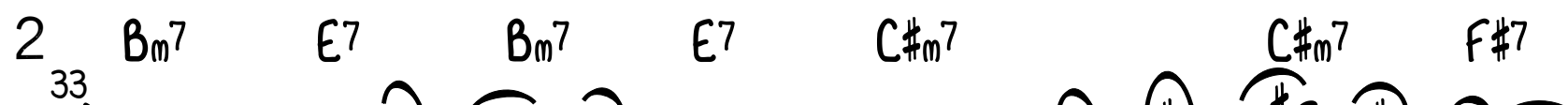
6)

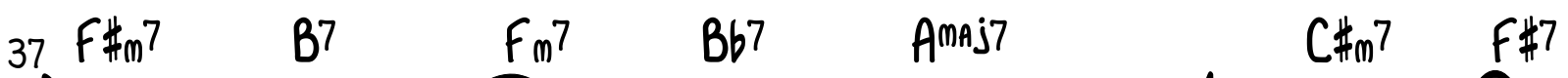
(4)

$\begin{array}{llllllll}B_{m} 7 & E 7 & B_{m} 7 & E 7 & C \#^{7} & F \# 7 & C \# m^{7} & F \# 7\end{array}$ 1)

$\mathrm{F}_{m} 7 \quad \mathrm{~B}^{7} \quad \mathrm{Fm}_{\mathrm{m}} \mathrm{B} \quad \mathrm{Bb7} \quad \mathrm{A}^{\text {maj7 }}$

(4)

$E_{m}{ }^{7} \quad A^{7} \quad E_{m}^{7} \quad A^{7} \quad D^{m a j 7}$

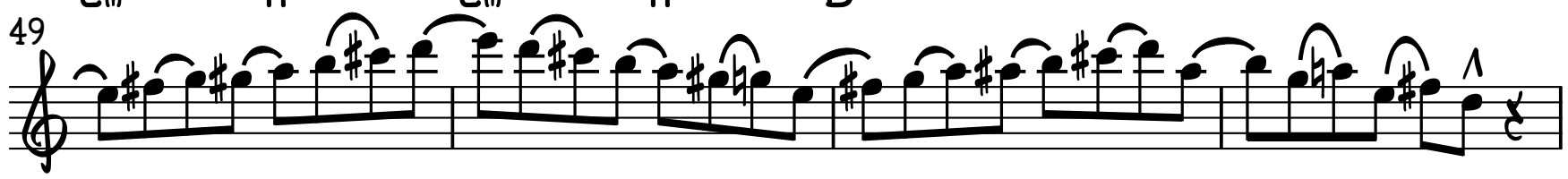

$53 F_{\# 7} 7 \quad B 7 \quad F \#_{m} 7 \quad B 7 \quad E^{7}$

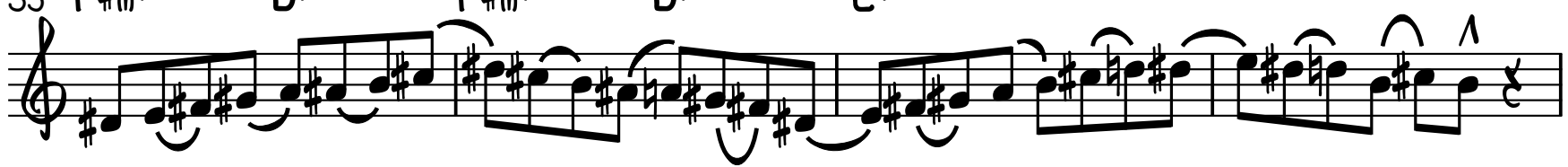

$B_{m} 7 \quad E 7 \quad B_{m} 7 \quad E 7 \quad C \# m^{7} \quad F \# 7 \quad C_{m} 7 \quad F \# 7$

(1)

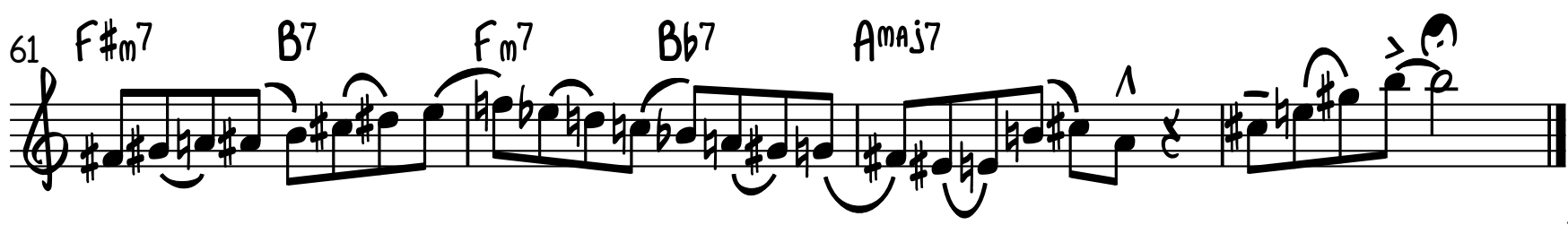


Exercises in Developing Bebop Scale Language

for Collegiate Jazz Students

\author{
Lars Swanson \\ West Virginia University
}

Trumpet in Bb Edition 


\section{SCALE EXERCISE 1 - MAJOR BEBOP SCALES}

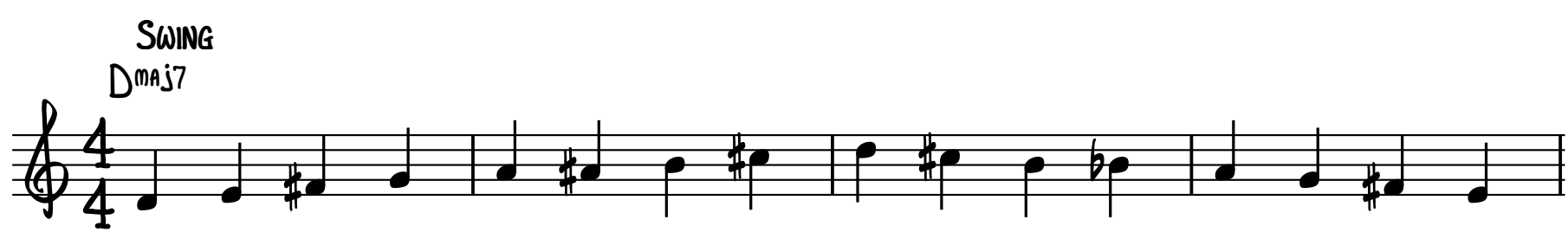

TRACK 1 - 100 BPM

TRACK 2 - 120 BPM

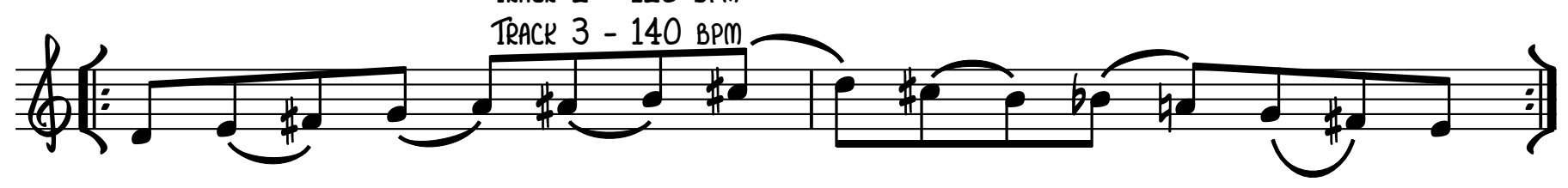
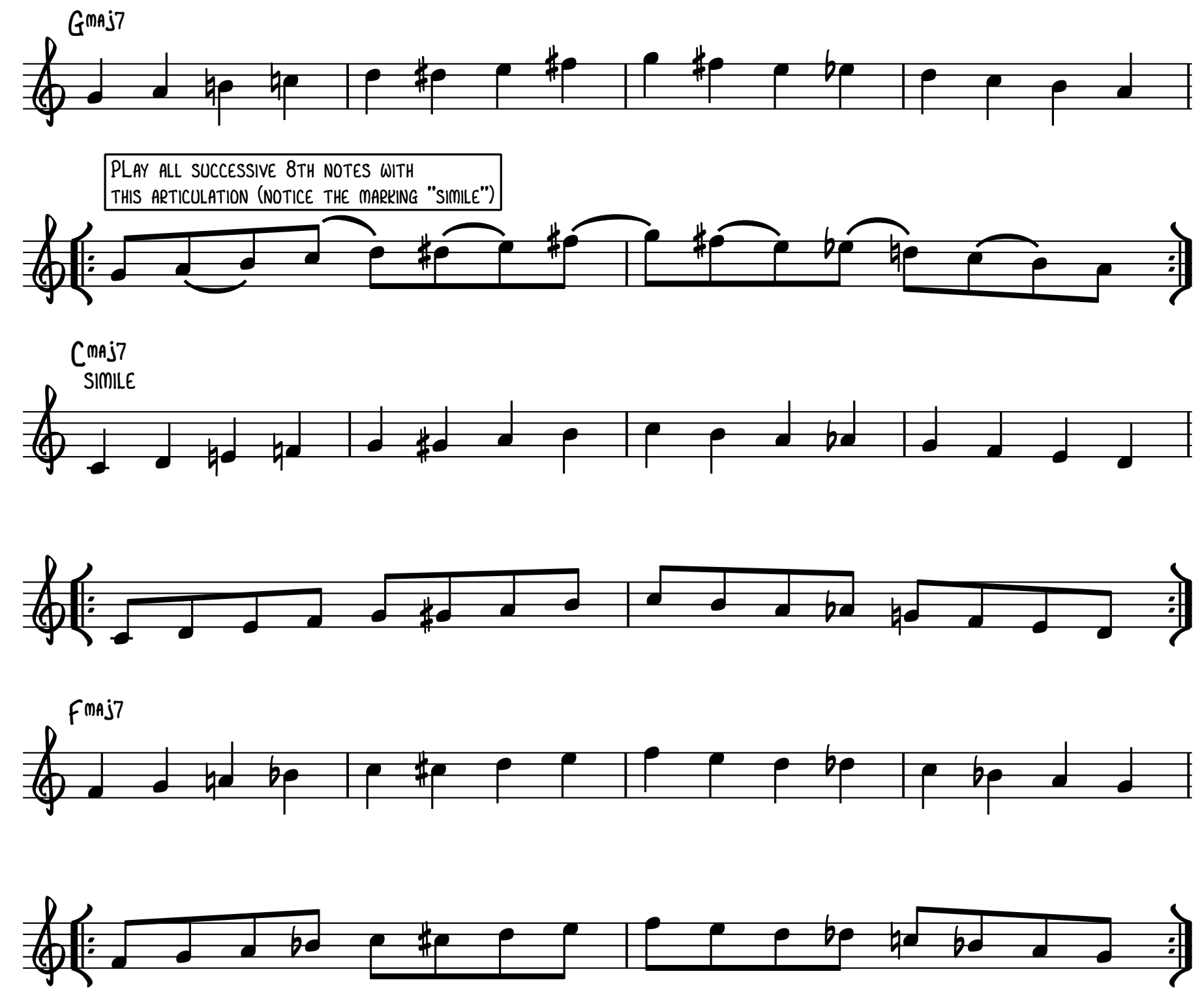


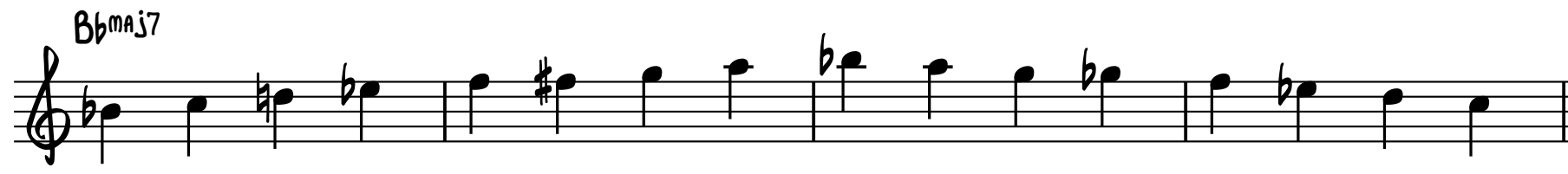

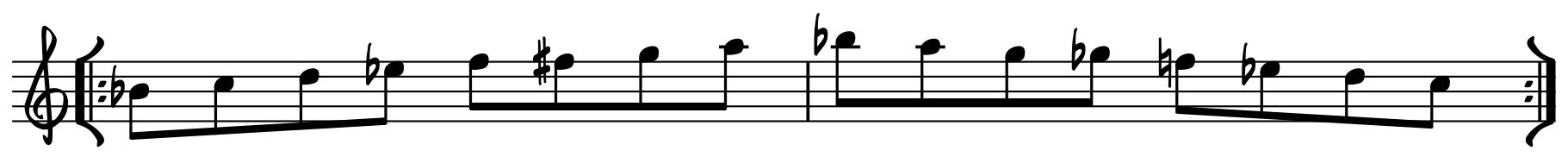

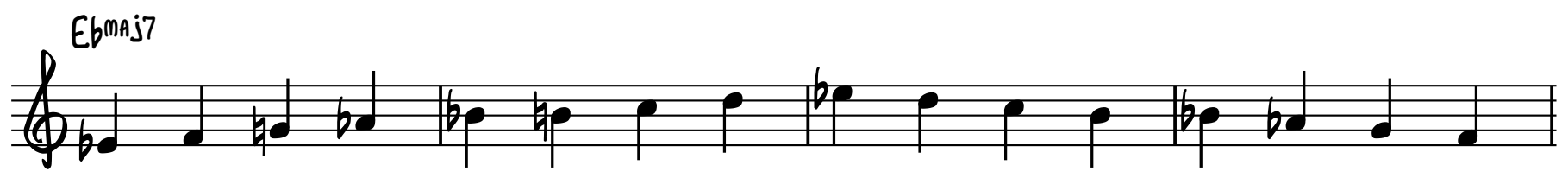

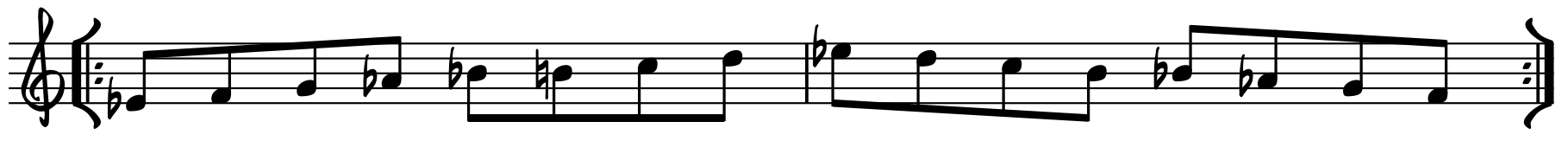

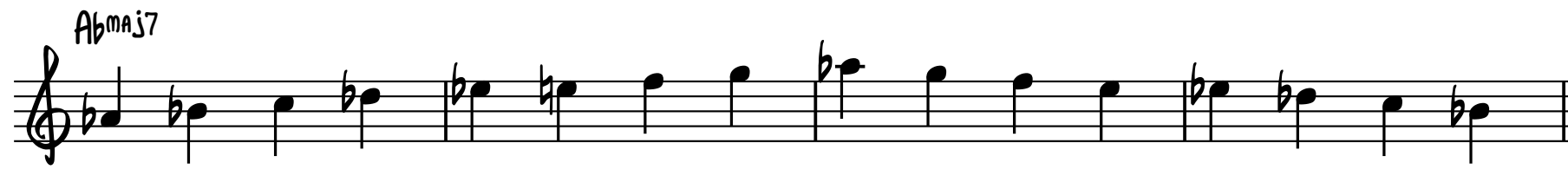

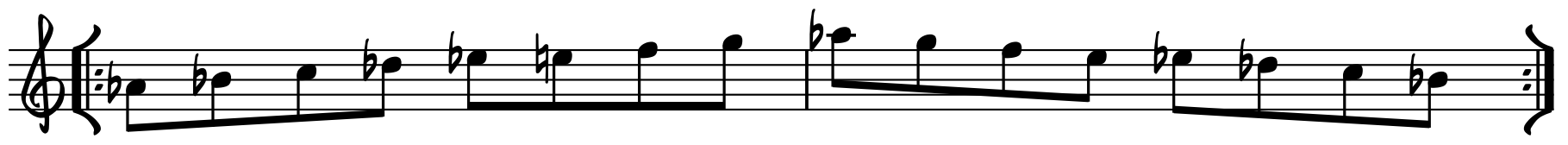

Dons

(4)

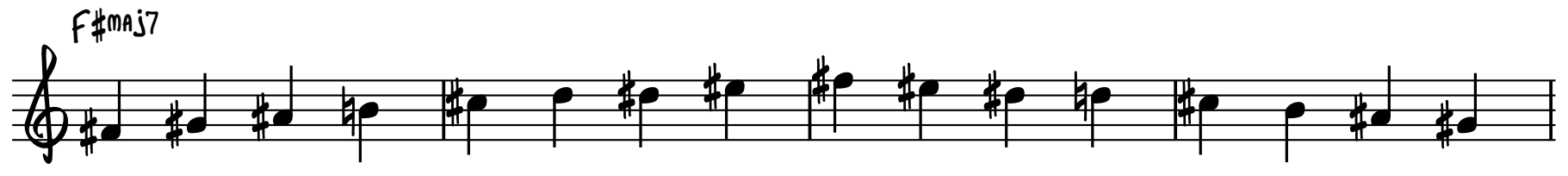

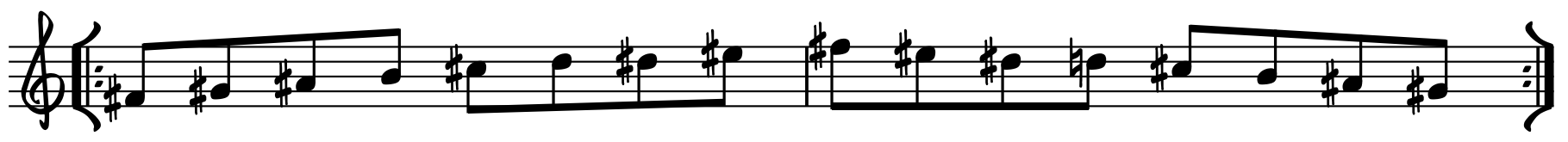



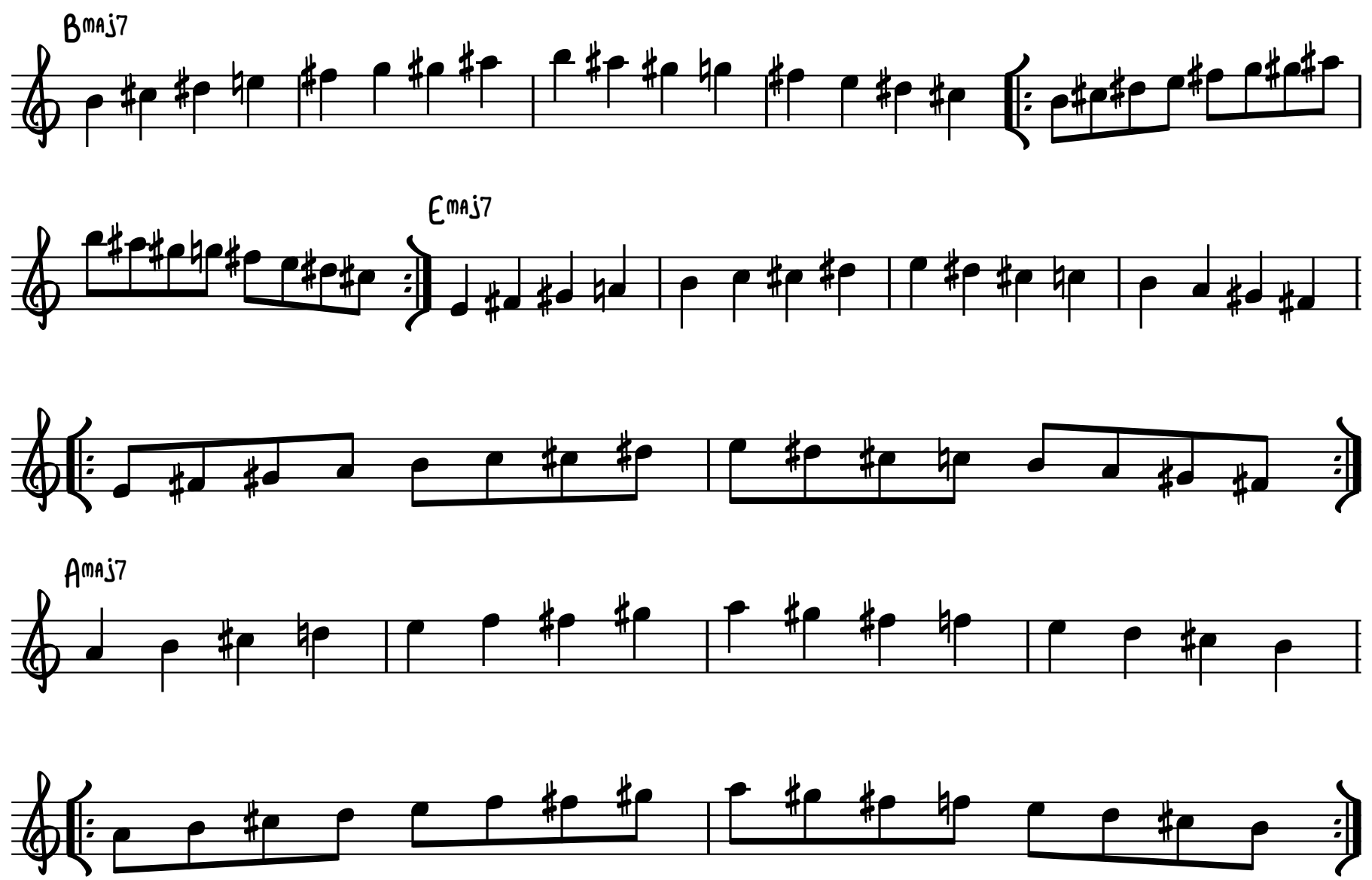
ONCE YOU HAVE MASTERED THIS AT THE FASTEST TEMPO, TRY TO
PRACTICE THESE SCALES STARTING ON THE 3RD, 5TH AND 6TH SCALE DEGREES.

CMAj7

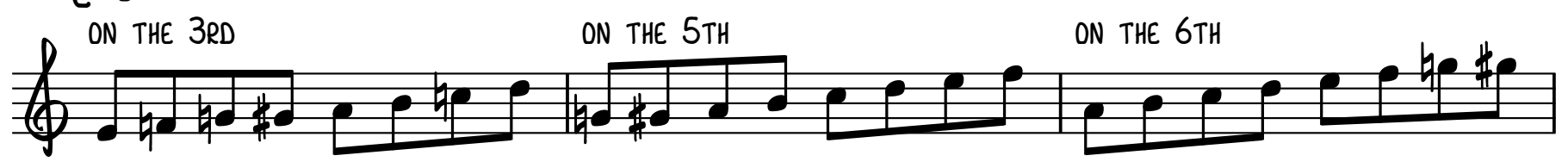




\section{SCALE EXERCISE 2 - DOMINANT BEBOP SCALES}

TRACK $10-100$ BPM TRACK 11 - 120 BPM

$\left.\right|_{1} ^{D 7}$

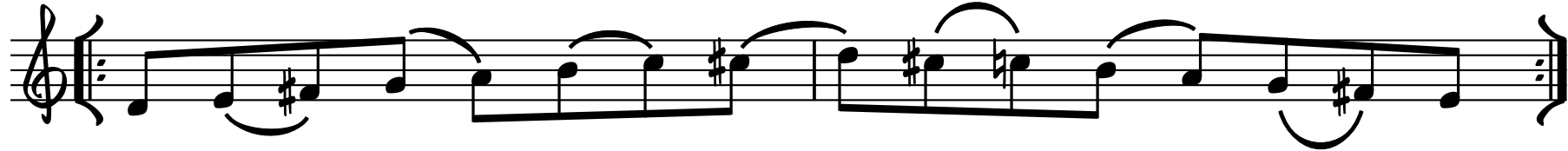

G7

(4)

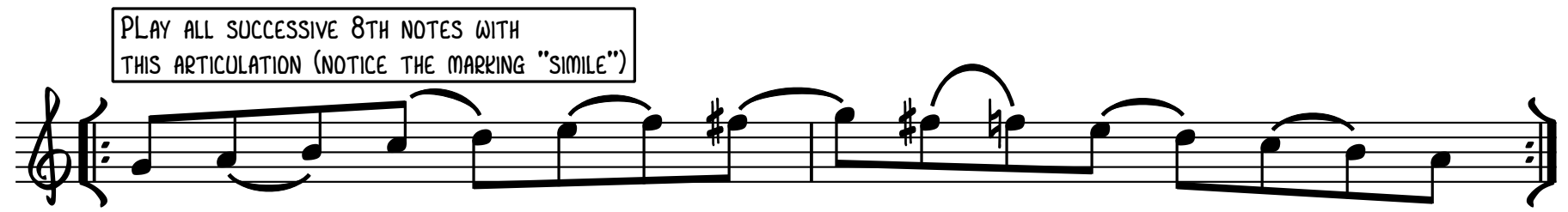

C7

$f^{\text {SIMILE }}$

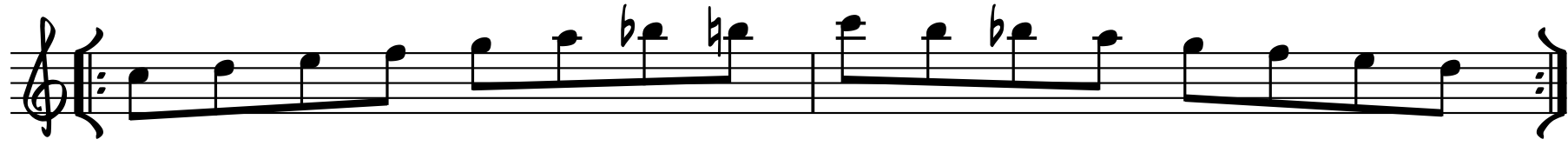

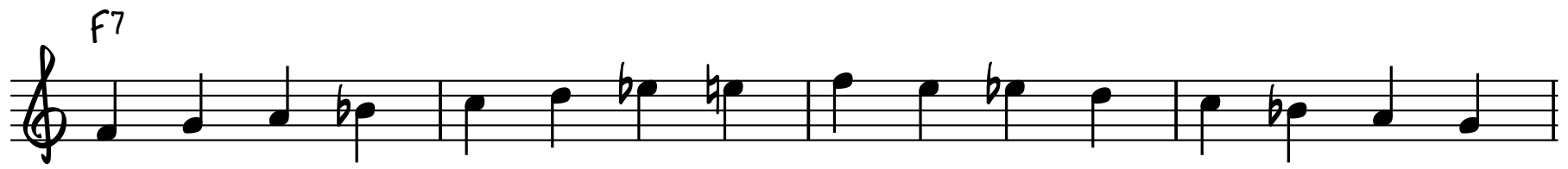

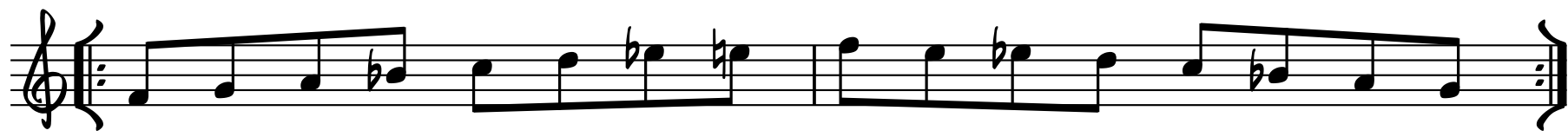

$f^{b p}$

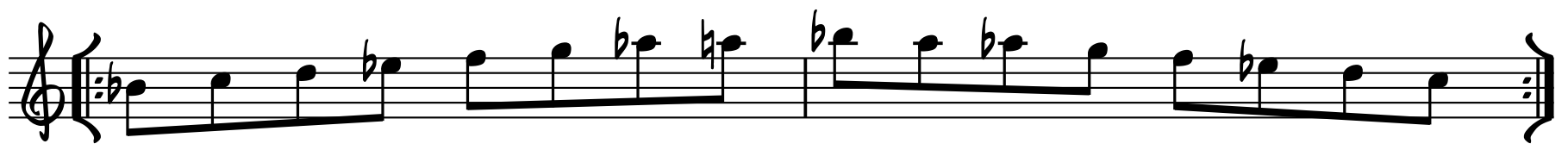


की

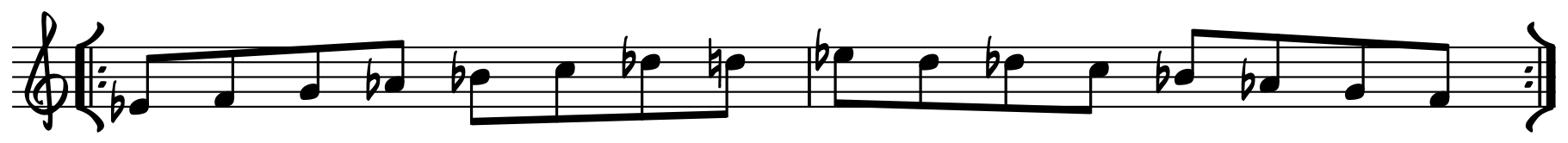

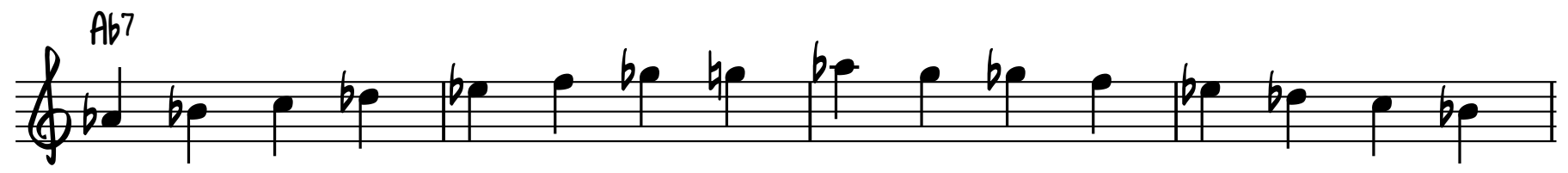

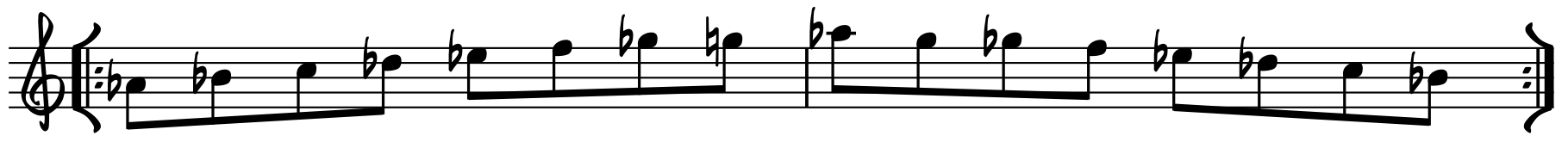

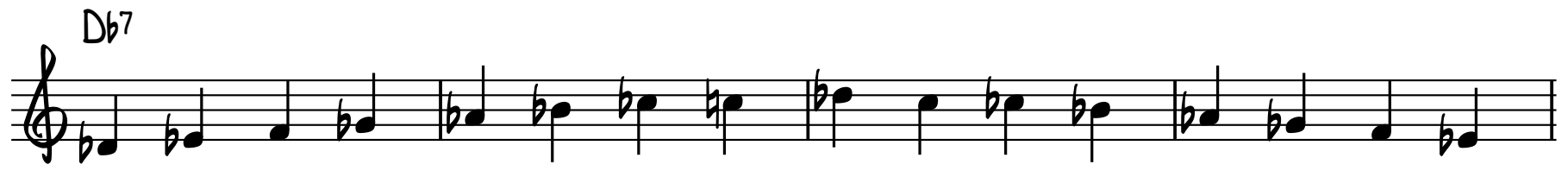

(4)

舟

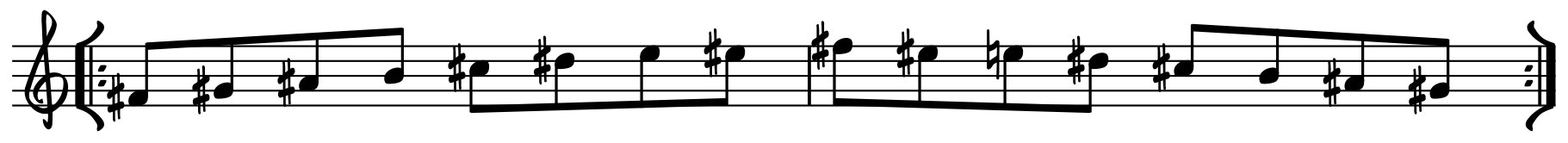

$e^{B 7}+\ldots$

(t) 

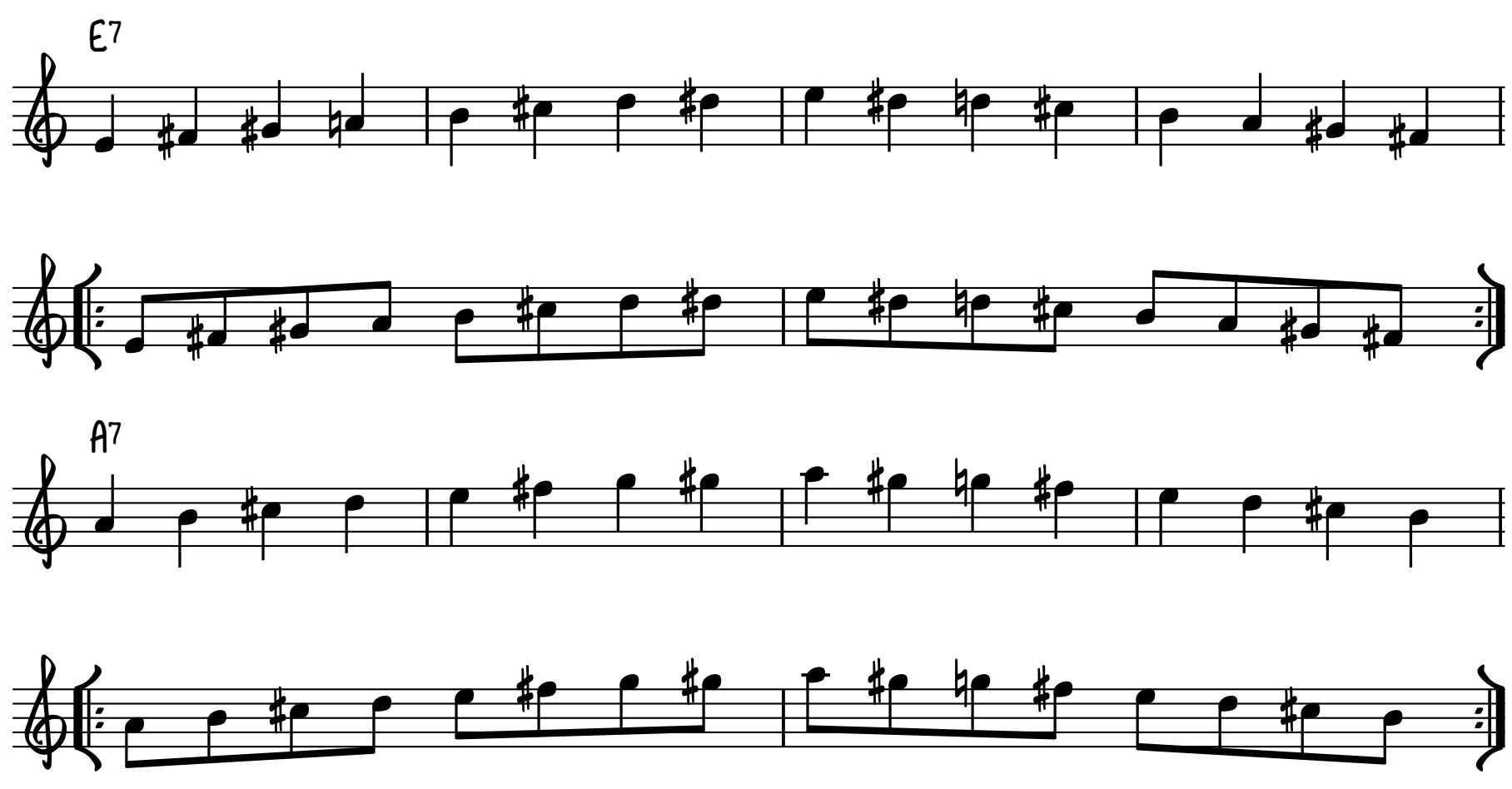

ONCE YOU HAVE MASTERED THIS AT THE FASTEST TEMPO, TRY TO PRACTICE THESE SCALES STARTING ON THE 3RD, TH AND FLAT TH SCALE DEGREES.

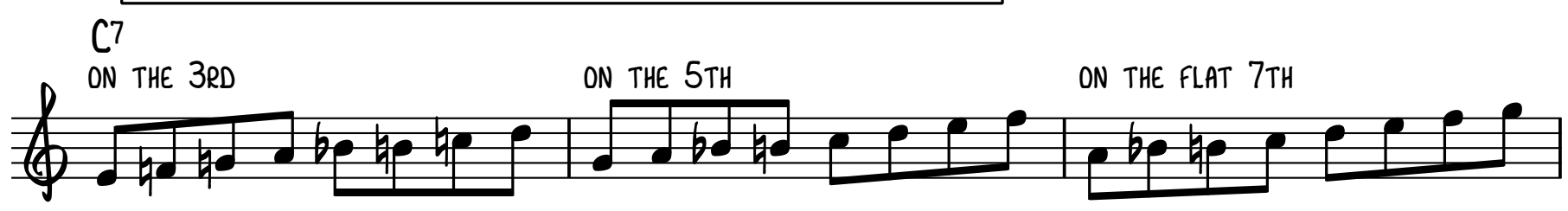




\section{SCALE EXERCISE 3 - MINOR BEBOP SCALES}

TRACK 19 - 112 BPM

$\begin{array}{lll}E_{m} 7 & A 7 & E_{m}^{7}\end{array}$

A7 TRACK $20-132 \mathrm{BPM}$

(4)

(2)

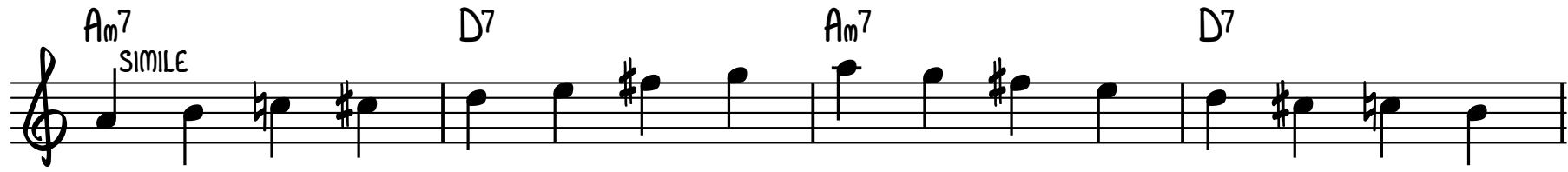

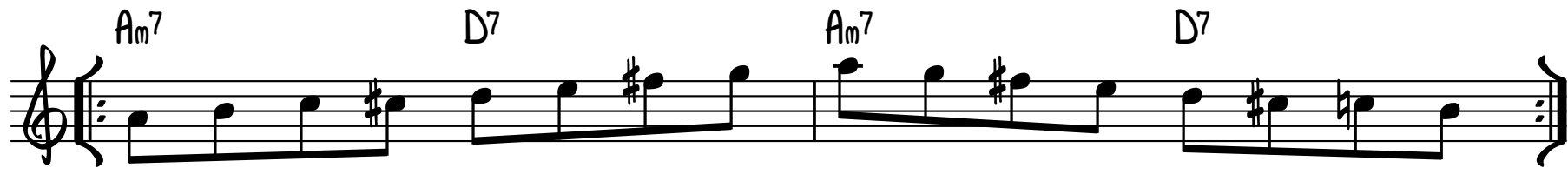

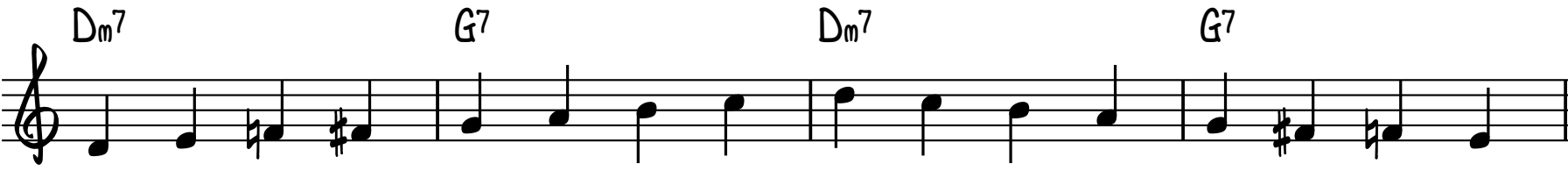

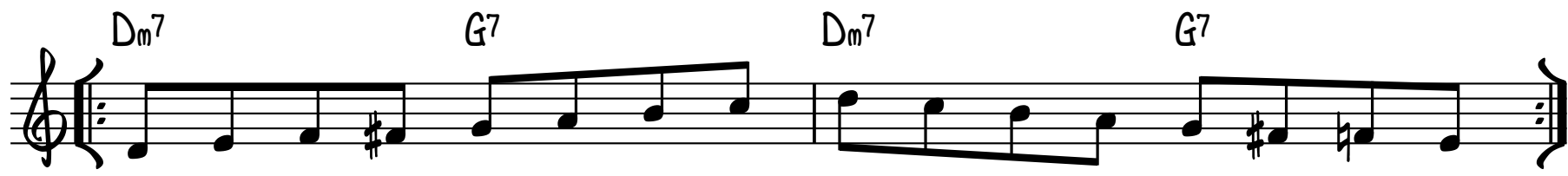

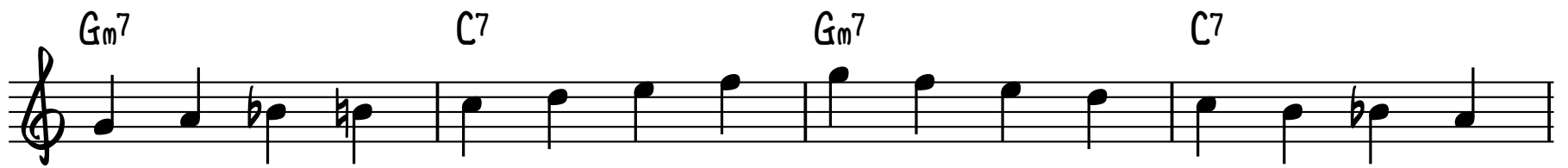

(4)

$f^{C_{m}{ }^{2}}$

$\underbrace{C_{m}{ }^{2}}$ 


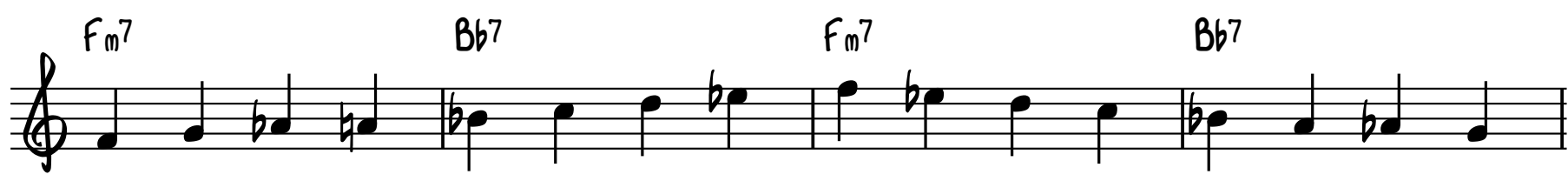

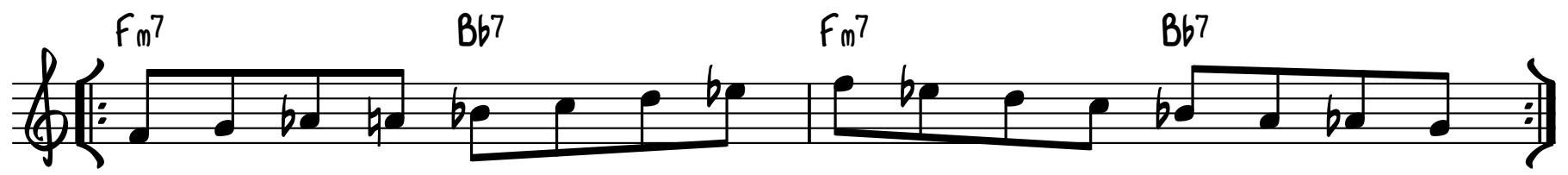

${ }^{B b r^{7}}$

${ }^{B b b^{7}}$

$b_{b b}^{E b_{n} 7}$

航(

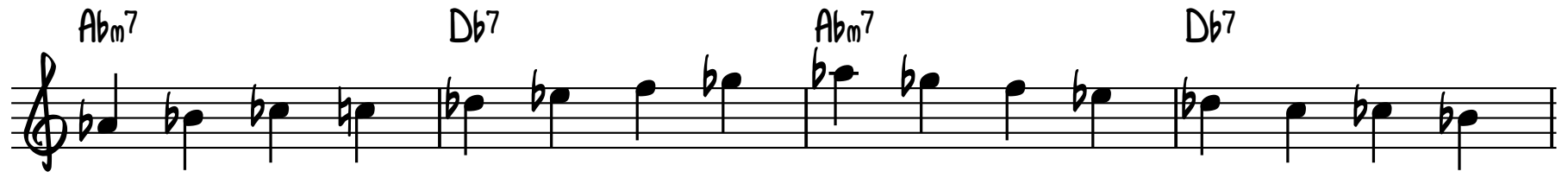

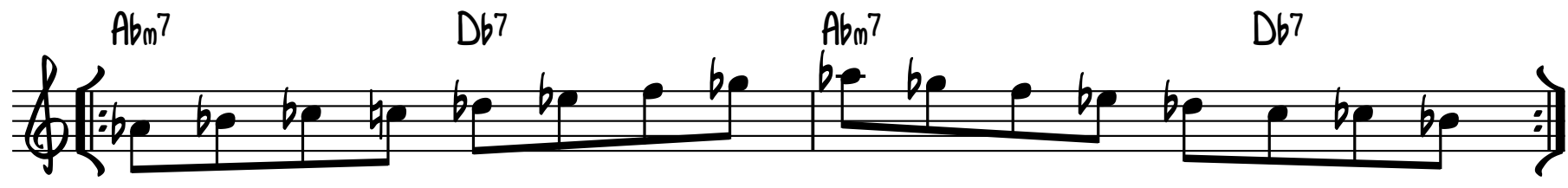

$\underbrace{C m_{n} 7}$

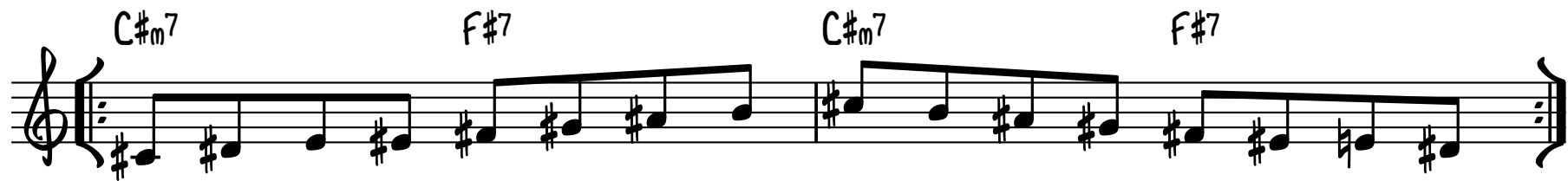



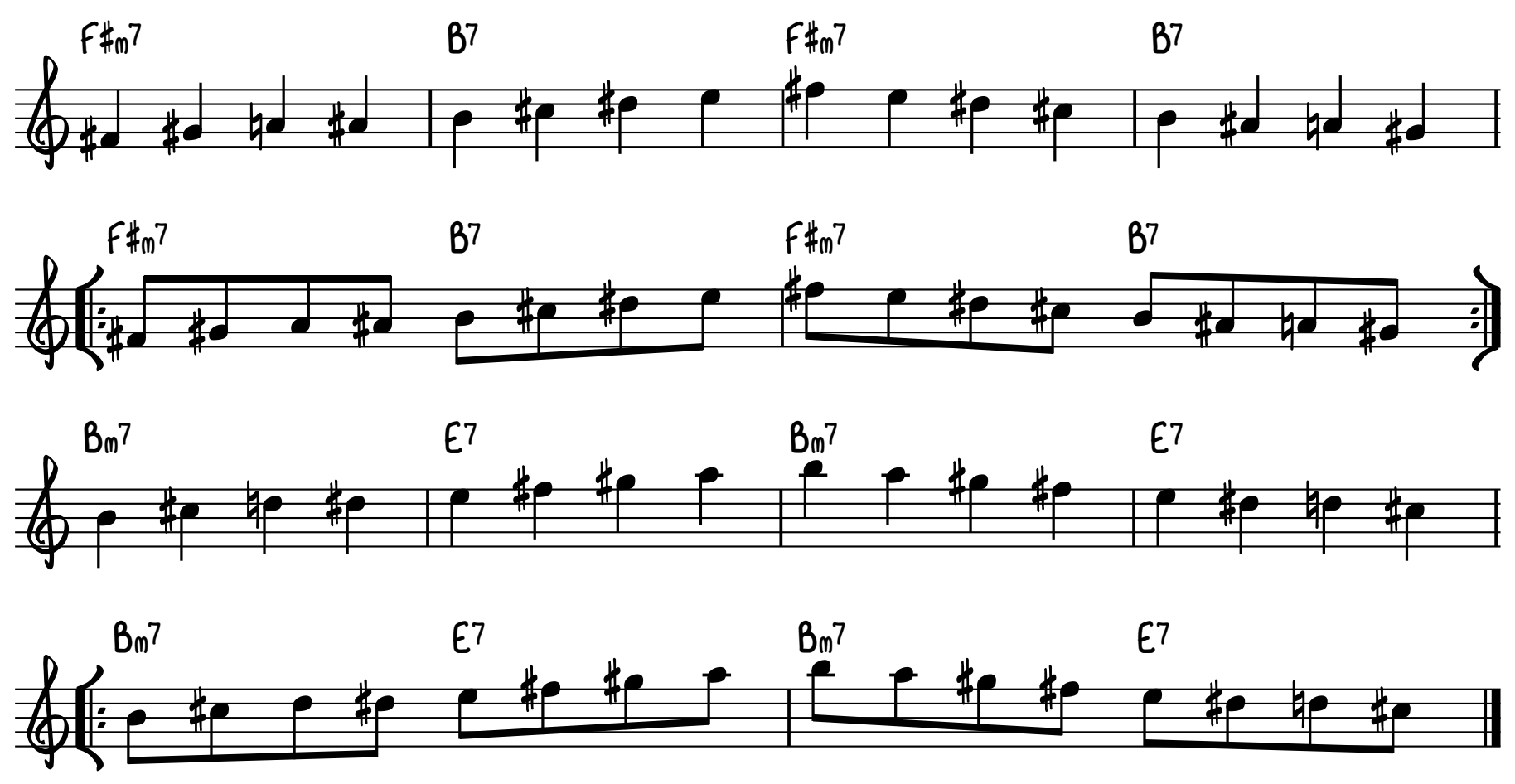

THIS IS THE 5TH MODE OF THE DOMINANT BEBOP SCALE. FOR FURTHER EXPLORATION, REFER TO THE EXPANSION IN SCALE EXERCISE 2. 
TRACK 22 - 100 BPM TRACK 23 - 120 BPM

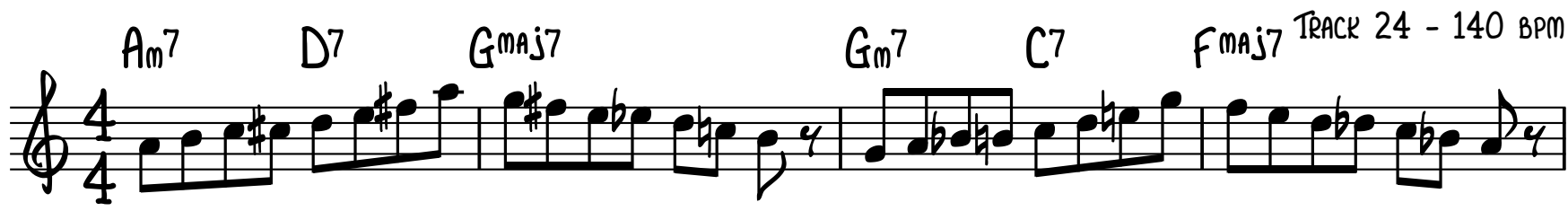
Bb7 Ebmaj7 Ebm Abri Ab7

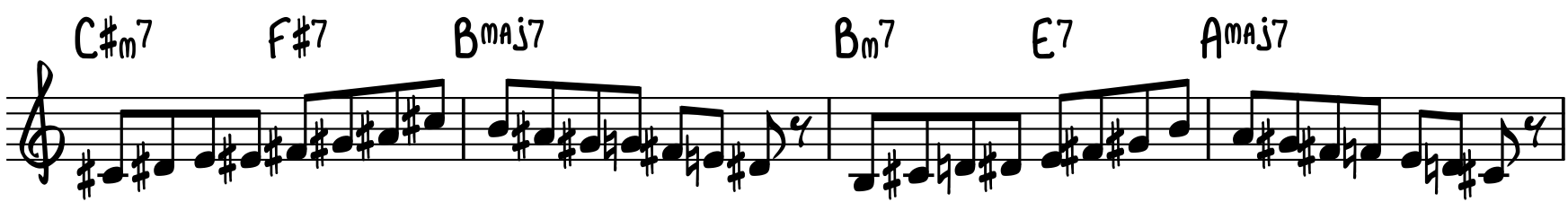
$\begin{array}{llllll}\mathrm{Cm}_{m} & \mathrm{~F} 7 & B b^{\text {maj7 }} & \mathrm{Bbm}^{7} & \mathrm{~Eb} 7 & \text { Abmaj7 }\end{array}$

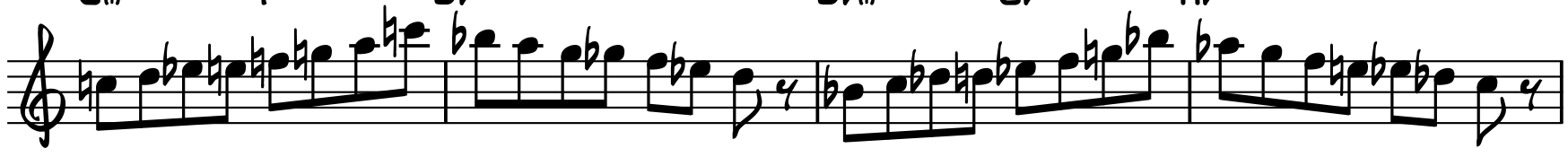

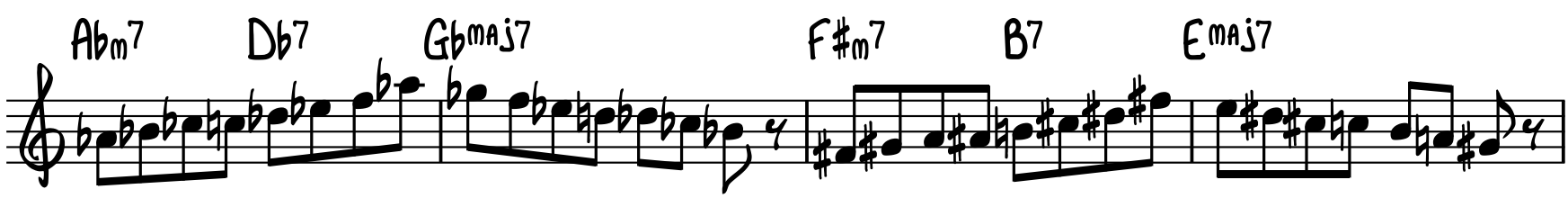
$\underbrace{E_{m}{ }^{A 7} D^{m a j 7}} D_{m 7}^{G 7}$ 

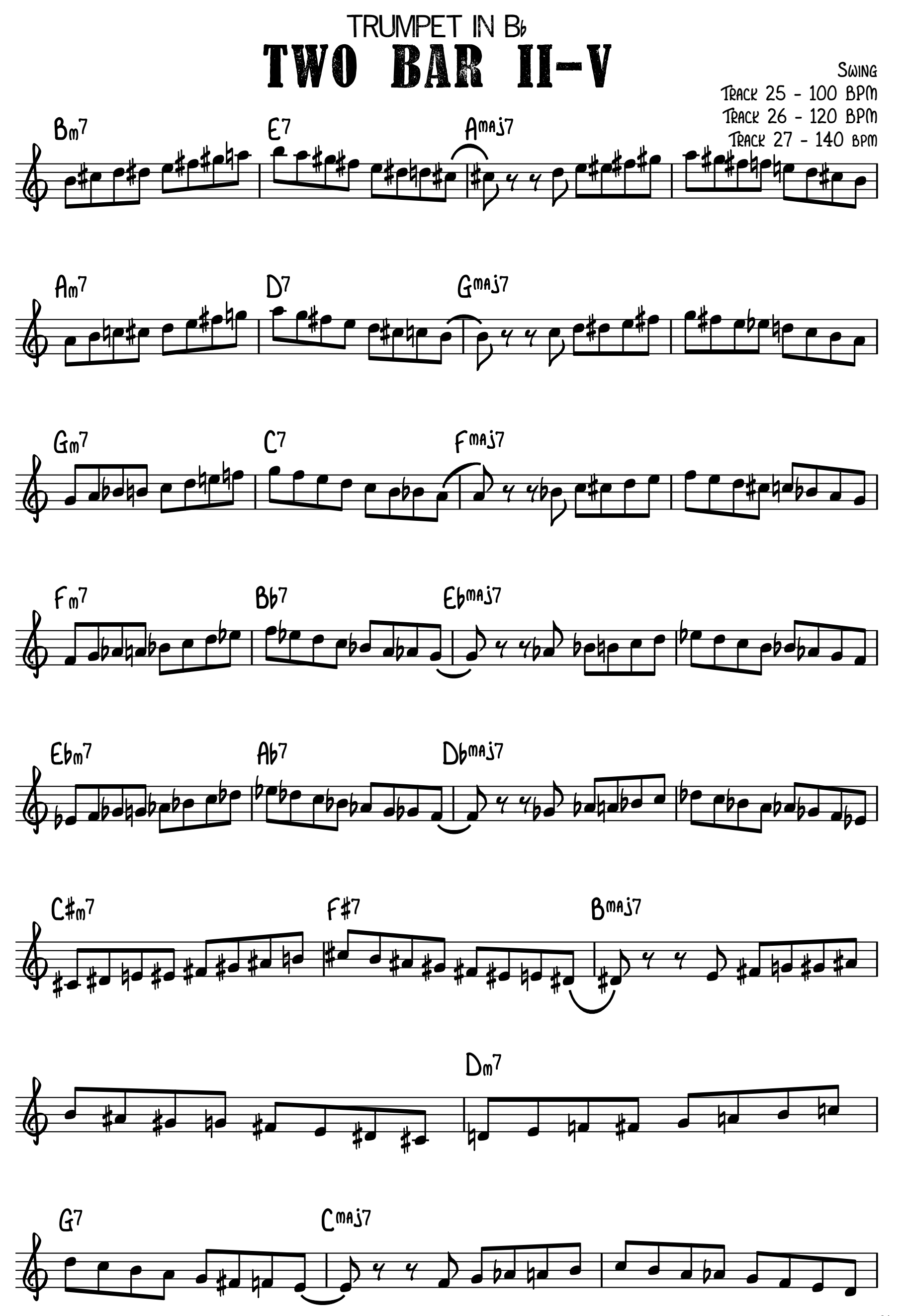
TRUMPET IN Bb

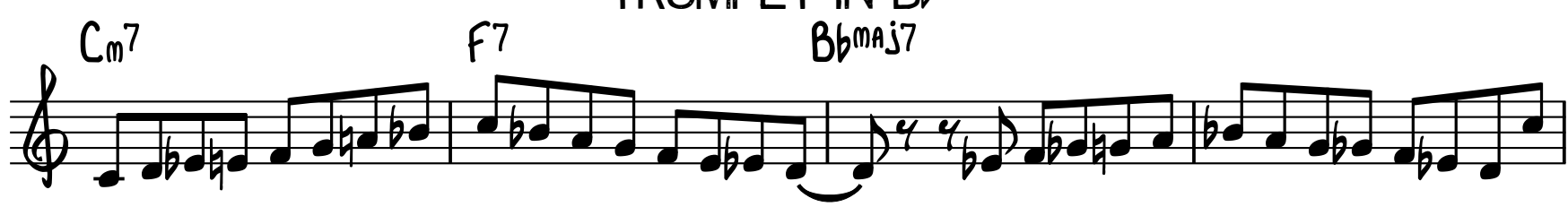

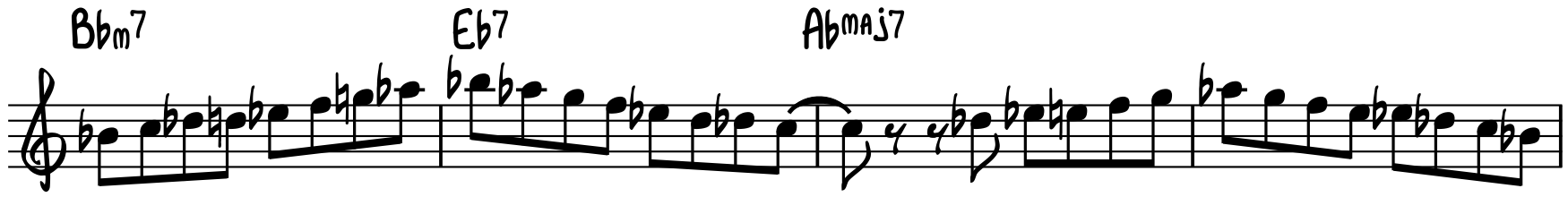

$f^{A b m 7} \begin{gathered}\text { Db7 } \\ \text { Gbopaj7 }\end{gathered}$

$\underbrace{F m^{7}}_{1}{ }^{B 7}$

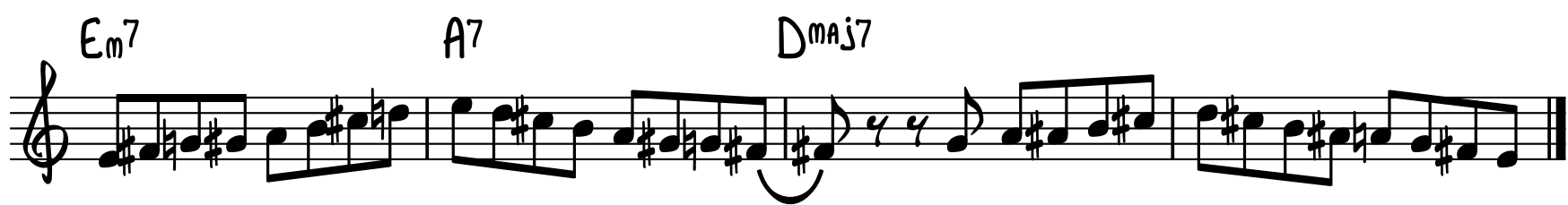




\section{TO IV MODULATION THROUGH II-V}

SWING

TRACK 28 - 92 BPM

TRACK 29 - 112 BPM

TRACK 30 - 132 BPM

$\underbrace{G \text { maj7 }} \mathrm{Dm}^{7} \mathrm{G}^{7}$

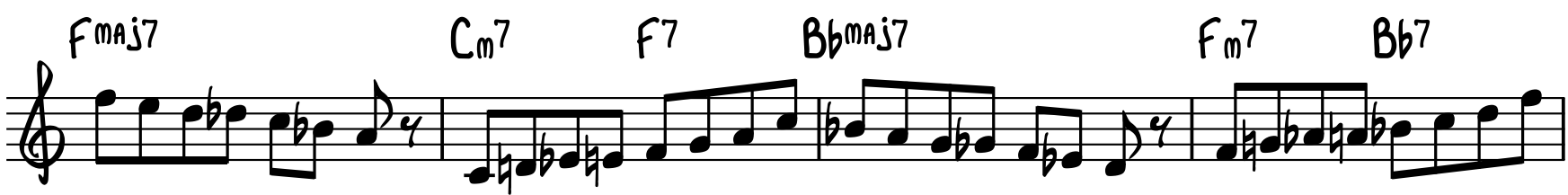

$\begin{array}{llllll}\text { Ebmaj7 } & B b m 7 & \text { Eb7 } & \text { Abmaj7 } & \text { Ebm } & A b 7\end{array}$

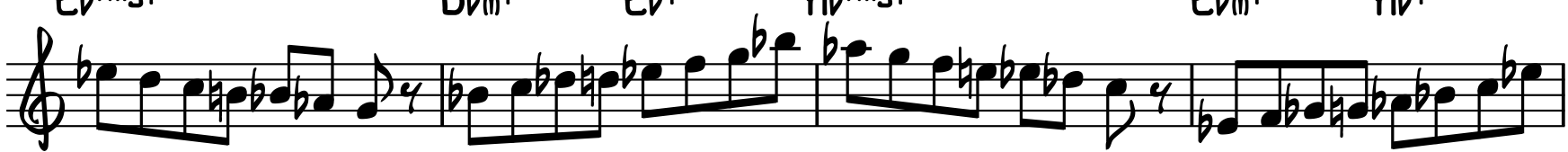

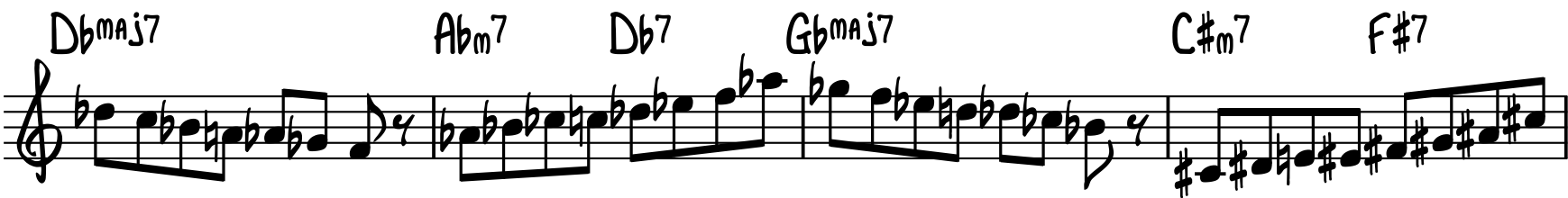

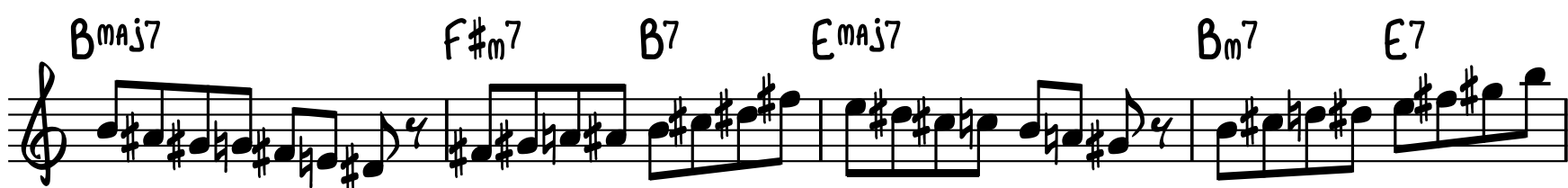

$\underbrace{A^{m a j 7}}$

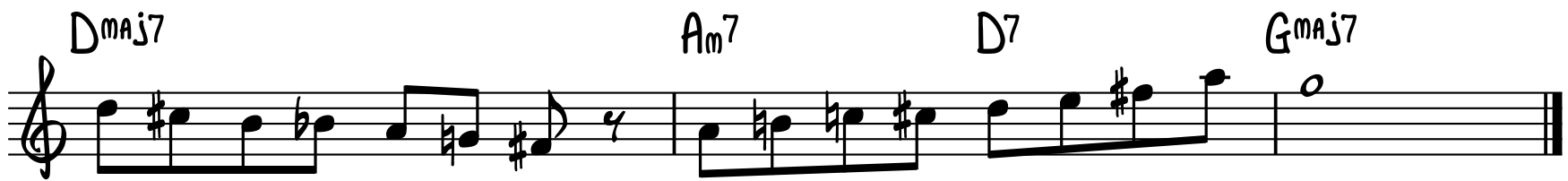


TRUMPET IN Bb

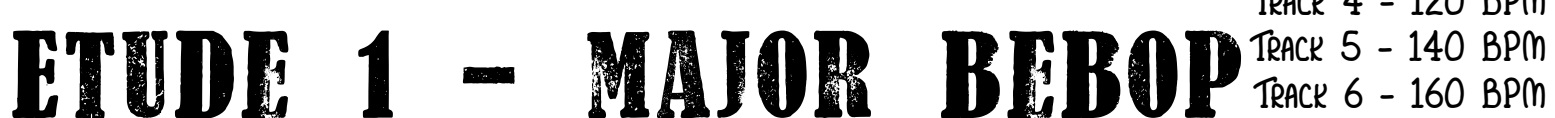
$\oiint_{4}^{4}$

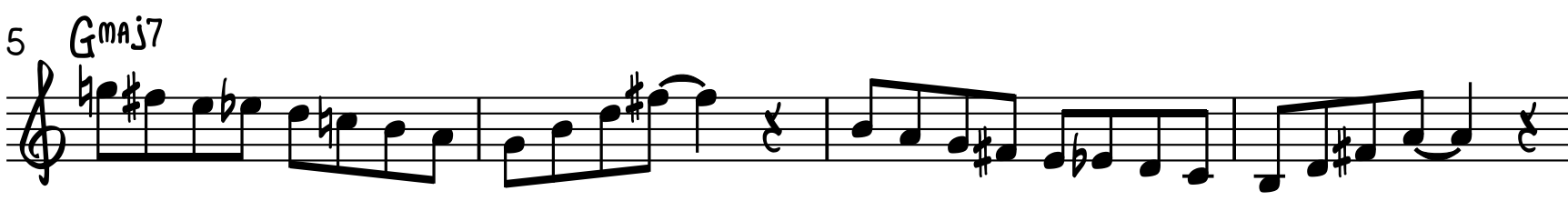

Fmaj7

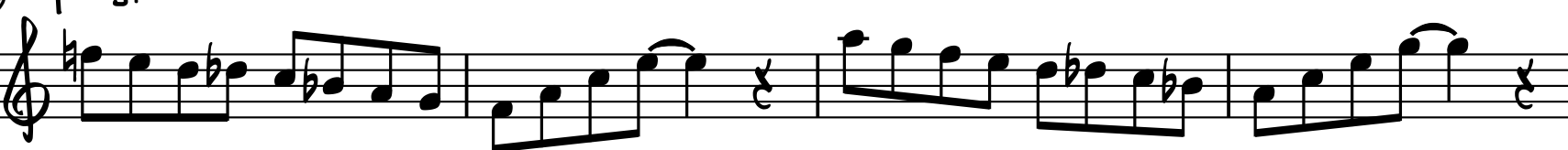

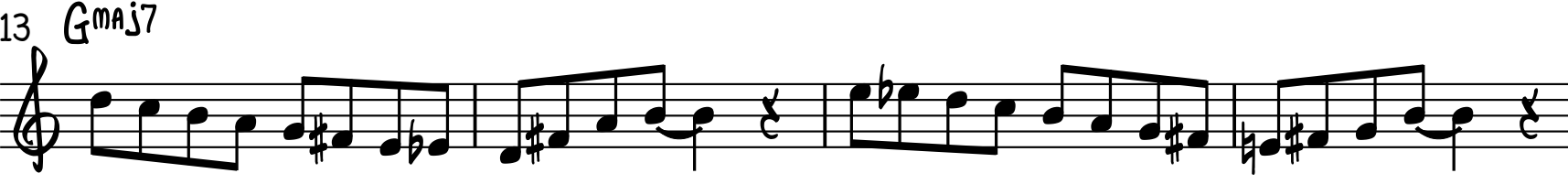
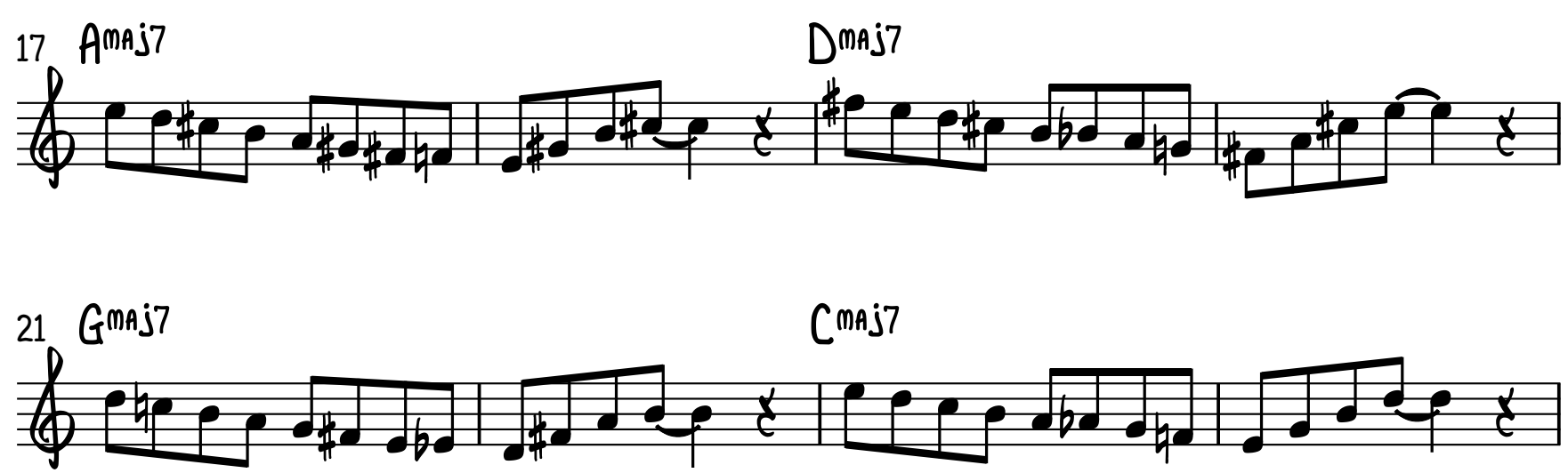

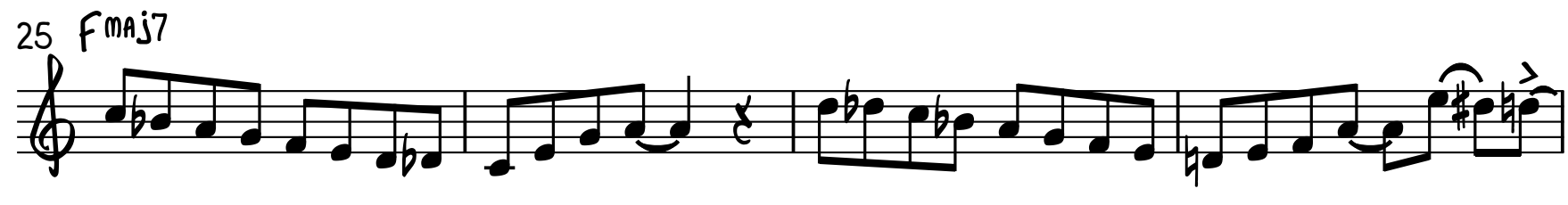

$29 \mathrm{G}^{\mathrm{maj} 7}$ 
2

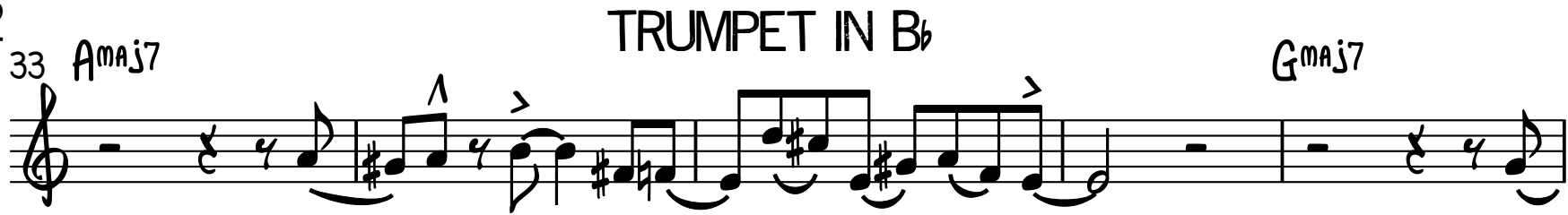
(4) 1 is (4) (4)

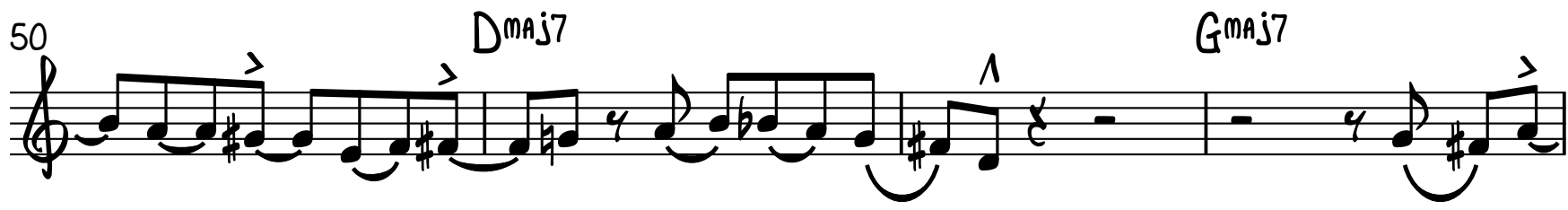

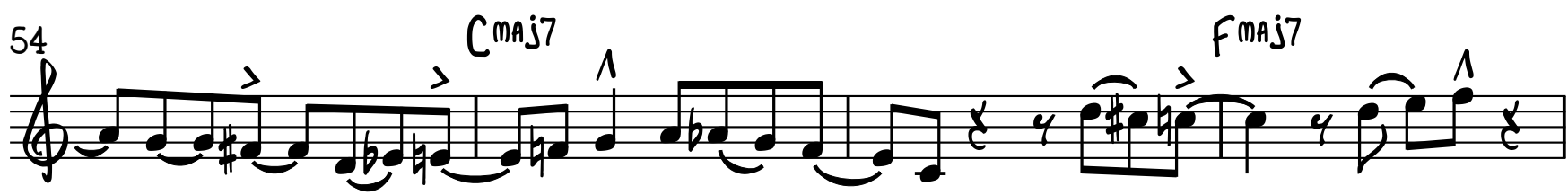
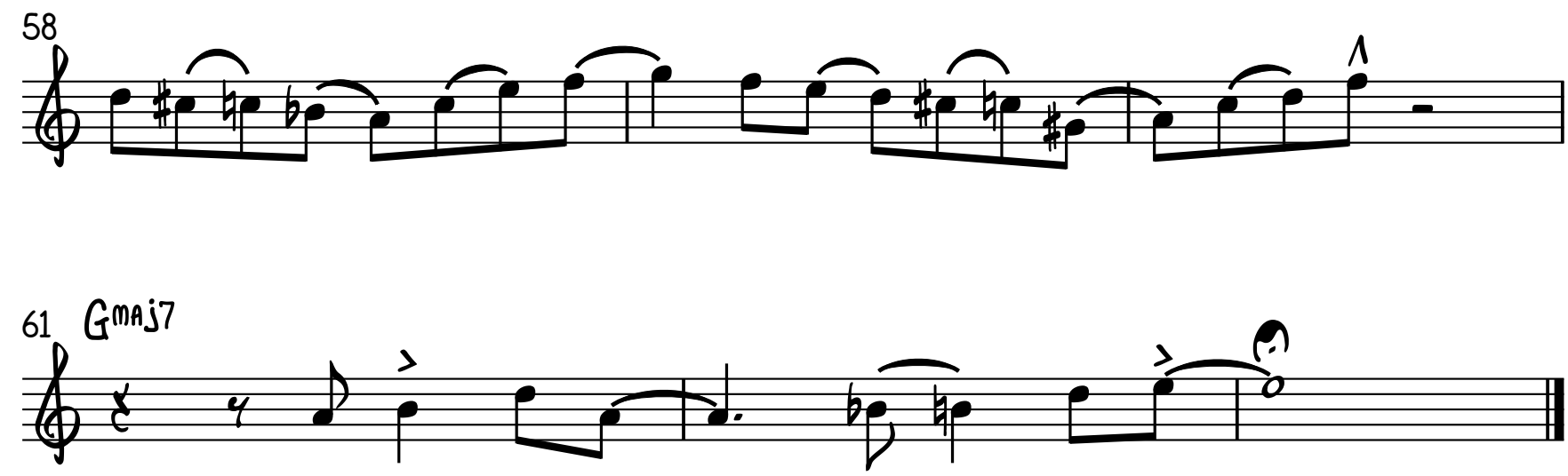
Tемuner $m$ в ETUDE 2 - MAJOR BEBOP
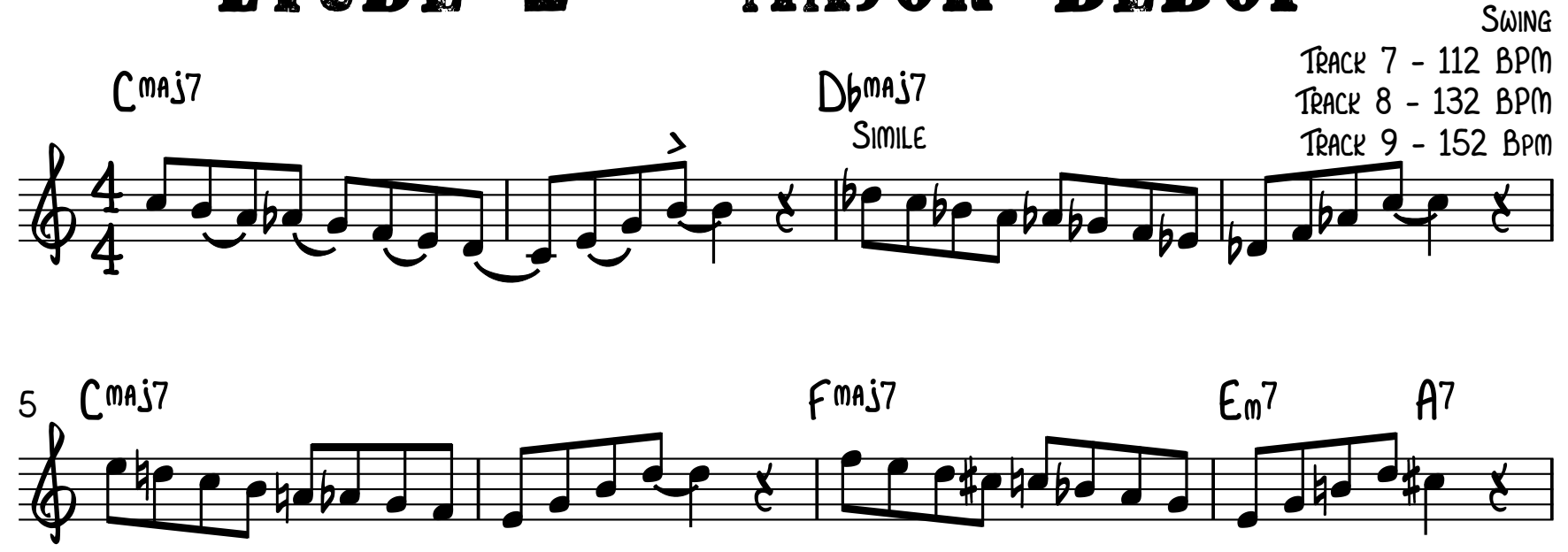

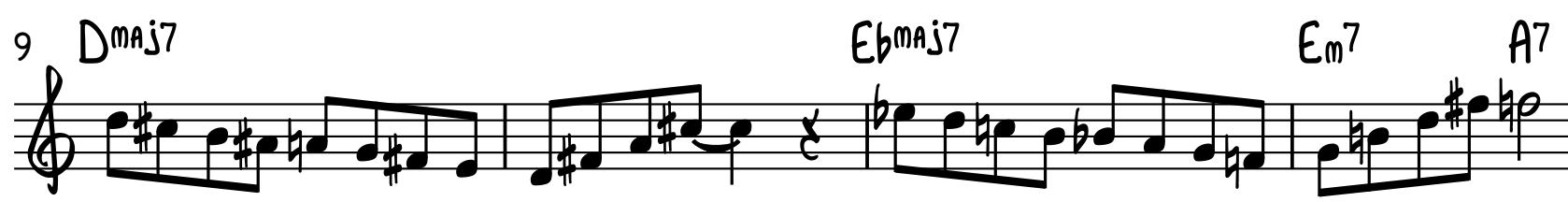

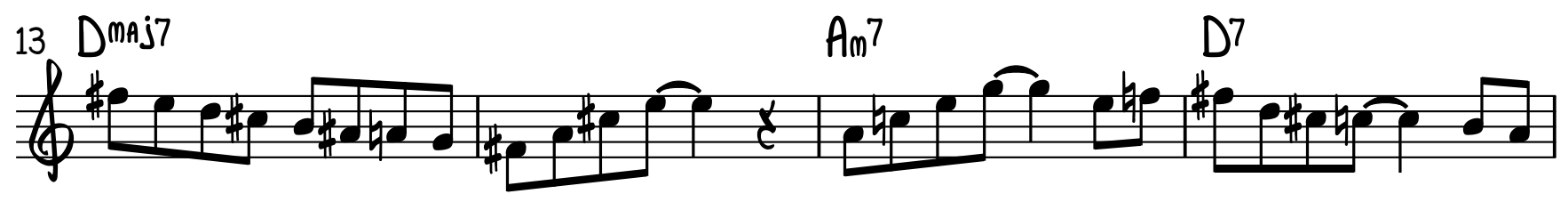

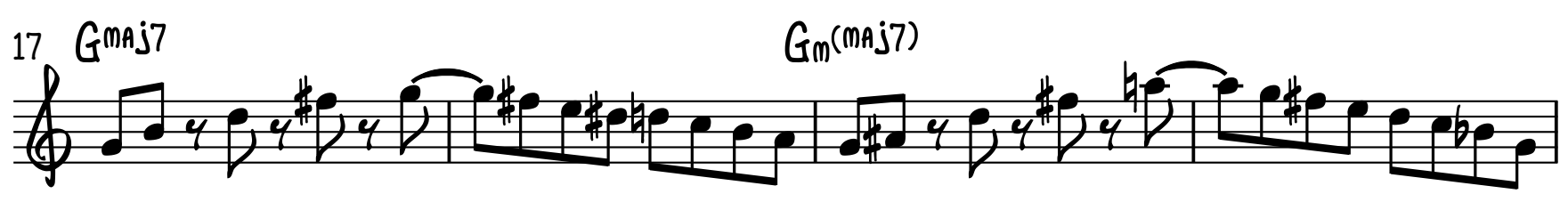
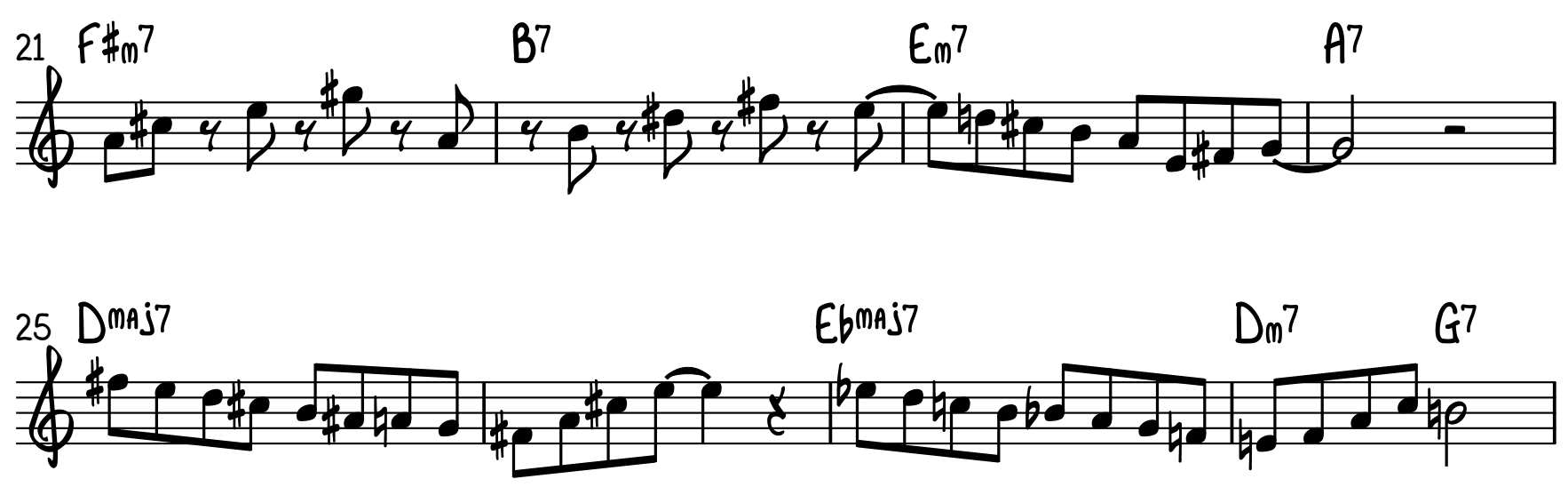

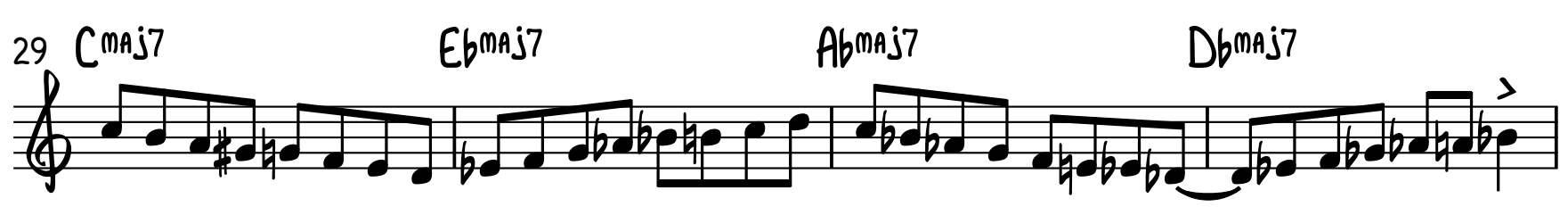



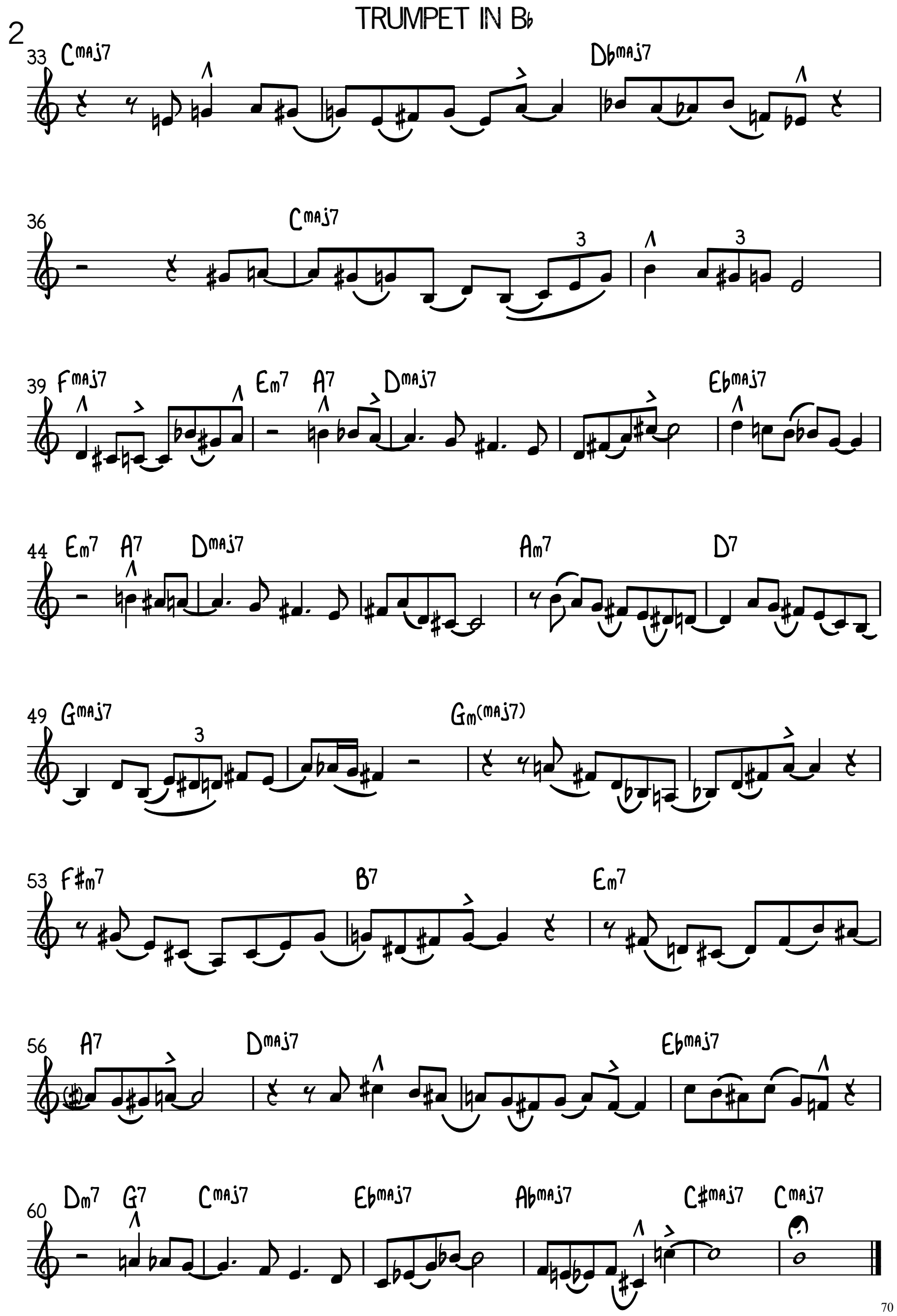
ETUDE 3 - DOMINANT BEBOP

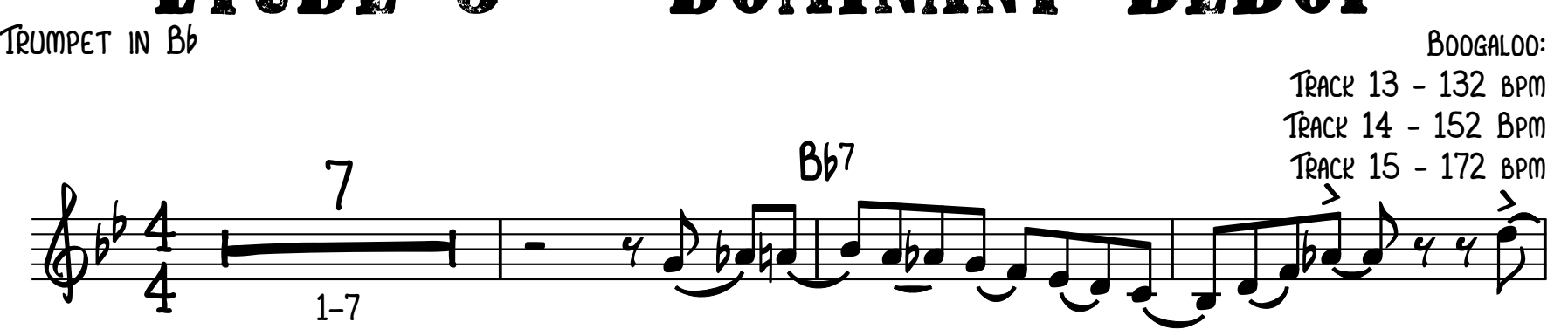

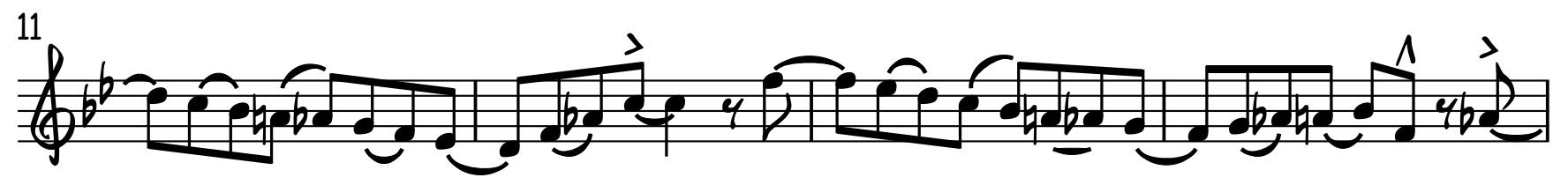

15 - At 7

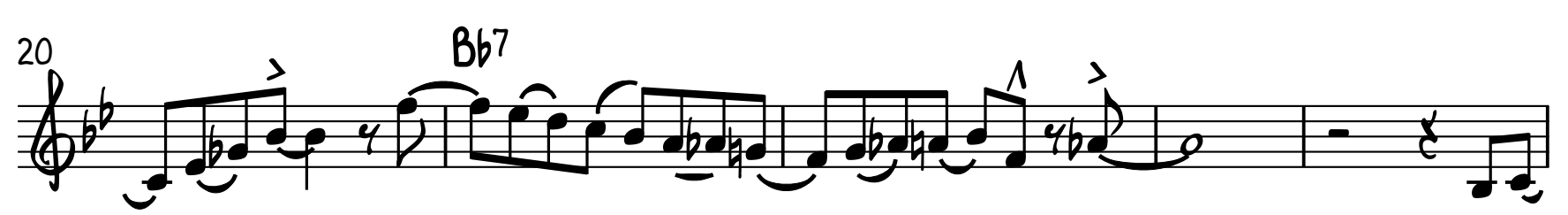

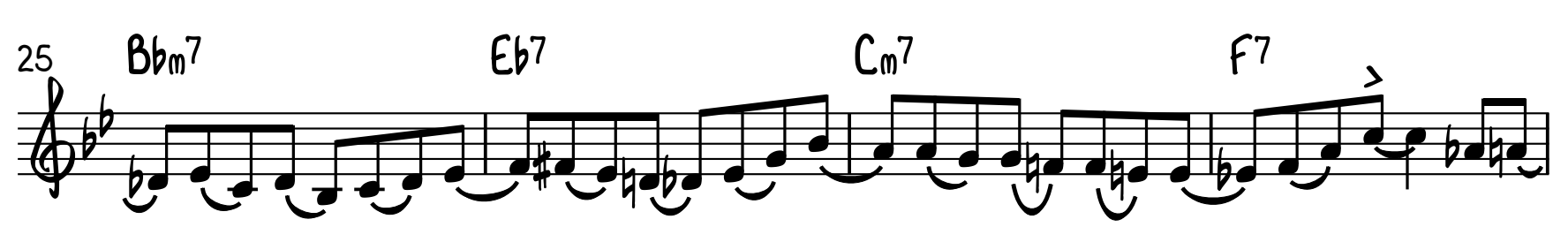

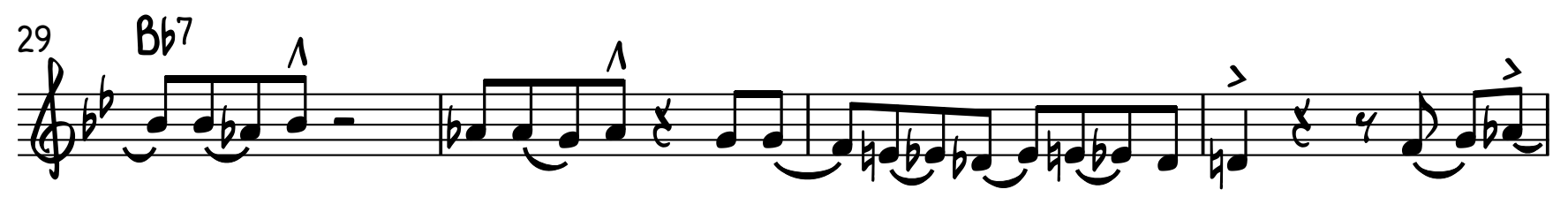


2 $\left.{ }^{233}\right)^{367}$ $\underbrace{37}$

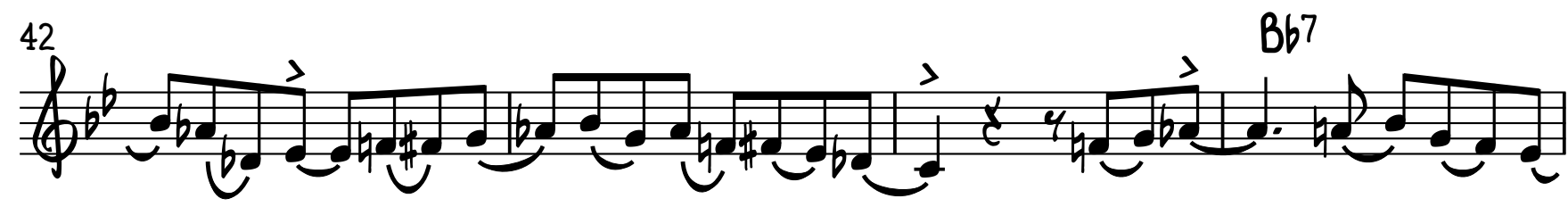

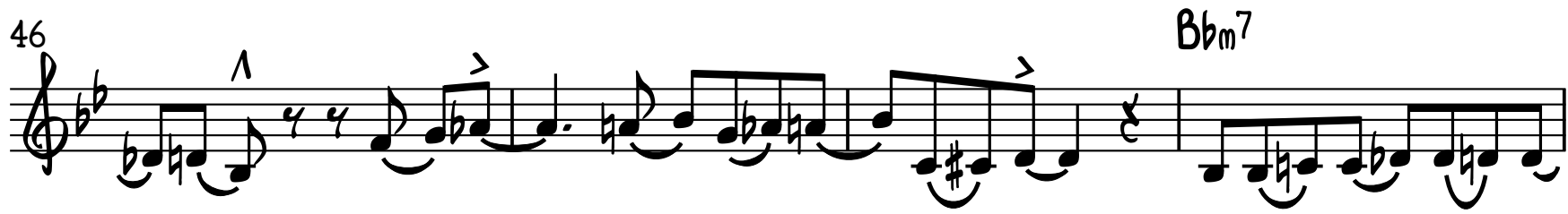

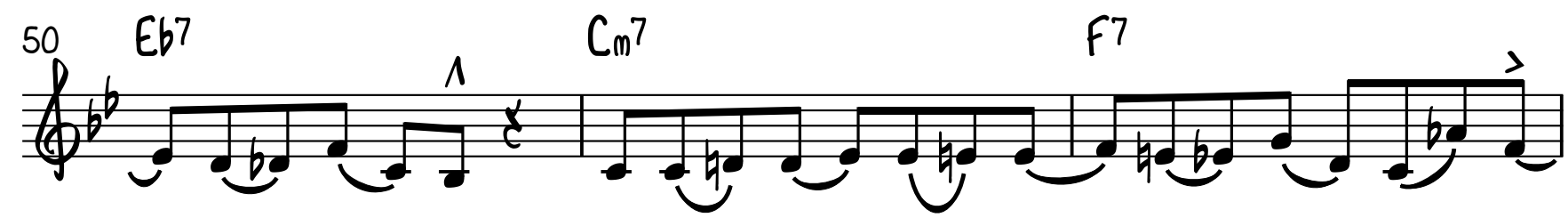

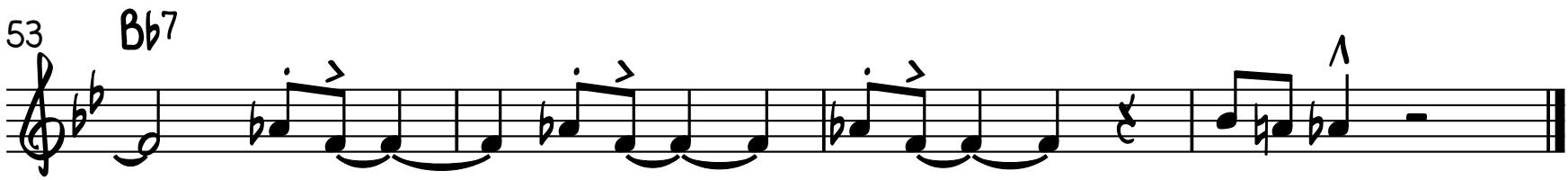




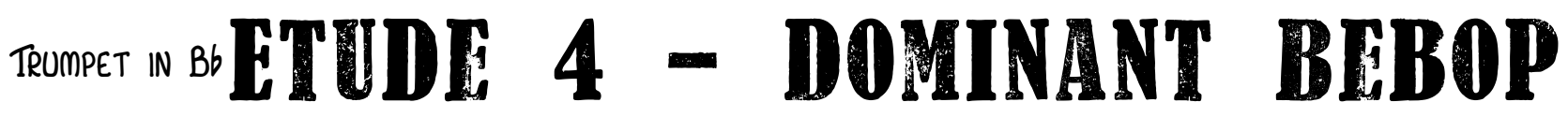

TRACK 16 - 112 BPM

D7

\&4 ${ }_{4}^{D^{7}}$

\&4 ${ }_{4}^{D^{7}}$

\&4 ${ }^{D^{7}}$

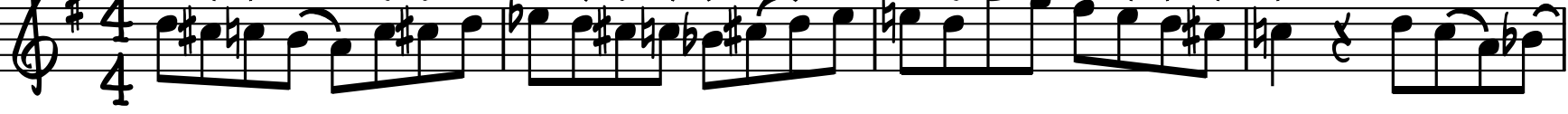

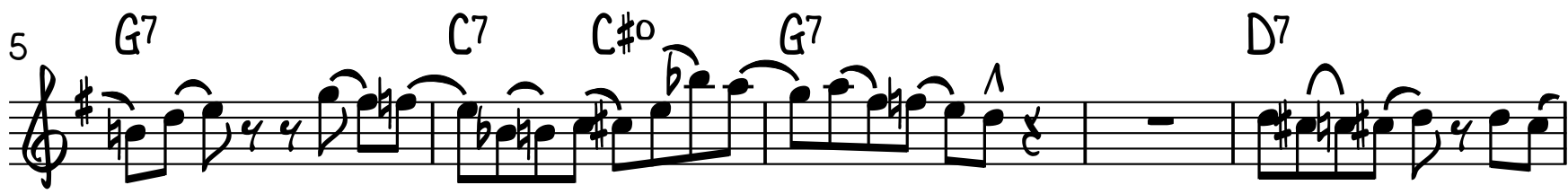

${ }^{10} \mathrm{~Eb}^{67}$

${ }^{14}{ }^{C 7}{ }^{C \# 0}$

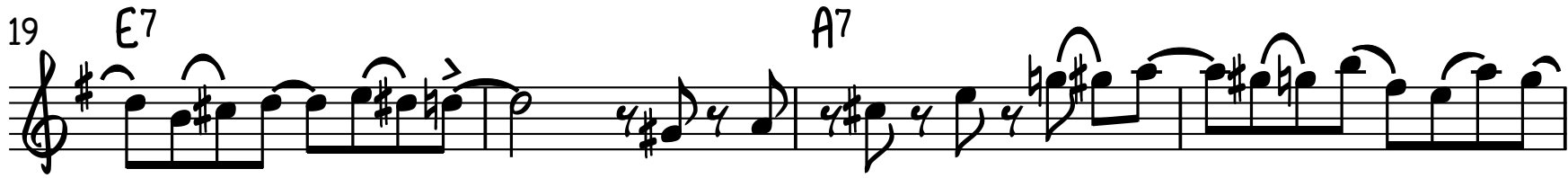

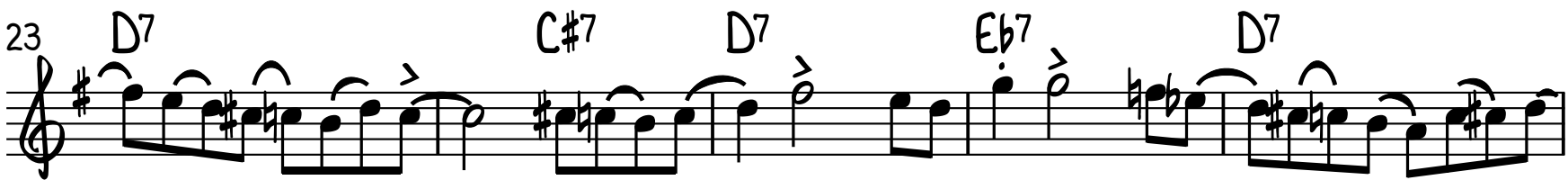

(4) 
2

TRUMPET IN Bb

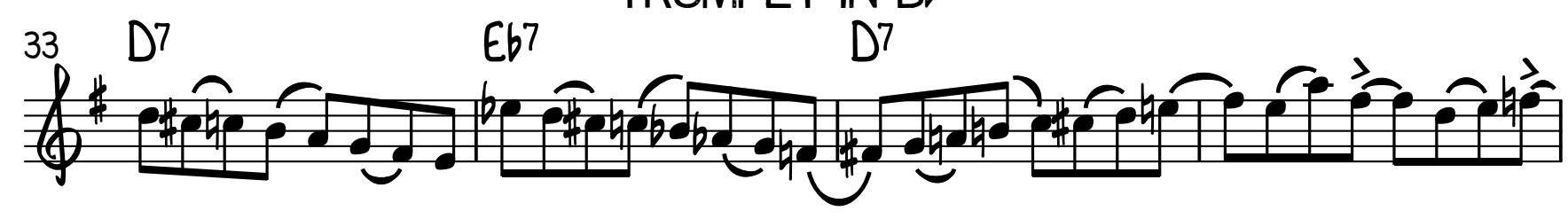

${ }^{37} G^{7}$

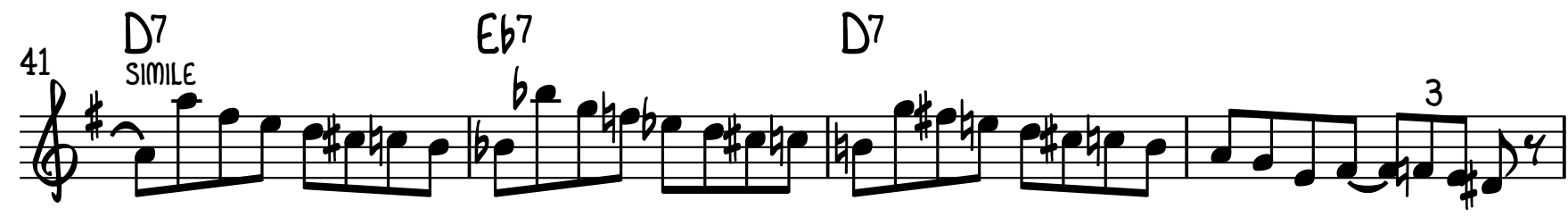

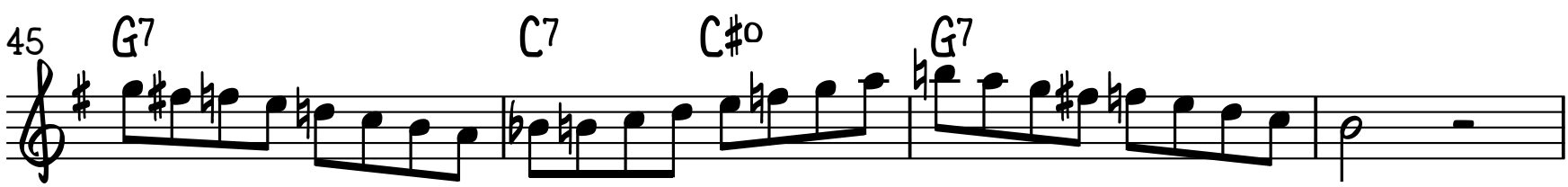

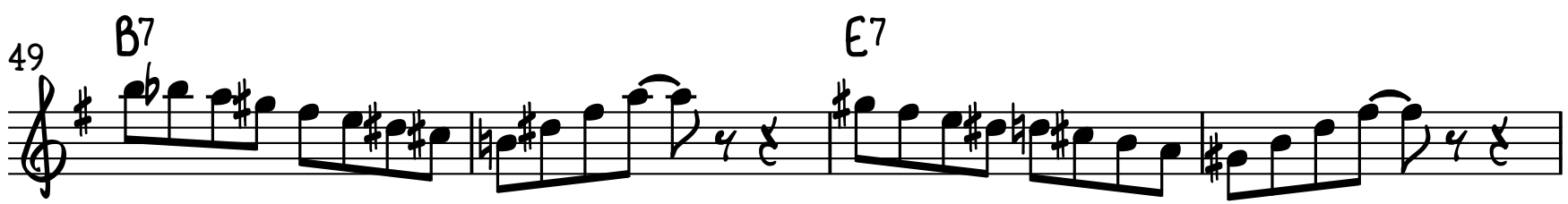

(4) ${ }^{27}$

$\begin{array}{llll}57 & D^{7} & E b 7 & D^{7}\end{array}$

(4)

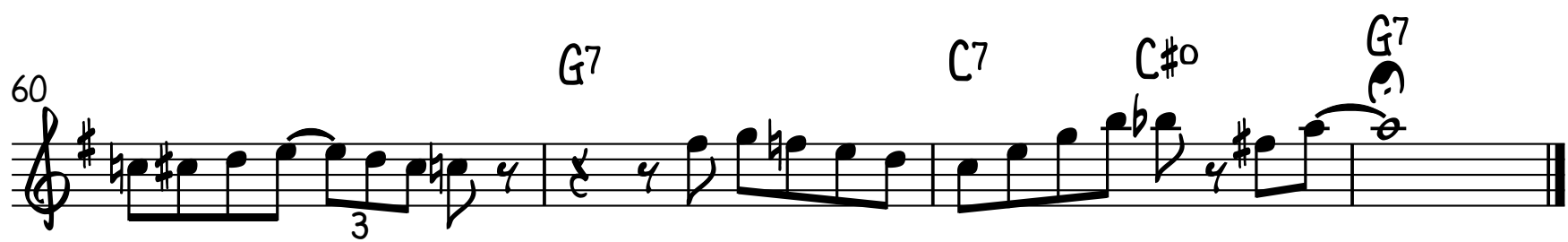


TRuer in ${ }^{\text {ES }}$ ETUDE 5 - MINOR BEBOP

TRACK $31-100$ BPM TRACK 32 - 120 BPM
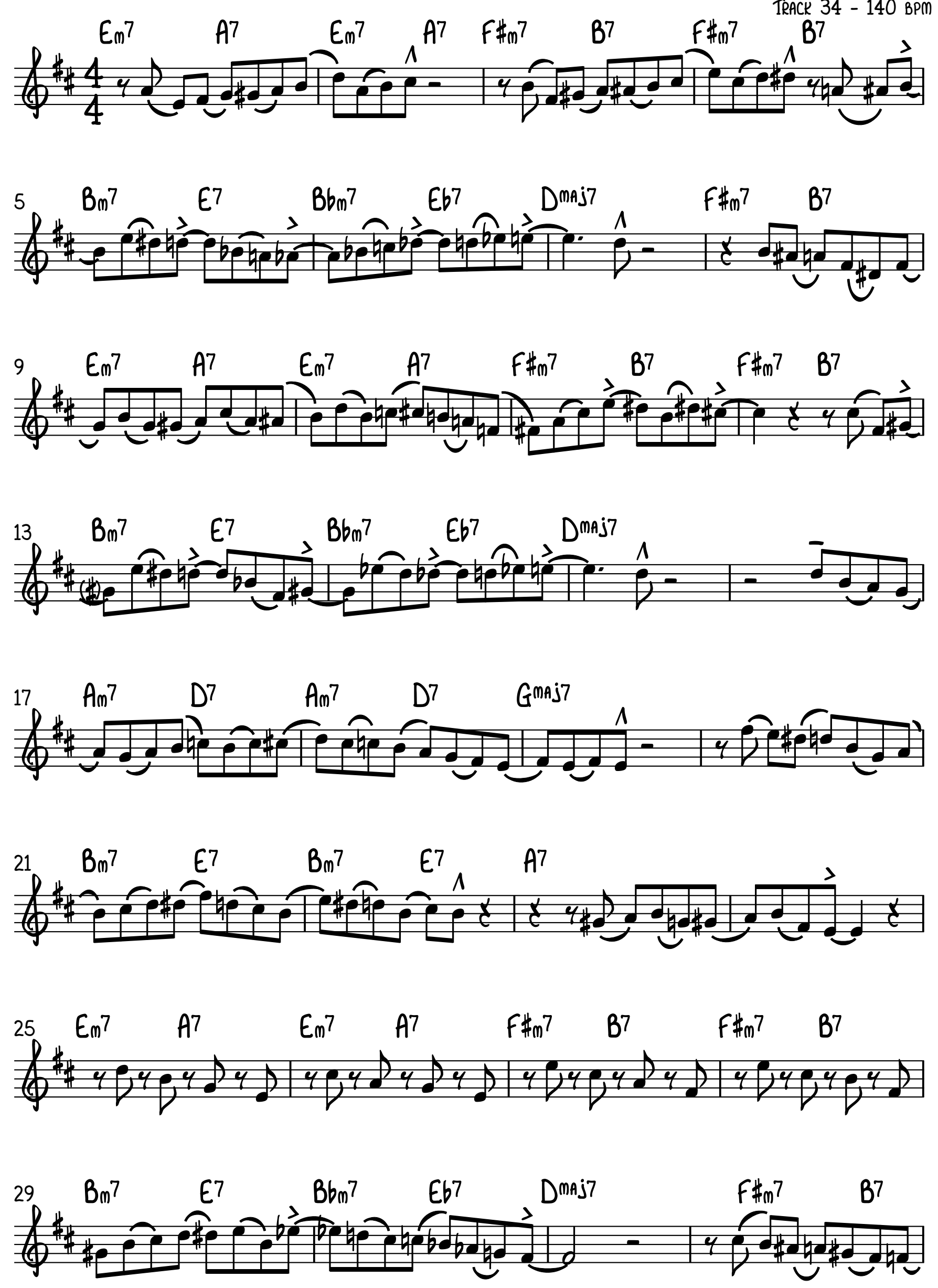
2
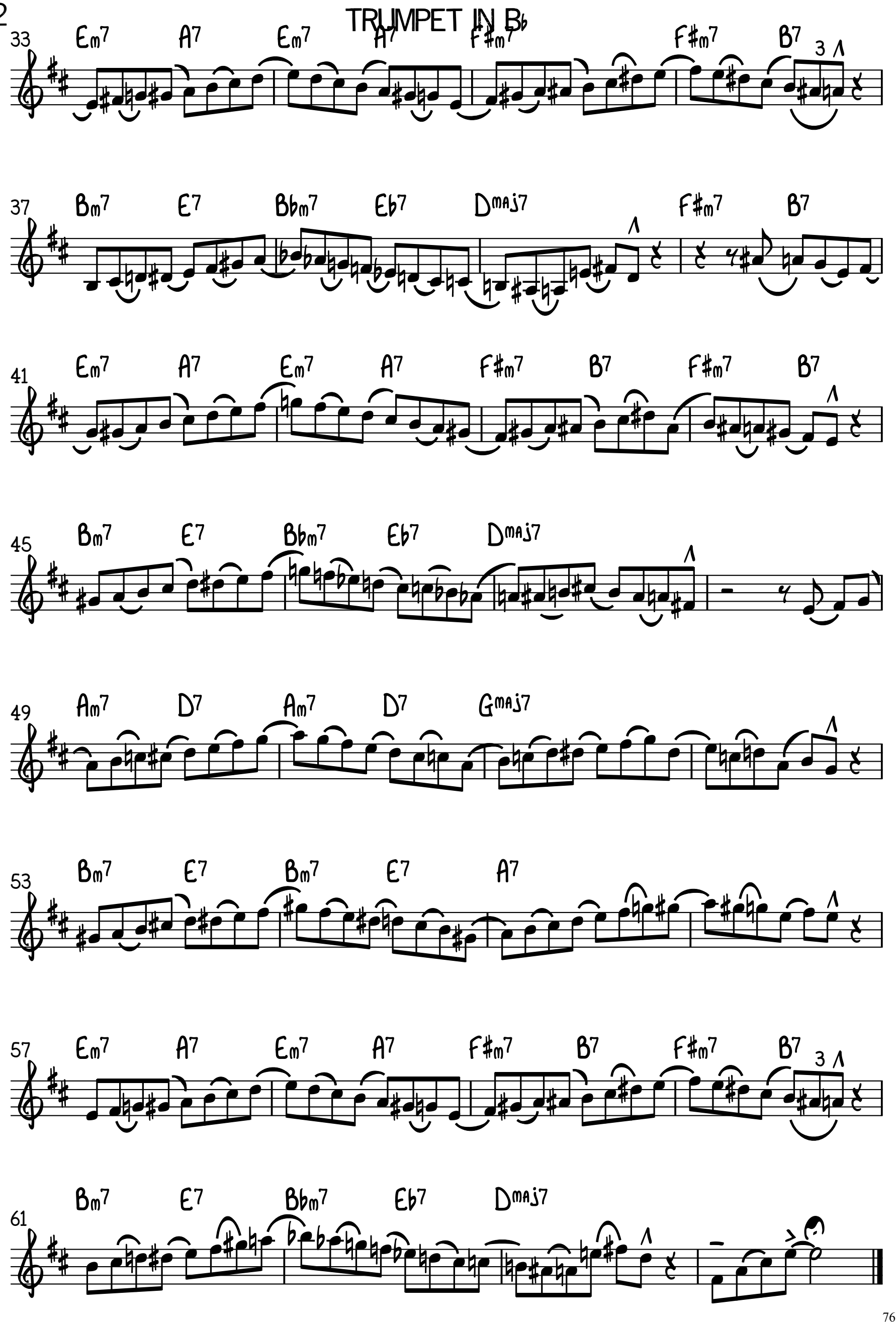
Exercises in Developing Bebop Scale Language

for Collegiate Jazz Students

\author{
Lars Swanson \\ West Virginia University
}

\author{
Tenor Sax Edition
}




\section{SCALE EXERCISE 1 - MAJOR BEBOP SCALES}

TRACK 1 - 100 BPM

TRACK 2 - $120 \mathrm{BPM}$

D ${ }^{\text {MAj7 }}$

TRACK 3 - 140 BPM

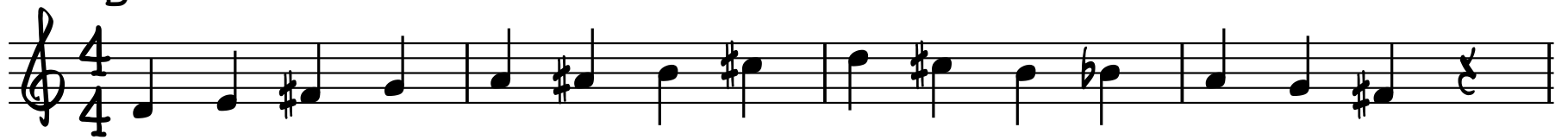

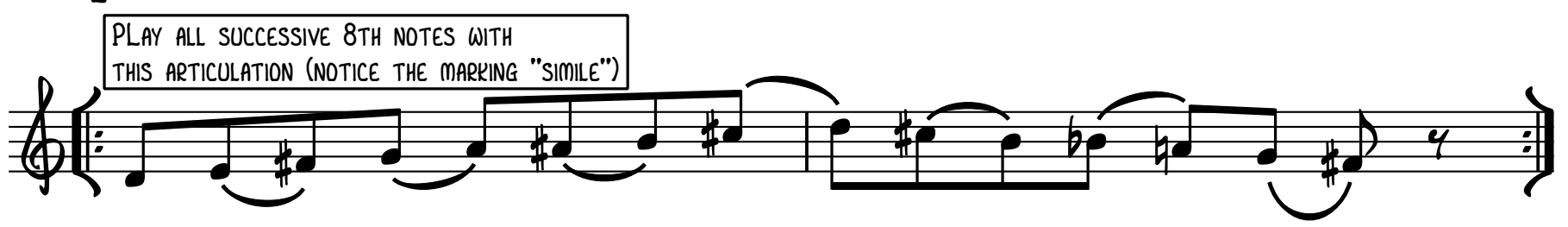

GMAj7

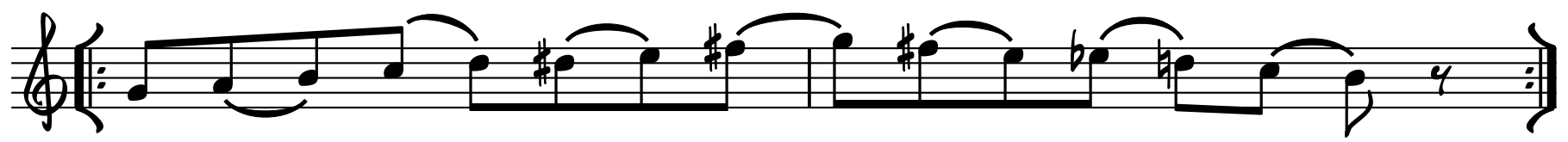

Cmaj7

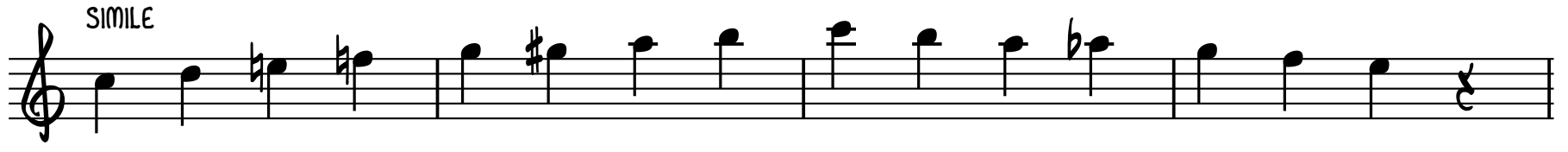

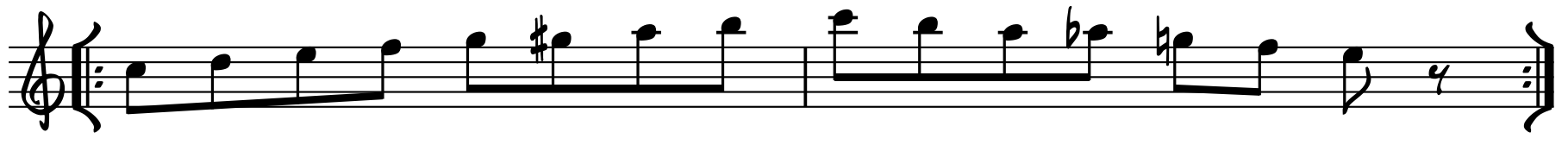

fmil $\ldots$

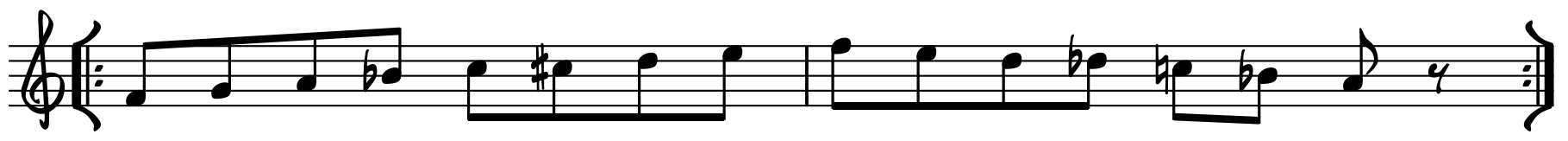

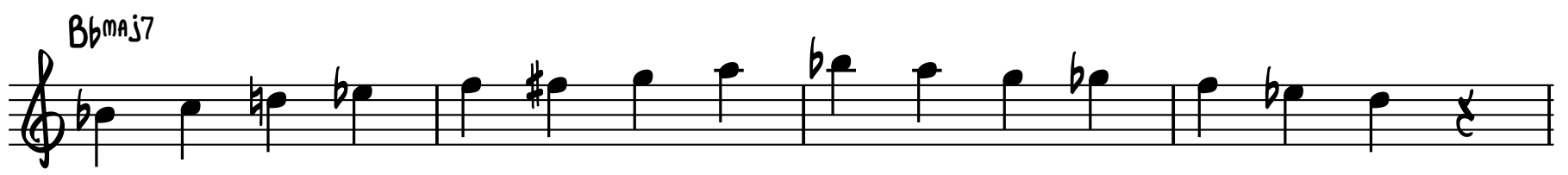




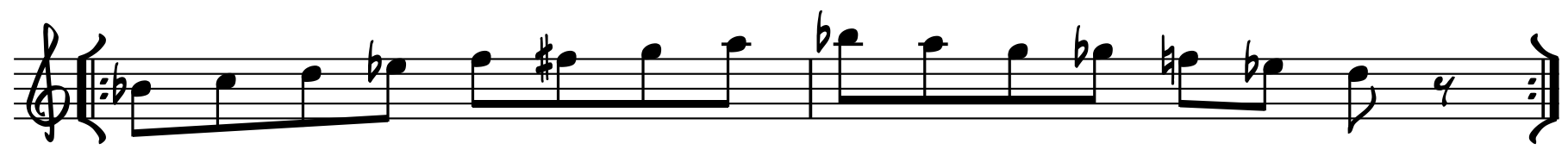

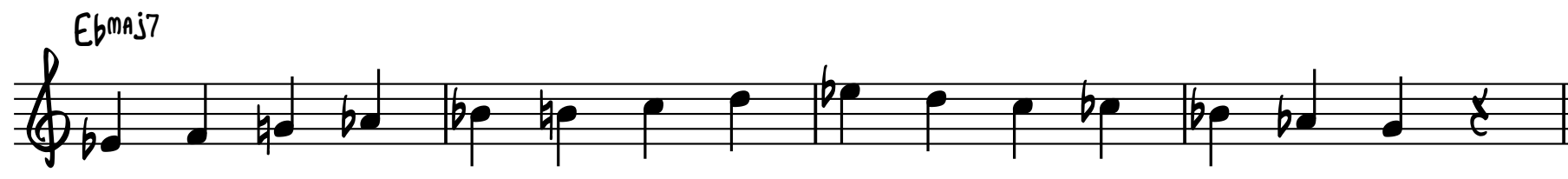

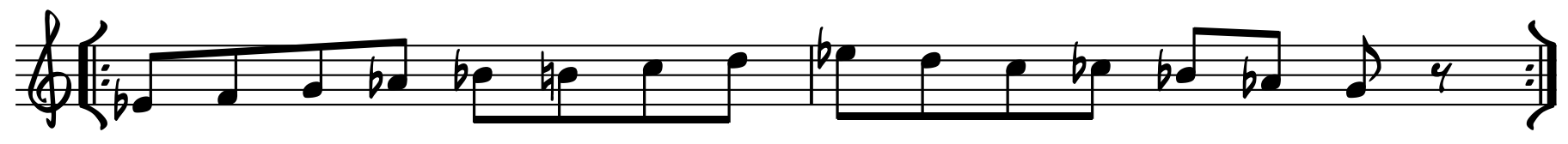

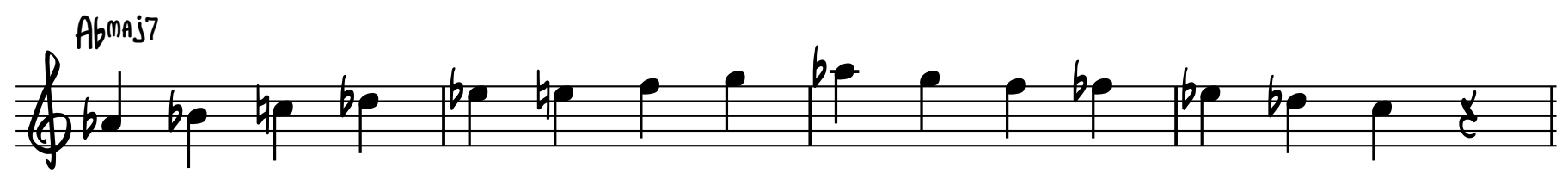

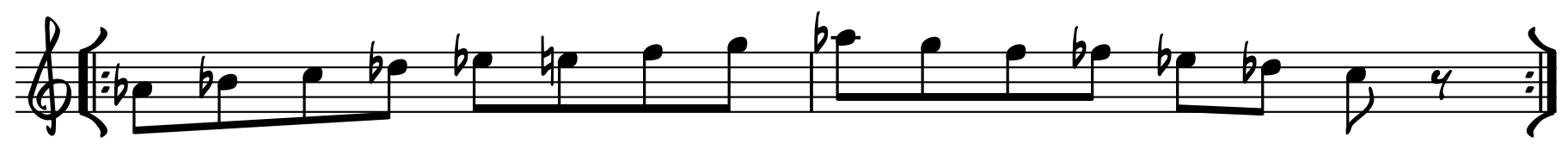

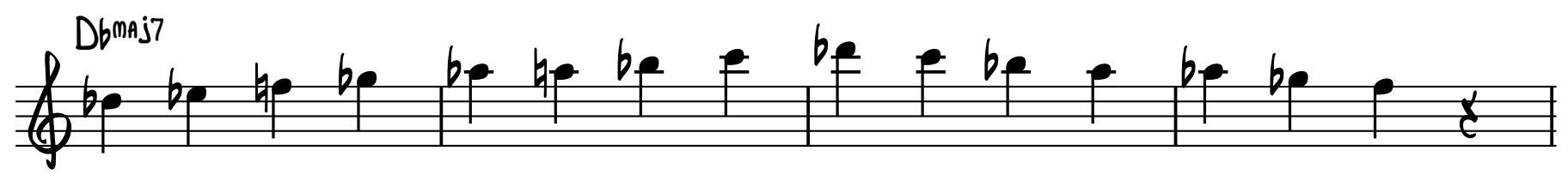

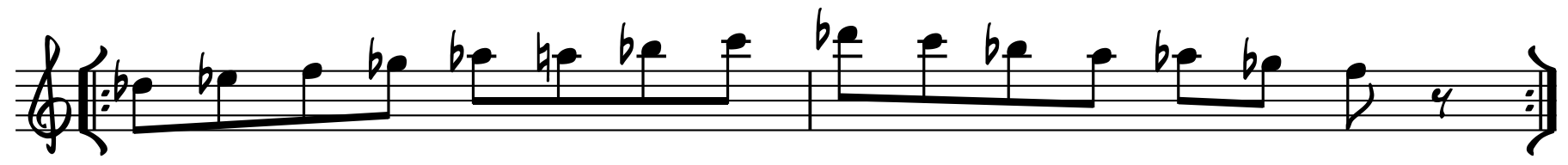

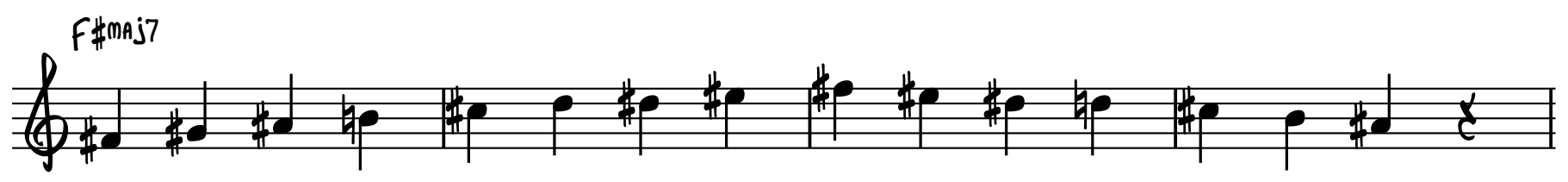

(4)

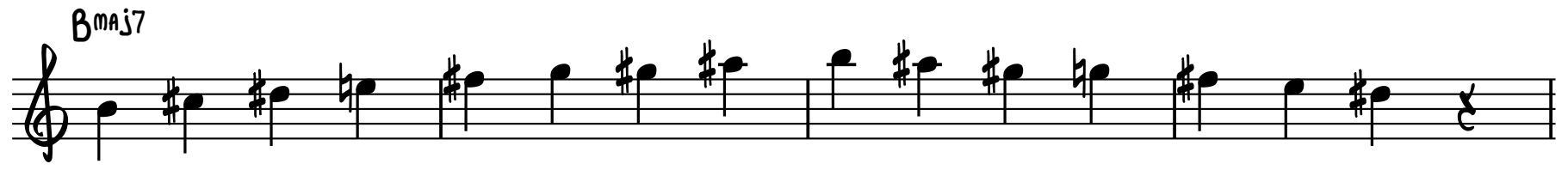



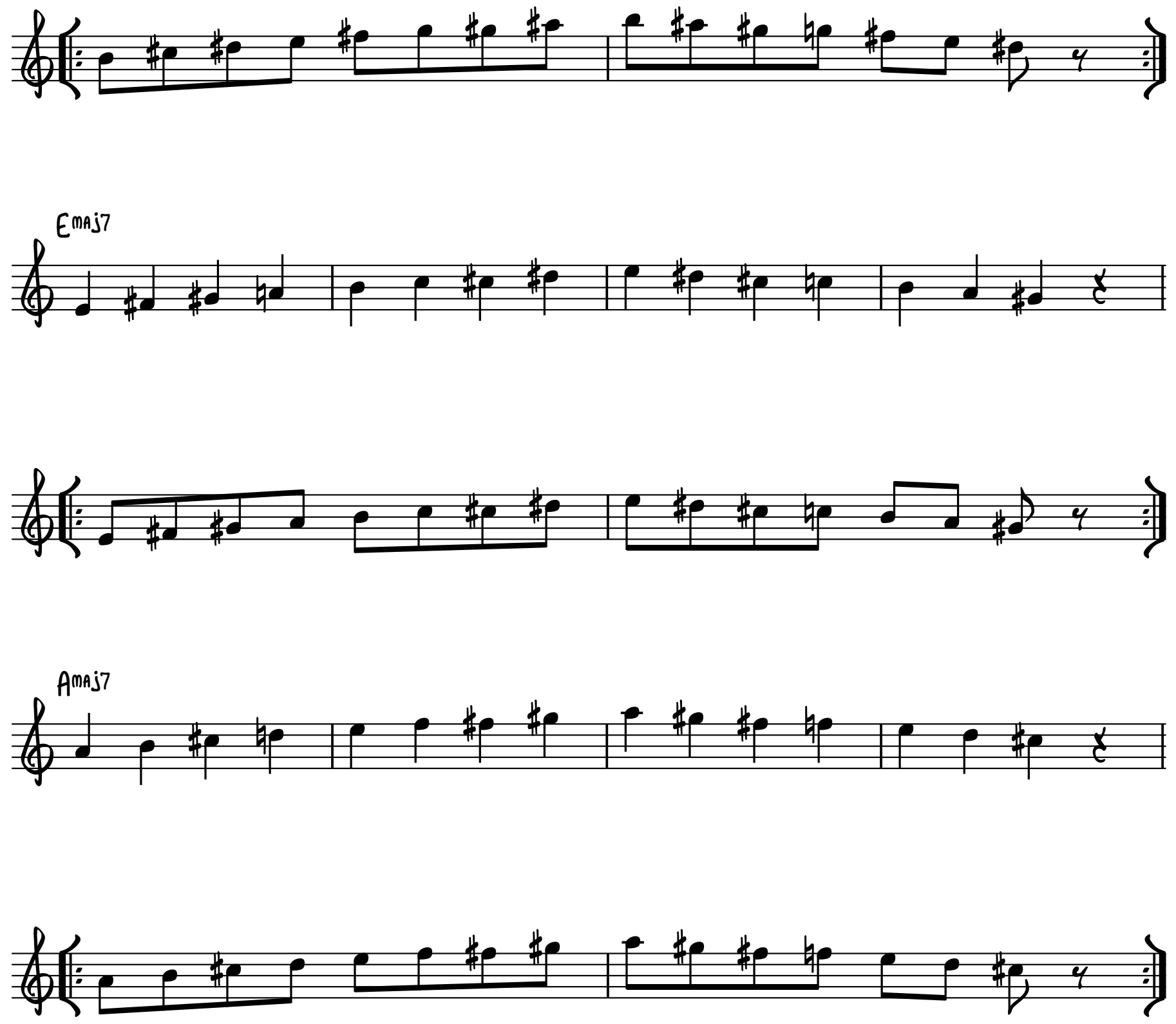

ONCE YOU HAVE MASTERED THIS AT THE FASTEST TEMPO, TRY TO PRACTICE THESE SCALES STARTING ON THE 3RD, 5TH AND 6TH SCALE DEGREES.

Cmaj7

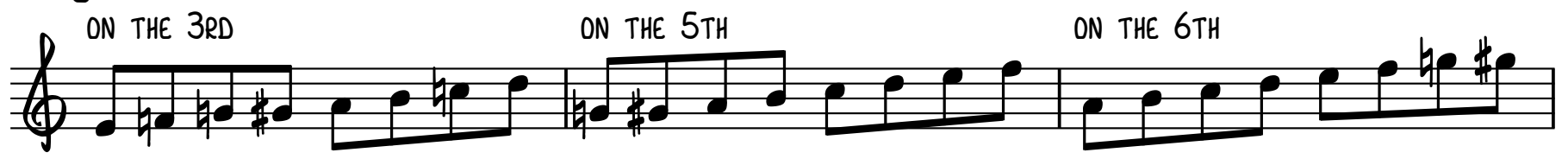

80 


\section{SCALE EXERCISE 2 - DOMINANT BEBOP SCALES}

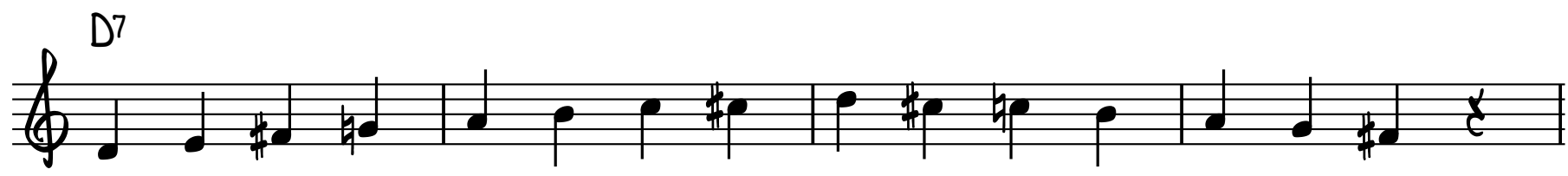

TRACK 10 - 100 BPM TRACK $11-120$ BPM TRACK 12 - 140 BPM
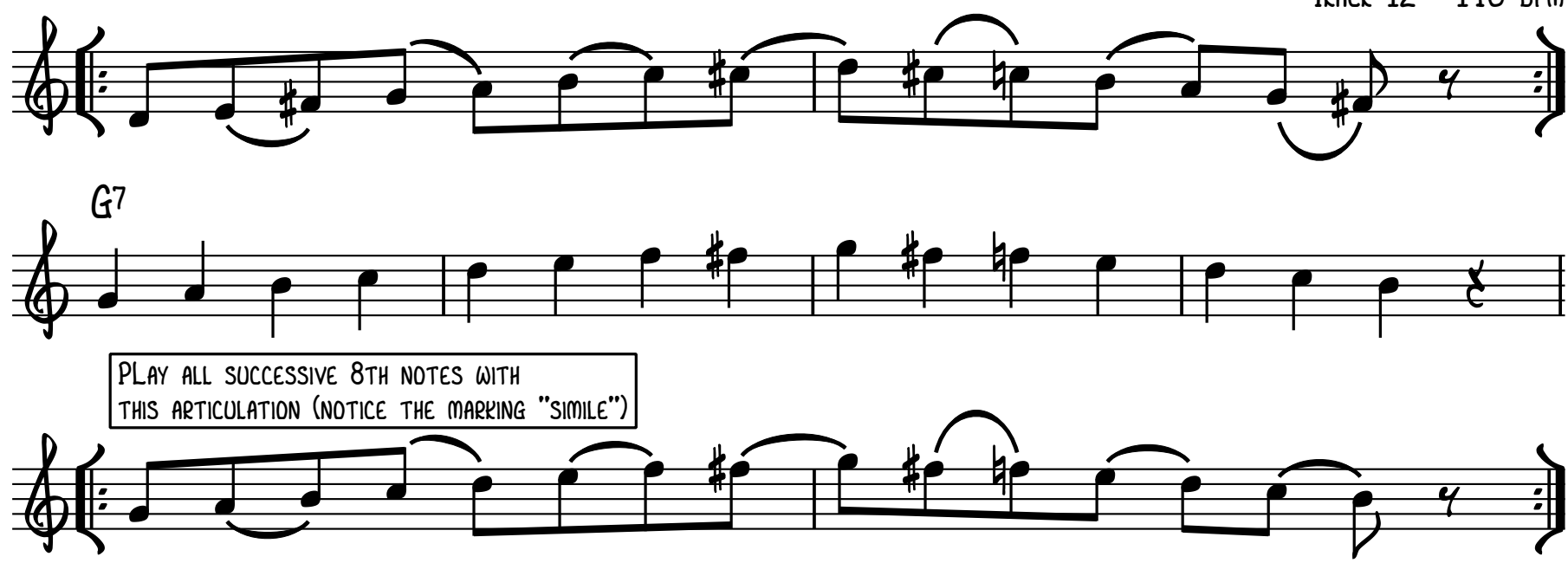

C7

$\oiint^{\text {SIMILE }}$
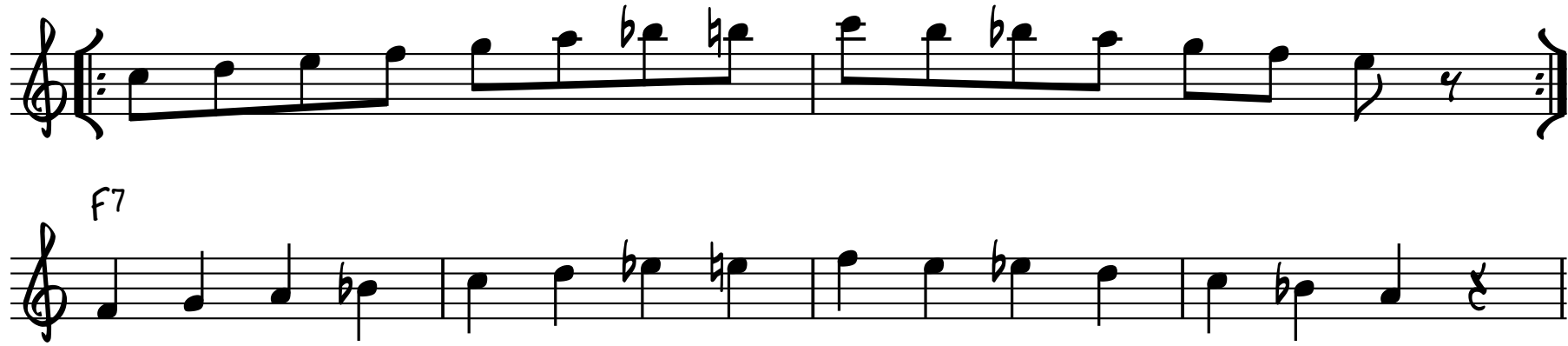

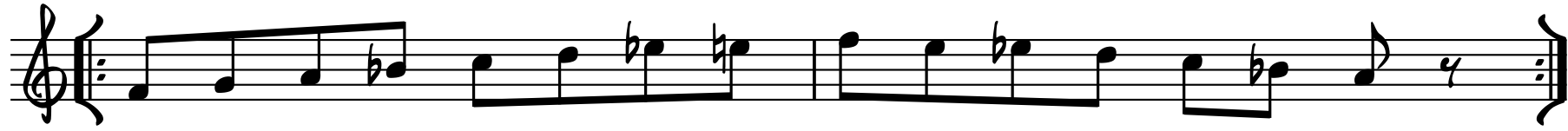

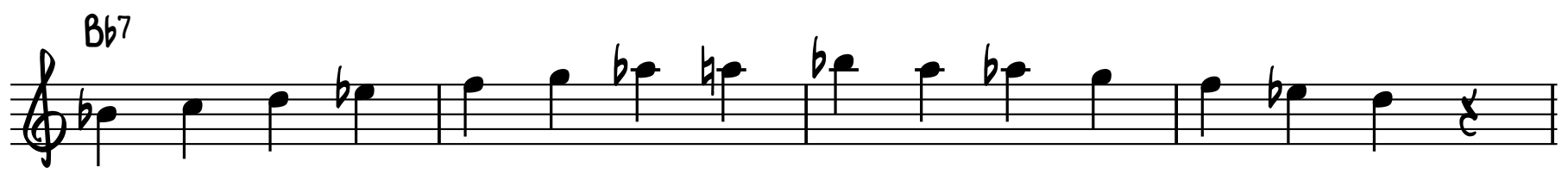

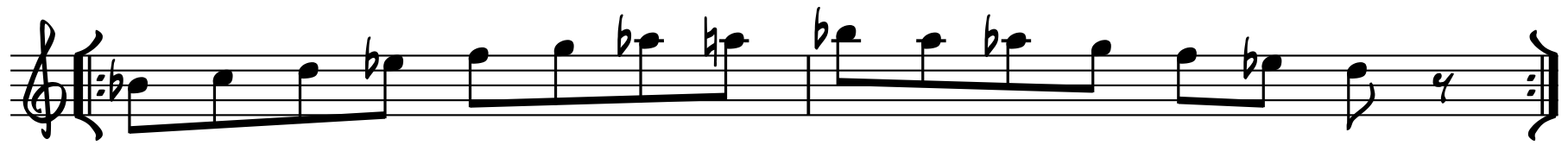




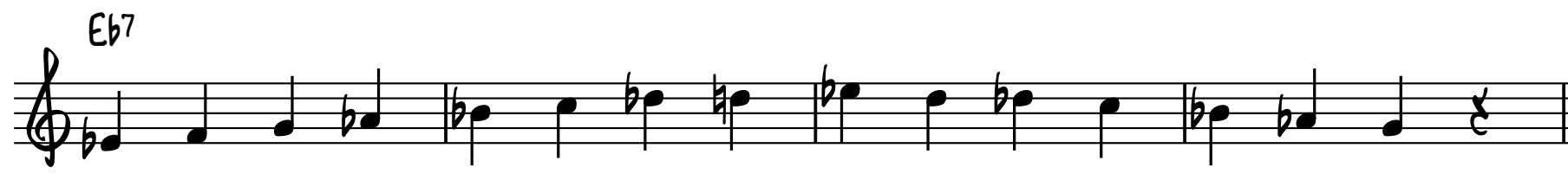

费( Abt

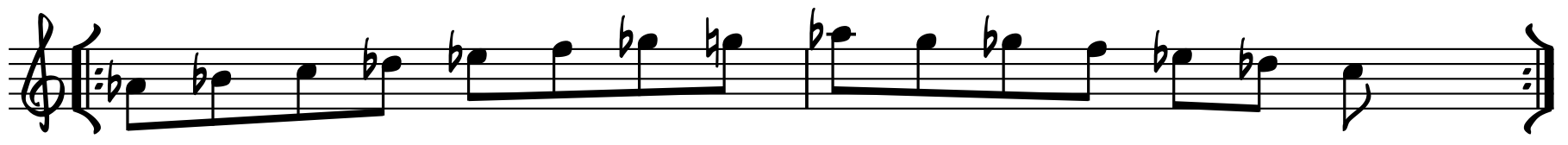
Domis

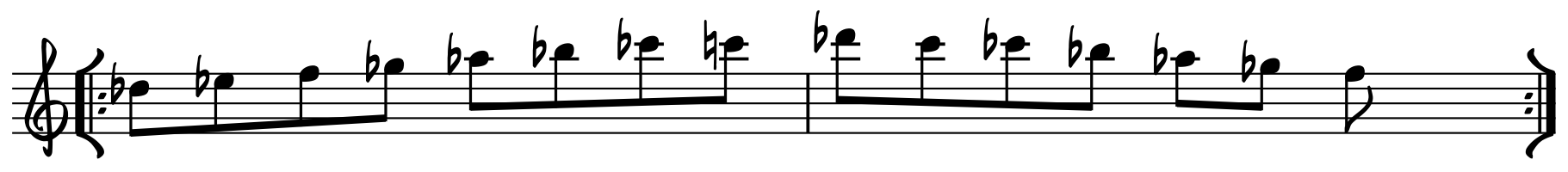

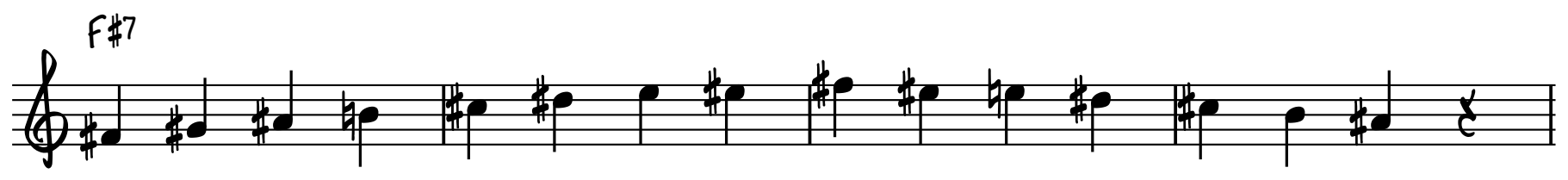

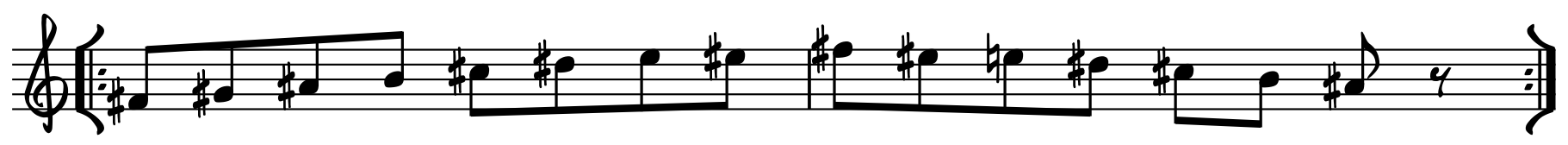

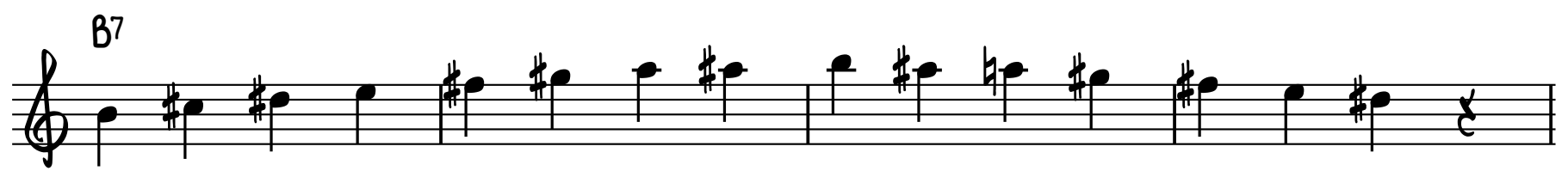

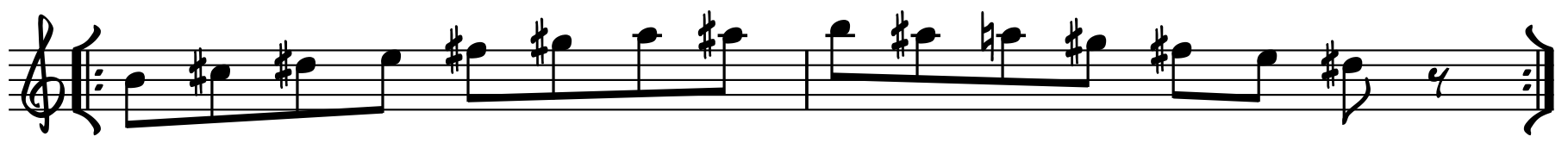



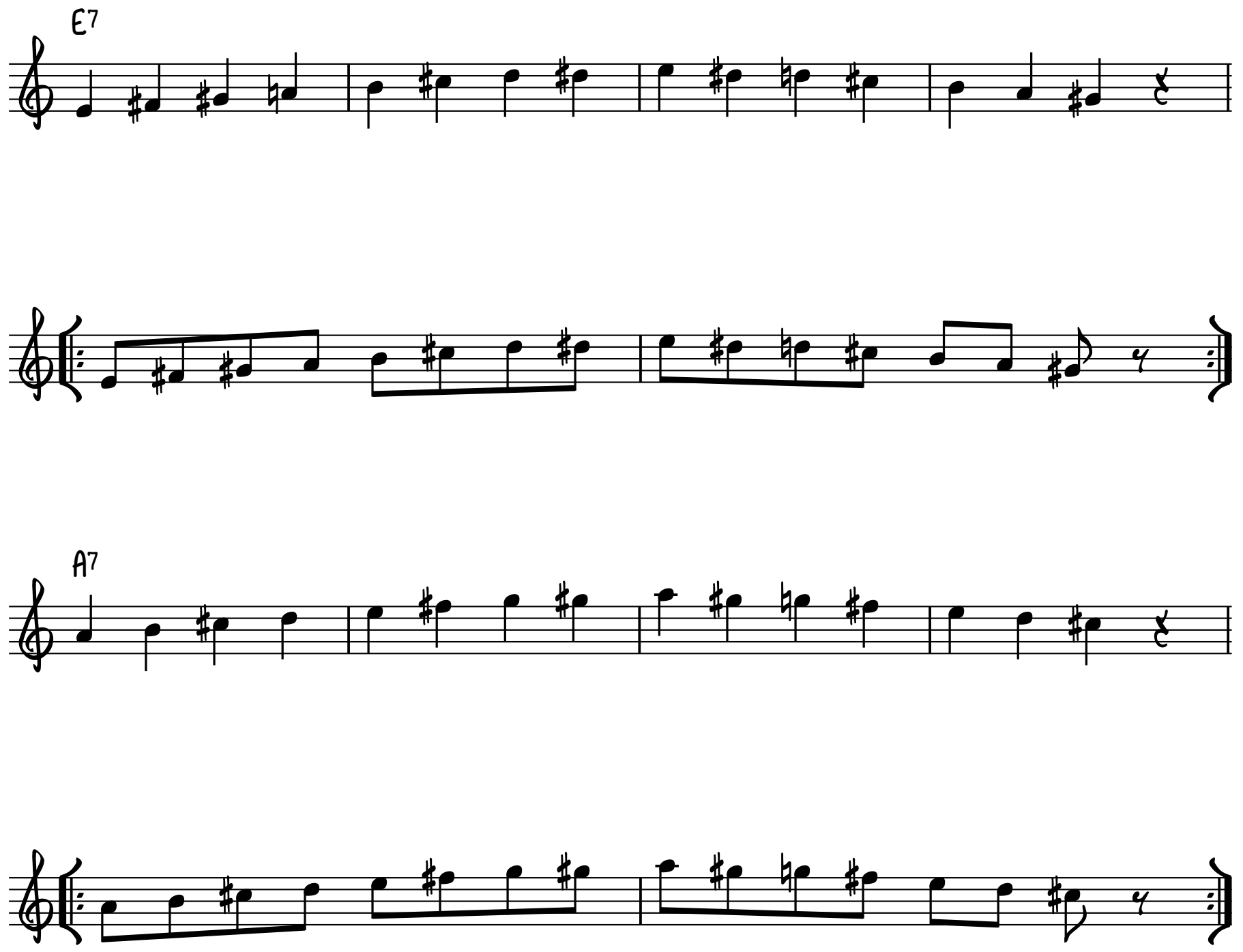

ONCE YOU HAVE MASTERED THIS AT THE FASTEST TEMPO, TRY TO PRACTICE THESE SCALES STARTING ON THE 3RD, 5TH AND FLAT 7TH SCALE DEGREES.

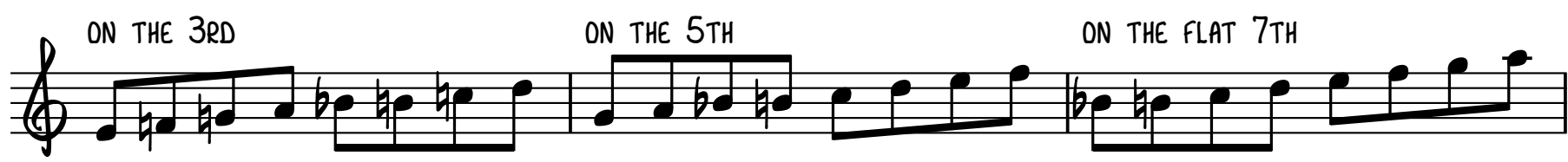




\section{SCALE EXERCISE 3 - MINOR BEBOP SCALES}

TRACK $19-112$ BPM TRACK $20-132 \mathrm{BPM}$

TRACK 21 - 152 BPM

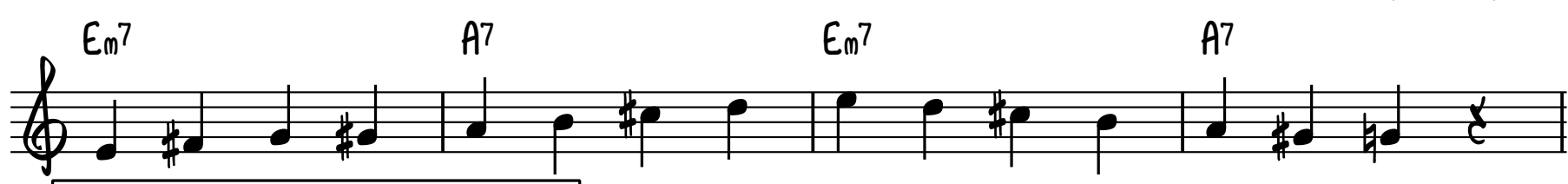

PLAY ALL SUCCESSIVE 8TH NOTES WITH

THIS ARTICULATION (NOTICE THE MARKING "SIMILE")
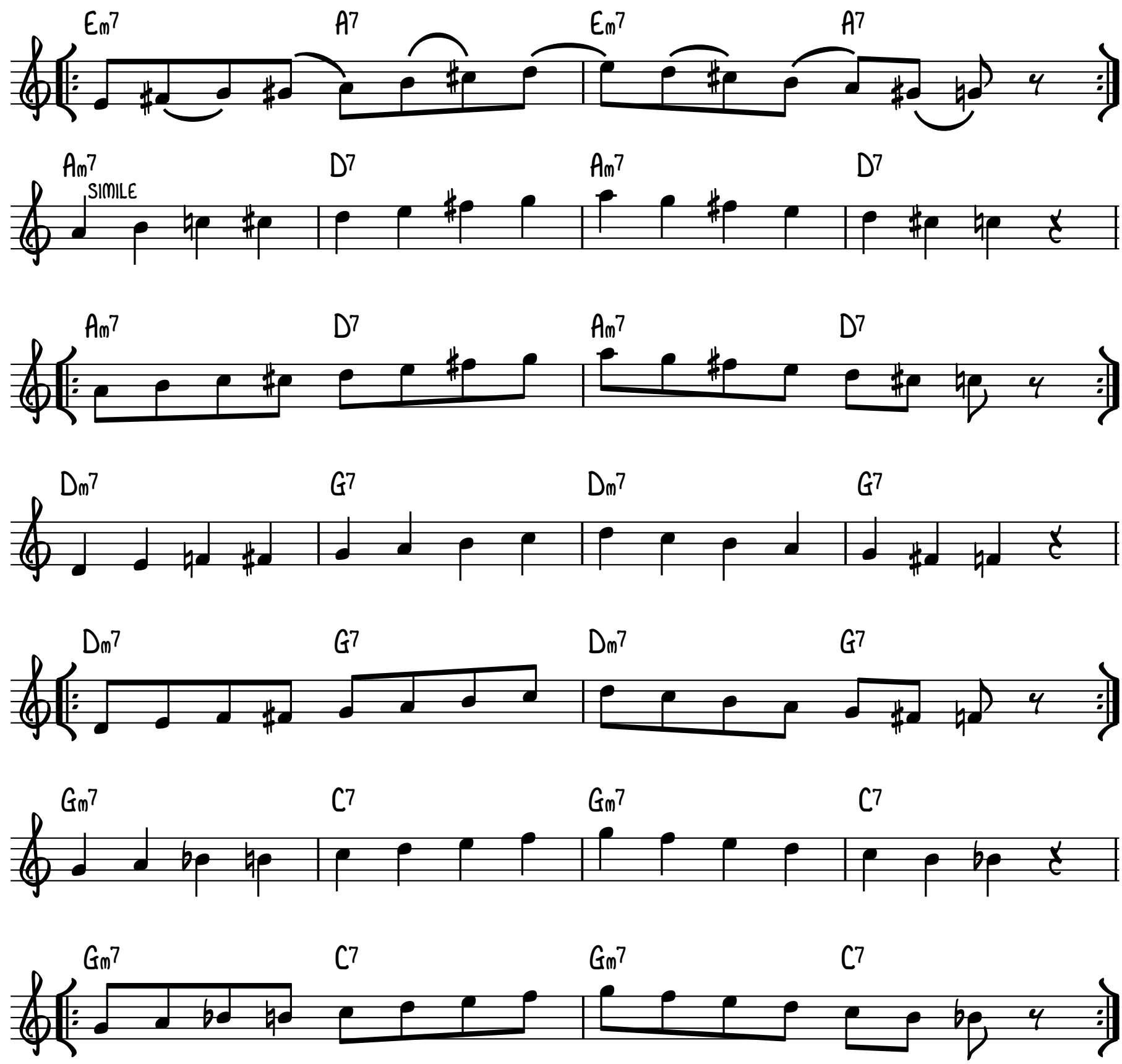


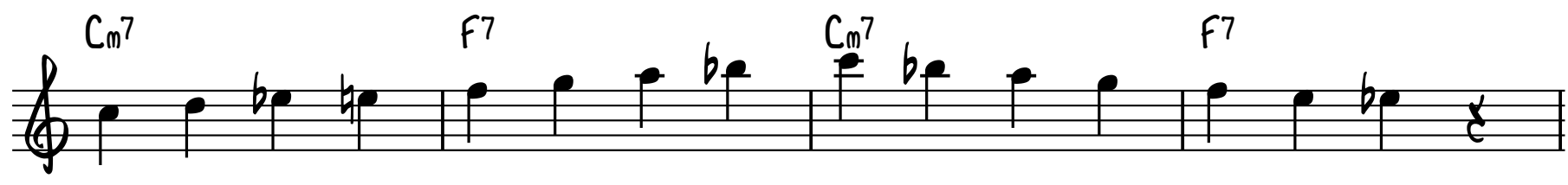

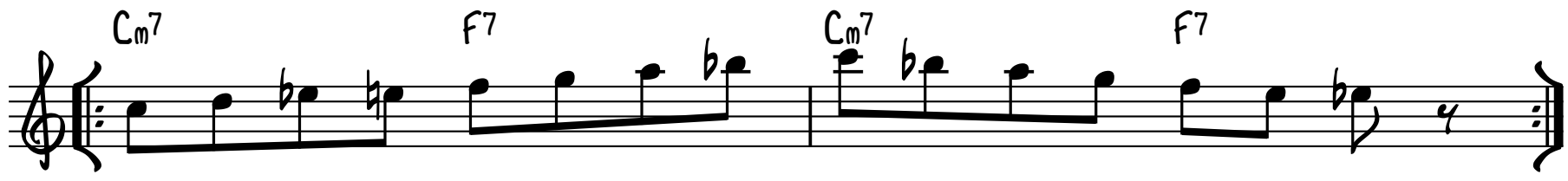

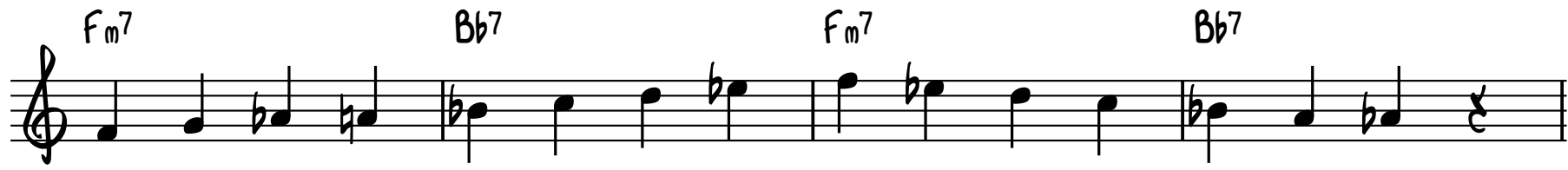

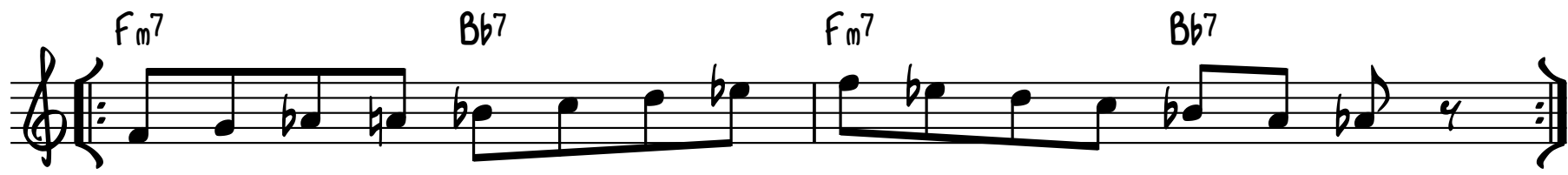

$b_{b \%}^{B b_{n} 7}$

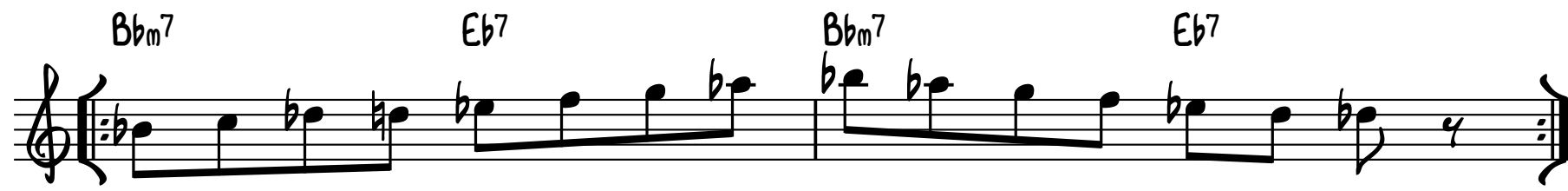

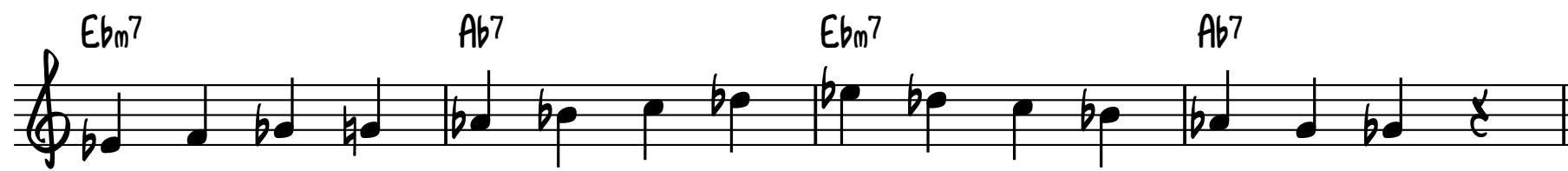

船(

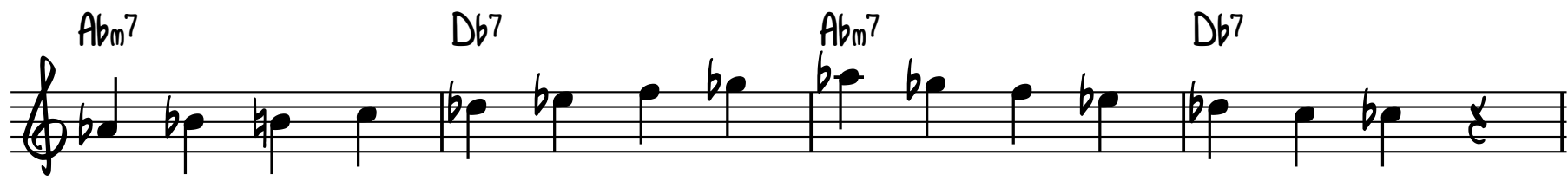

触 
W
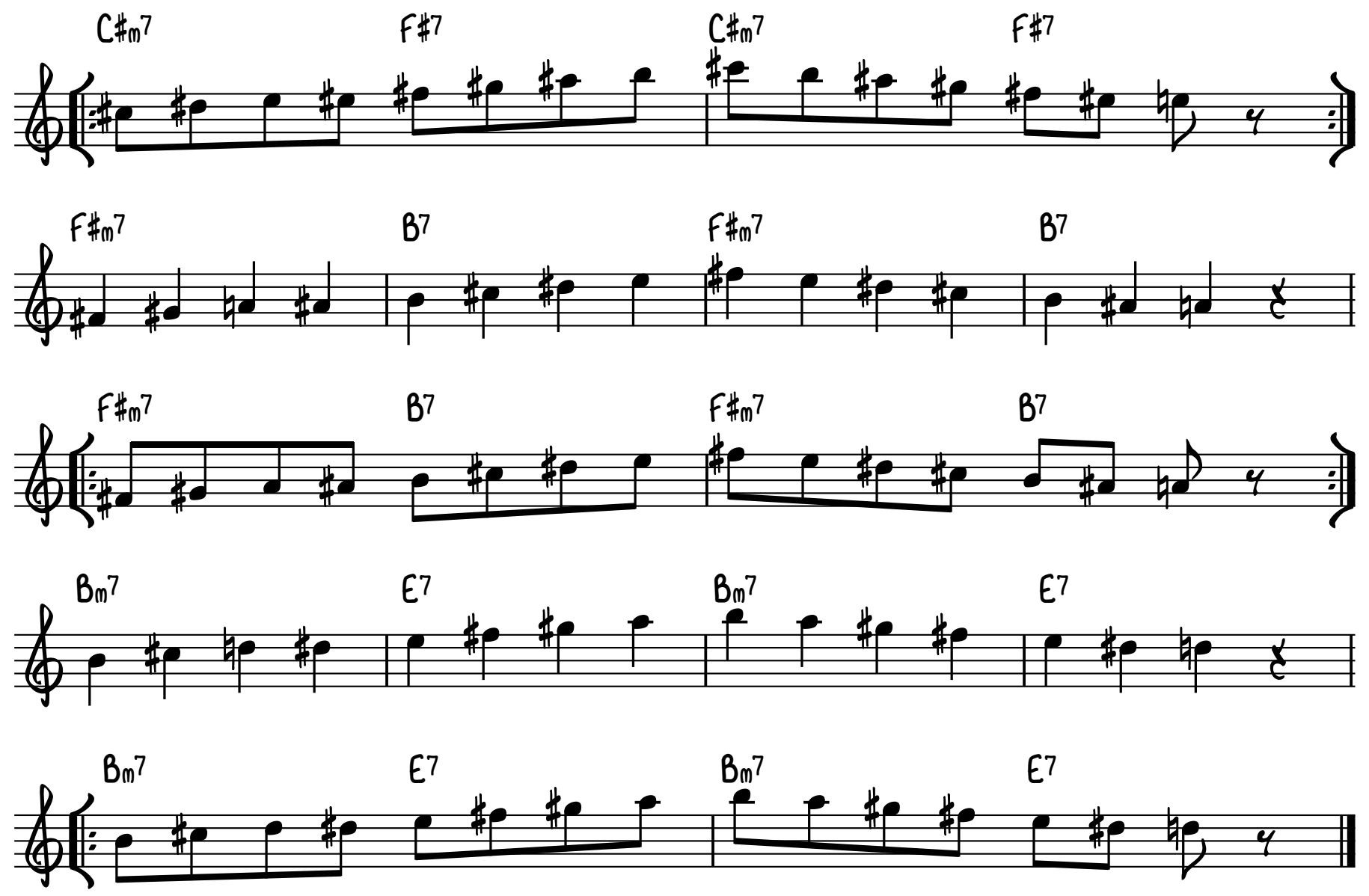

THIS IS THE 5TH MODE OF THE DOMINANT BEBOP SCALE. FOR FURTHER EXPLORATION, REFER TO THE EXPANSION IN SCALE EXERCISE 2. 
$\mathrm{Gm} 7$

C7

Fmaj7 TraCK $23-120$ BPM

$\oiint_{4}^{4}$

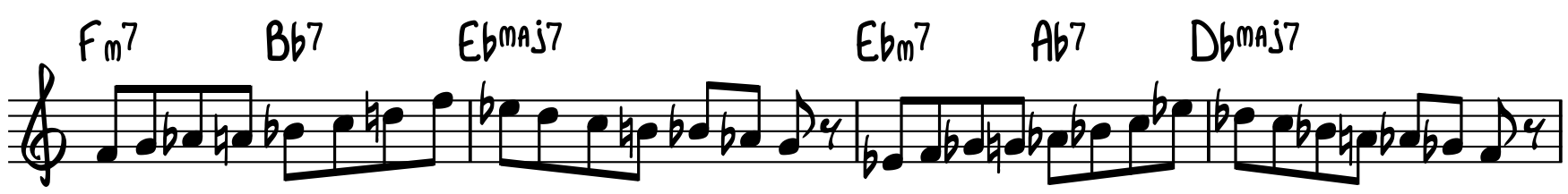

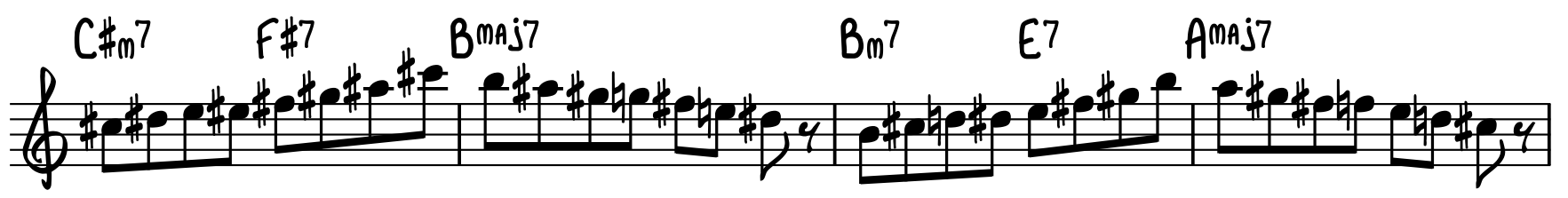

$\mathrm{C}_{m} 7$

F7

Bbmaj7

$\mathrm{Bbm}^{7}$

Eb7

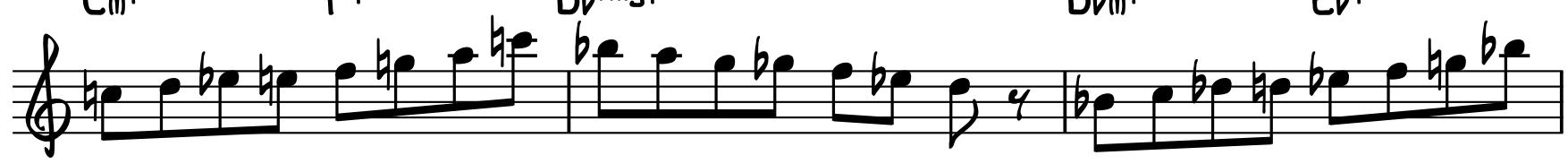

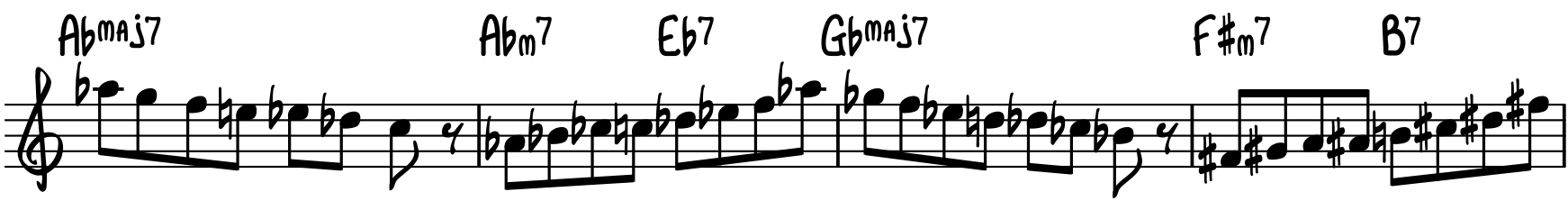
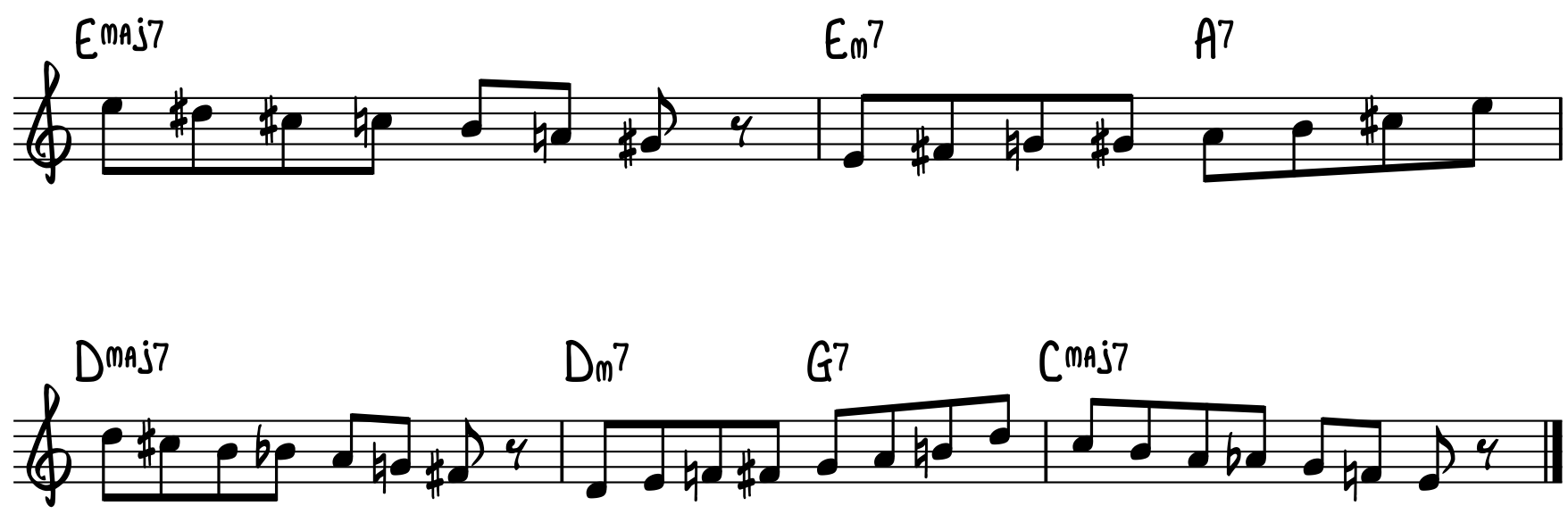


\section{TENOR SAXOPHONE \\ TWO BAR II-V}

TRACK 25 - 100 BPM TRACK $26-120$ BPM

$\underbrace{\mathrm{Bm}^{7}}$

(4)

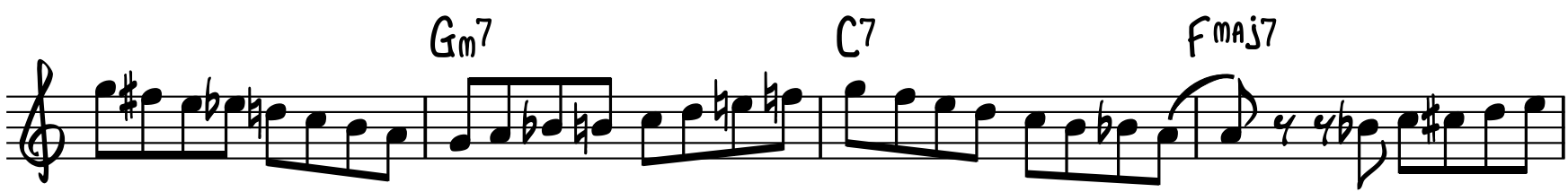

$\begin{array}{lll}\mathrm{Fm}_{m} 7 & B b 7 & \text { Ebmaj7 }\end{array}$

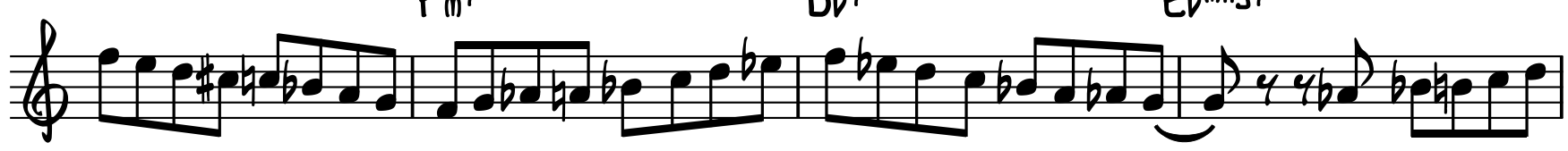

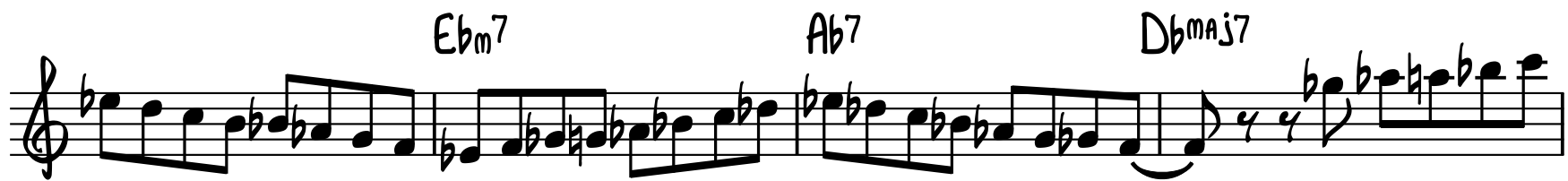

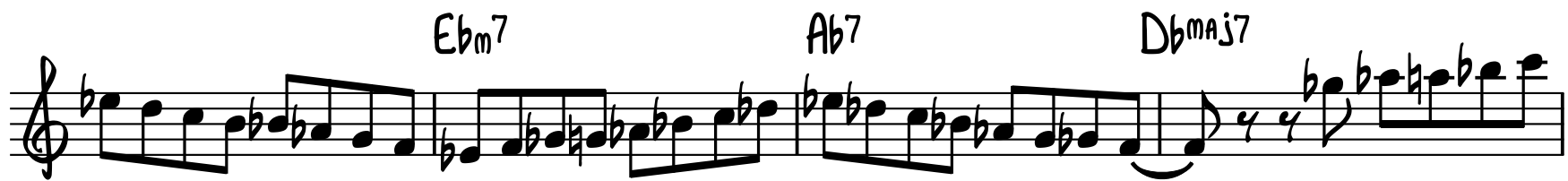

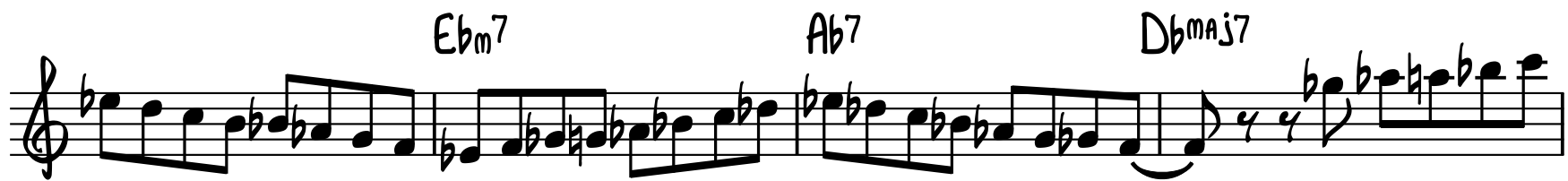

C $\$ m 7$

$f^{b e}$ ebe bebe be

$\int^{B \text { MAj7 }}$

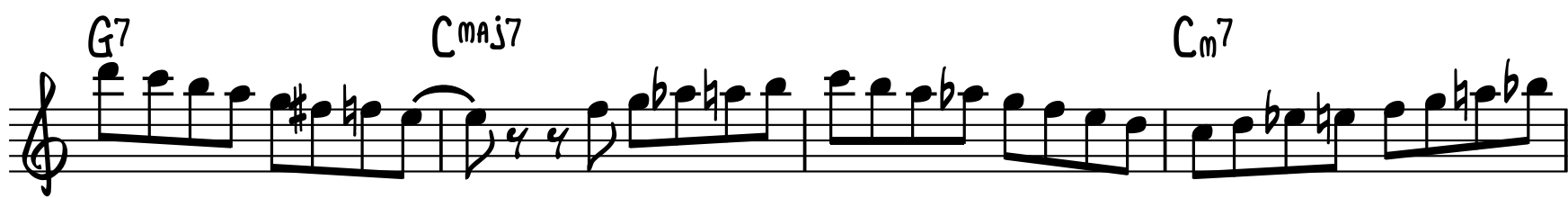




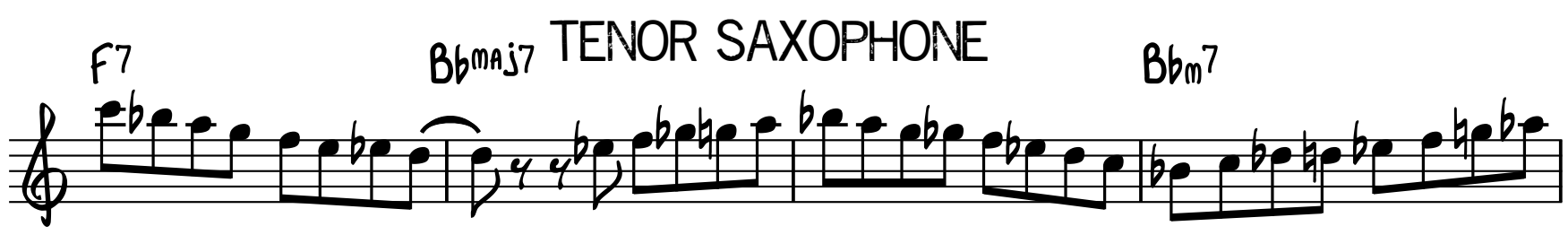

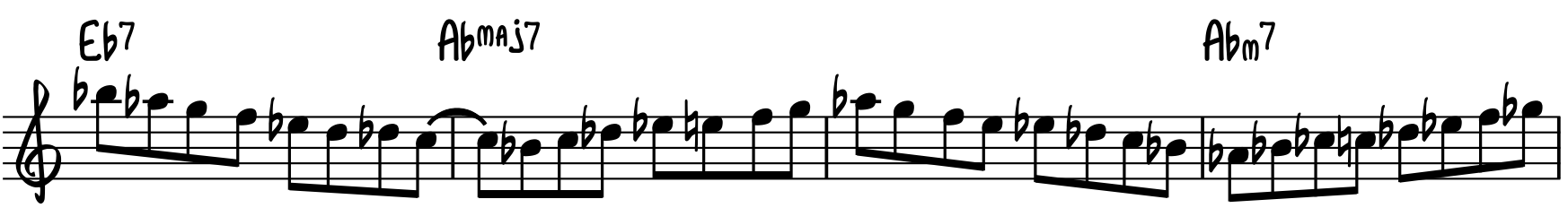

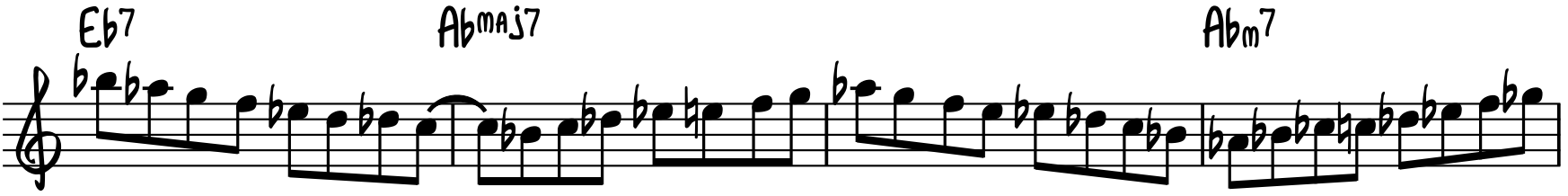

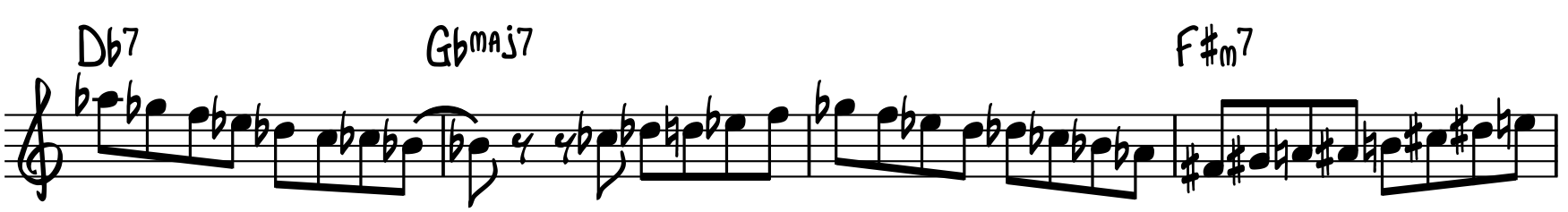

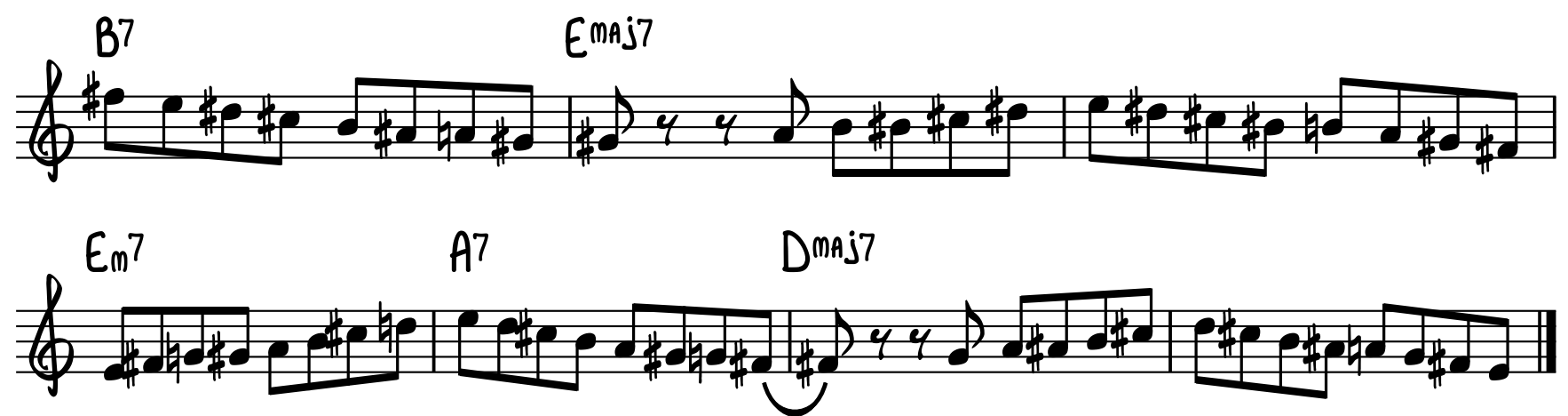




\section{TENOR SAXOPHONE \\ I TO IV MODULATION THROUGH II-V}

TRACK 28 - 92 BPM

TRACK 29 - 112 BPM

TRACK 30 - 132 BPM

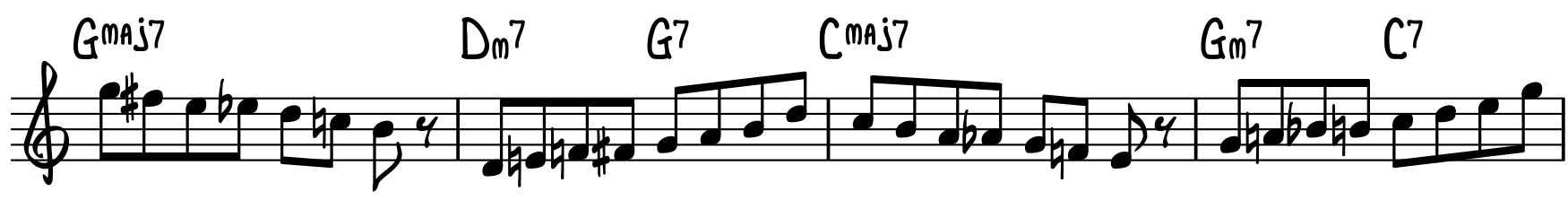
Fmaj7
$\mathrm{Cm}^{7}$
F7
Bbmaj7
$\mathrm{Fm}^{7}$
Bb7

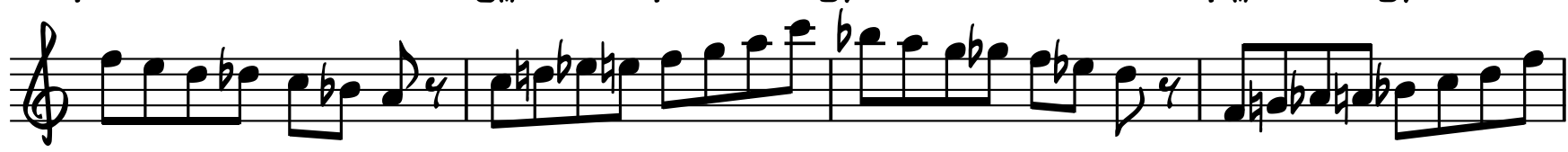

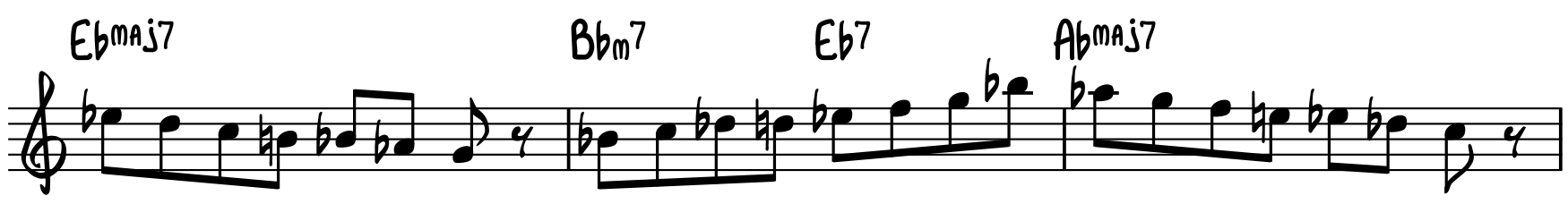

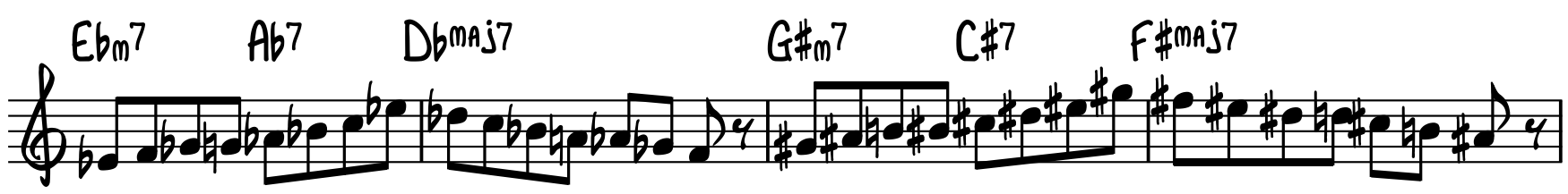

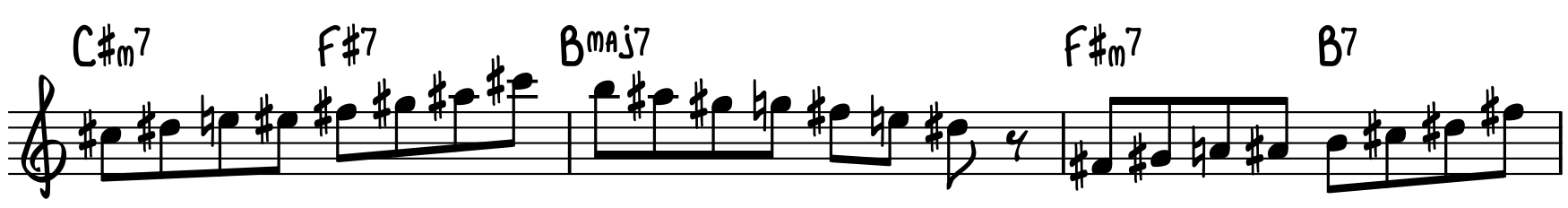

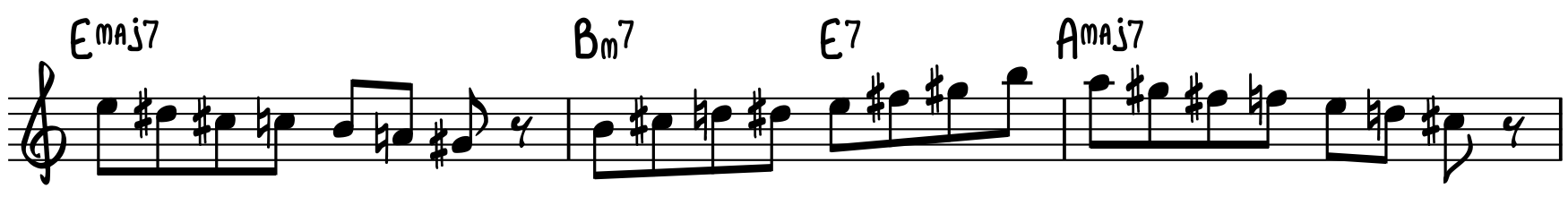

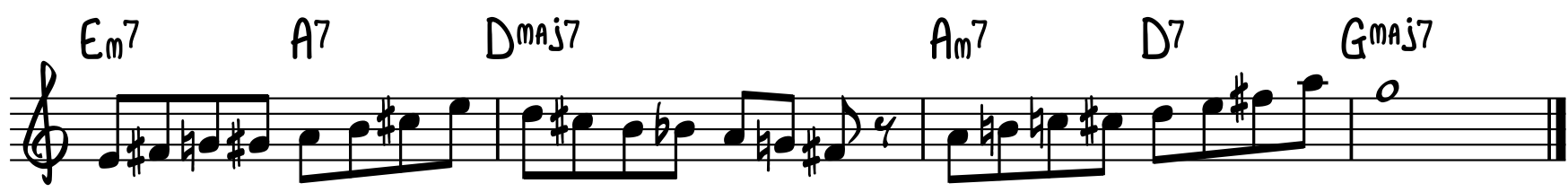


TENOR SAXOPHONE

EVEN 8THS

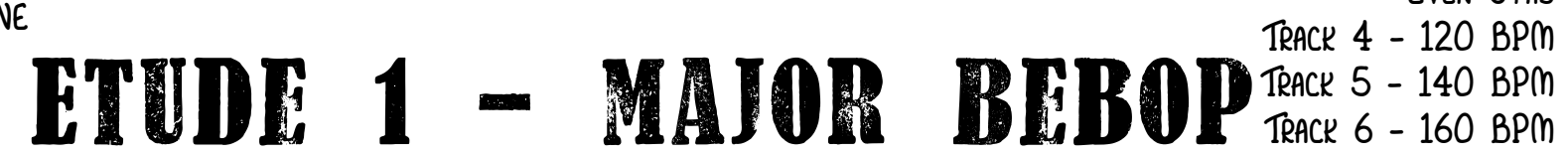
$\frac{1}{4} 4$ ant

$5 \quad G^{\text {maj7 }}$

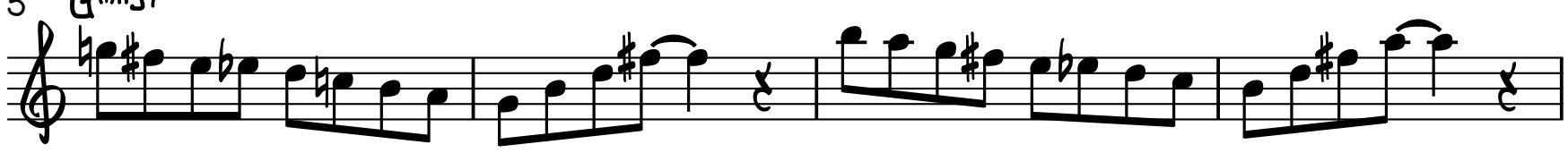

9 Fmaj7

(4)

13 Gmaj7

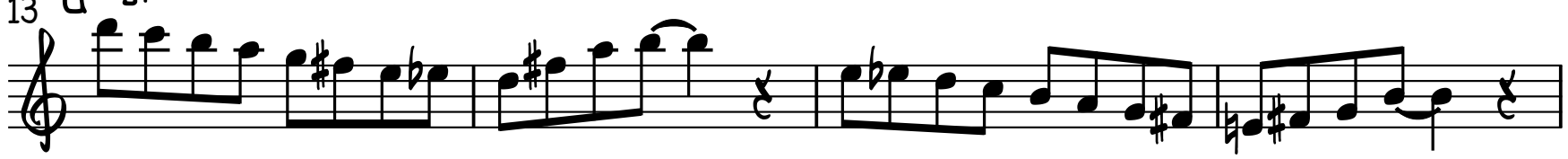

$17 \quad A^{\text {maj7 }} \quad$ DMAj7

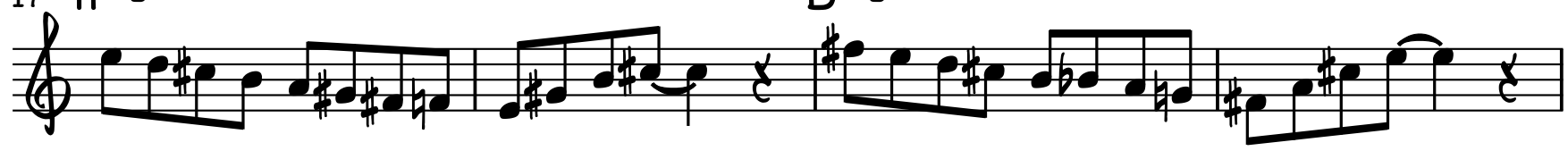

${ }_{21}$ Gmaj7 Cmaj7

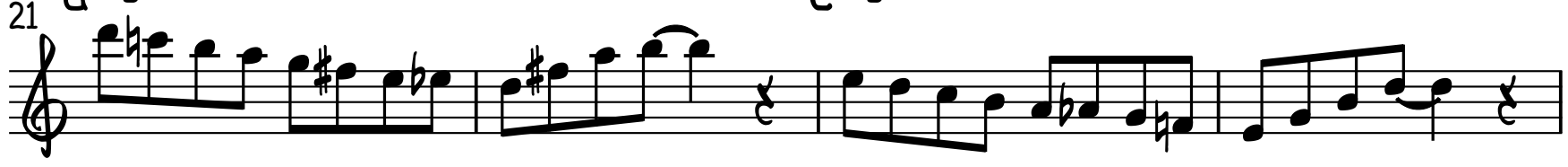

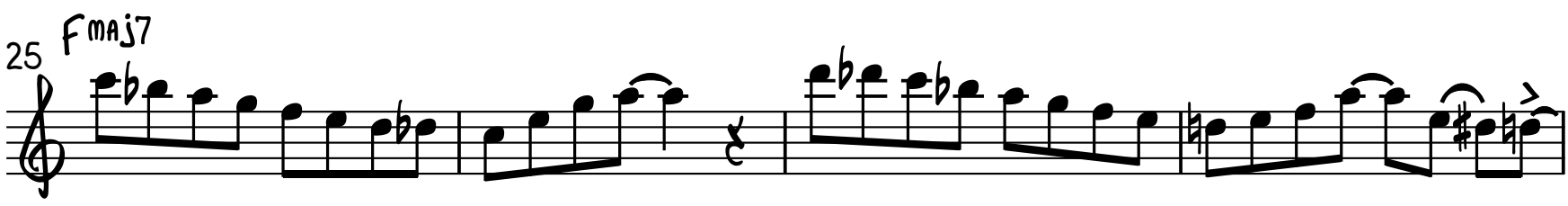

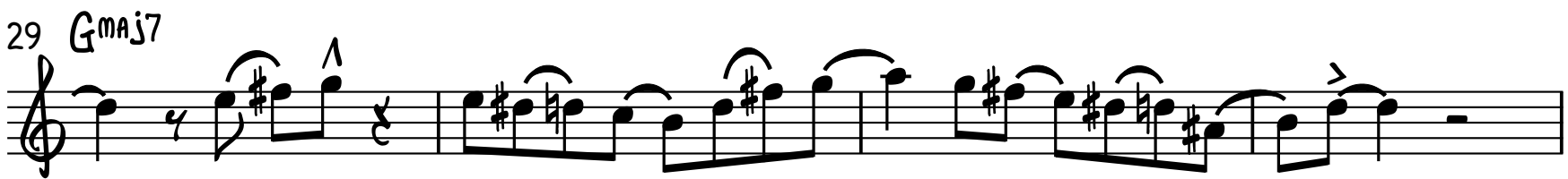


2 TENOR SAXOPHONE (18)

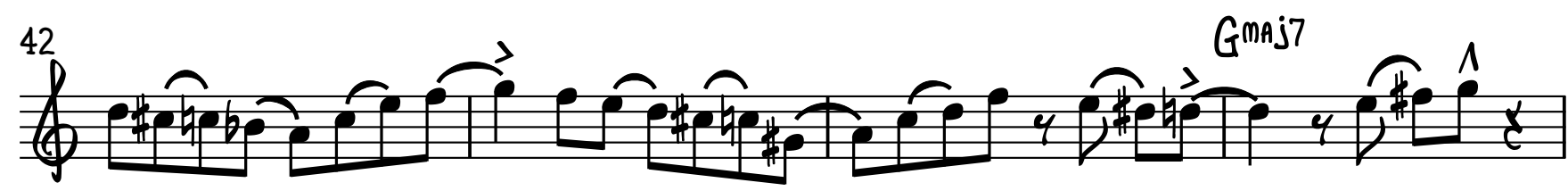

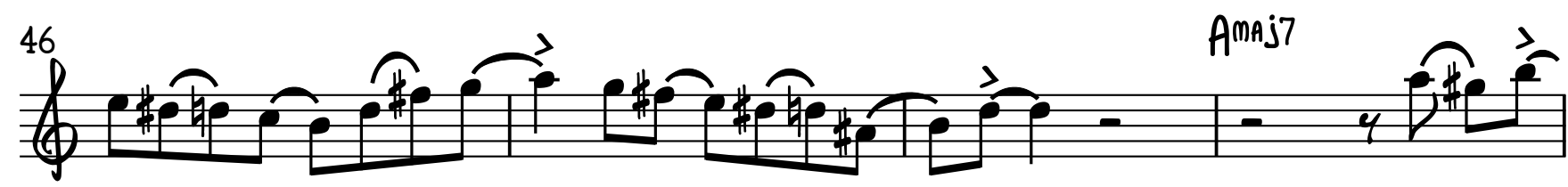

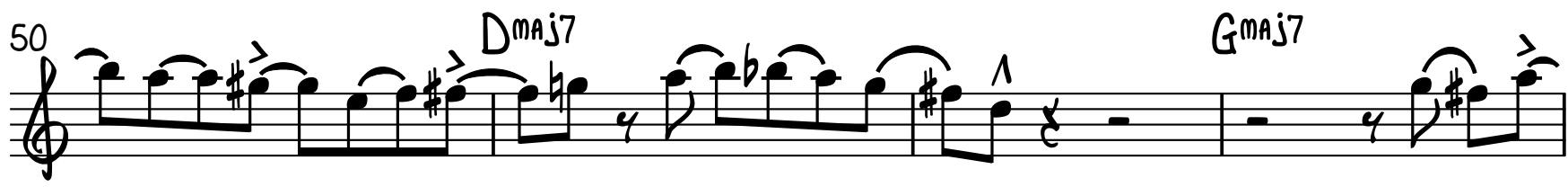

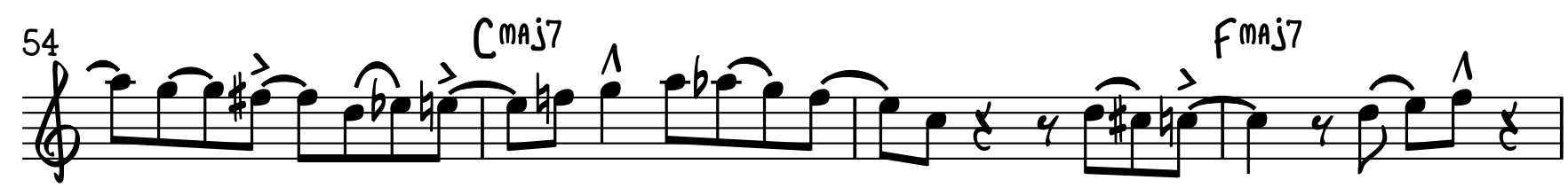

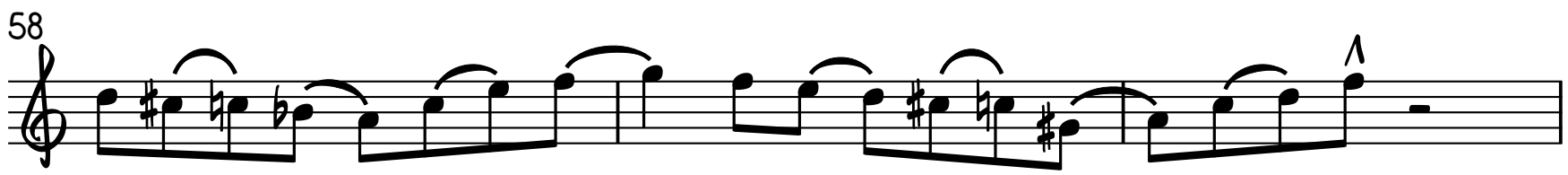

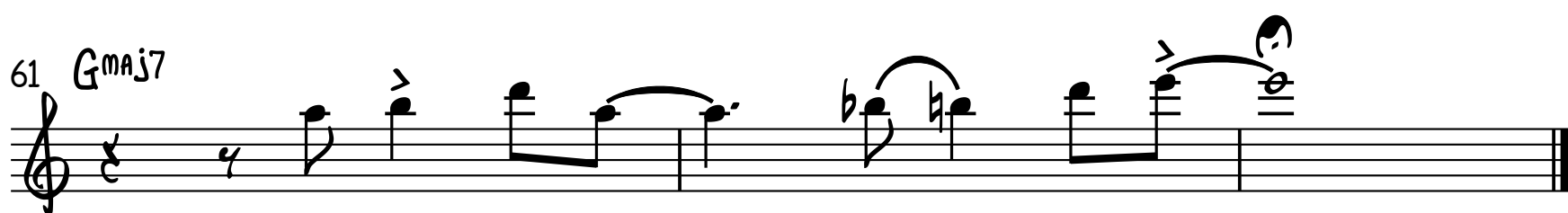


TENOR SAXOPHONE
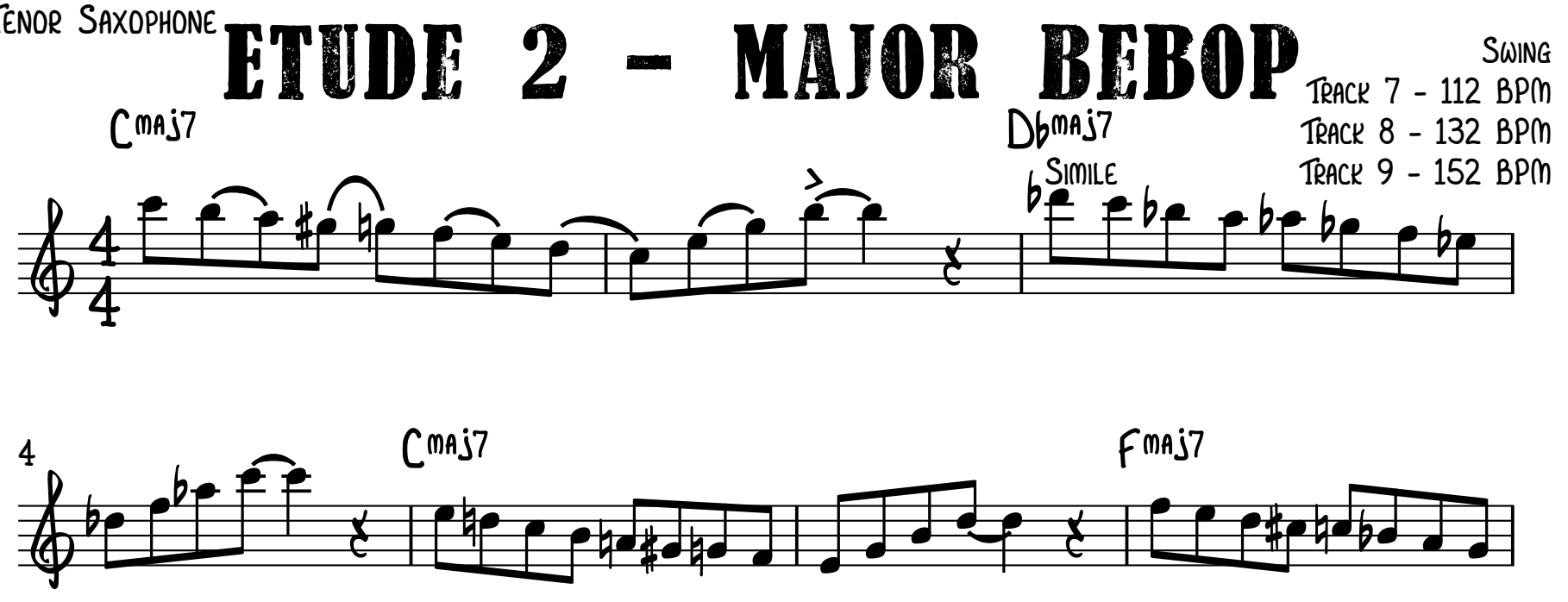

${ }^{8} \mathrm{Em}^{7}{ }^{A 7}{ }^{\mathrm{Dmij}}$

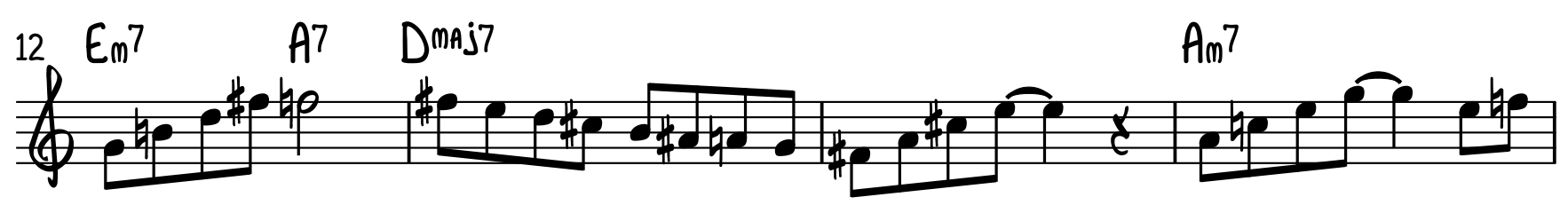

Gimmal)

${ }^{20}={ }^{27^{7}}$

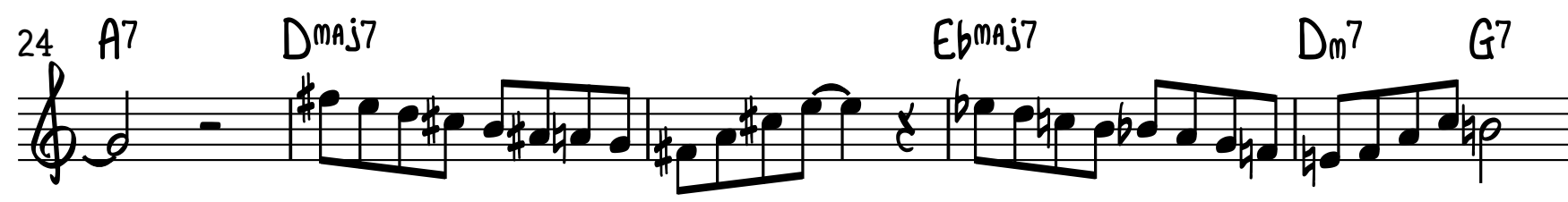

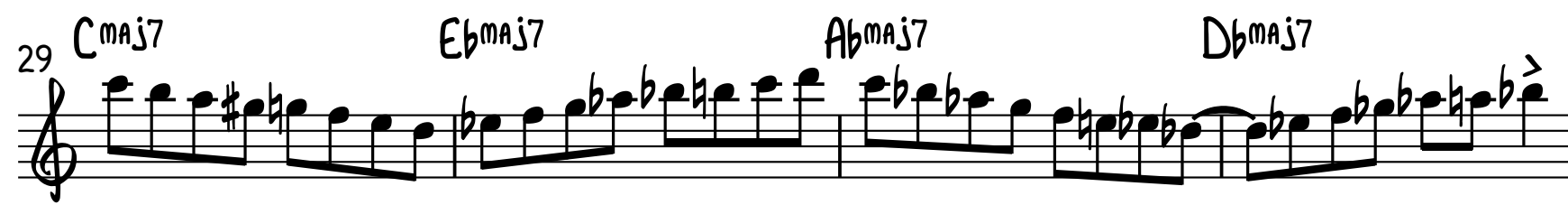


233

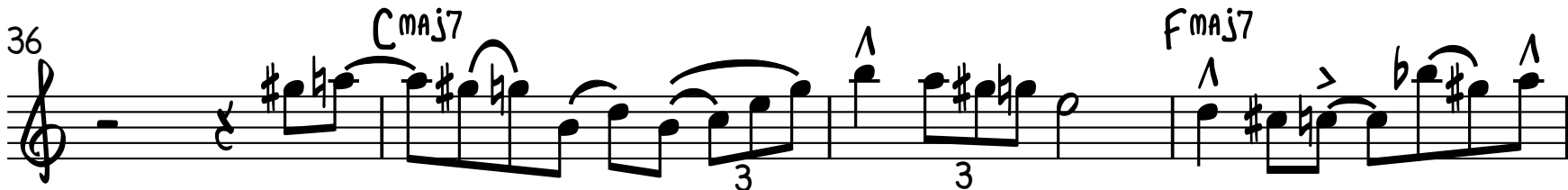

$E_{m} 7 \quad A 7 \quad$ Dmaj7

Ebmaj7

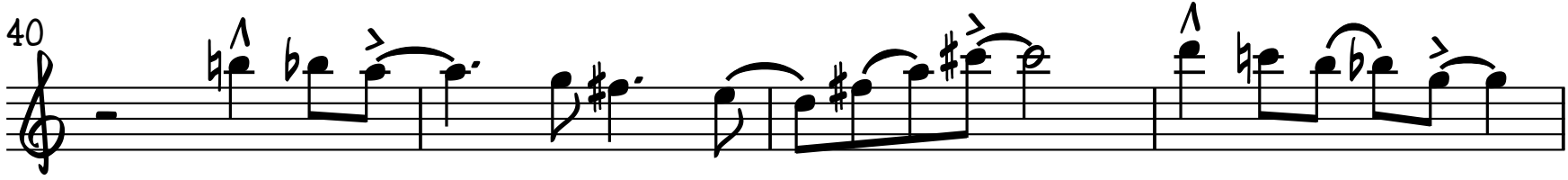

$\underbrace{E 7}$

$\mathrm{A}^{47} \mathrm{Am}^{7}$

$\begin{array}{lll}G_{m}(m A j 7) & F \#_{m} & B 7\end{array}$

\#攺

(55 $E_{m}^{7}$

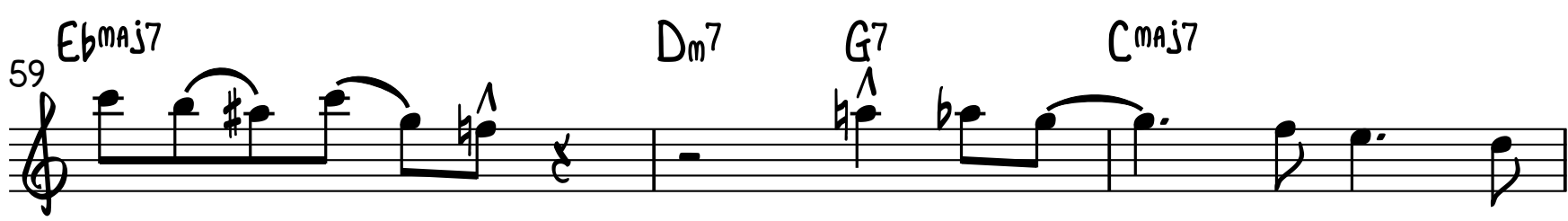

62 Ebmaj7 Abmaj7 Dbmaj7 Cmaj7

$\#$ (4) 
TENOR SAXOPHONE

\section{ETUDE 3 - DOMINANT BEBOP}

TRACK 13 - 132 BPM

TRACK 14 - 152 BPM

TRACK 15 - 172 BPM

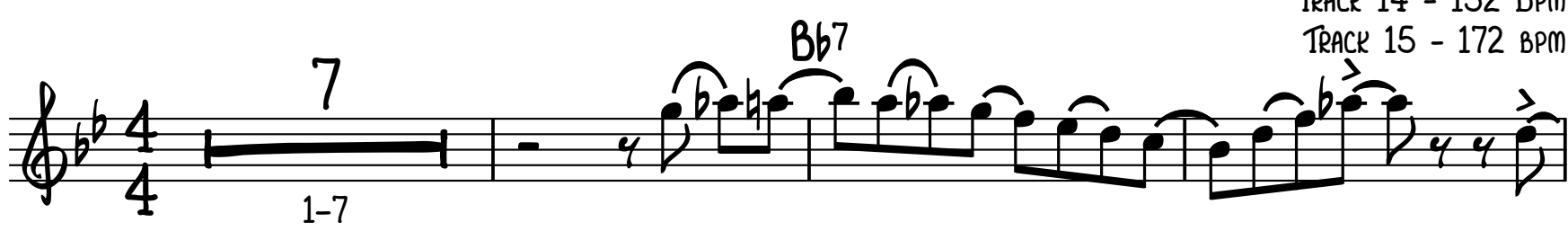

$f^{b-2}$
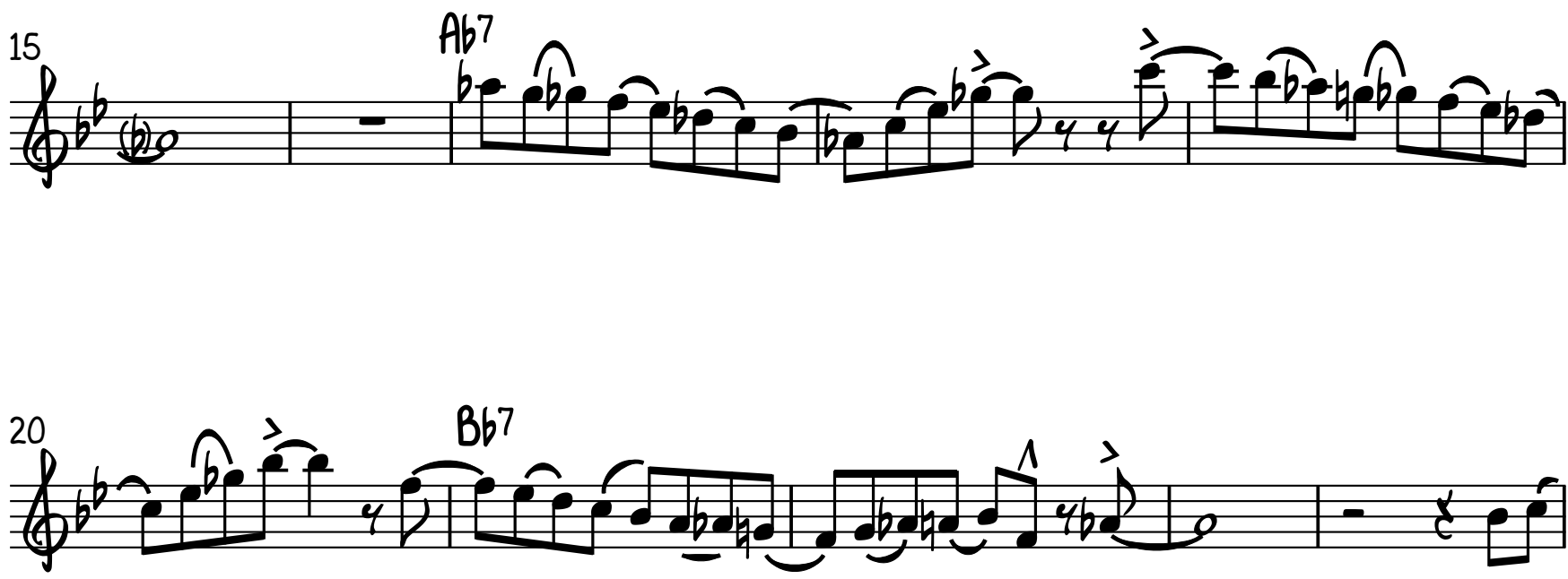

$25 b^{37}$

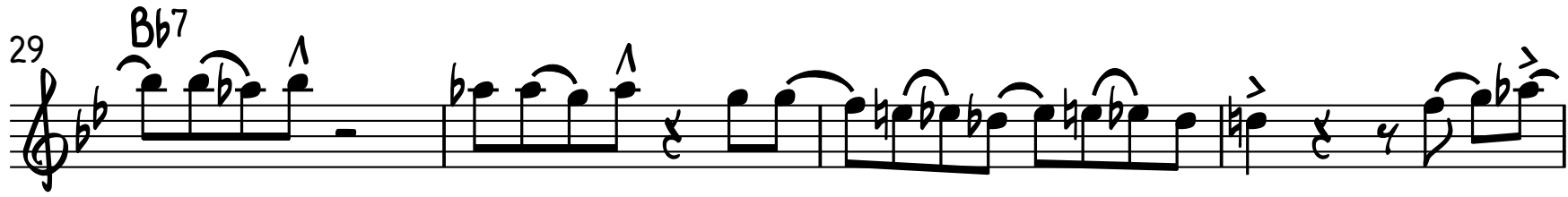


2

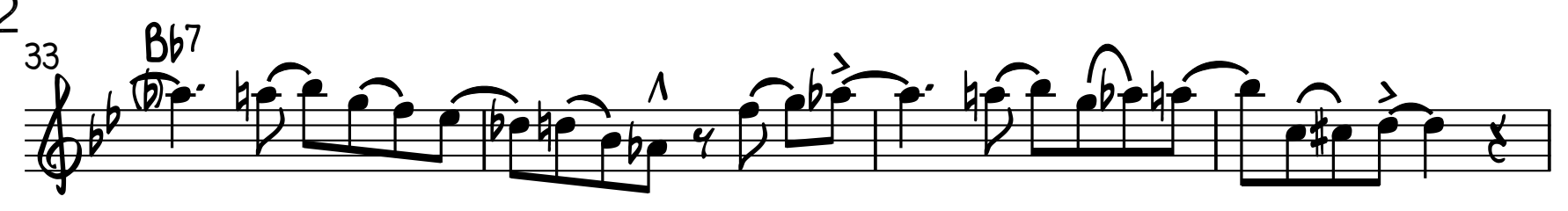

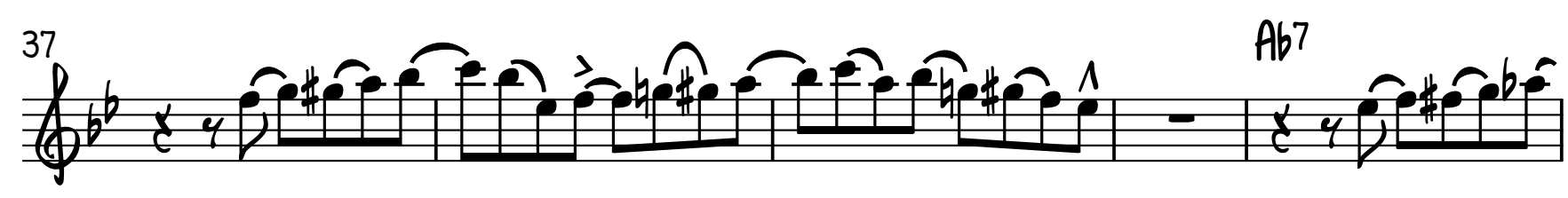

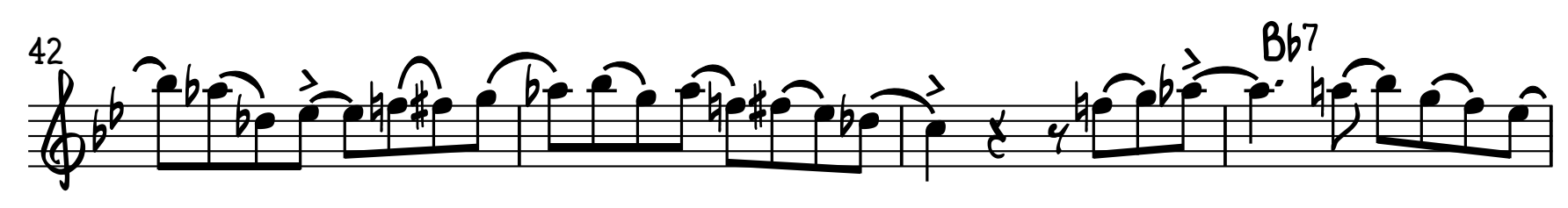
18. (50 ${ }^{67}$

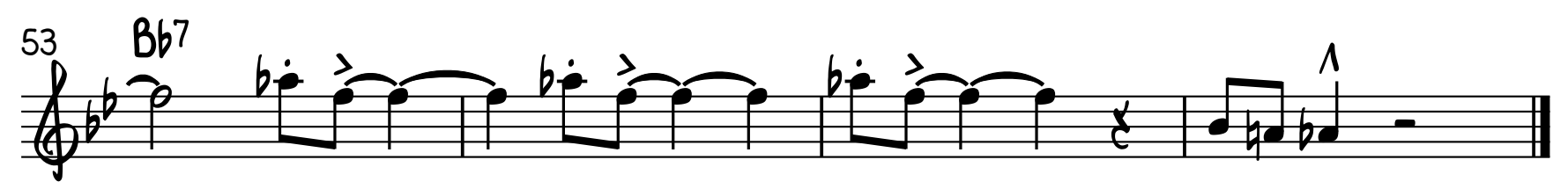


TENOR SAXOPHONE

\section{ETUDE 4 - DOMINANT BEBOP}

D7

$E 67$

(4) ${ }^{D^{7}}$

TRACK 16 - 112 BPM (4) 4 12:

${ }^{5}{ }^{G 7}$ (4)

$\sum_{10}^{E b 7}$

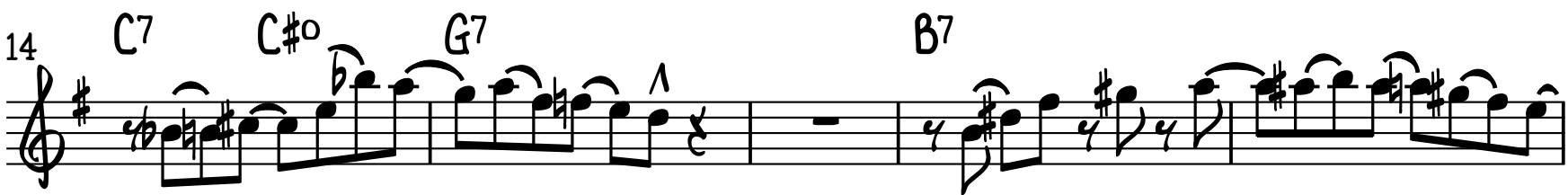

${ }^{19}{ }^{E 7}$ (1)

$23{ }^{D^{7}}$

$2^{28}$ 
TENOR SAXOPHONE

2

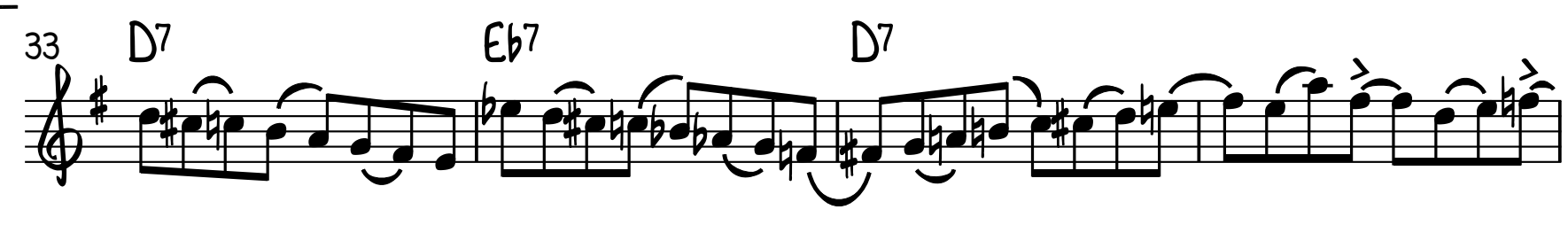

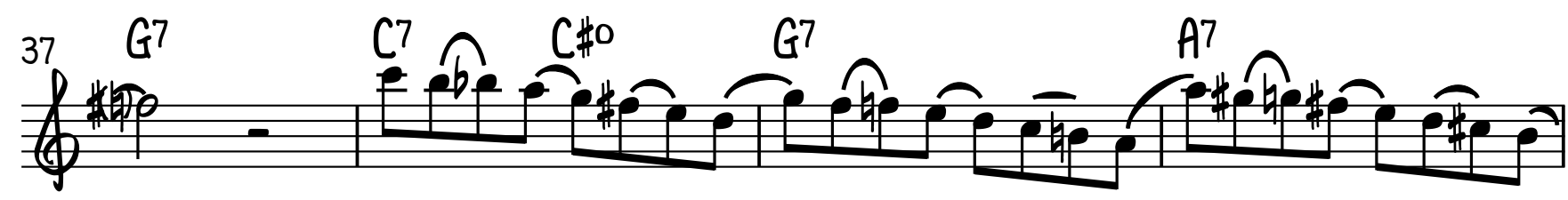

(1)

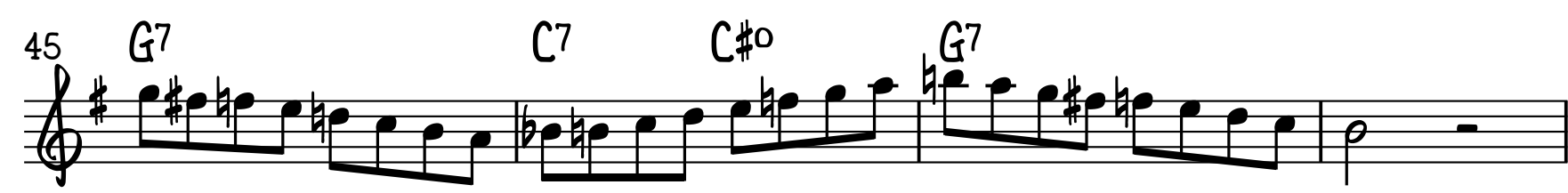

497

53 ${ }^{A 7}$

(57) $D^{7}+a^{67}$

G7

C7 C\#O

G7

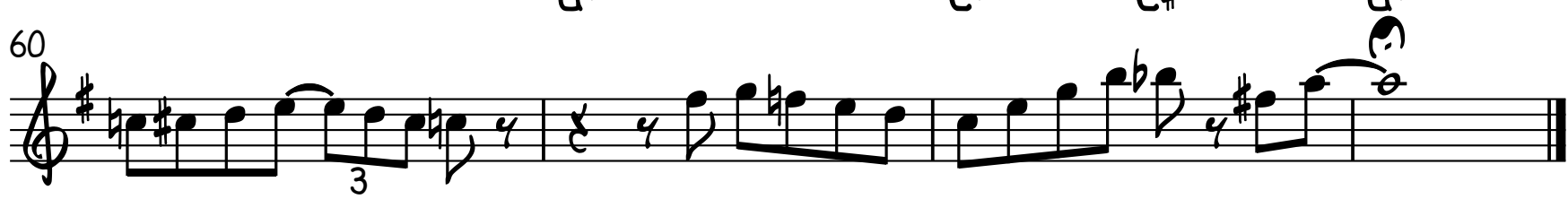


TENOR SAXOPHONE

ETUDE 5 - MINOR BEBOP

TRACK $31-100$ BPM TRACK 32 - 120 BPM

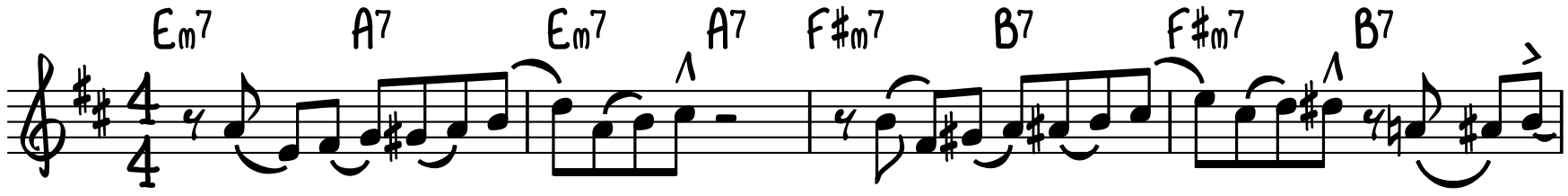

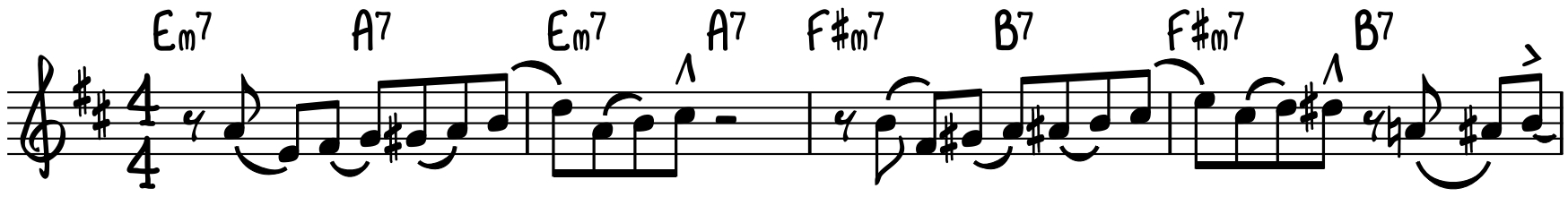

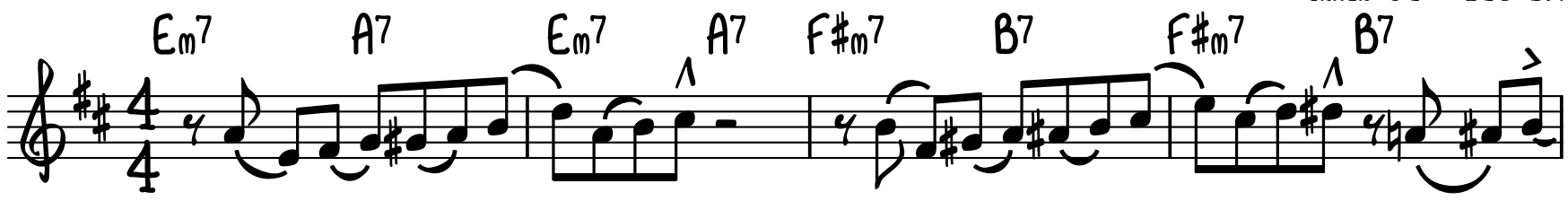

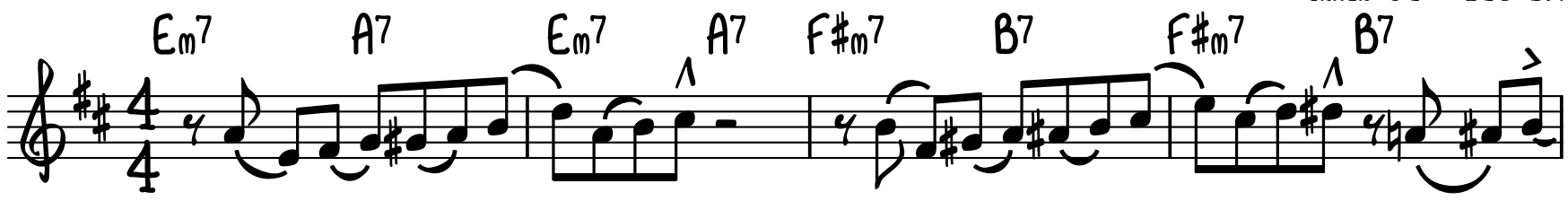

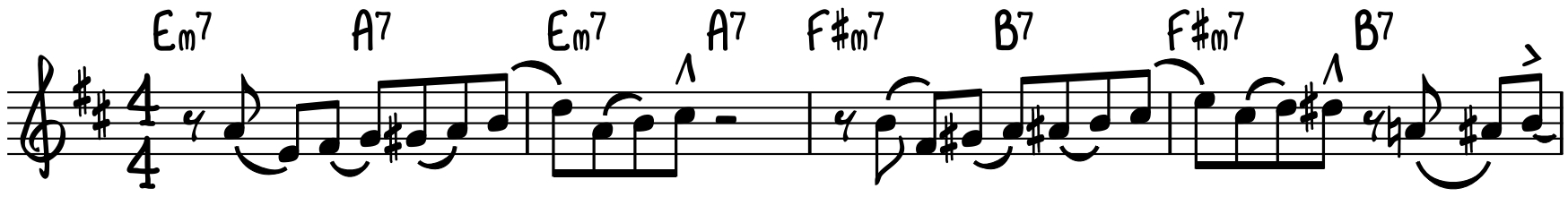

TRACK 34 - 140 BPm

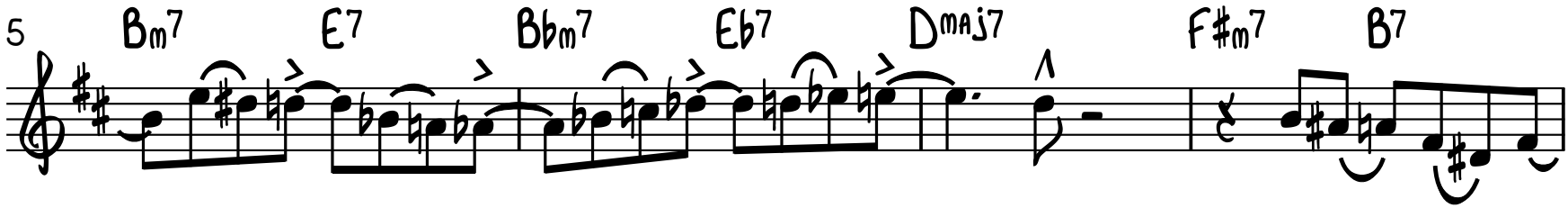

$9 \begin{array}{llllllll}E_{m} 7 & A 7 & E_{m} & A 7 & F^{*} 7 & B 7 & F \#_{m} 7 & B 7\end{array}$

(4)

$13{ }^{\mathrm{Bm} 7}$

$\begin{array}{llllll}17 & A_{m} 7 & D^{7} & A_{m} 7 & D^{7} & G^{m a j 7}\end{array}$

(4)

$21 \quad B_{m} 7 \quad E^{7} \quad B_{m} 7 \quad E^{7} \quad A^{7}$

(4)

$\begin{array}{lllllllll}25 & E_{m} 7 & A 7 & E_{m} & A 7 & F \sharp_{m} 7 & B 7 & F \#_{m} & B 7\end{array}$

4

$29 \begin{array}{lllllll}\mathrm{Bm}^{7} & E 7 & \mathrm{Bbm}^{7} & E b 7 & D^{m a j 7} & \mathrm{~F}_{m} 7 & B 7\end{array}$

(4)

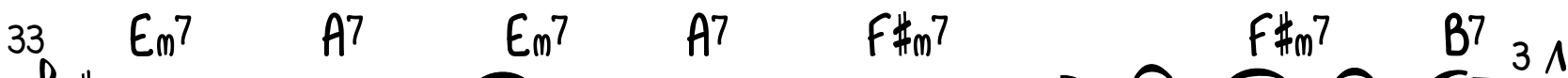
(4) $\begin{array}{lllllll}B_{m} 7 & E 7 & B b_{m} 7 & E b 7 & D^{m A j 7} & F \sharp_{m} 7 & B 7\end{array}$

(4) 
2

TENOR SAXOPHONE

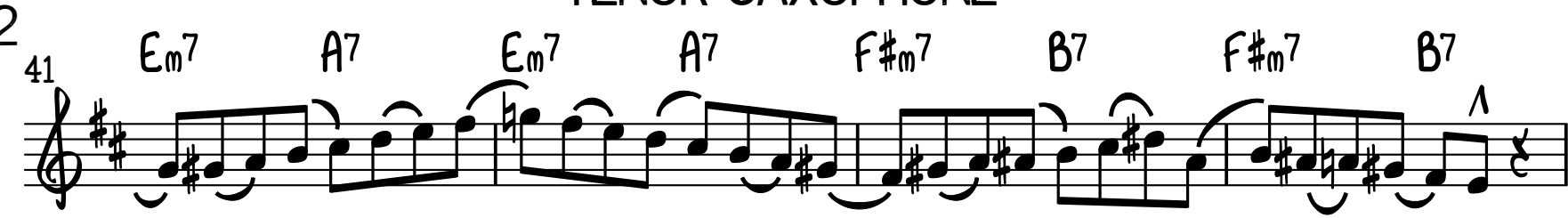
$\begin{array}{llllll}45 & B_{m}{ }^{7} & E 7 & B b_{m}{ }^{7} & E 67 & D^{m a j 7}\end{array}$

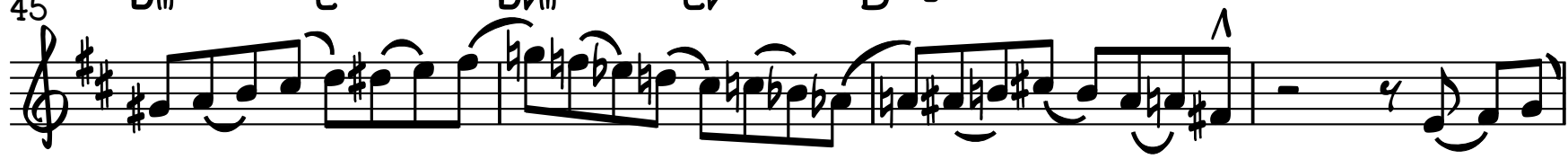
$\begin{array}{llllll}49 & A_{m}{ }^{7} & D^{7} & A_{m}{ }^{7} & D^{7} & G^{\text {maj7 }}\end{array}$ 6) $\begin{array}{llllll}53 & B_{m}{ }^{7} & E 7 & B_{m}{ }^{7} & E 7 & A^{7}\end{array}$

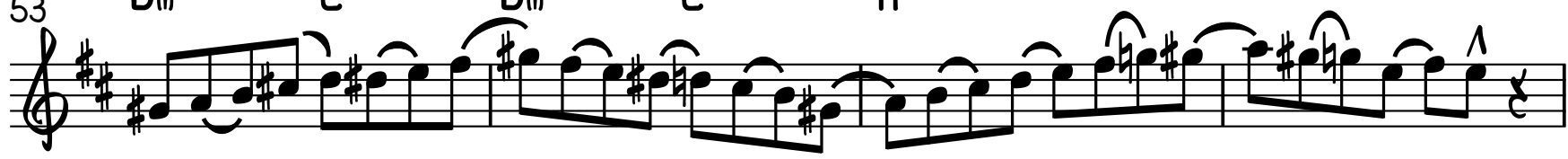
${ }_{B m^{7}}^{E^{7}} A_{E 7}^{A 7} E_{B b m^{7}}^{E^{7}}{ }_{E b 7}^{A 7}$

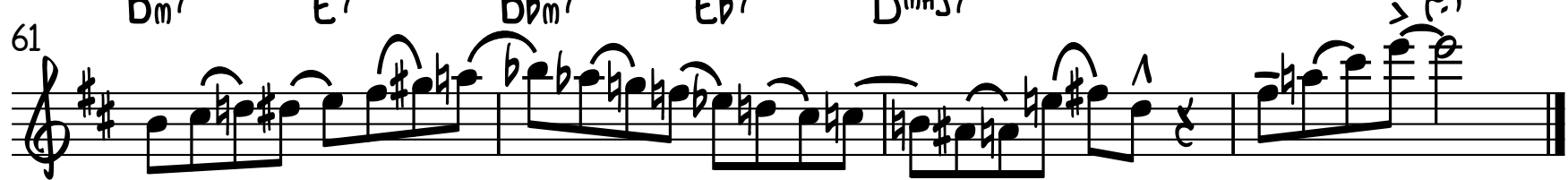


Exercises in Developing Bebop Scale Language

for Collegiate Jazz Students

\author{
Lars Swanson \\ West Virginia University
}

\author{
Trombone Edition
}


TROMBONE

EVEN 8THS

\section{ETUDE 1 - MAIOR BFBOP Gmaj7} $2 \frac{4}{4}$

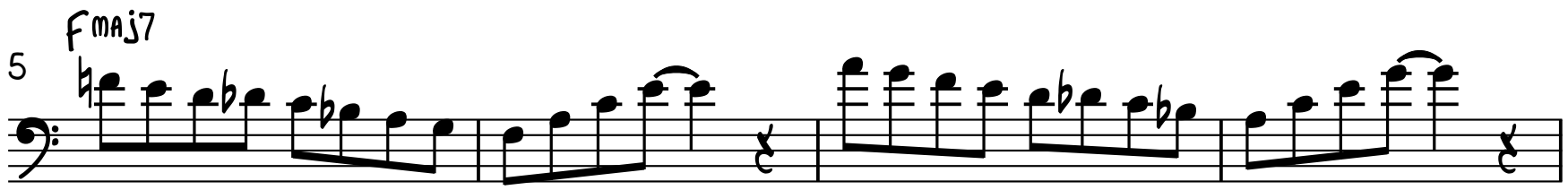

Ebmaj7

13 Fmaj7

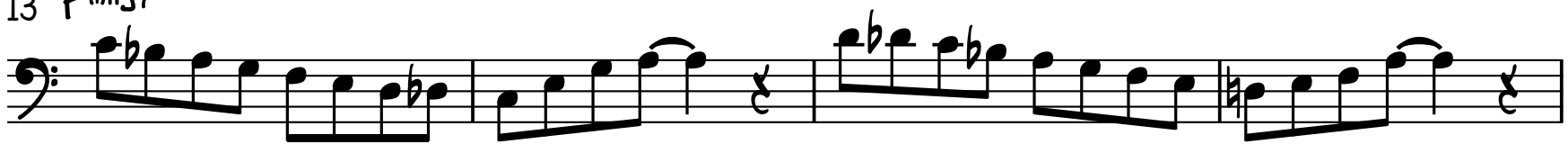

17 Gmaj7 Cmaj7

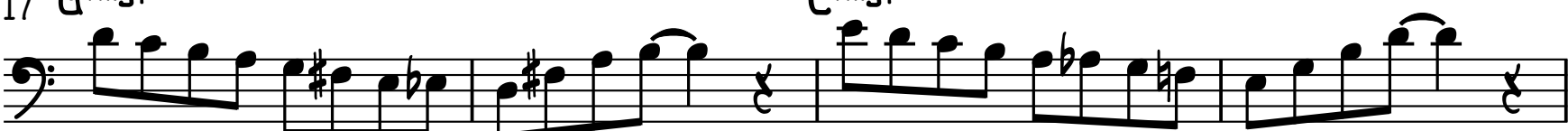

21 FmAj7 Bbmaj7

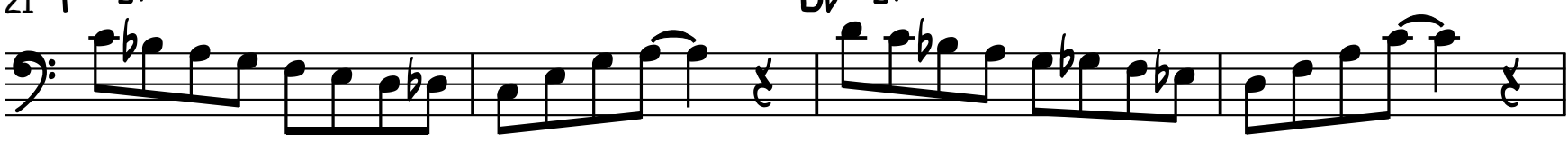

25 Ebmaj7

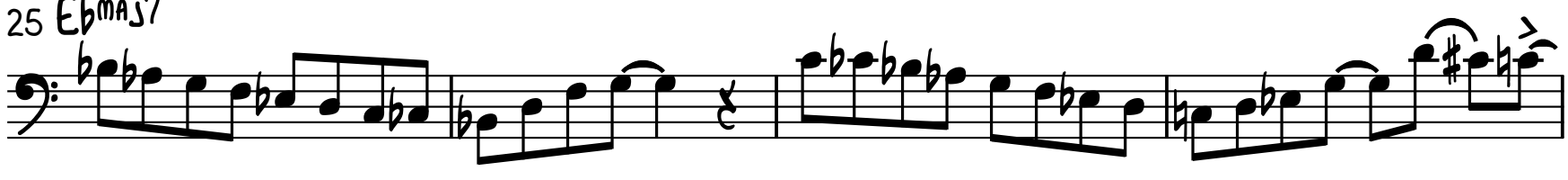

29 Fmaj7

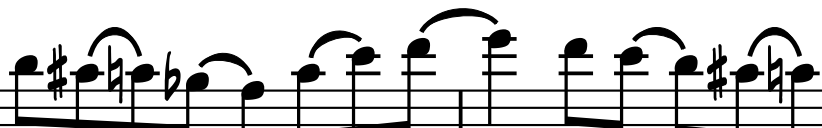

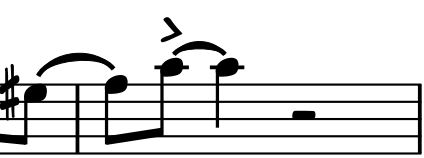


2 G3mi7 36.

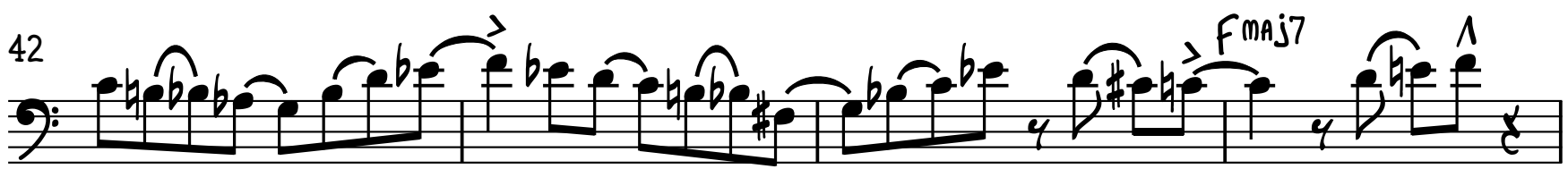

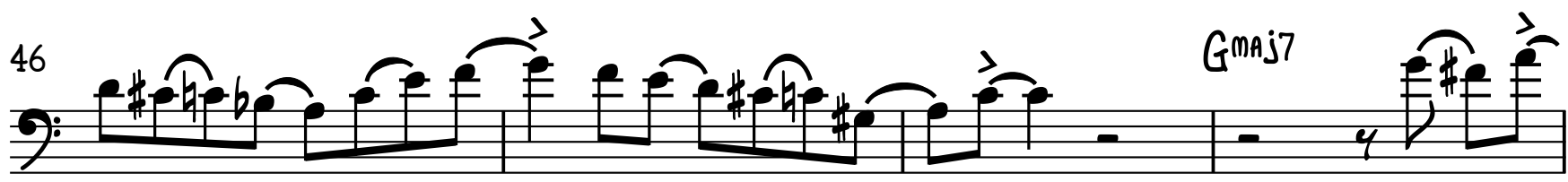

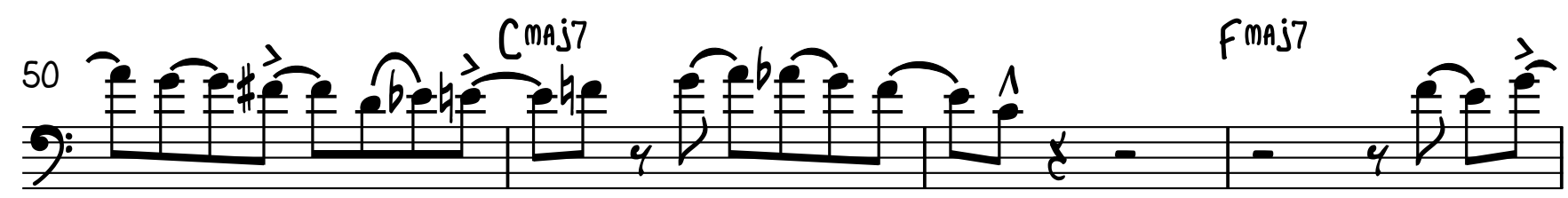
54

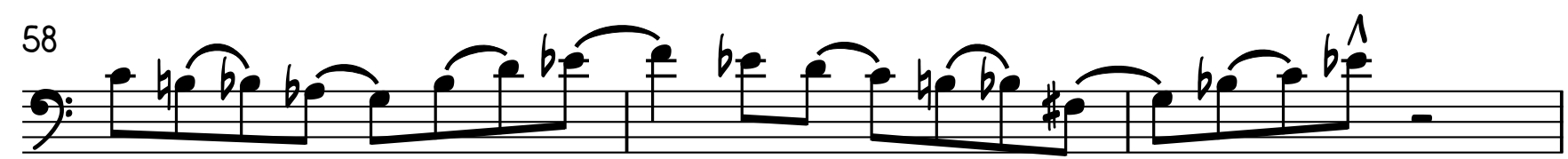

61 Fmaj7

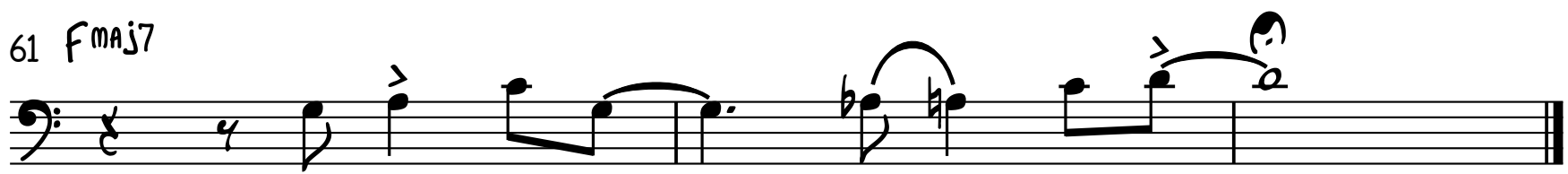


Bbmaj7

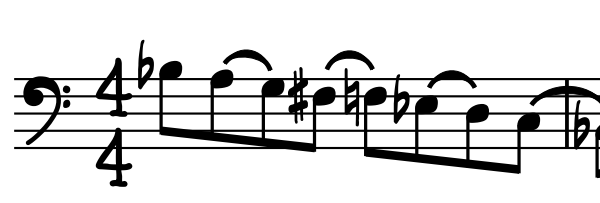

BmAj7

SIMILE

TRACK 7 - 112 BPM

TRACK 8 - 132 BPM

TRACK 9 - 152 BPM
Bbmaj7
Ebmaj7
$D_{m} 7$
G7

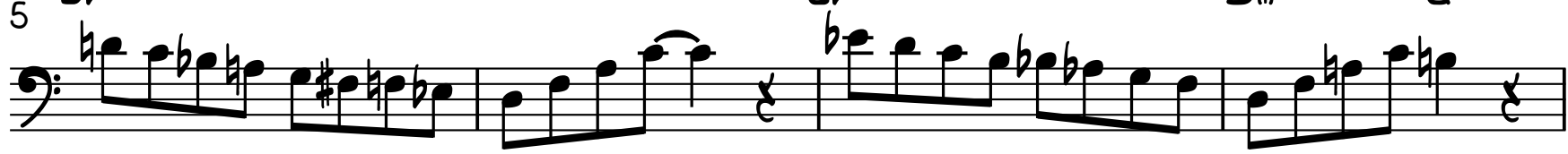

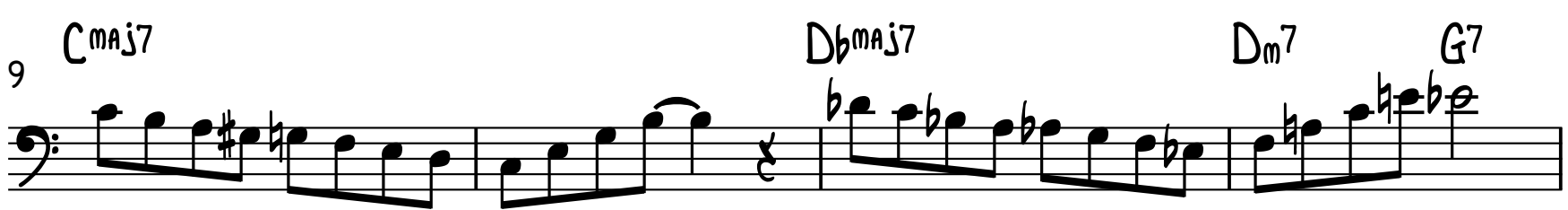

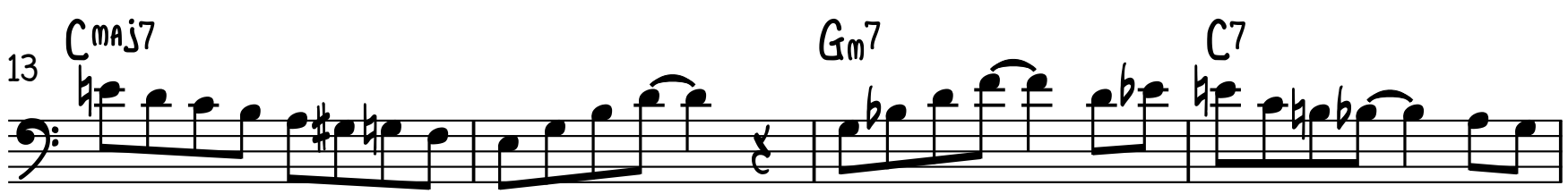

17 F'MAj7

$21 \mathrm{Em}^{7}$

${ }_{21}^{E_{m}{ }^{2}}$

$21 \mathrm{Em}^{7}$

${ }_{21}^{E_{m}{ }^{2}}$

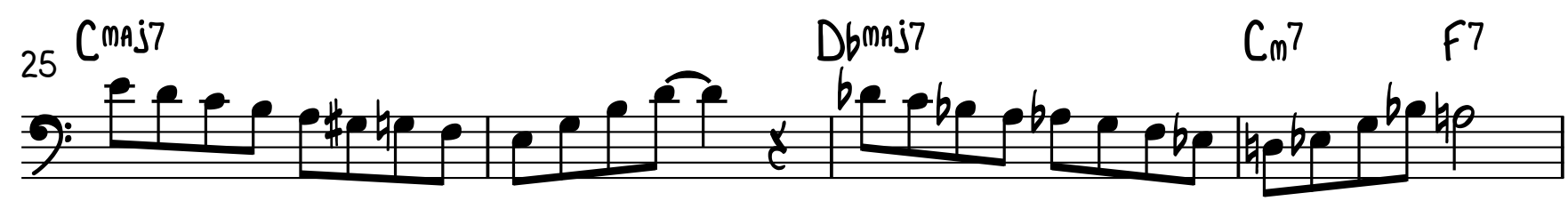

29 Bbmaj7 Dbmaj7 $\quad$ Gbmaj7 $\quad$ Bmaj7

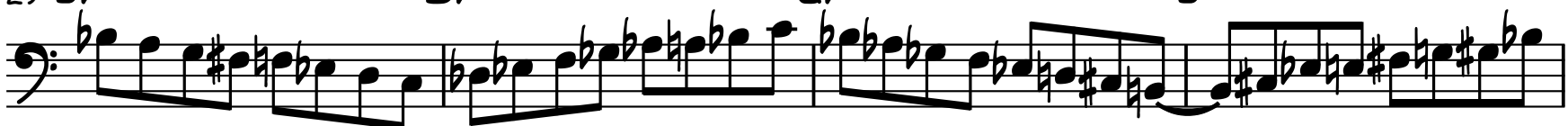


TROMBONE

$2{ }_{33}^{\text {Bmmaj7 }} \rightarrow$ Bmaj7 2.8

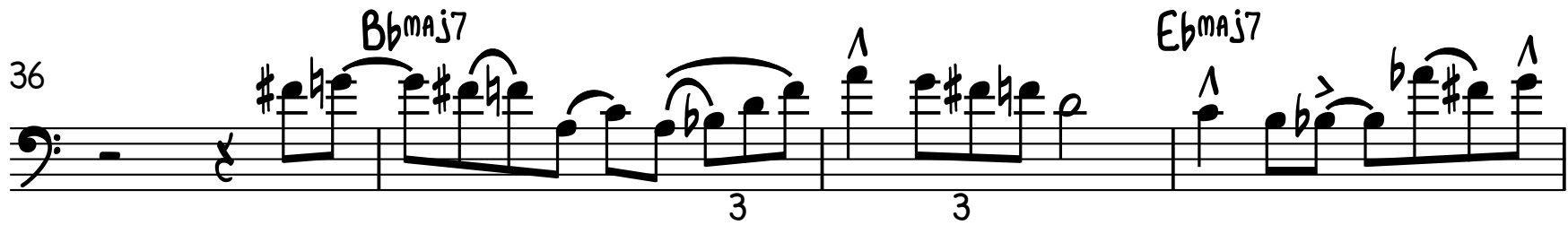
Dm 7 G7 $\quad$ Cmaj7
Dbmaj7

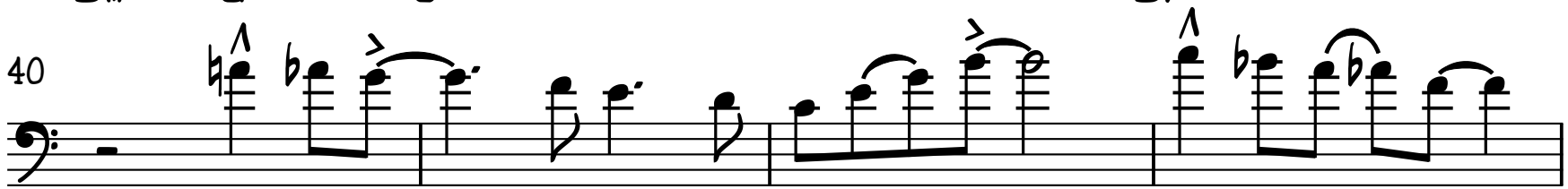

$\begin{array}{lllll}D_{m} 7 & G 7 & C^{\text {maj7 }} & G_{m}^{7} & C 7\end{array}$

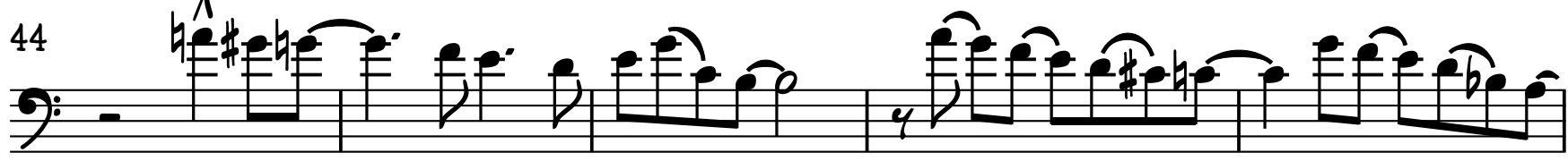

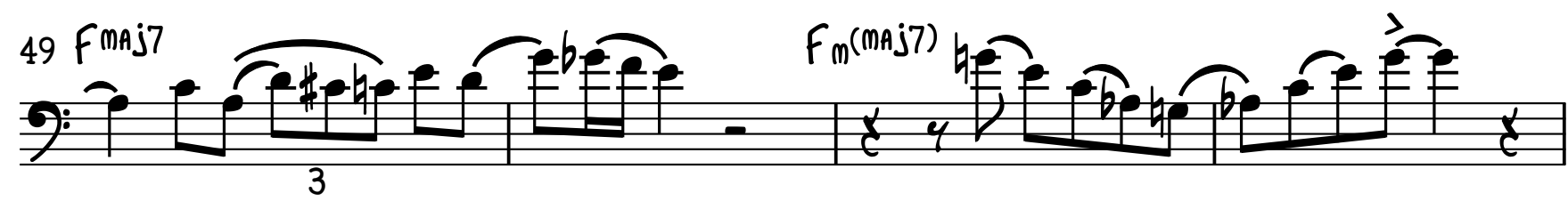

93

Cini7

91 


\section{ETUDE 3 - DOMINANT BEBOP}

TROMBONE

BOOGALOO:

TRACK 13 - 132 BPM

TRACK $14-152$ BPm

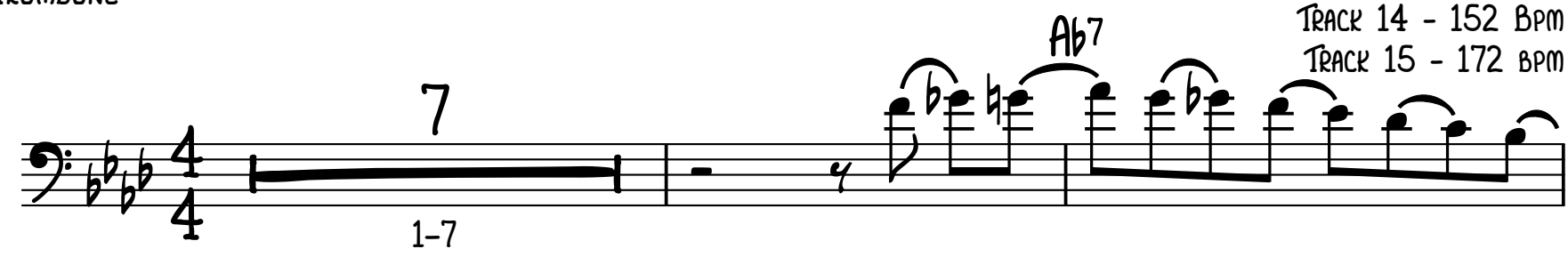

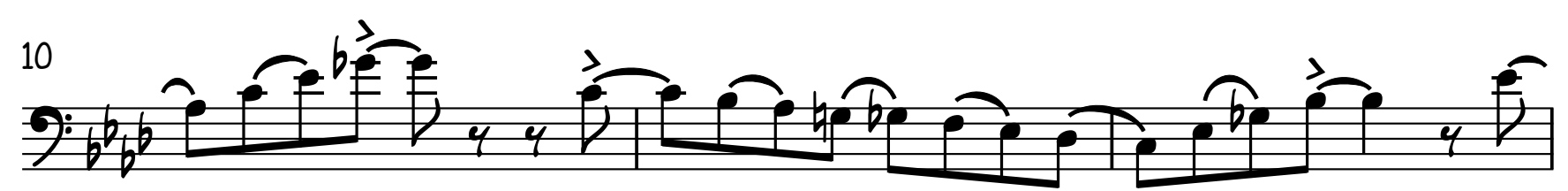

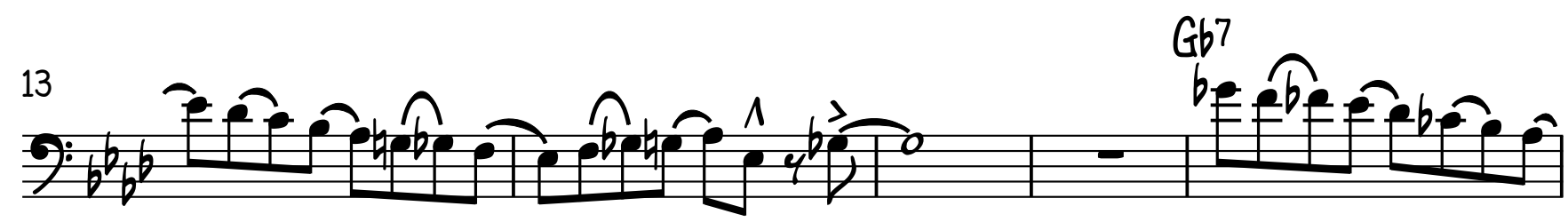

\%:bife

$22: b_{b} b^{2}$

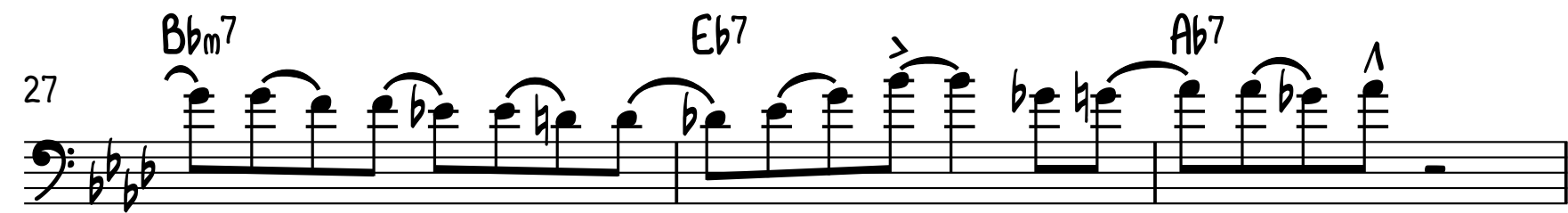

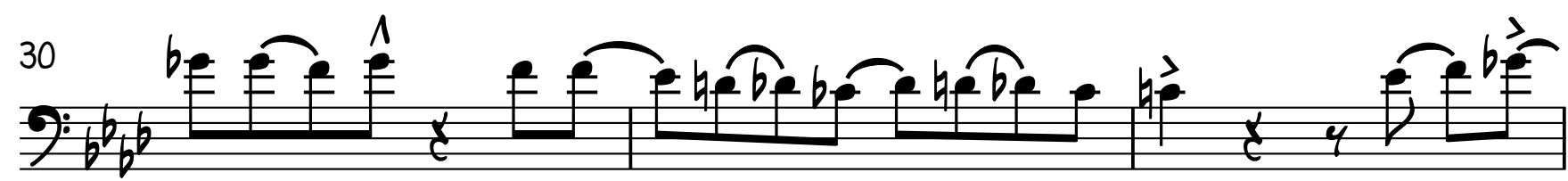


$2 \quad \mathrm{Ab7} \quad$ TROMBONE

200

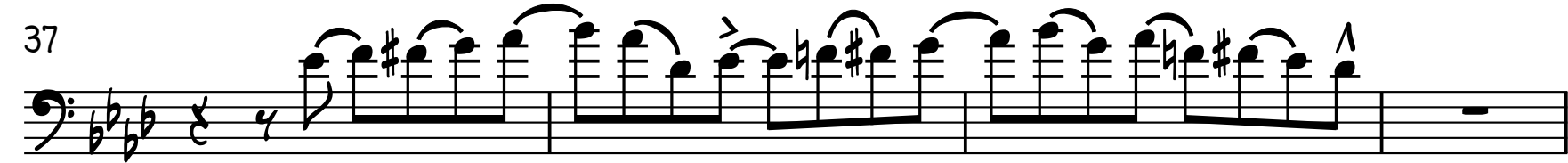

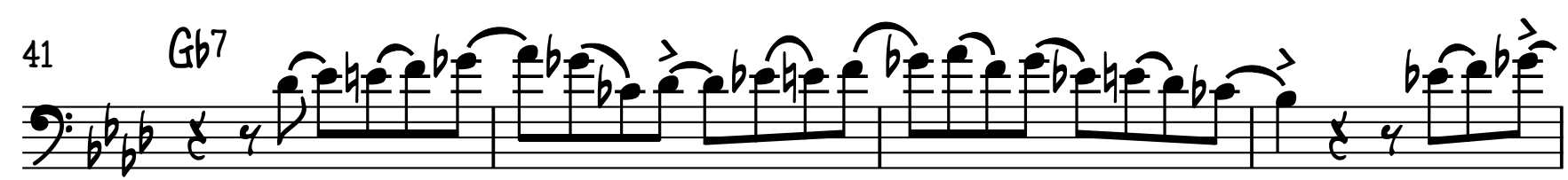

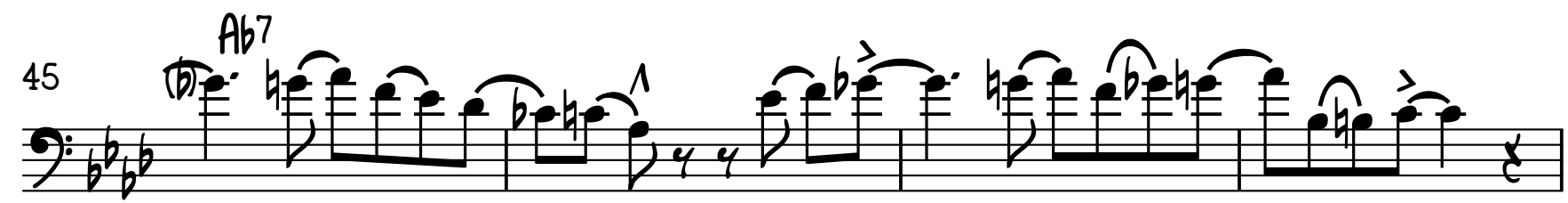

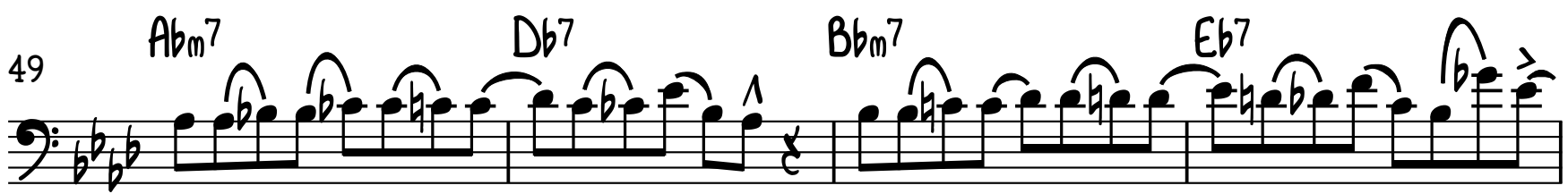

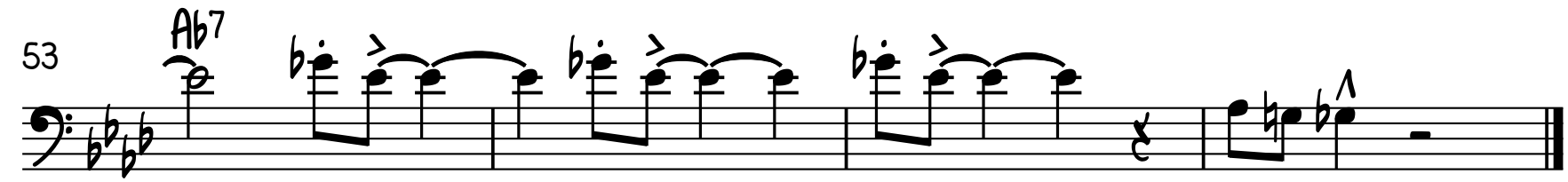


2

TROMBONE

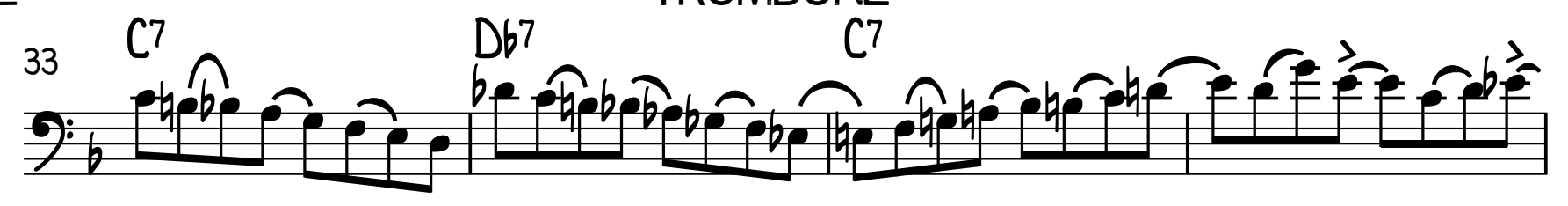

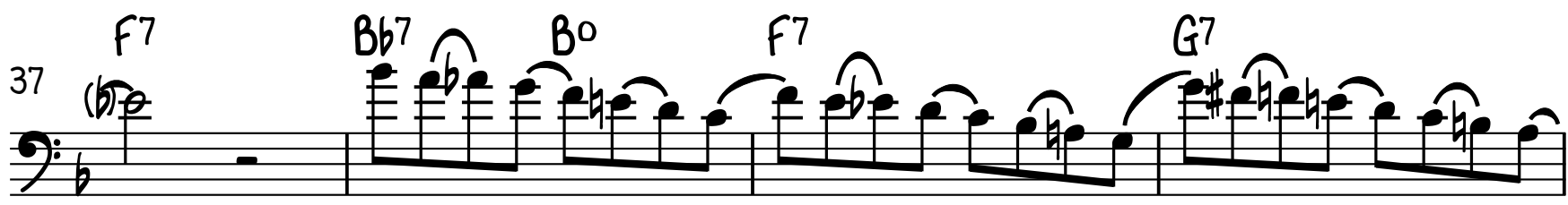

$\begin{array}{lll}C 7 & D 67 & C 7\end{array}$

$2 \frac{1}{1}$

${ }_{25}^{47}$

20

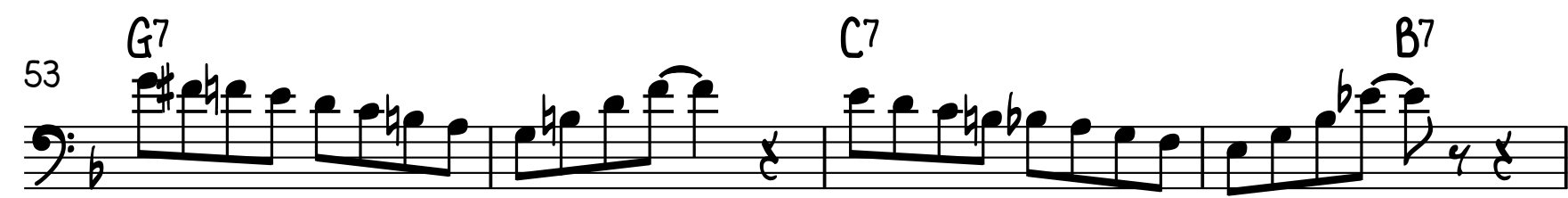

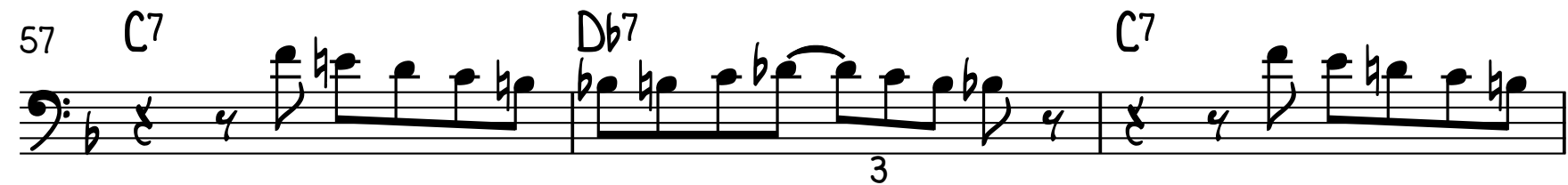

F7

Bb7 Bo

F7

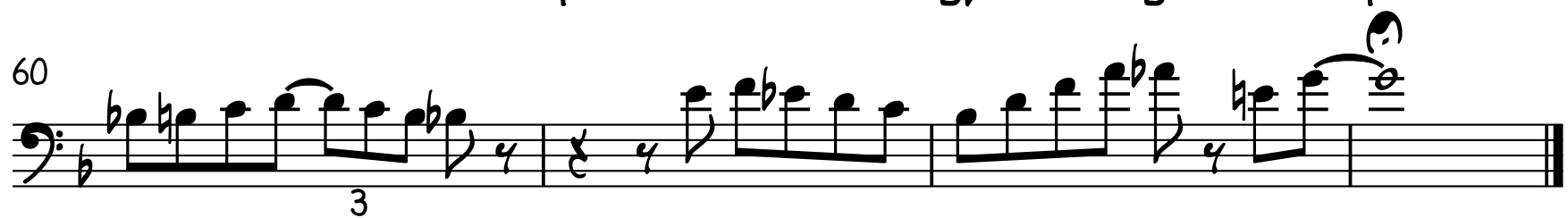




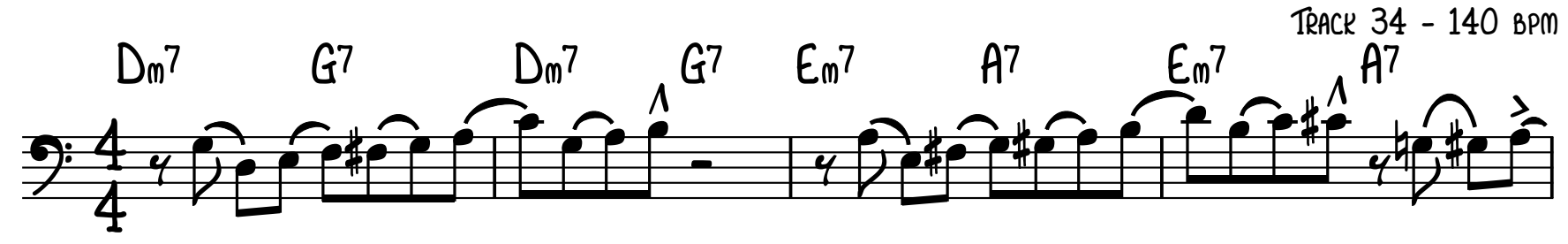
$5 \mathrm{Am}^{7}$ $9 \mathrm{Dm}^{\mathrm{G}} \mathrm{Dr}^{7}$ $13 \mathrm{Am}^{7}$ $17 \mathrm{Gm}^{7}$ $21 \mathrm{Am}^{7}$ $25 D_{m} 7 \quad G 7 \quad D_{m}^{7} \quad G^{7} \quad E_{m}^{7} \quad A^{7} \quad E_{m}^{7} \quad A^{7}$

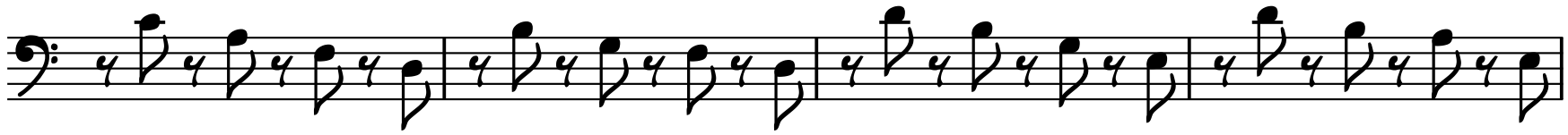

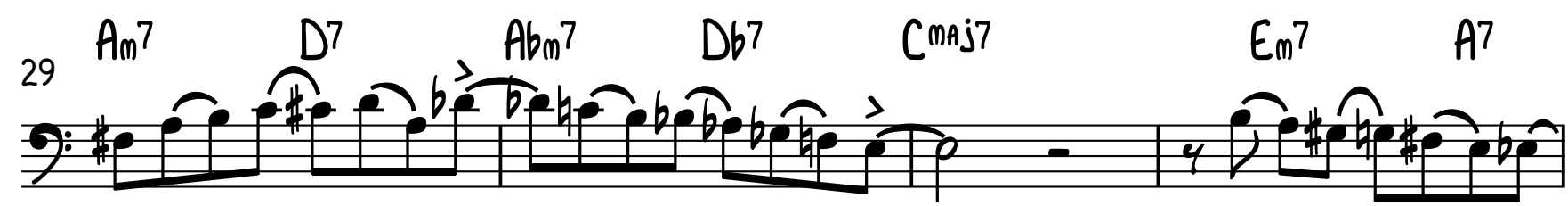


TROMBONE

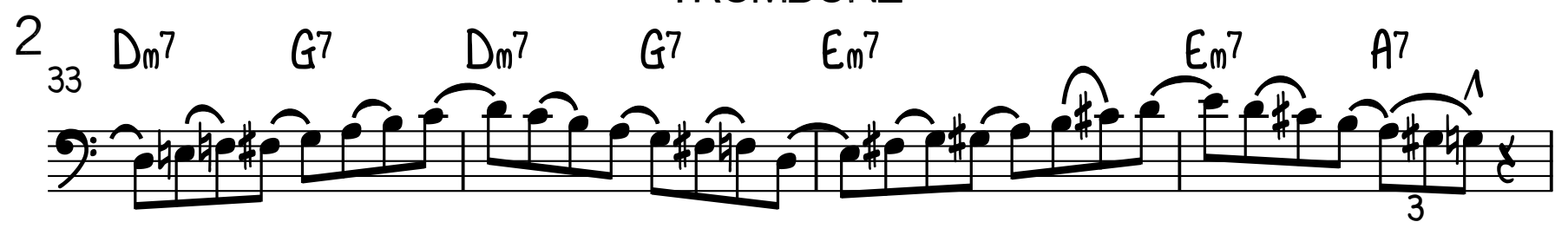

$\begin{array}{llllllll}A m & D 7 & A b m & D b 7 & C & E^{m a j 7} & A 7\end{array}$

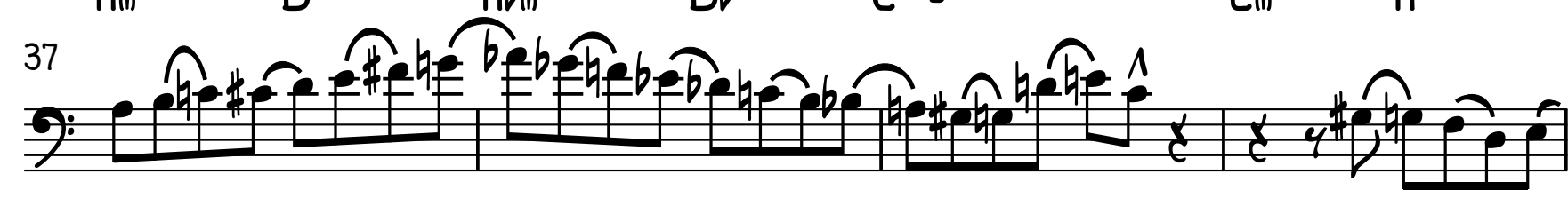

$\begin{array}{llllllll}D_{m} 7 & G 7 & D_{m} 7 & G 7 & E_{m}^{7} & A 7 & E_{m}^{7} & A 7\end{array}$

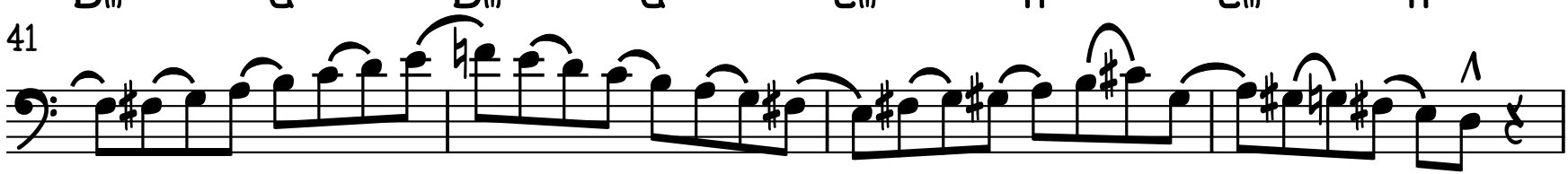

$\begin{array}{lllll}A m & D 7 & A b m & D b 7 & \text { Cmaj7 }\end{array}$

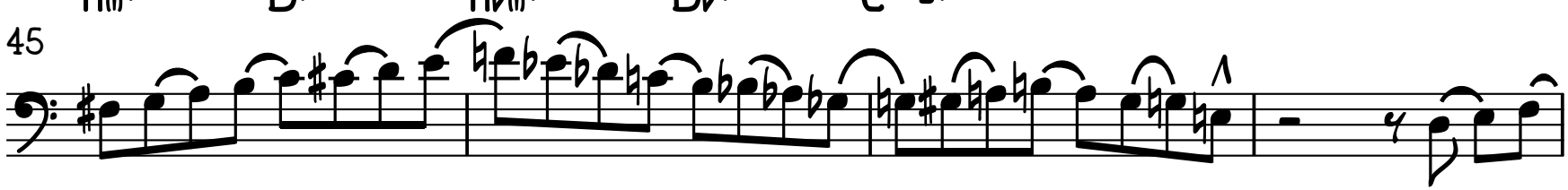

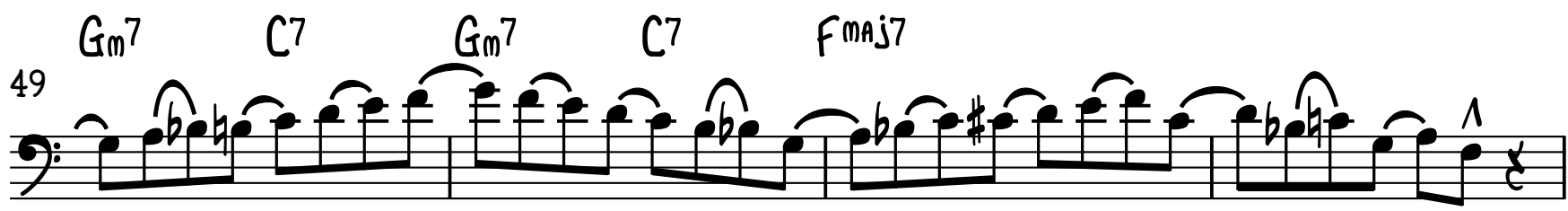

$\begin{array}{lllll}A_{m} 7 & D^{7} & A_{m} 7 & D^{7} & G^{7}\end{array}$

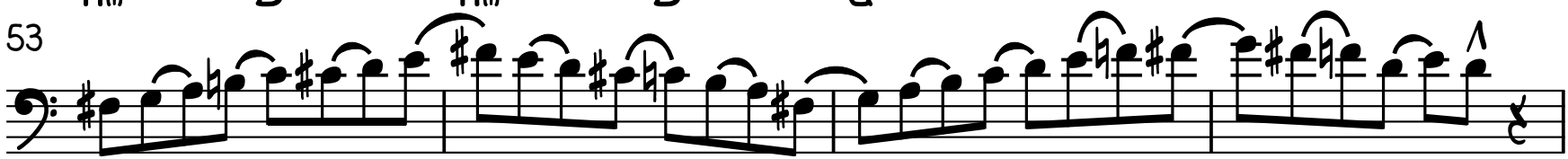

${ }_{57}^{\mathrm{Dm}^{7}} \mathrm{G}^{7}$

${ }^{61} \mathrm{Alo}^{7} \mathrm{D}$ 
Exercises in Developing Bebop Scale Language

for Collegiate Jazz Students

\author{
Lars Swanson \\ West Virginia University
}

\author{
Bass Edition
}




\section{SCALE EXERCISE 1 - MAJOR BEBOP SCALES}

SWING

TRACK 1 - 100 BPM

CmAj7

TRACK 2 - $120 \mathrm{BPM}$

TRACK 3 - 140 BPM

$\frac{4}{4}, \cdots$

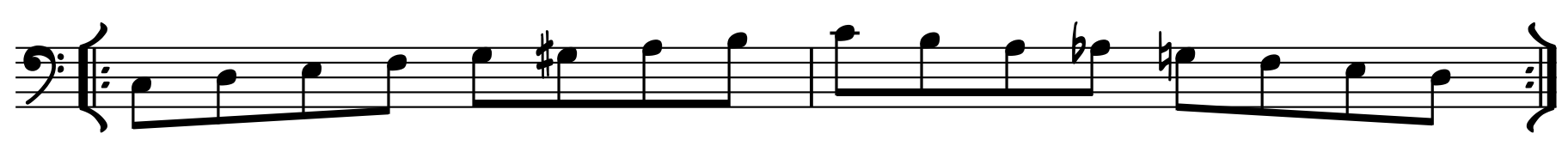
Fmaj7

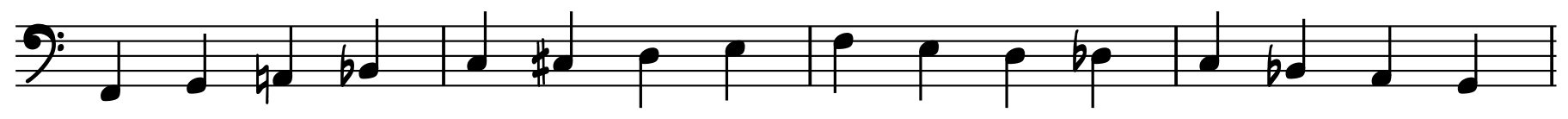

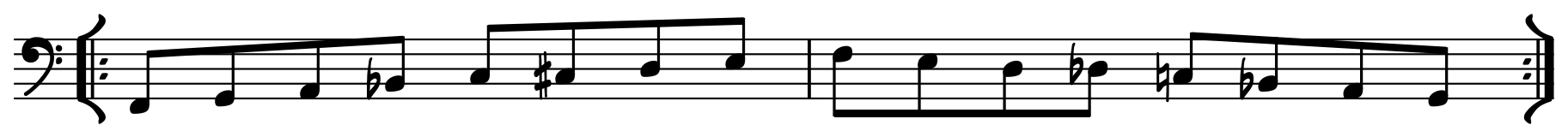

Bbmaj7

200 C C C C C

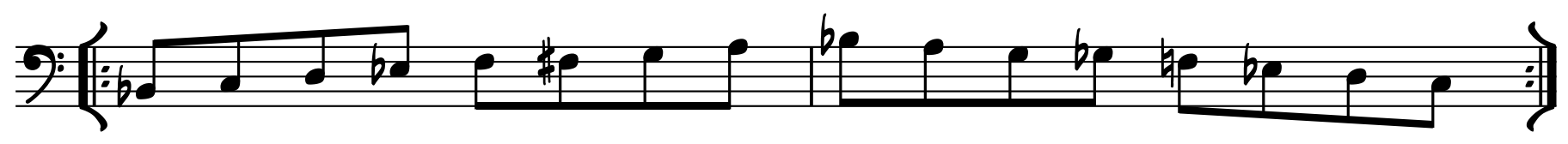

Ebmaj7

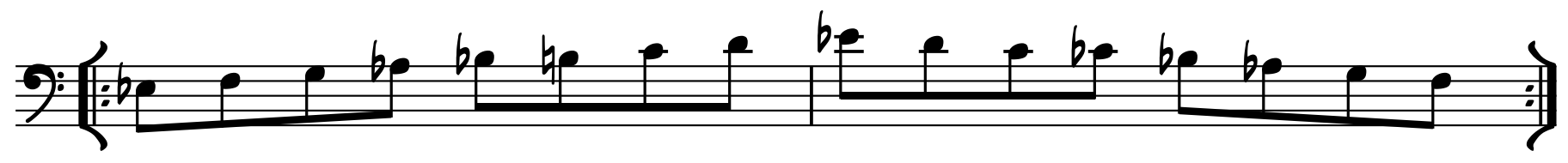
Abmaj7

2. C C C 


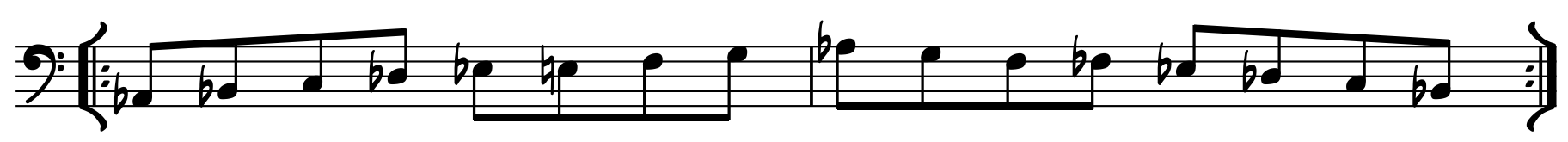

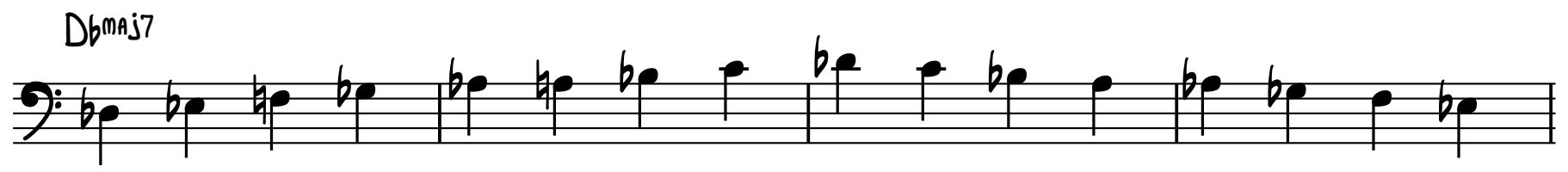

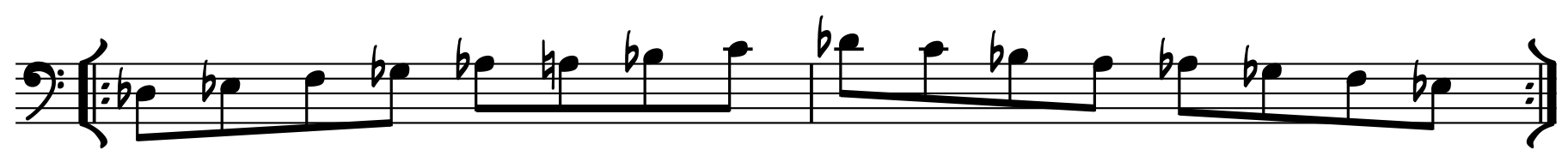
Gbmas7

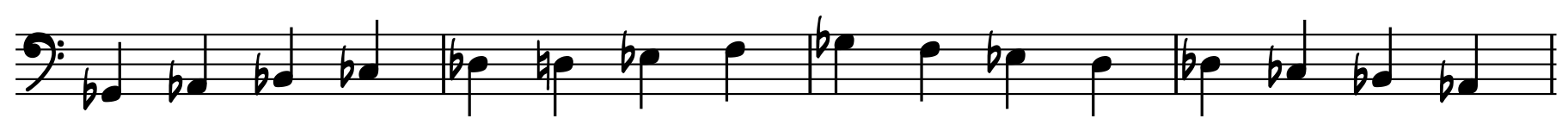

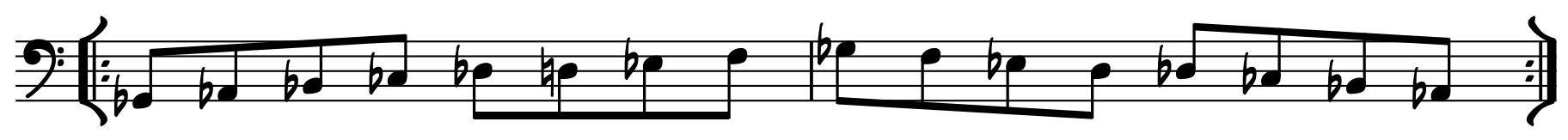
sow

7.

श्(; Emaj7

श.

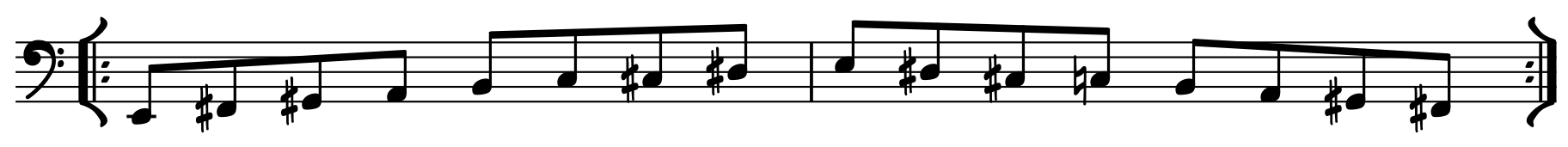
pars

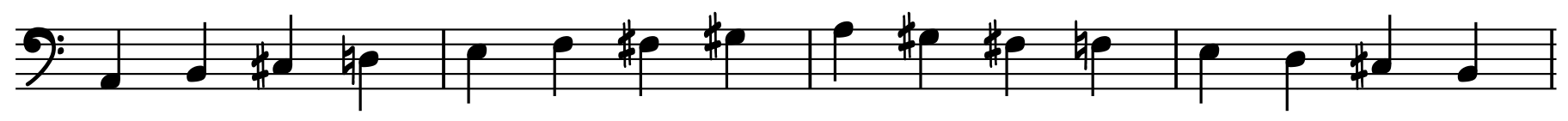



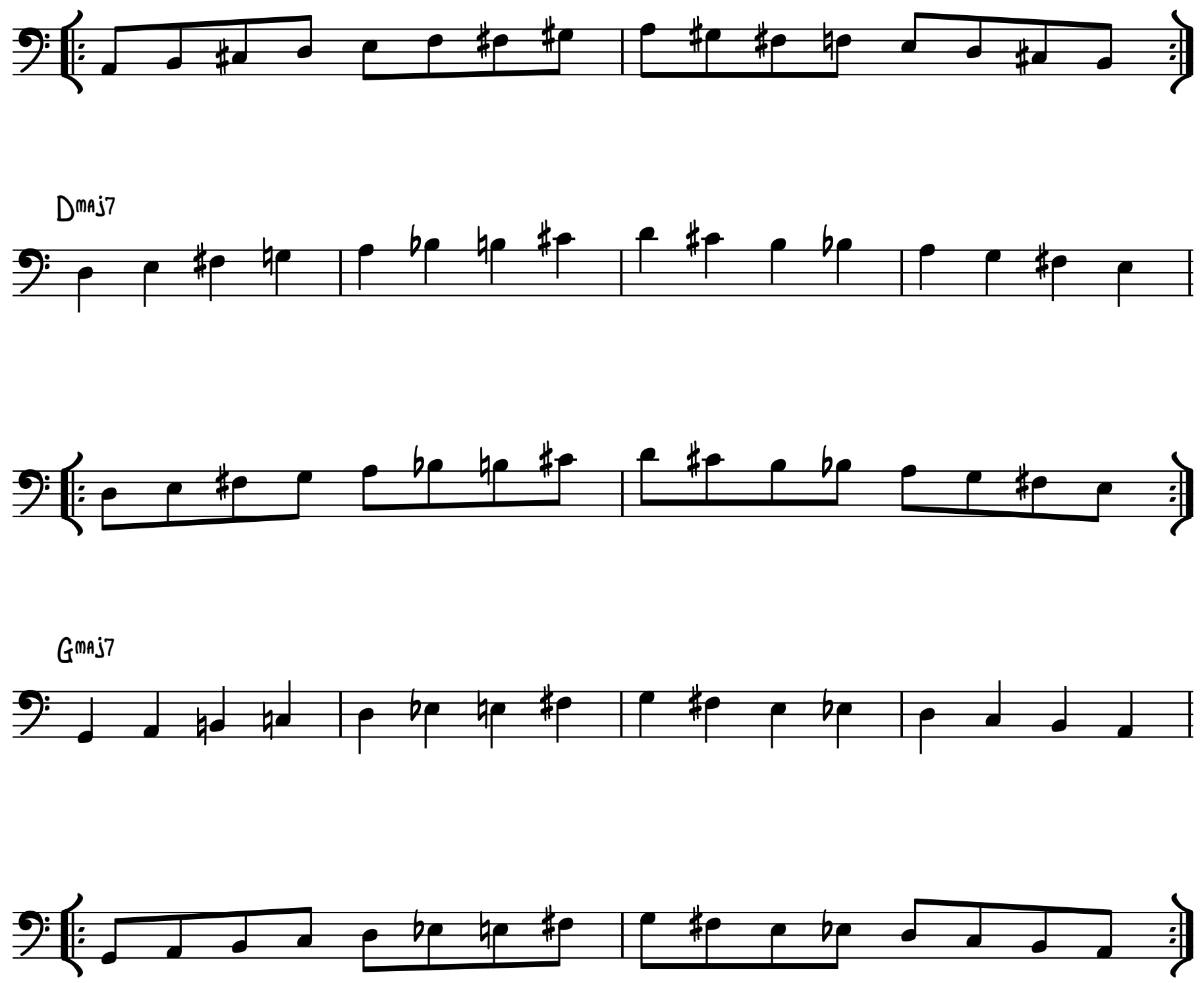

ONCE YOU HAVE MASTERED THIS AT THE FASTEST TEMPO, TRY TO

PRACTICE THESE SCALES STARTING ON THE 3RD, 5TH AND 6TH SCALE DEGREES.

CMAj7

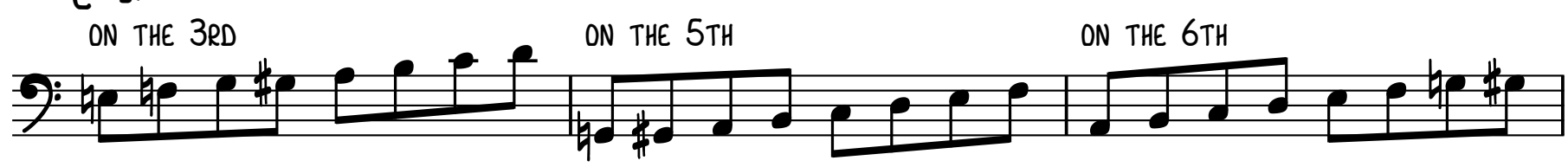




\section{SCALE EXERCISE 2 - DOMINANT BEBOP SCALES}

C7

- 5 :

安:

F7

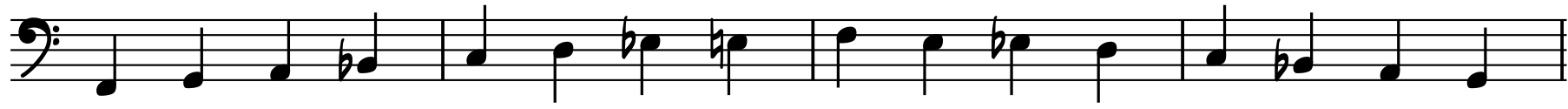

T:

Bb7

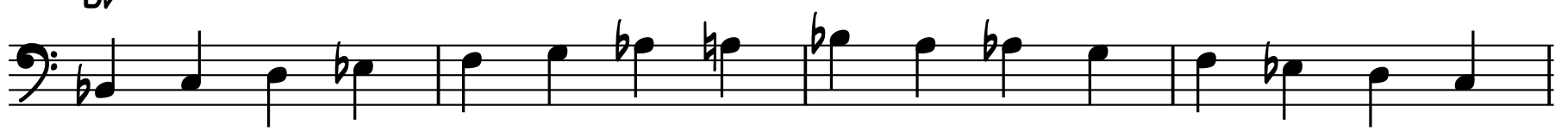

7:

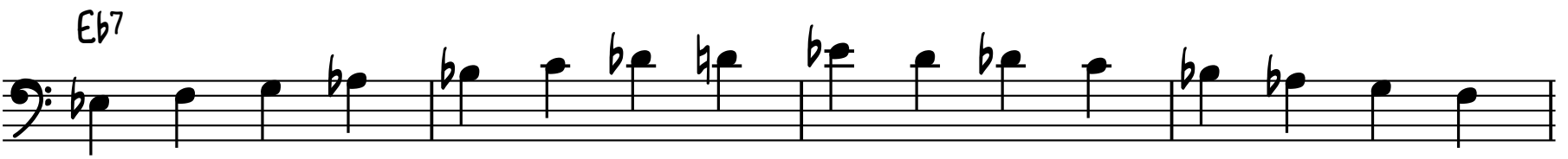

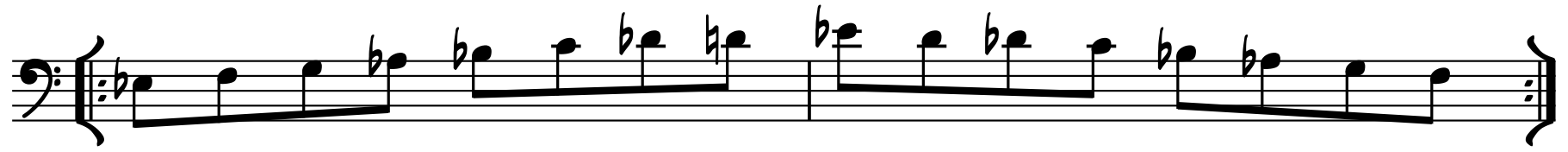

Ab7

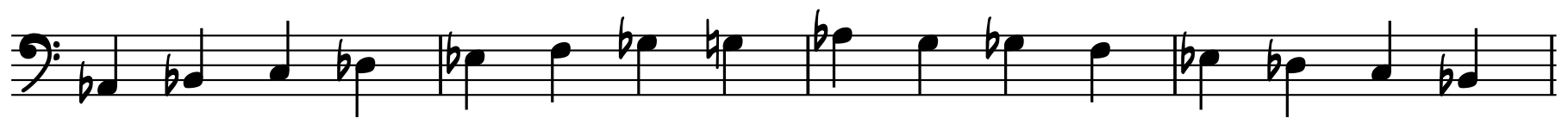

I: 
D67

\%:be be be be be be he be e be be be be pe be

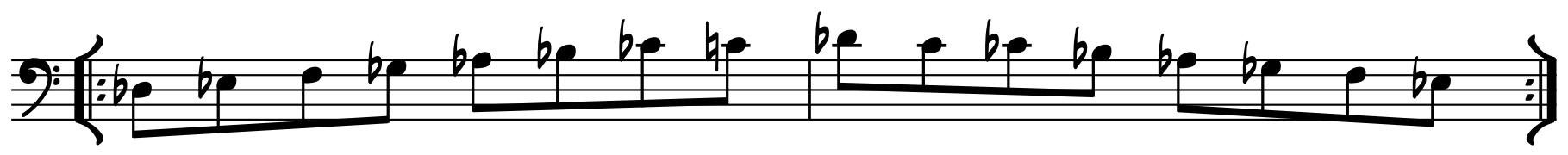

$G 67$

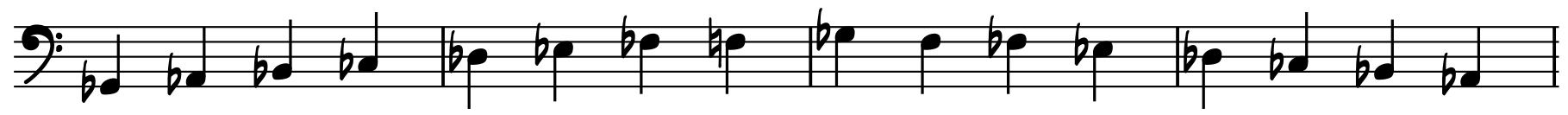

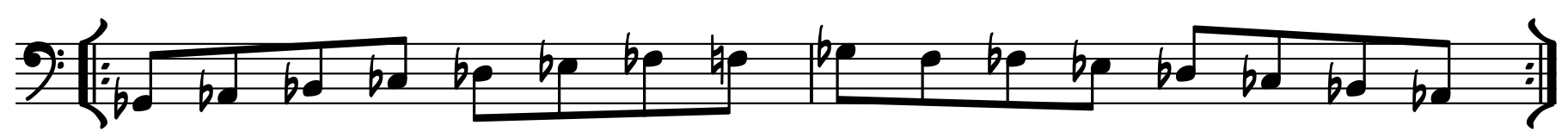

B7

9.

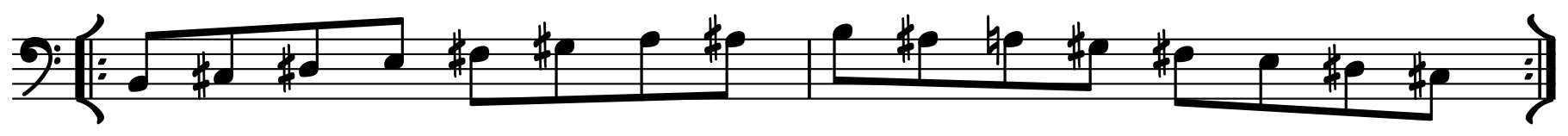

E7

2.

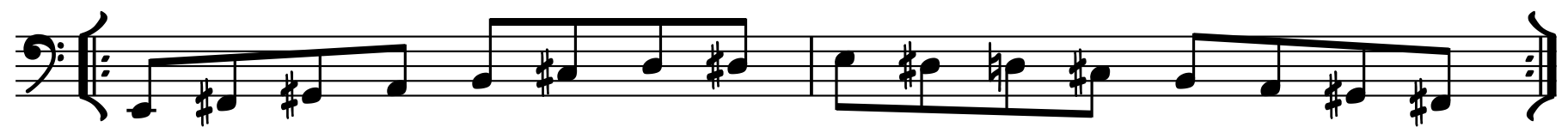

A7

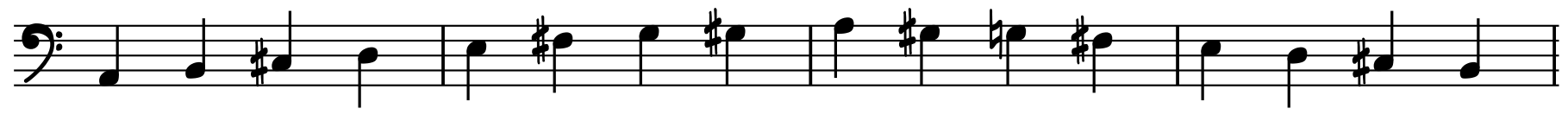

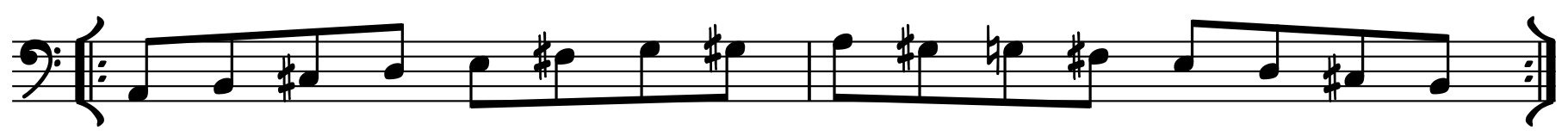



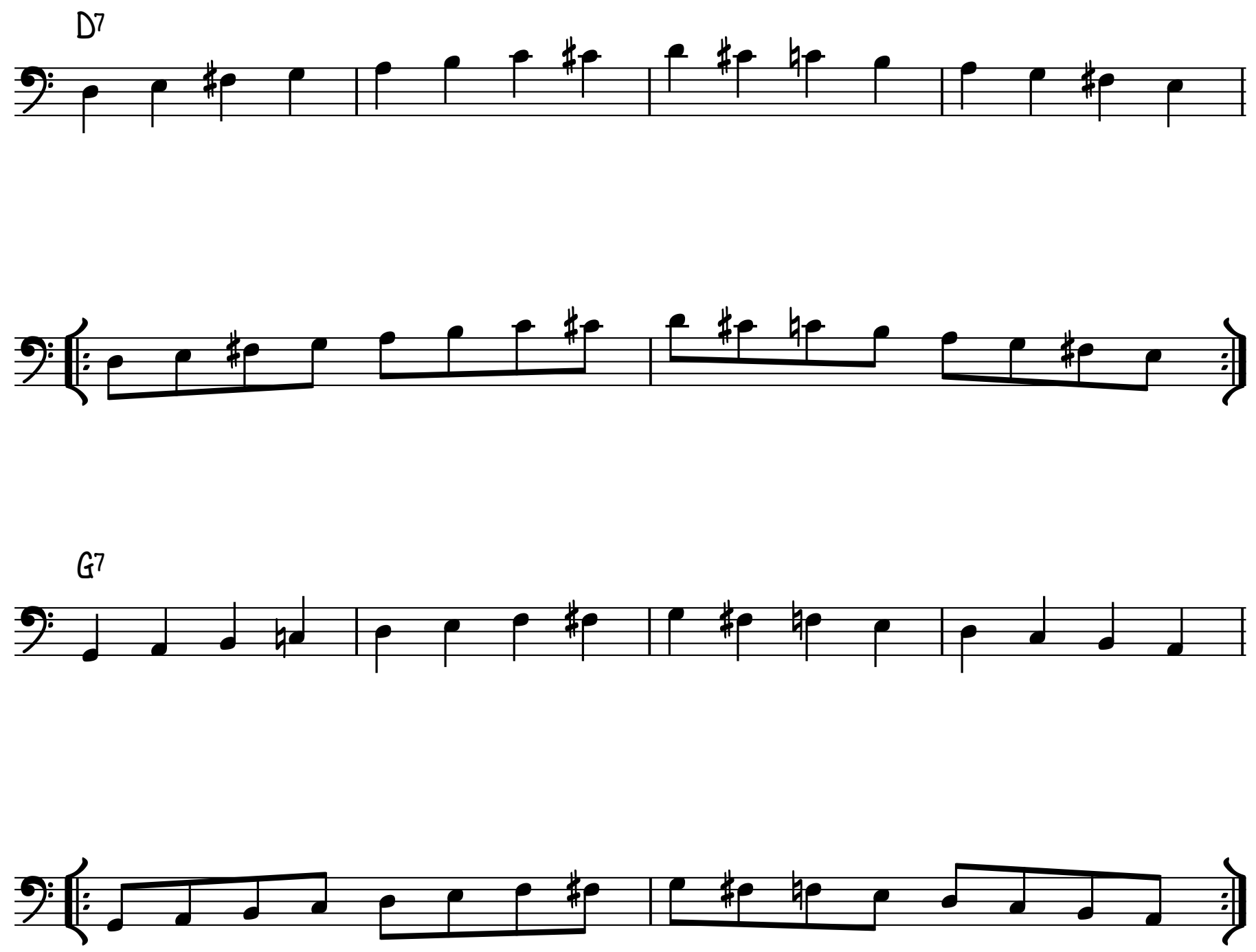

ONCE YOU HAVE MASTERED THIS AT THE FASTEST TEMPO, TRY TO PRACTICE THESE SCALES STARTING ON THE 3RD, 5TH AND FLAT 7TH SCALE DEGREES.

C7

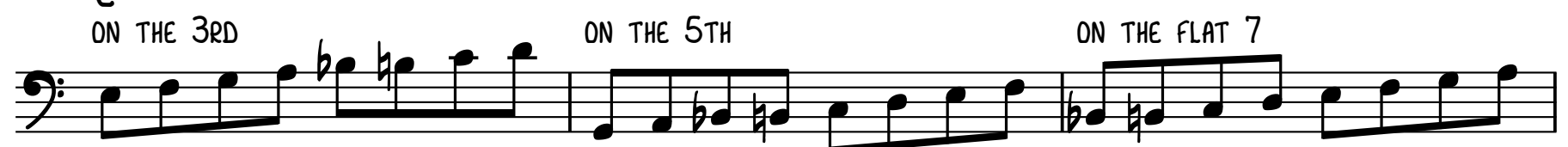




\section{SCALE EXERCISE 3 - MINOR BEBOP SCALES}

TRACK $19-112$ BPM TRACK $20-132 \mathrm{BPM}$

$0^{D_{m} 7}$
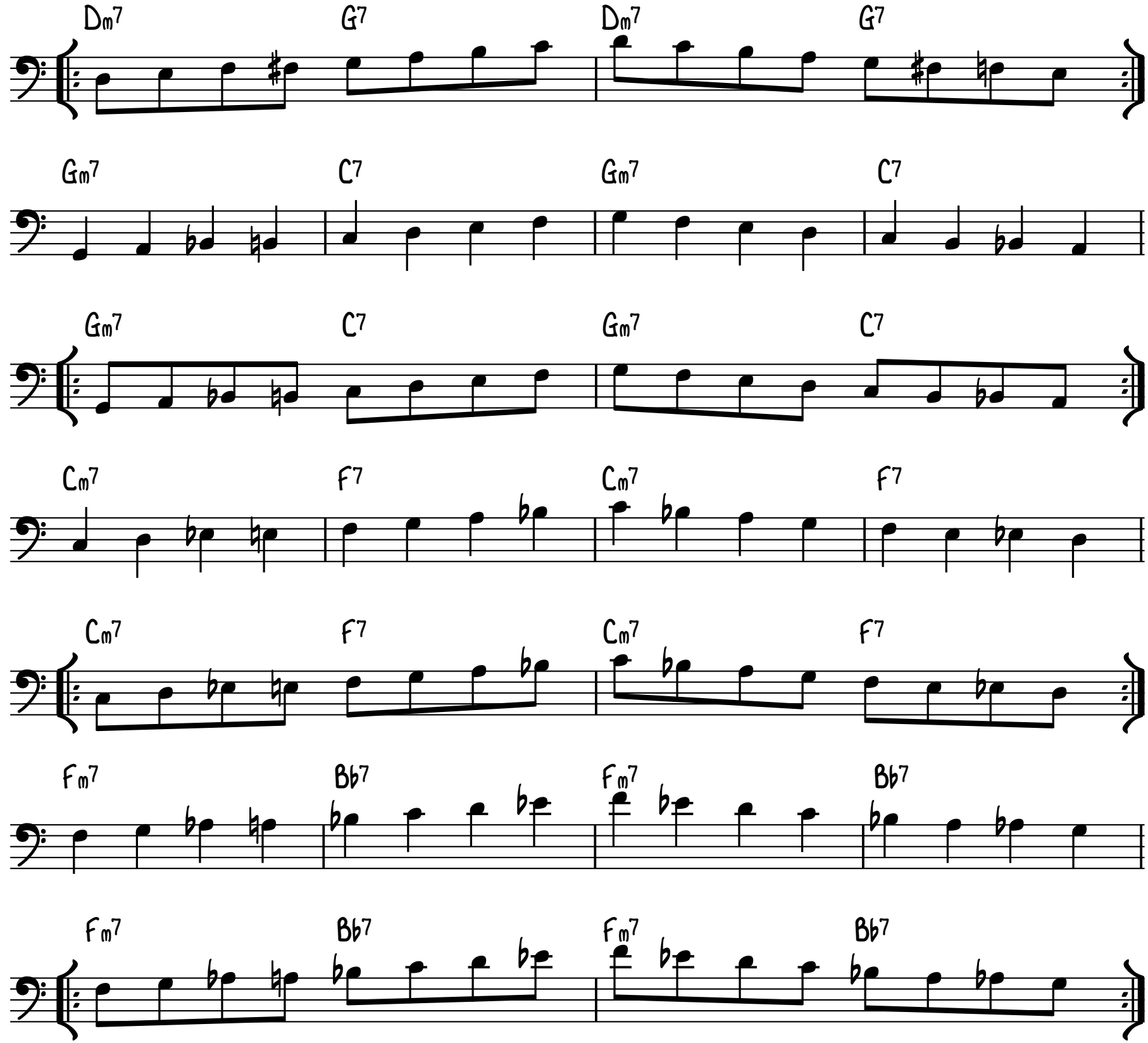
Fob g(c) $\begin{array}{llll}E b m^{7} & A b 7 & E b_{m} 7 & A b 7\end{array}$

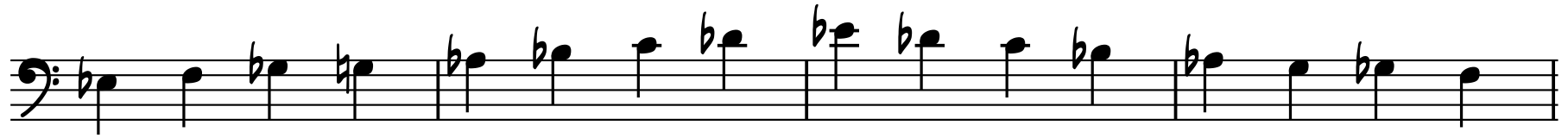

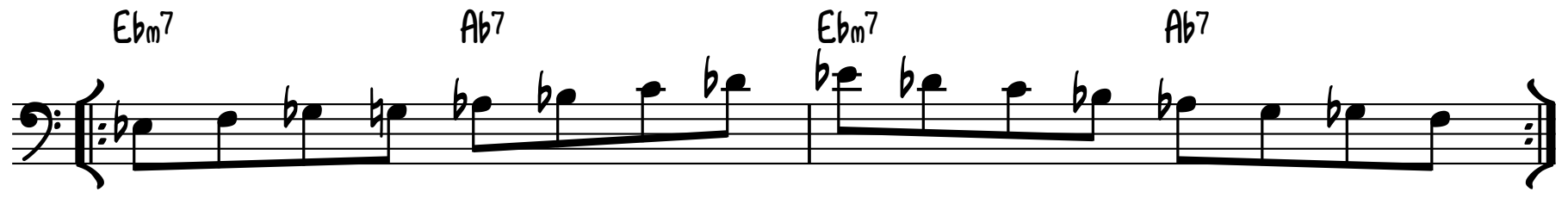
$\mathrm{Abm}^{7}$
D67
$\mathrm{Abm}_{\mathrm{m}} 7$
D67

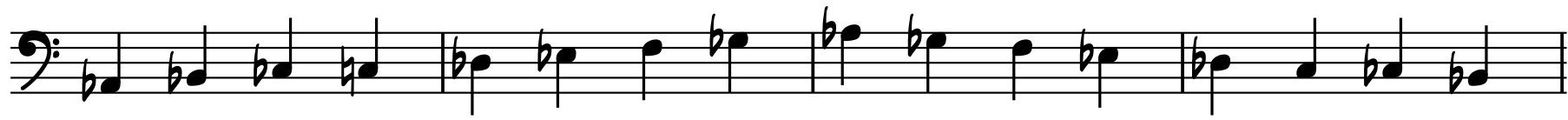

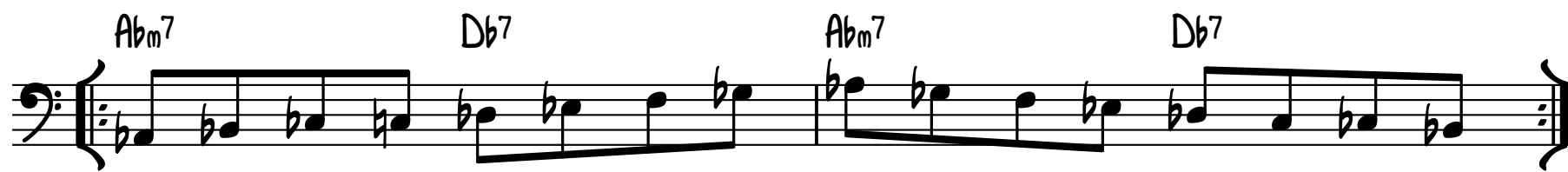
$D b_{m} 7$
Gb7
$\Delta b_{m} 7$
$G 67$

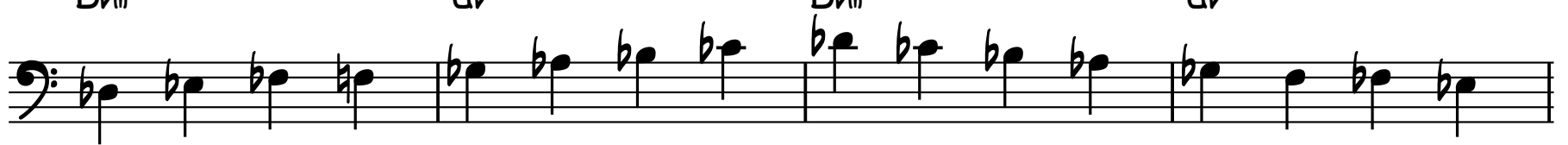
$D b_{m} 7$
Gb7
$\Delta b_{m} 7$
$G b^{7}$

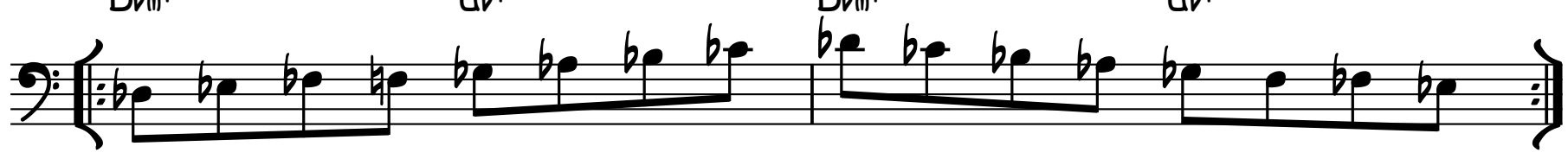
$\mathrm{F} \#_{m} 7$
B7
$F \sharp_{m} 7$

B7

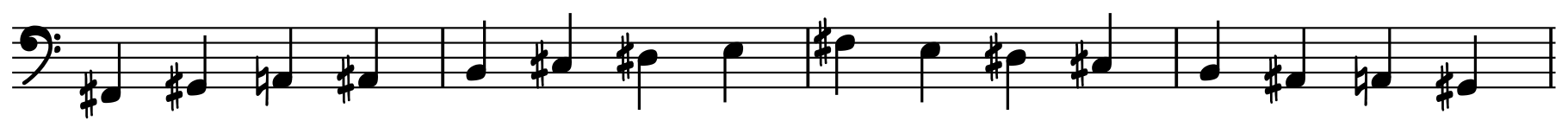

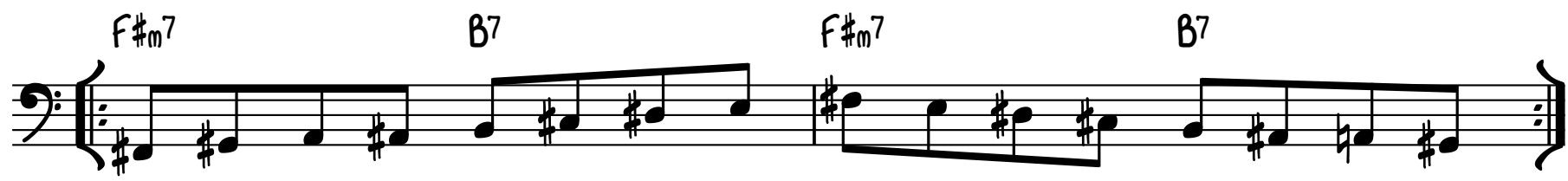



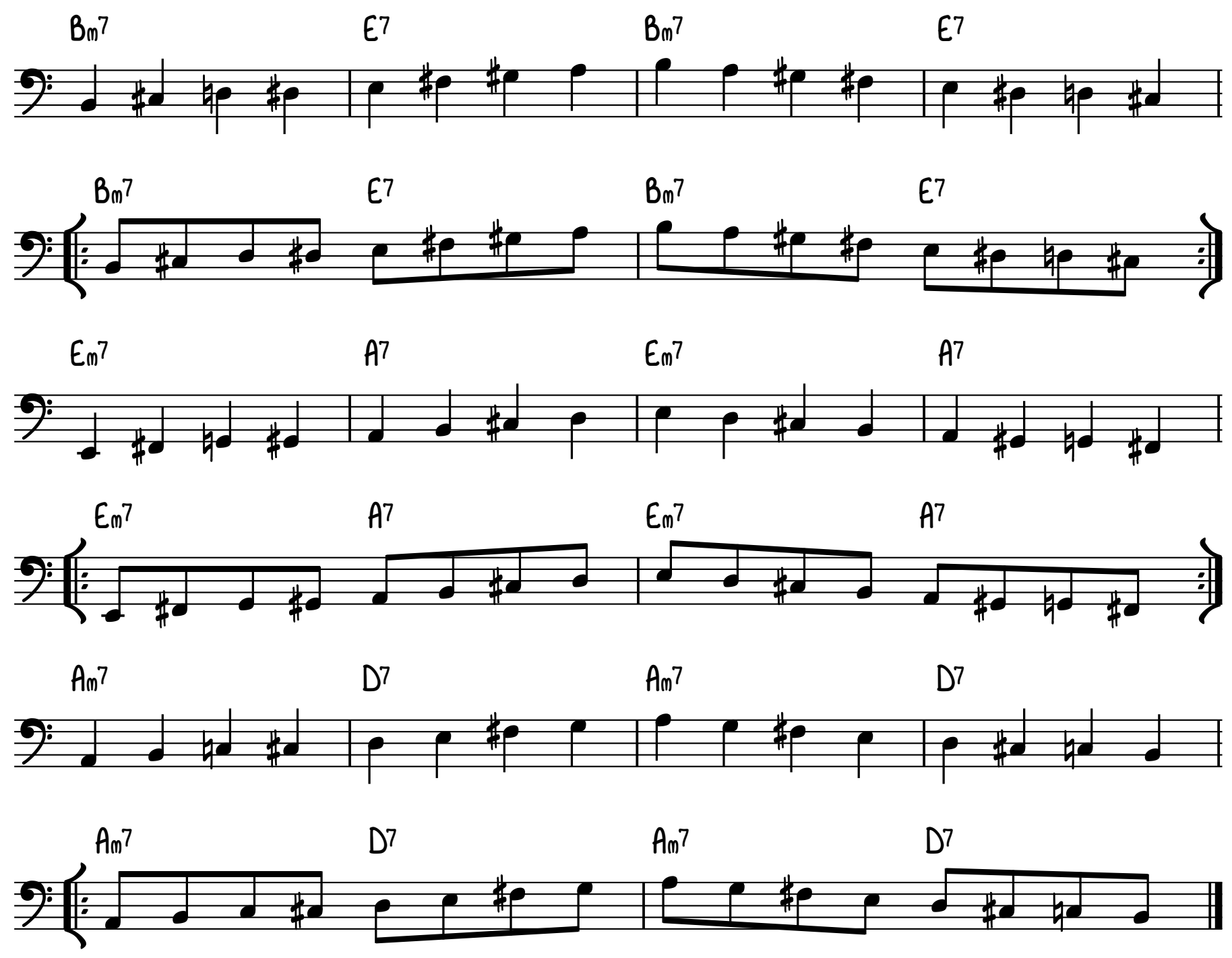

THIS IS THE 5TH MODE OF THE DOMINANT BEBOP SCALE. FOR FURTHER EXPLORATION, REFER TO THE EXPANSION IN SCALE EXERCISE 2. 
TRACK 22 - 100 BPM

$\mathrm{Gm}^{\text {SWING }} \mathrm{C7} \quad \mathrm{FmAj7} \quad \mathrm{Fm}^{7} \quad$ Bb7 EbMaj7 TRACK 24-140 BPM -
$E b_{m} 7$
Dbmaj7
$\mathrm{C}_{m} 7$
$F \$ 7$
BMAj7

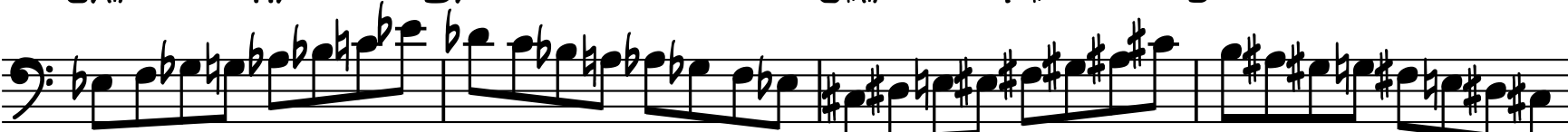
$\mathrm{Bm}_{\mathrm{m}}$
E7
Amaj7
$\mathrm{Am} 7$
D7
$G^{\text {maj7 }}$

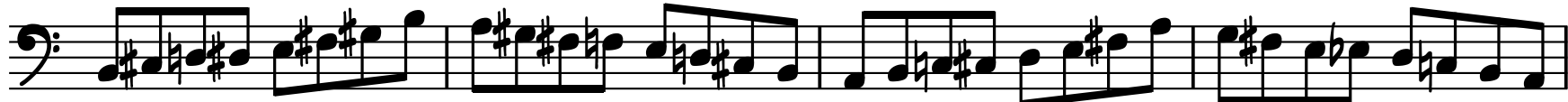
$B b_{m} 7$
$E 67$
Abmaj7
$\mathrm{Abm}_{\mathrm{m}} 7$
D67
Gbmaj7

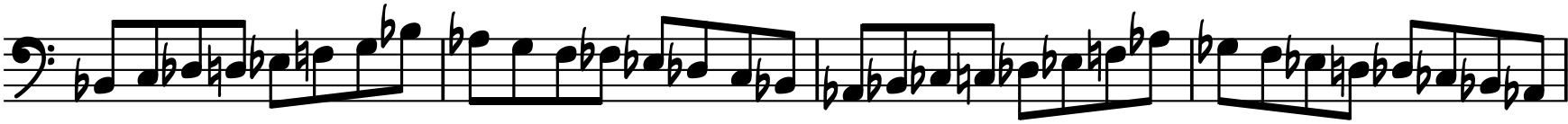

$F \#_{m} 7 \quad B 7 \quad E^{m a j 7} \quad E_{m} 7 \quad A^{7} \quad D^{m A j 7}$

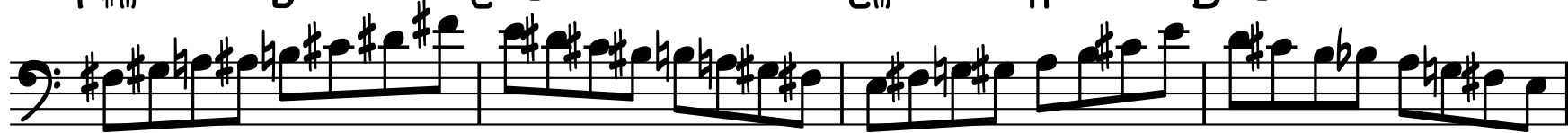

$\mathrm{Dm}^{7}$

G7

CMAj7

$\mathrm{Cm}^{7}$

F7

Bbmaj7

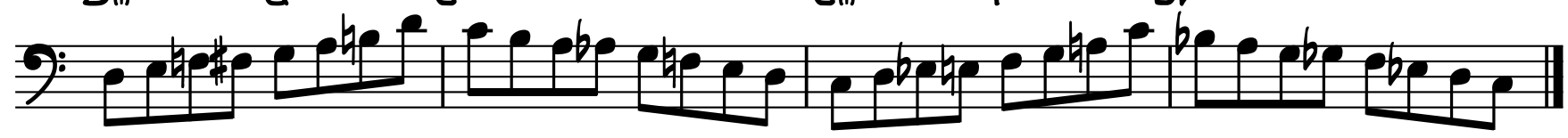




\section{ACOUSTIC BASS \\ TWO BAR II-V}

TRACK $25-100$ BPM

TRACK $26-120 \mathrm{BPM}$

$\mathrm{Am}^{7}$

D7

GMAj7

TRACK 27 - 140 BPM

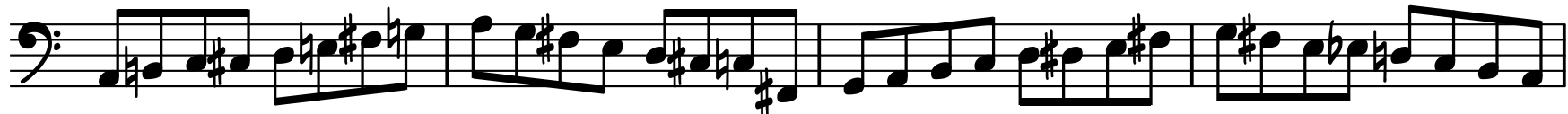
$\mathrm{Gm}^{7}$
C7
Fmaj7

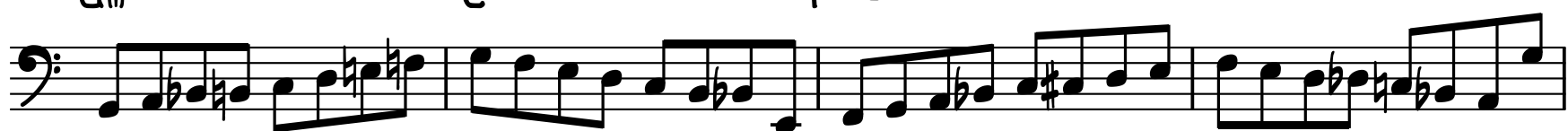

$\mathrm{Fm}_{\mathrm{m}} \mathrm{F}$

Bb7

Ebmaj7

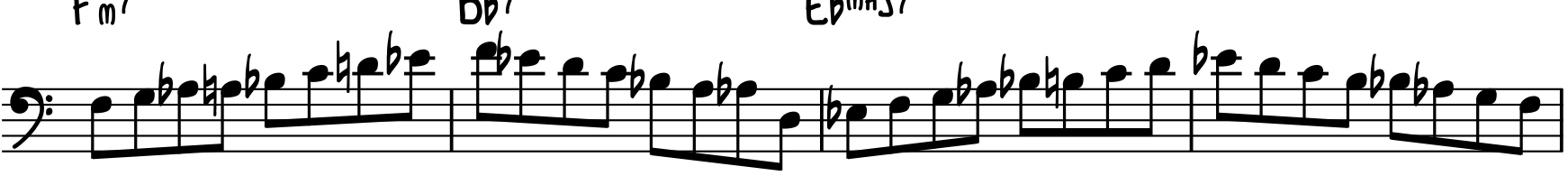

$E b_{m} 7$

Ab7

Dbmaj7

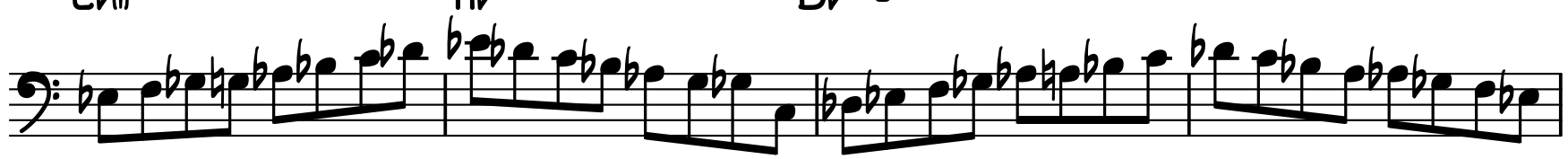

2\#年

9:

9\#湔

9: $B_{m}{ }^{E 7} A^{\mathrm{mij}}$

$\mathrm{Cm}^{7} \quad \mathrm{F7} \quad$ Bbmaj7

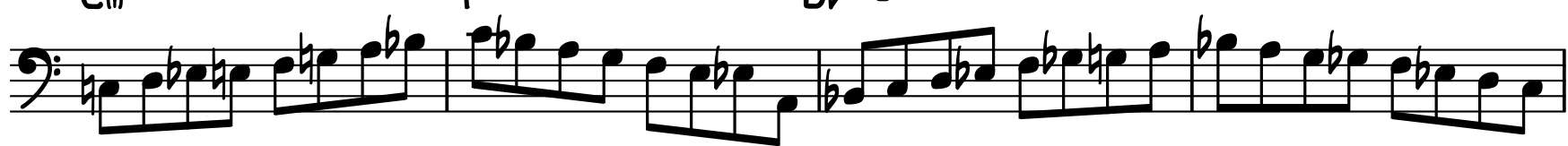


ACOUSTIC BASS

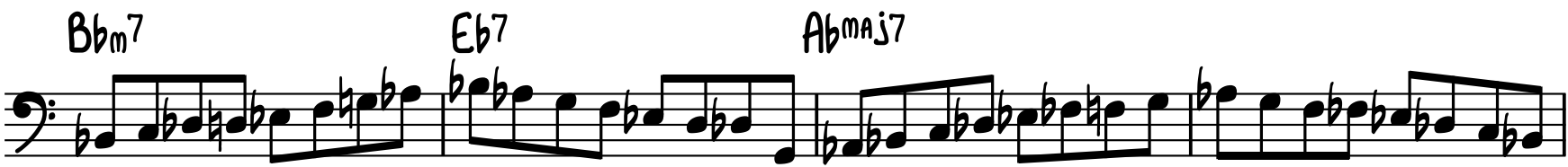

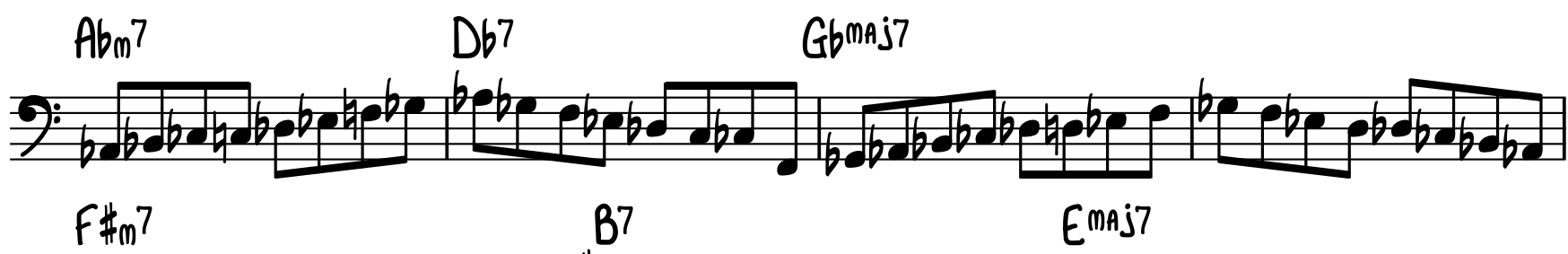

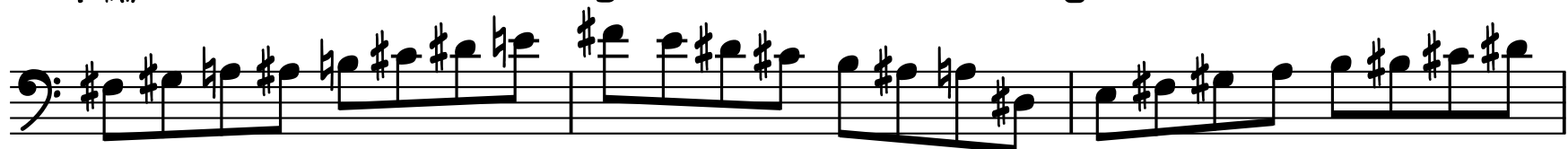

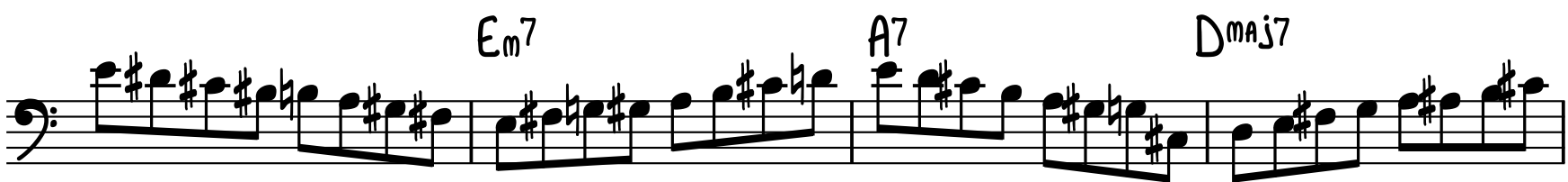

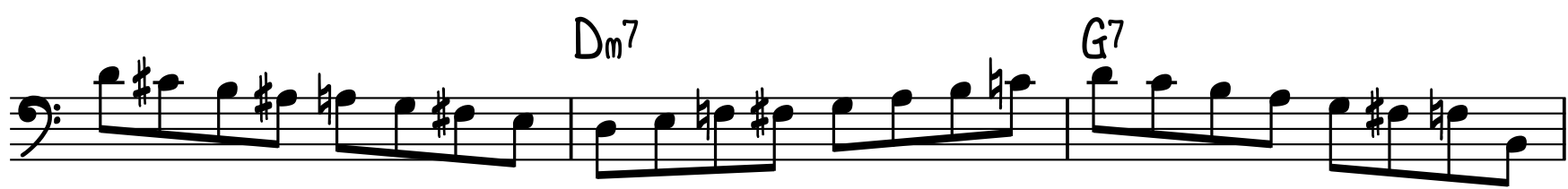

Cmaj7

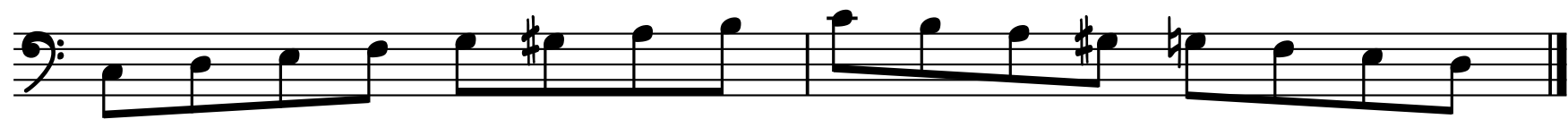




\section{ACOUSTIC BASS \\ I TO IV MODULATION THROUGH II-V}

TRACK 28 - $92 \mathrm{BPM}$

TRACK 29 - 112 BPM

TRACK 30 - 132 BPM
Fmaj7
$\mathrm{Cm}^{7}$
F7
Bbmaj7
$\mathrm{Fm}^{7}$
B67

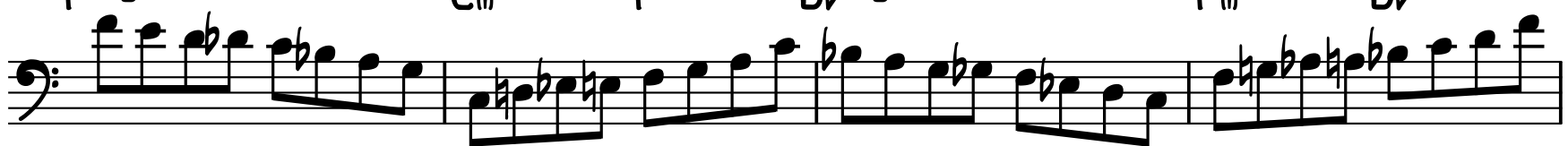
Ebmaj7
$\mathrm{Bbm} 7$
$E 67$
Abmaj7
$E b_{m} 7$
$A b 7$

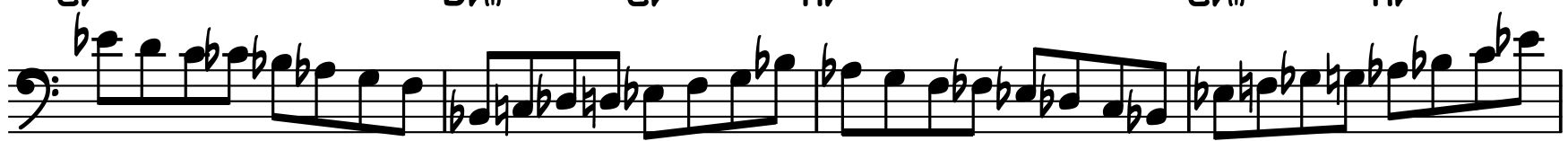
Dbmaj7
$A b m 7$
$D 67$
Gbmaj7
$C \#^{7}$
$f \sharp 7$

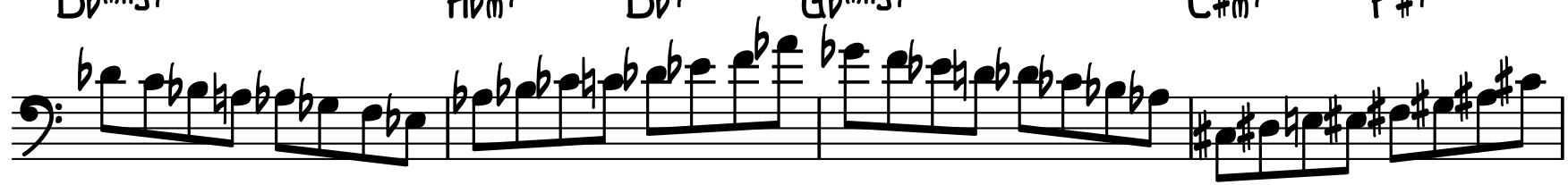

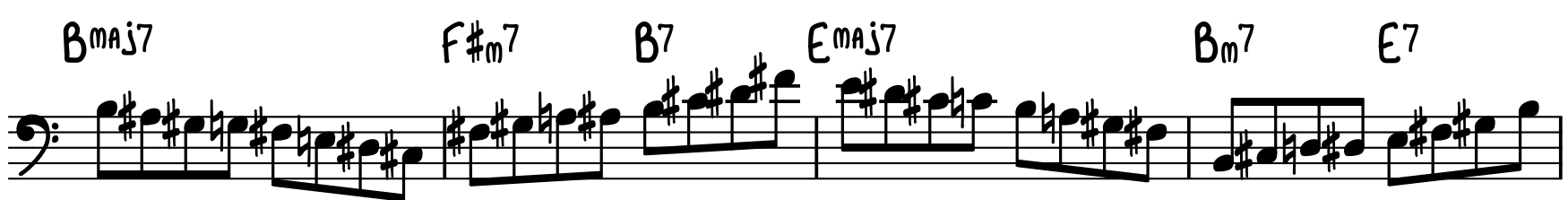

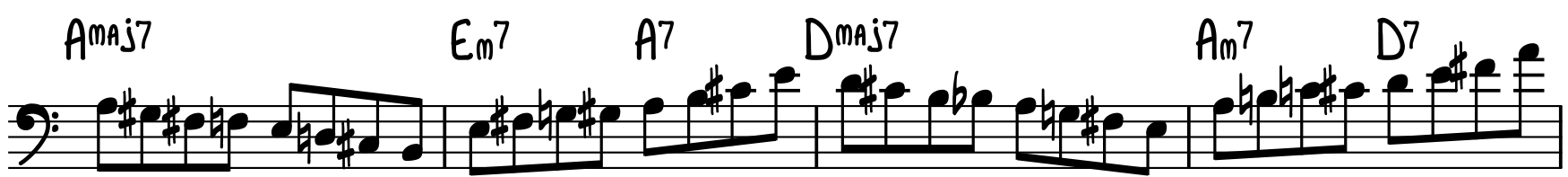
Gmaj7
$\mathrm{Dm}_{\mathrm{m}} 7$
G7
CMAj7
$\mathrm{Gm}^{7}$
C7 Fmaj7

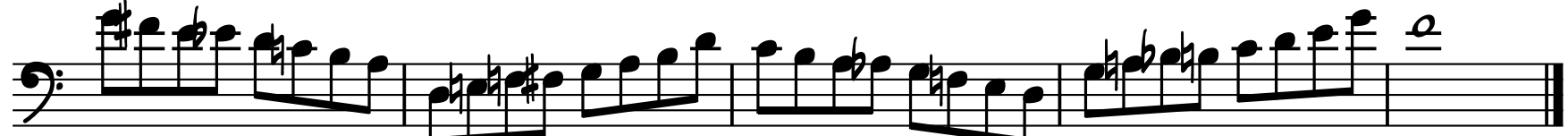


G:4 SIMILE

5 Fmaj7

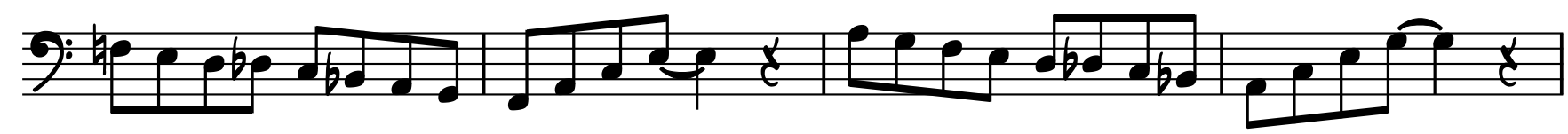

Ebmaj7
bee eb-bebe

13 Fmaj7

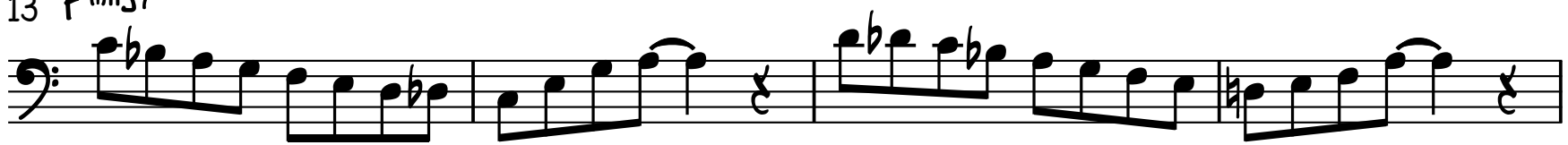

$17 G^{\text {maj7 }} \quad$ Cmaj7

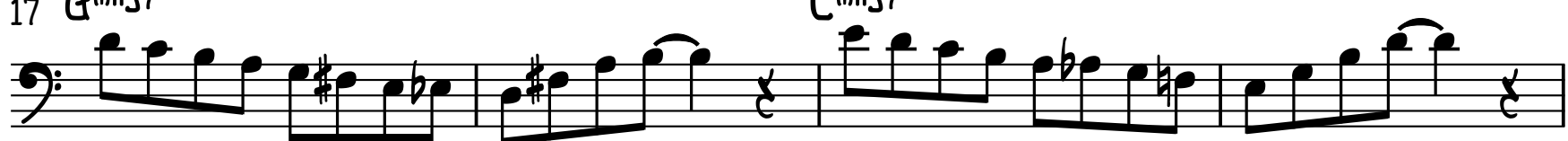

21 Fmaj7 Bbmaj7

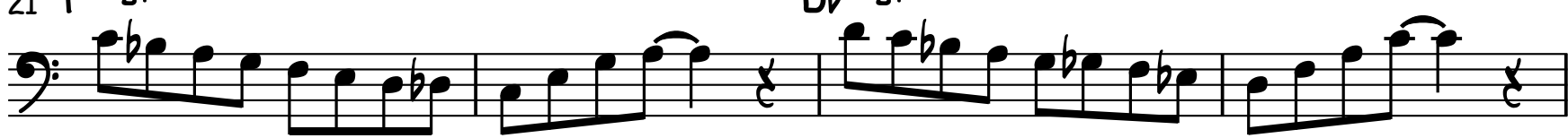

25 Ebmaj7

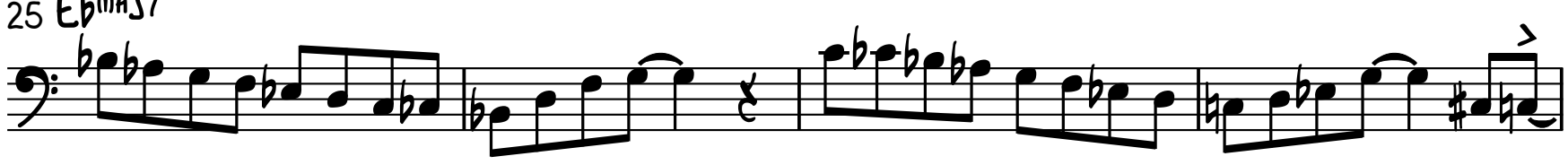

29 Fmaj7

7) 
2

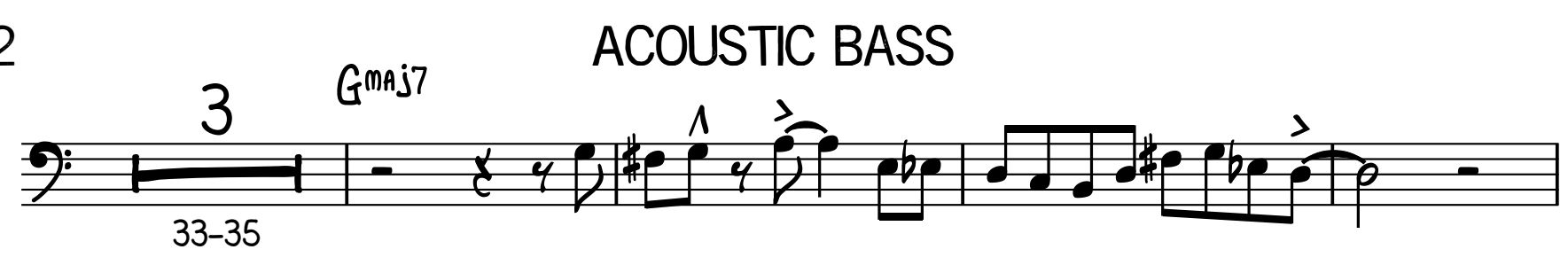

40 Fmaj7

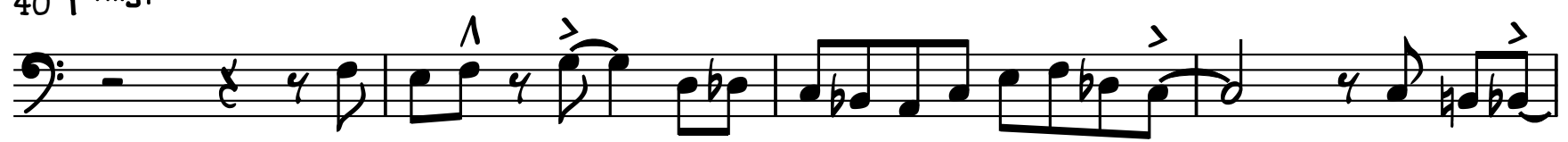

44 Ebmaj7

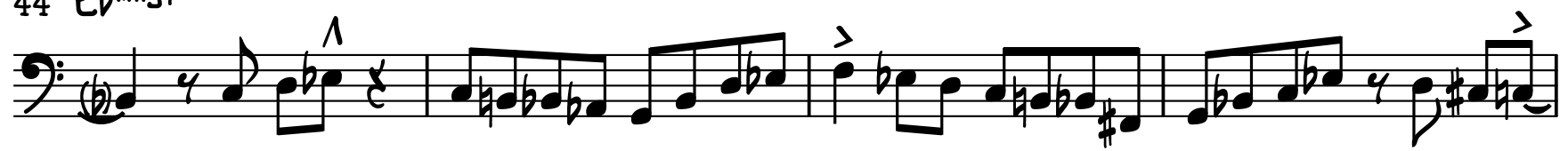

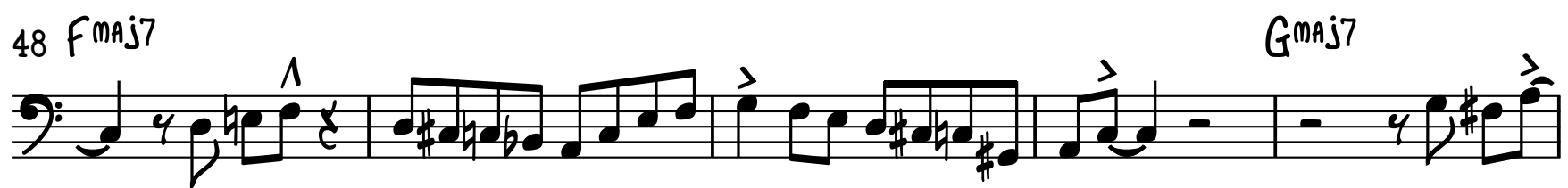

(a)

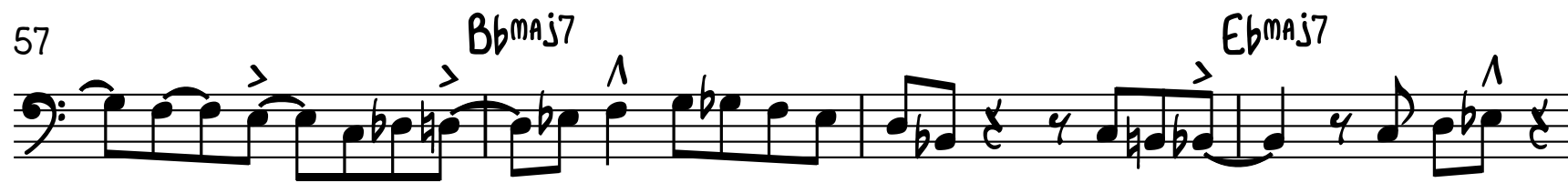

61
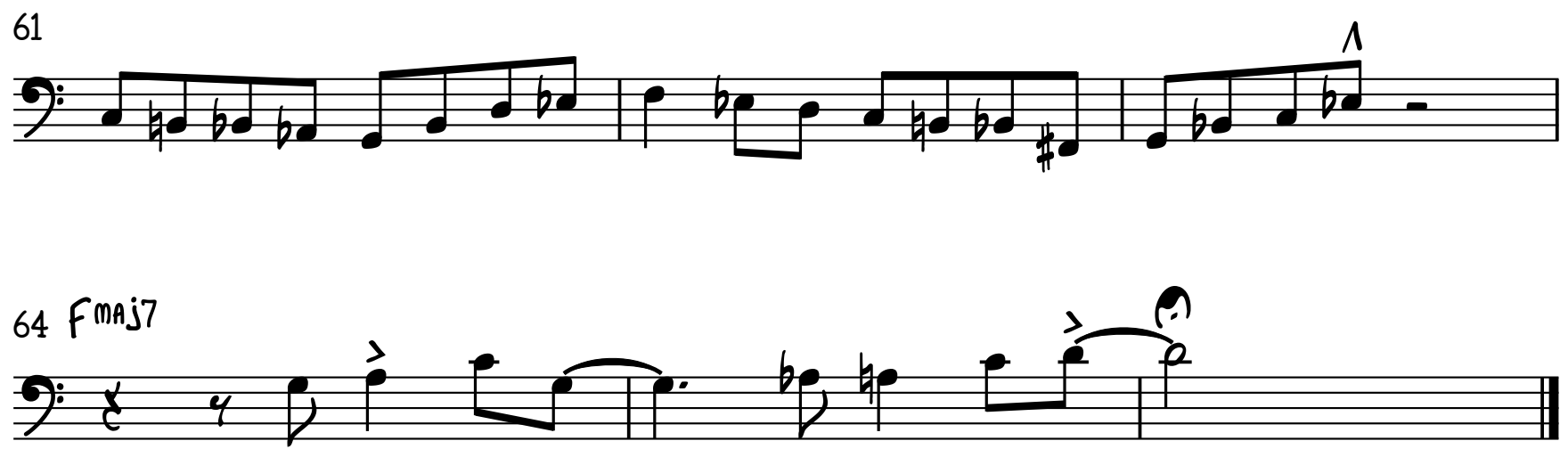
Bbmaj7

BmAj7

TRACK 9 - 152 BPM

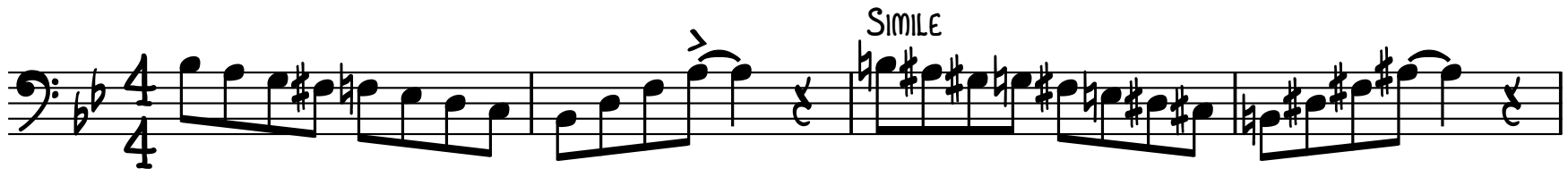

$5 \quad$ Bbmaj7

Ebmaj7

$D_{m} 7$

G7

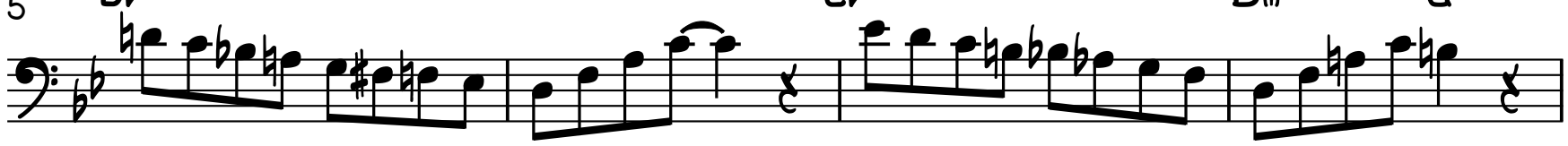

9 CMAj7

13 Cmaj7

17 Fmaj7

$21{ }^{E m^{7}}$

$21 \mathrm{Em}^{7}$

$21 \mathrm{Em}^{7}$

$21 E^{E_{m}}$

25 Cmaj7

29 Bbmaj7 $\quad$ Dbmaj7 $\quad$ Gbmaj7 $\quad$ Bmaj7

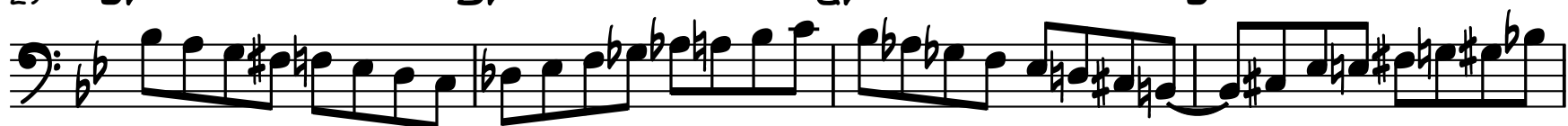


2

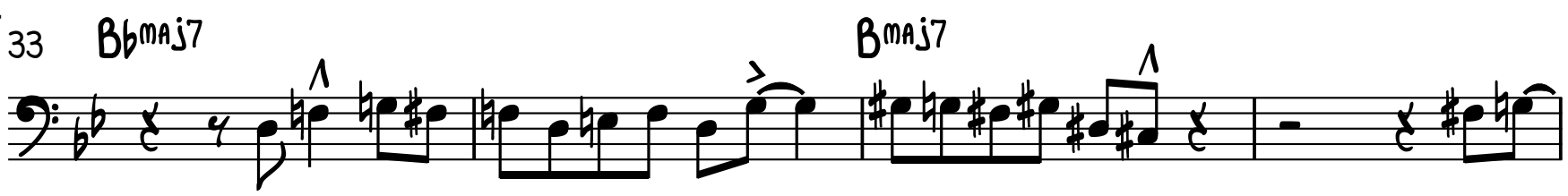

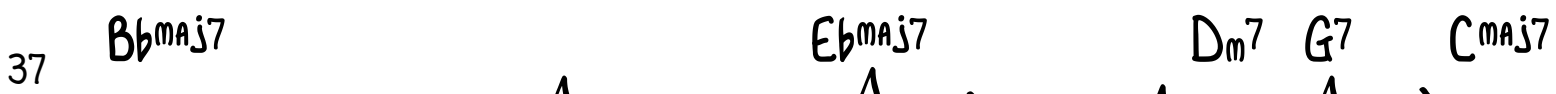

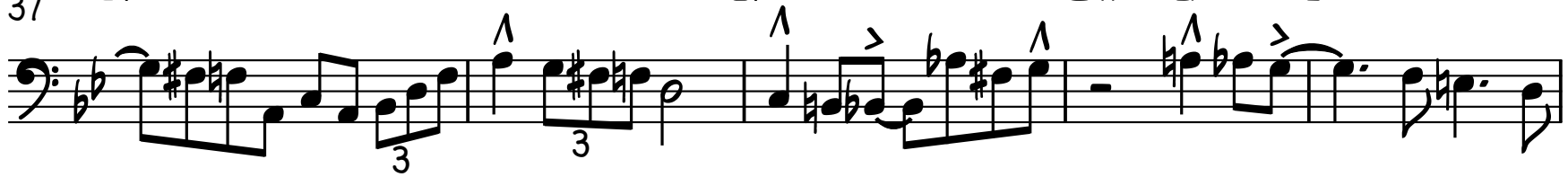

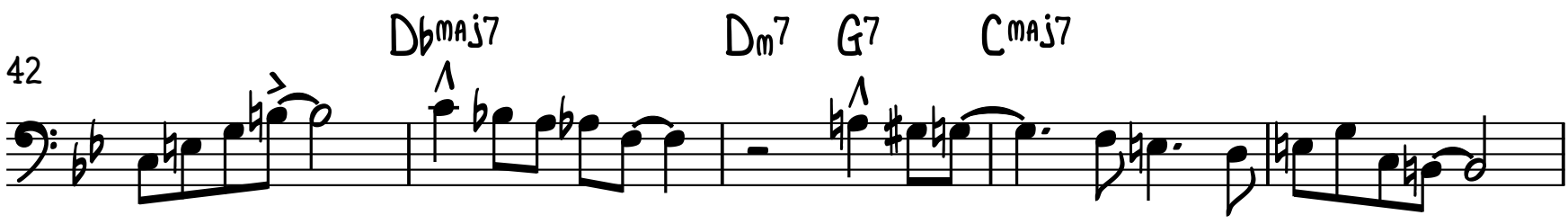

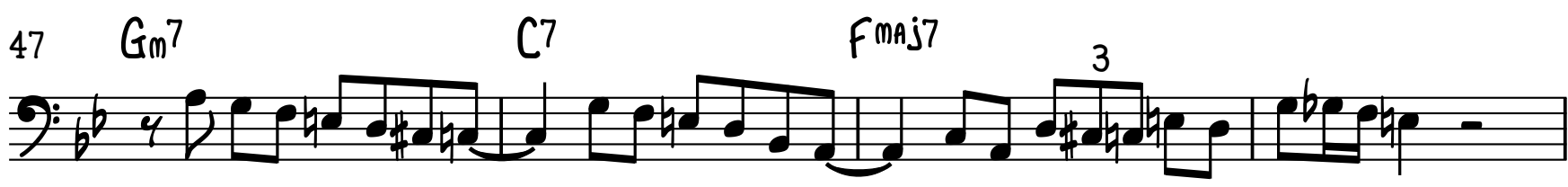

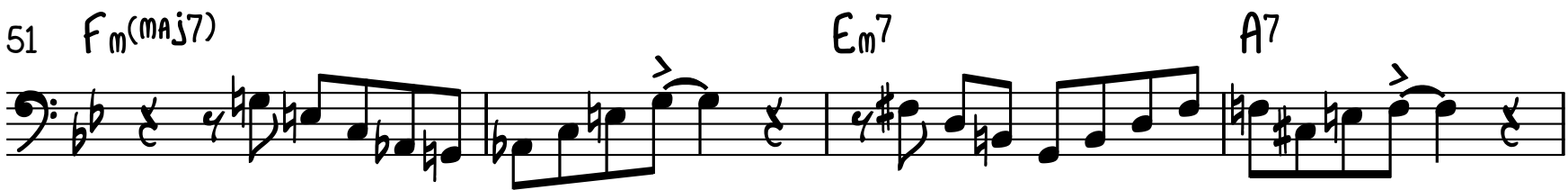

$55 \mathrm{Dm}^{7}$

59 Dhani7
62 Dbmaj7
Gbmaj7
BMAj7
Bbmaj7

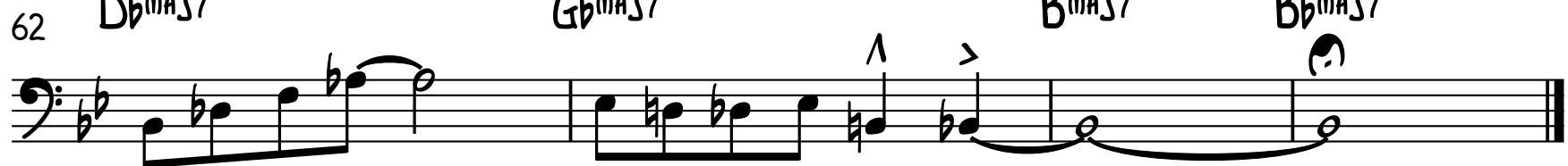


ACOUSTIC BASS

\section{ETUDE 3 - DOMINANT BEBOP}

TRACK 13 - 132 BPM

TRACK 14 - 152 BPM

(2):

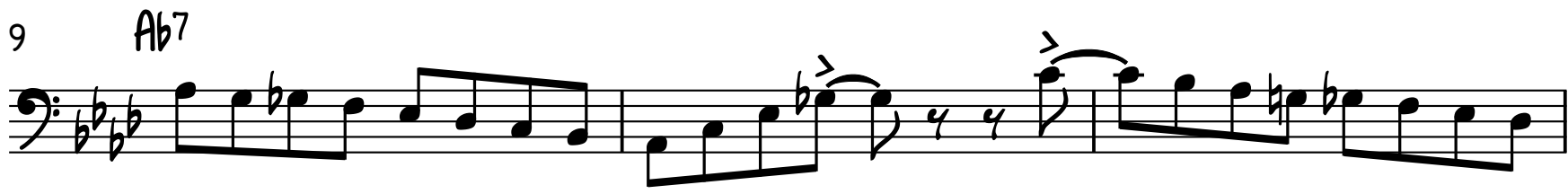

12

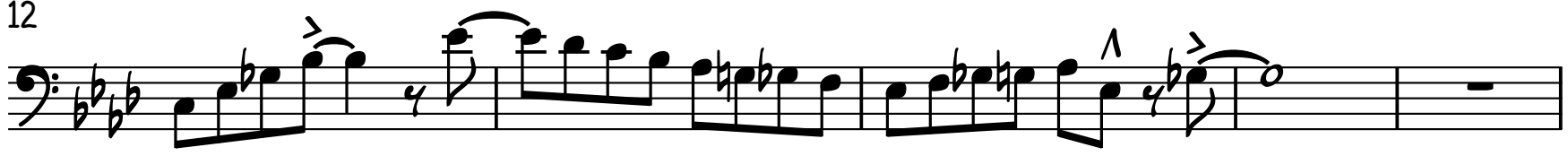

O): $b_{b}^{b} b^{67}$

$2112 b^{2}$

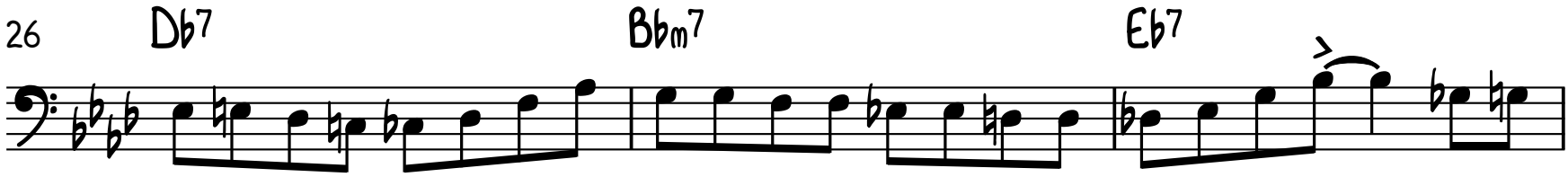

$29{ }^{a b 7} b_{b}^{b}$ 
2

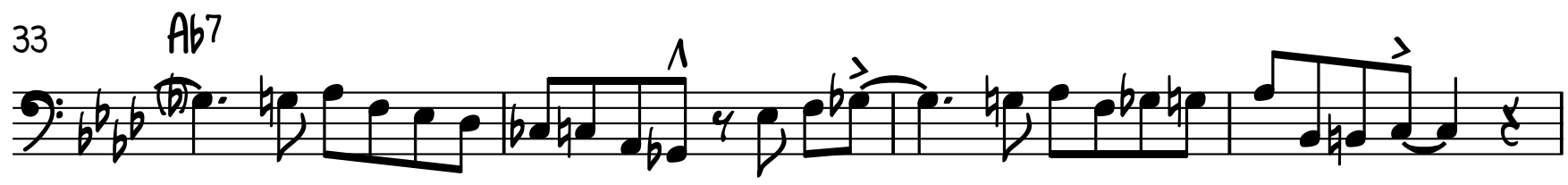

37

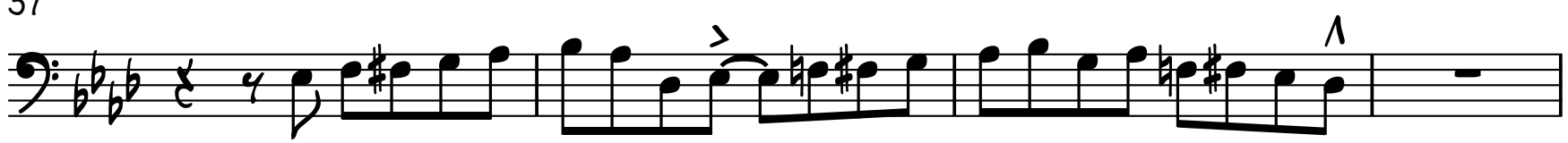

$41 \quad$ Gb7

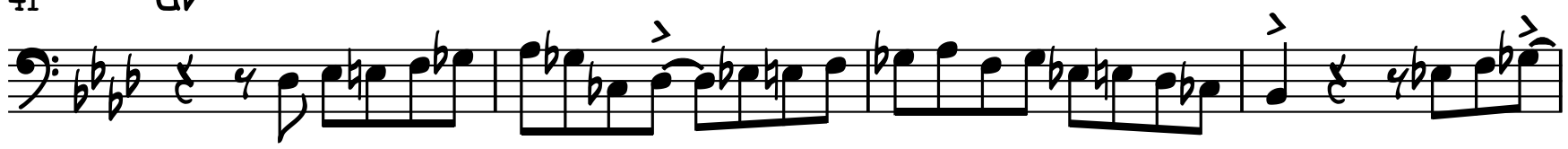

$45 \quad \mathrm{Ab} 7$

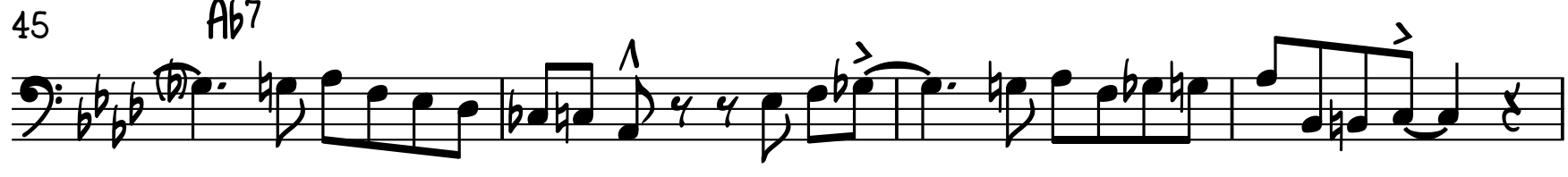

$49 \mathrm{Abm}^{2} \mathrm{Db7}$

$53 \quad A b 7$

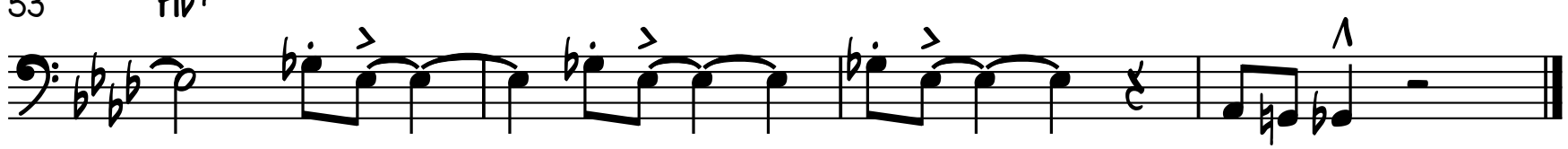




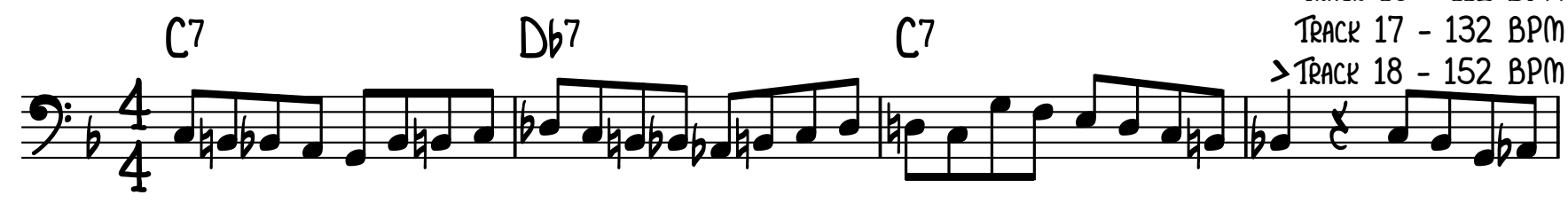

$5 \quad F 7$ Bo

9 C7 Db7

$13 \quad F 7$ Bb7 Bo

$17{ }^{A 7}$

$21 G^{7}$

$\begin{array}{llll}25 & C 7 & D 67 & C 7\end{array}$

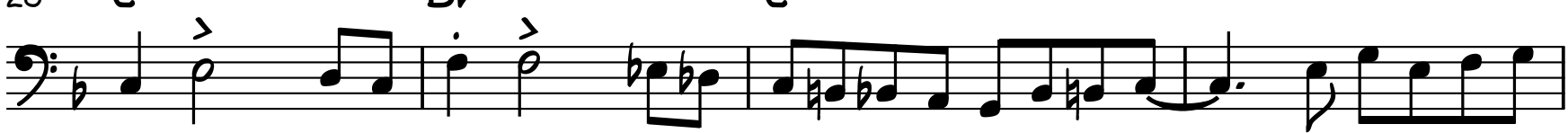

$29 \quad{ }^{F 7}{ }^{B 67}$ 
2

ACOUSTIC BASS

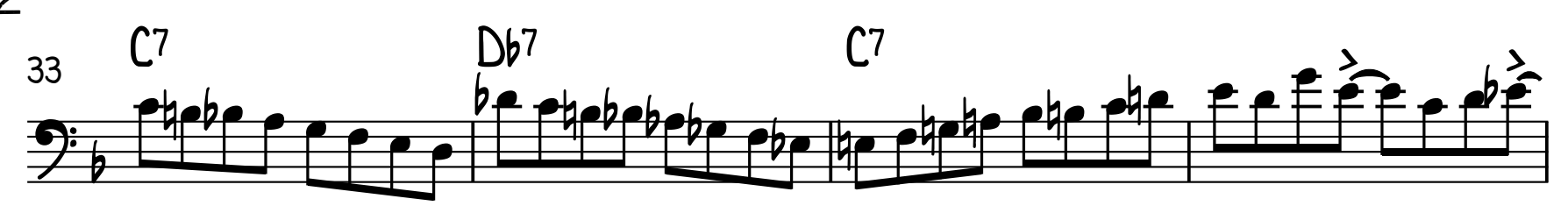

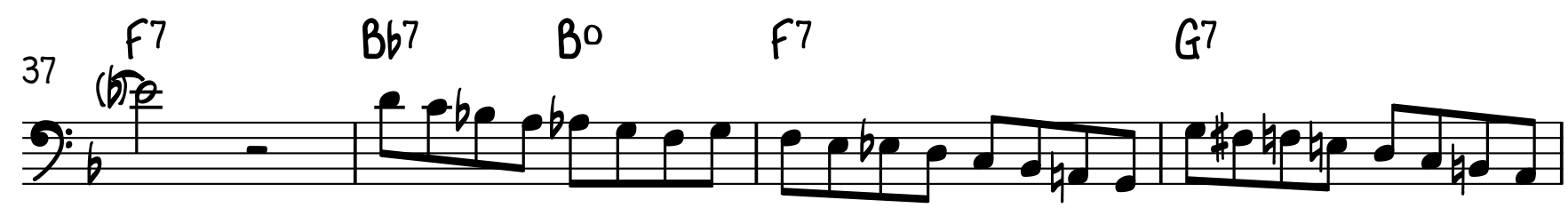

$\begin{array}{llll}41 & C 7 & D 67 & C 7\end{array}$

F: a

$\begin{array}{lllll}45 & F 7 & B b 7 & B 0 & F 7\end{array}$

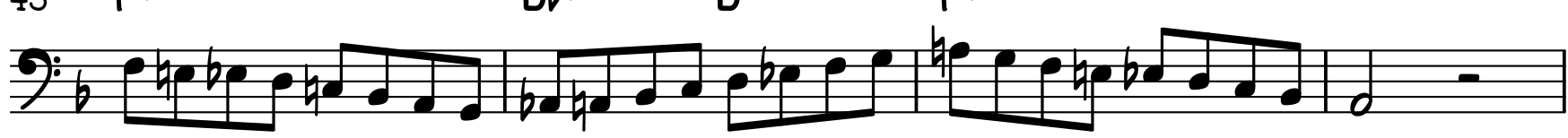

$\begin{array}{lll}49 & A 7 & D^{7}\end{array}$

Fib abe

${ }^{27} \mathrm{G}^{7}$

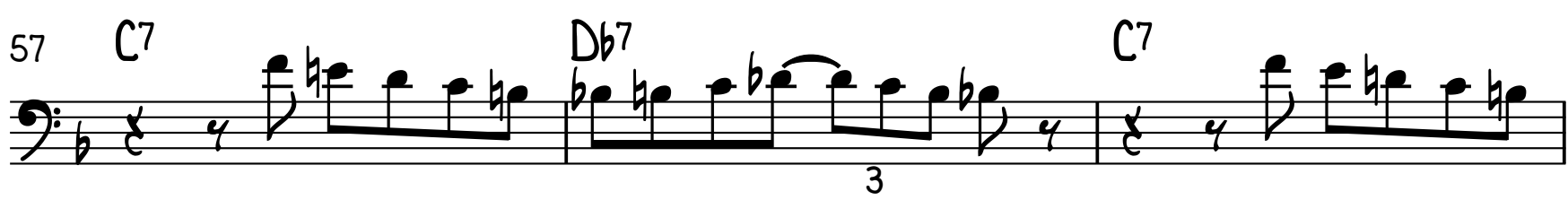

F7

Bb7 Bo

F7

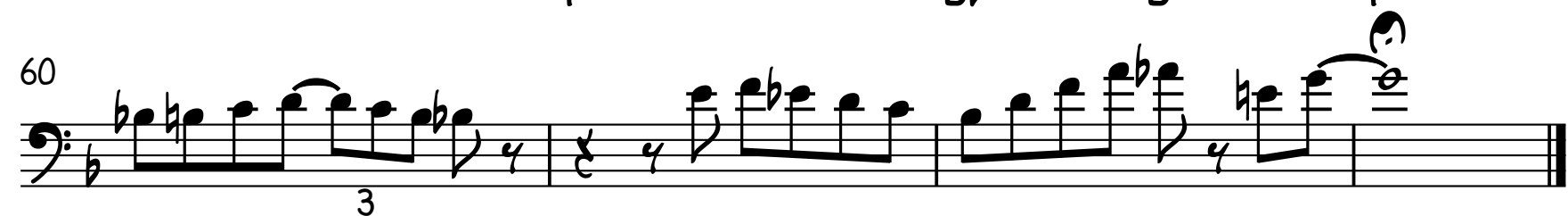


Acoostre Bass ETUDE 5 - MINOR BEBOP TRACK 32 - 120 BPM TRACK $34-140$ BPM

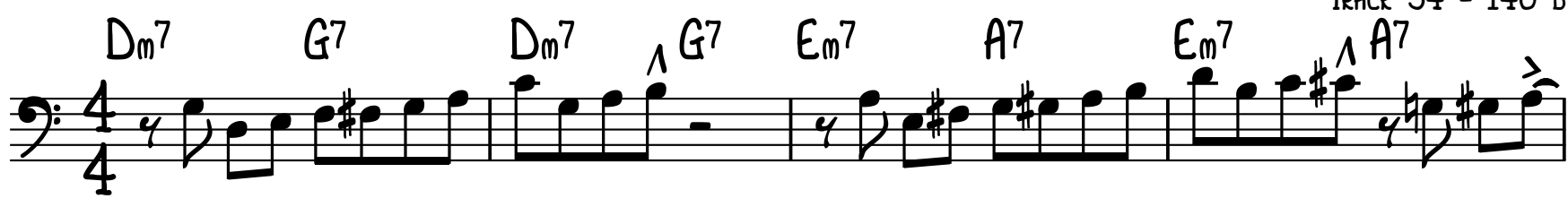
$5 \mathrm{Am}^{7} \mathrm{D}^{\mathrm{A}} \mathrm{Abm}_{\mathrm{m}} \mathrm{Db7}$ $9 D_{m} \quad G^{7} \quad D_{m} \quad G^{7} \quad E_{m} \quad$ Al $9 D_{m} \quad G^{7} \quad D_{m} \quad G^{7} \quad E_{m} \quad$ Al $9 D_{m} \quad G^{7} \quad D_{m} \quad G^{7} \quad E_{m} \quad$ Al $9 D_{m} \quad G^{7} \quad D_{m} \quad G^{7} \quad E_{m} \quad$ Al

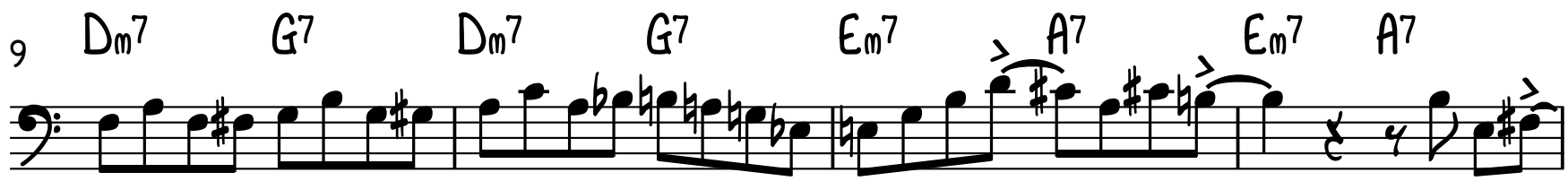

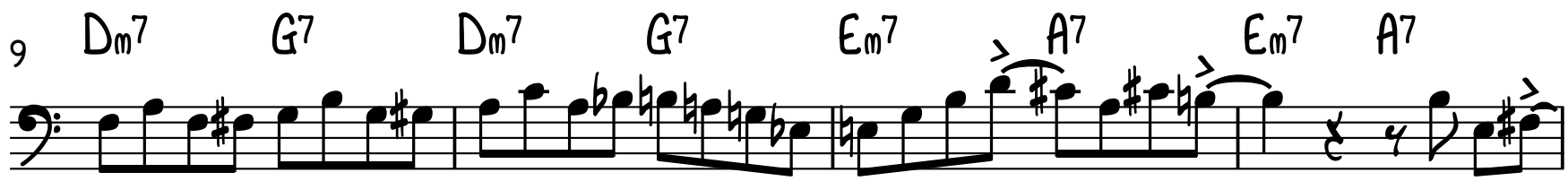

$13 \mathrm{Am}^{7} \mathrm{D7}$

$\begin{array}{llllll}17 & \mathrm{Gm}_{m} & \mathrm{C}^{7} & \mathrm{Gm}_{m} & \mathrm{C}^{7} & \text { Fmaj7 }\end{array}$ j:

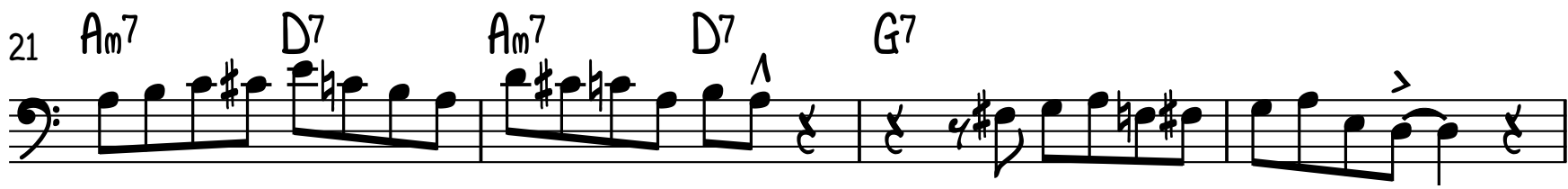

$25 D_{m} 7 \quad G 7 \quad D_{m}^{7} \quad G^{7} \quad E_{m}^{7} \quad A^{7} \quad E_{m}^{7} \quad A^{7}$

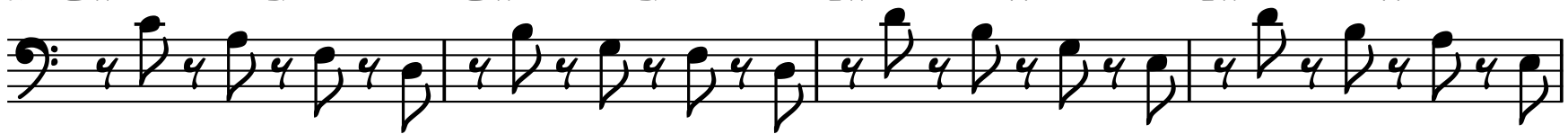

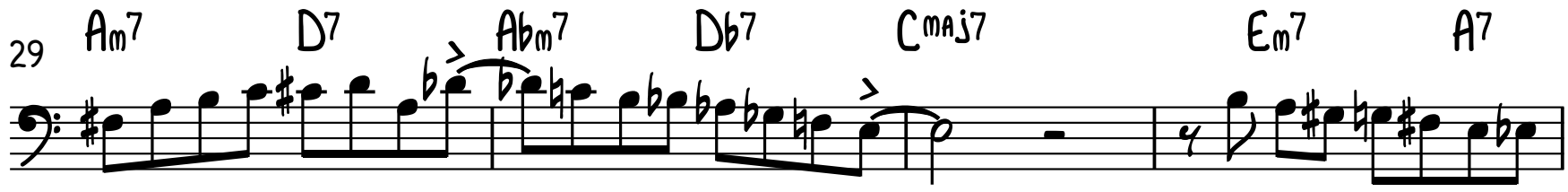

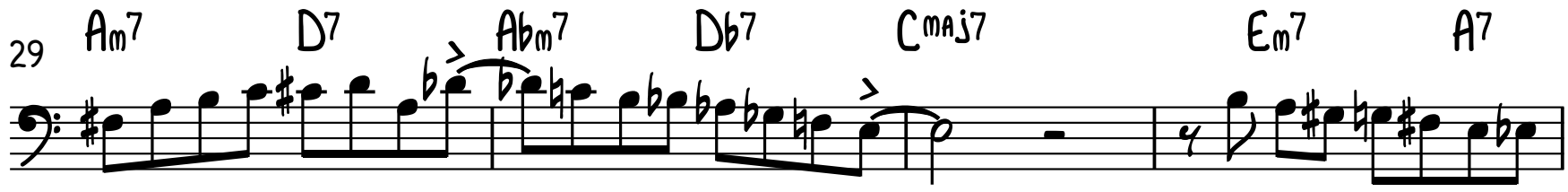

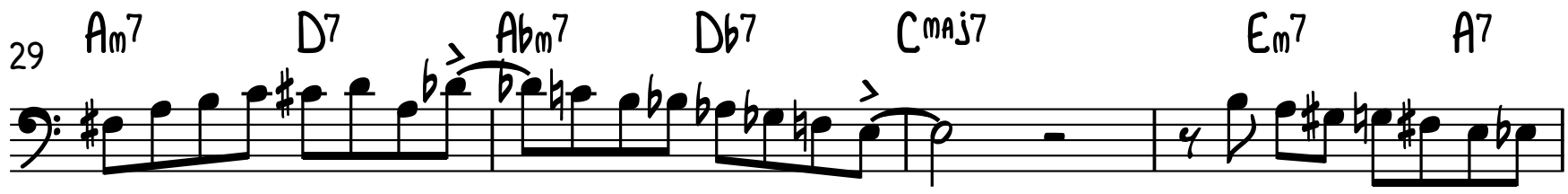

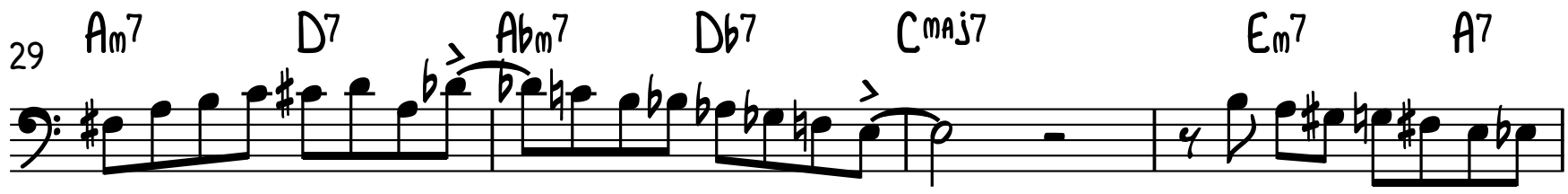

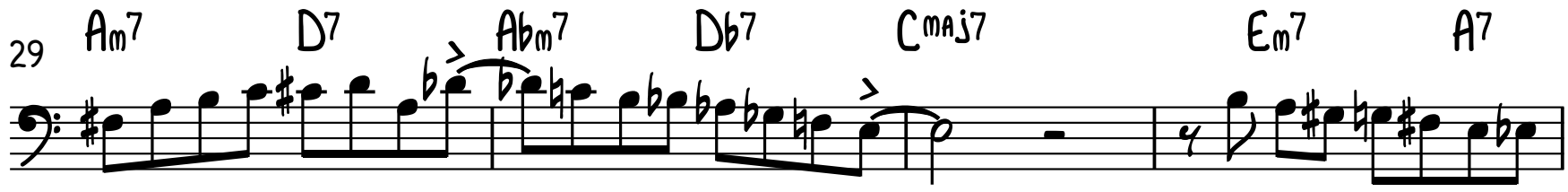

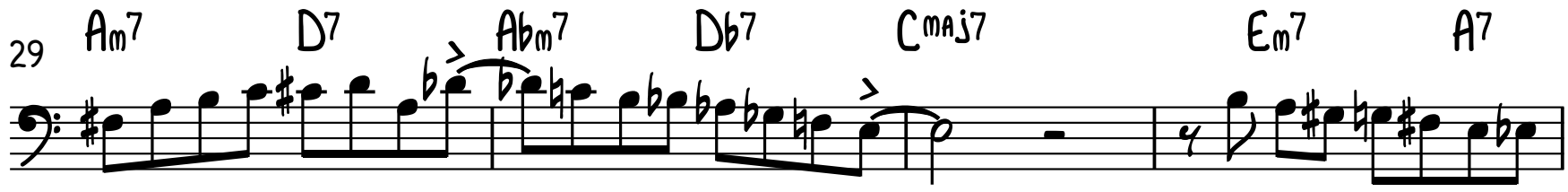


ACOUSTIC BASS

2

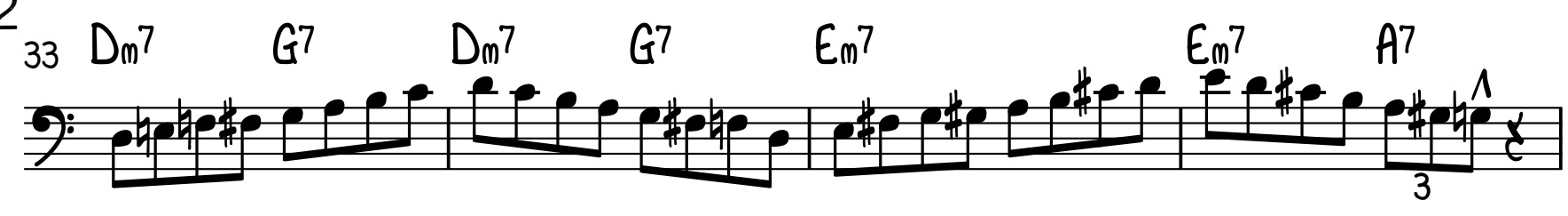
$37 \mathrm{Am}^{7}$
D7 $\quad A b m^{7}$
$D 67$
CmAj7
$E_{m} 7$
A7

9: I
${ }_{41} D_{m} 7$
$G^{7}$
$D_{m} 7$
G7
$E_{m} 7 \quad A^{7}$
$E_{m} 7$
A7

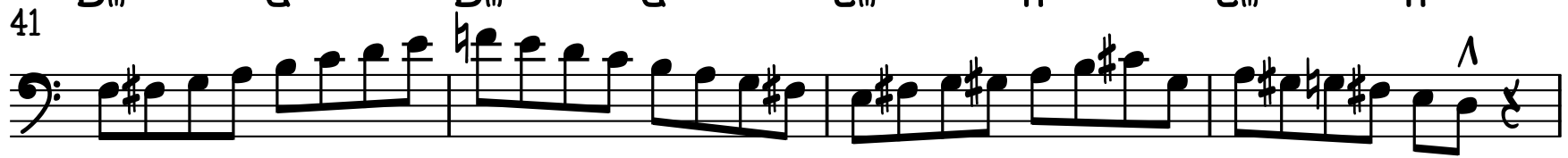

${ }_{45} \begin{array}{lllll}A m & A^{7} & A b b^{7} & D b 7 & \text { CMAj7 }\end{array}$

2:
${ }_{49} \mathrm{Gm}^{7}$
C7 $\quad \mathrm{Gm}^{7}$
C7 Fmaj7

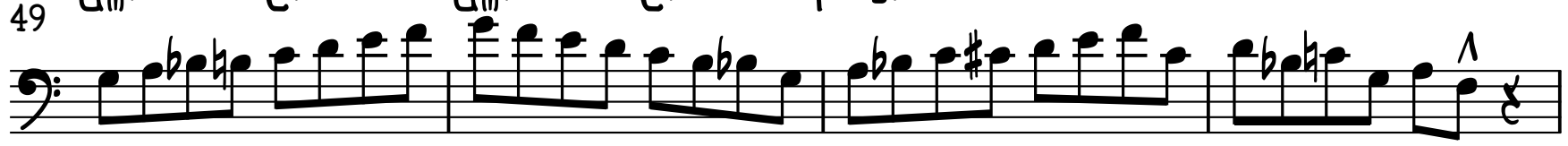

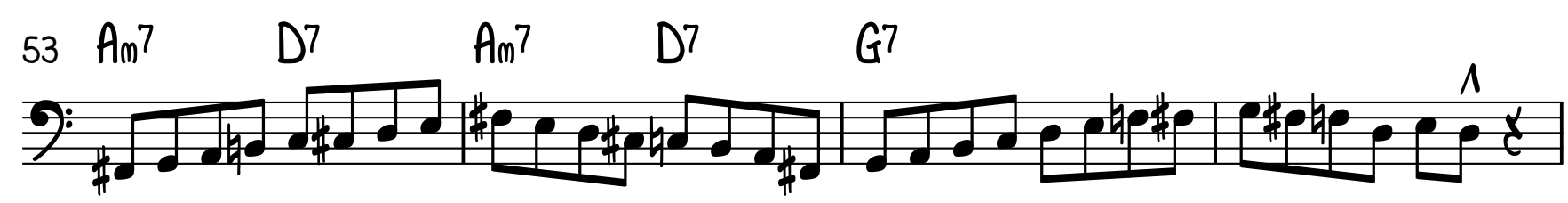

$57 \mathrm{Dm}^{7} \quad \mathrm{G}^{7} \quad \mathrm{Dm}^{7} \quad \mathrm{G}^{7} \quad \mathrm{Em}_{\mathrm{m}} \mathrm{Al}^{7}$

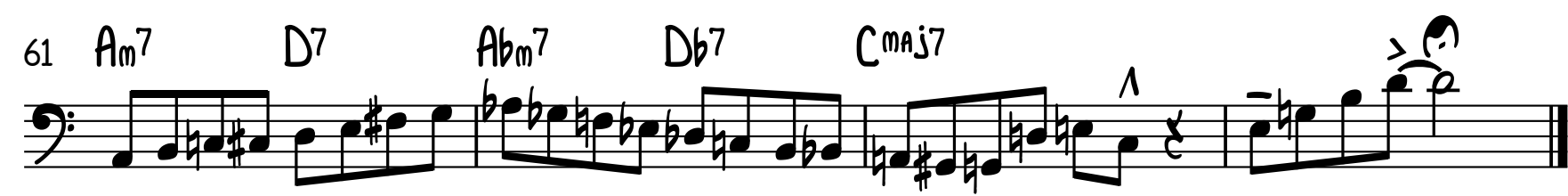

